\title{
Radiotherapy in non-small cell lung cancer : does PET make the difference?
}

Citation for published version (APA):

van Baardwijk-Renkens, A. A. W. (2008). Radiotherapy in non-small cell lung cancer: does PET make the difference? [Doctoral Thesis, Maastricht University]. Datawyse / Universitaire Pers Maastricht. https://doi.org/10.26481/dis.20080529ab

Document status and date:

Published: 01/01/2008

DOI:

10.26481/dis.20080529ab

Document Version:

Publisher's PDF, also known as Version of record

\section{Please check the document version of this publication:}

- A submitted manuscript is the version of the article upon submission and before peer-review. There can be important differences between the submitted version and the official published version of record.

People interested in the research are advised to contact the author for the final version of the publication, or visit the DOI to the publisher's website.

- The final author version and the galley proof are versions of the publication after peer review.

- The final published version features the final layout of the paper including the volume, issue and page numbers.

Link to publication

\footnotetext{
General rights rights.

- You may freely distribute the URL identifying the publication in the public portal. please follow below link for the End User Agreement:

www.umlib.nl/taverne-license

Take down policy

If you believe that this document breaches copyright please contact us at:

repository@maastrichtuniversity.nl

providing details and we will investigate your claim.
}

Copyright and moral rights for the publications made accessible in the public portal are retained by the authors and/or other copyright owners and it is a condition of accessing publications that users recognise and abide by the legal requirements associated with these

- Users may download and print one copy of any publication from the public portal for the purpose of private study or research.

- You may not further distribute the material or use it for any profit-making activity or commercial gain

If the publication is distributed under the terms of Article $25 \mathrm{fa}$ of the Dutch Copyright Act, indicated by the "Taverne" license above, 


\section{RADIOTHERAPY IN \\ NON-SMALL CELL LUNG CANCER}

Does PET make the difference?

Angela van Baardwijk 


\section{Cover illustration}

Repeated PET-CT imaging before, during and seventy days after fractionated radiotherapy in a patient with non-small cell lung cancer. The latter shows no uptake of FDG in the residual mass.

\section{Production}

Cover: Patricia Meijers-Bouts

Printing: $\quad$ Datawyse | Universitaire Pers Maastricht

ISBN 9789052787091

\section{Copyrights}

(C) Copyright A. van Baardwijk, Maastricht 2008

Chapter 2: $\quad$ Canc Treat Rev, Elsevier Ltd, 2006

Chapter 3: $\quad$ Eur J Canc, Elsevier Ltd, 2007

Chapter 4: $\quad$ Radiother Oncol, Elsevier Ireland Ltd, 2008

Chapter 5: Int J Radiat Oncol Biol Phys, Elsevier Inc, 2007

Chapter 6: Int J Radiat Oncol Biol Phys, Elsevier Inc, 2006

Chapter 7: $\quad$ Radiother Oncol, Elsevier Ireland Ltd, 2007

Chapter 8: Int J Radiat Oncol Biol Phys, Elsevier Inc, 2008

Chapter 9: $\quad$ Int J Radiat Oncol Biol Phys, Elsevier Inc, 2008

No part of this publication may be reproduced in any form without prior written permission of the holder of the copyrights. 


\section{RADIOTHERAPY IN \\ NON-SMALL CELL LUNG CANCER}

\section{Does PET make the difference?}

\section{PROEFSCHRIFT}

ter verkrijging van de graad van doctor aan de Universiteit Maastricht, op gezag van Rector Magnificus prof. mr. G.P.M.F. Mols, volgens het besluit van het College van Decanen in het openbaar te verdedigen op 29 mei 2008 om 14:00 uur

door

\section{Angela Antonetta Woutera van Baardwijk geboren op 23 september 1971 te Waalwijk}

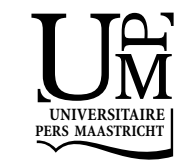




\section{Promotor}

Prof. dr. Ph. Lambin

\section{Copromotor}

Dr. D. De Ruysscher

\section{Beoordelingscommissie}

Prof. dr. J.M. van Engelshoven (voorzitter)

Prof. dr. A.L. Grosu

Prof. dr. G.J. Teule

Dr. G.P. ten Velde

The work presented in this thesis was supported by EC FP6 funding LSHC-CT-2004505785. The printing of this thesis was partially supported by Siemens Nederland NV. 


\section{Contents}

\section{Introduction}

Chapter 1 General Introduction 1

Chapter 2 FDG-PET in radiation treatment planning of NSCLC: a review 11

The biological meaning of FDG uptake in NSCLC

Chapter 3 FDG uptake correlates with survival, HIF-1a and GLUT-1

Chapter 4 Correlation of intra-tumour heterogeneity on FDG-PET with pathology: a feasibility study

The role of FDG-PET scan in radiotherapy of NSCLC

Chapter 5 PET-CT scan based auto-contouring in NSCLC 55

$\begin{array}{lll}\text { Chapter } 6 & \text { Tumour volume and tumour motion during radiotherapy } & 71\end{array}$

$\begin{array}{lll}\text { Chapter } 7 & \text { Time trends in FDG uptake during radiotherapy } & 83\end{array}$

Clinical implications: the role of individualized radiotherapy in NSCLC

Chapter 8 Individualized radiotherapy based on normal tissue constraints: in silico

Chapter 9 Individualized radiotherapy based on normal tissue constraints: feasibility

\section{Discussion}

Chapter 10 General discussion and future perspectives

Summary

Samenvatting

Publications

Dankwoord 



\section{Introduction ก}

C

(-

C.9-

Cran

-

C 



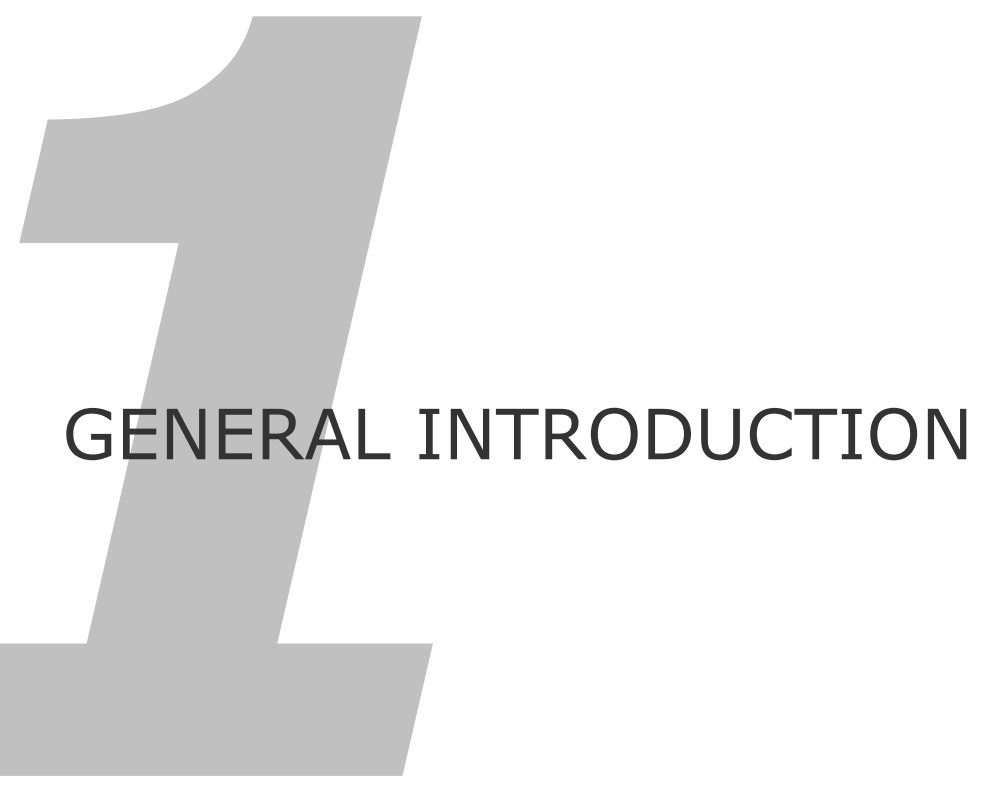





\section{Introduction}

\section{Epidemiology}

The incidence of cancer in Europe is increasing, with an estimated number of 3 million new cases a year ${ }^{1}$. Both in Europa and the US the most common form of cancer in men is prostate cancer $(20 \%-29 \%)$ followed by lung cancer $(15 \%-17 \%)$. In women, the most frequently diagnosed cancer is breast cancer (26\%-29\%), and respectively second (US) and fourth (Europe) in rank is lung cancer $(6 \%-15 \%)^{1,2}$. Lung cancer is the most common cause of cancer death in men, while it is the third cause of death from cancer in women in Europe. Especially with the ageing of the European population, cancer will remain a large problem in the near future. In the Netherlands the incidence of lung cancer is about 9000 a year $^{3}$, being the second most frequent cancer in men and the third in women (www.ikcnet.nl). The incidence is decreasing among Dutch men, but is still increasing in women ${ }^{4}$. In 2003 about 8800 patients died of lung cancer, being the leading cancer-related death in men and the second in women behind breast cancer.

Generally lung cancer can be divided in two main categories: non-small cell lung cancer (NSCLC) and small cell lung cancer (SLCL) with different clinical behaviour. NSCLC is the most frequent type of lung cancer and forms about $80 \%$ to $85 \%$ of all lung cancer cases $^{2}$. Furthermore, lung cancer can also be categorized according to pathologic subtype. The main subtypes of malignant epithelial lung tumours are squamous cell carcinoma, small cell carcinoma, adenocarcinoma, large cell carcinoma and adenosquamous carcinoma ${ }^{5}$. In men lung cancer consists mainly of squamous cell carcinoma, while the major part of lung cancer in women is adenocarcinoma ${ }^{6}$.

The prognosis of lung cancer is in general very poor, showing a 1-year survival of about $40 \%$ and a 5 -year survival of $15 \%{ }^{4,7,8}$.

\section{Diagnostics and staging}

NSCLC can be divided into several stages, based on the local extension, involvement of regional lymph nodes and the presence or absence of distant metastases. In general the TNM-classification is used for staging purposes ${ }^{6}$. In TNM the T gives information related to the extension of the primary tumour, the $\mathrm{N}$ stands for the invasion of regional (lymph) nodes and the $M$ for metastases. Currently, recommendations for the seventh revision of the TNM classification for lung cancer are being proposed ${ }^{9,10}$. Beside the TNM-system a staging system is employed (Table 1.1). Although the prognostic value of the current staging system has been demonstrated for surgical patients, its value is less clear in non-surgical patients ${ }^{9-11}$.

Standard diagnostic procedures to stage lung cancer consist of a physical examination, an X-ray of the thorax and laboratory investigations. Furthermore, a CT-scan of the thorax and a bronchoscopy, together with a histological or cytological biopsy of the tumour and/or involved lymph nodes, is performed. 


\begin{tabular}{|c|c|c|c|}
\hline & $T$ & $\mathrm{~N}$ & $M$ \\
\hline occult & $T x$ & NO & MO \\
\hline stage 0 & Tis & No & MO \\
\hline stage IA & T1 & No & MO \\
\hline stage IB & $\mathrm{T} 2$ & NO & MO \\
\hline stage IIA & $\mathrm{T} 1$ & N1 & MO \\
\hline \multirow[t]{2}{*}{ stage IIB } & $\mathrm{T} 2$ & $\mathrm{~N} 1$ & MO \\
\hline & T3 & NO & MO \\
\hline \multirow[t]{3}{*}{ stage IIIA } & $\mathrm{T} 1$ & N2 & MO \\
\hline & $\mathrm{T} 2$ & N2 & MO \\
\hline & T3 & $\mathrm{N} 1, \mathrm{~N} 2$ & MO \\
\hline \multirow[t]{2}{*}{ stage IIIB } & any $\mathrm{T}$ & N3 & MO \\
\hline & T4 & any $\mathrm{N}$ & MO \\
\hline stage IV & any $\mathrm{T}$ & any $\mathrm{N}$ & M1 \\
\hline
\end{tabular}

The ${ }^{18}$ F-fluoro-2-deoxy-glucose (FDG) Positron Emission Tomography (PET) is increasingly used in the diagnostic procedure of lung cancer. FDG-6-phosphate (FDG-6-P04), the end product of FDG, is accumulated in the cell and is a measure for the rate of glycolysis within the tumour. The preferential accumulation of FDG in neoplastic cells permits differentiation between benign and malignant tissue ${ }^{12}$. FDG-PET scan has a higher sensitivity, specificity and accuracy for detection of lymph node involvement and distant metastases in NSCLC than CT scan and therefore, results in a more accurate staging ${ }^{13-15}$. Therefore, PET has now become a standard imaging tool in the staging of lung cancer, and has resulted in a reduction in the number of thoracotomies up to $20 \%{ }^{16}$. The avoidance of futile thoracotomies is very important, since this procedure has its morbidity. Furthermore, patients referred for radical radiotherapy or chemoradiation may be found to already harbour metastatic disease. The finding of metastases on PET will therefore change the management from radical to palliative intent.

\section{Treatment modalities}

The treatment of NSCLC is dependent on both the general condition of the patient as well as the clinical tumour stage. For clinical stage I and II in fit patients, surgery is the first treatment of choice. Despite surgical resection, $50 \%$ to $60 \%$ of early stage patients relapse and will die from their lung cancer. For patients with early stage NSCLC with contraindications for surgery (medical inoperable patients) radiotherapy is commonly considered as the main treatment. In this group of patients stereotactic radiotherapy has experienced increasing importance, with promising initial results for local tumour control of $80 \%$ to $95 \%$ with 2 -year overall survival rates varying between $50 \%$ and $80 \%{ }^{17}$.

Stage III disease represents a very heterogeneous group of patients in which the optimal therapy is not clearly defined. A subset of stage IIIa patients might be considered surgical candidates after induction chemotherapy ${ }^{18}$. However, for patients with irresectable locally advanced tumours in a good general condition, radiotherapy alone or combined with chemotherapy is the principal choice of treatment. High local failure rates are observed in patients treated with radiotherapy alone or sequential chemoradiation. Therefore, several approaches have been applied to improve local tumour 
control and survival. These strategies include escalating the radiation dose, accelerated radiotherapy schedules and/or concomitant administration of chemotherapy and radiotherapy ${ }^{19-23}$. However, even with the concurrent administration of chemotherapy and radiation, local relapse occurs in about 30 percent of the patients as the initial site of failure ${ }^{24-26}$. And although radiation dose-escalation is associated with improved local control and overall survival, the possibilities for dose-escalation are often limited due to normal tissue constraints ${ }^{19}$.

In case of metastatic disease treatment mainly consists of palliative chemotherapy and supportive care. In this stage radiotherapy can play a meaningful role in the palliation of painful (bone) metastases.

\section{Improvement of radiotherapy in NSCLC}

Several issues contribute to the outcome in radiation treatment of NSCLC. The first one is the need of a correct staging; an incorrect staging will ultimately lead to under- or overtreatment of the patient. Therefore it is important to incorporate FDG-PET with its high diagnostic accuracy, in the staging procedure. Indeed, in $10 \%$ to $26 \%$ of cases, FDG-PET will change the intent of treatment from radical to palliative, because of the detection of distant metastases or locally advanced tumour, not suitable for radical treatment ${ }^{27,28}$. Secondly, it is important to define the target volume correctly, both for the primary tumour as well as for the involved lymph nodes. An incorrect defined target volume will lead to a geographical miss of the tumour and/or involved nodes during radiation, resulting in an increased risk of loco-regional recurrence. Both respiratory related tumour movements as well as changes during the series of fractionated radiotherapy will influence the localization and volume of the target volume, and should be taken into account for target volume delineation ${ }^{29}$. A third important issue contributing to the outcome is the total radiation dose delivered to the tumour. Not only the total radiation dose delivered, but also the time in which this dose is given is of utmost importance ${ }^{30,31}$. This is due to accelerated repopulation, which is usually observed about 3 weeks after start of radiotherapy ${ }^{32,33}$. Finally, in-vivo dosimetry using Electronic Portal Imaging Devices (EPID) and monitoring of the set-up of the patient are needed to assure the quality of the radiation treatment delivered ${ }^{34}$.

\section{Outline of thesis}

The first two chapters give an overview about the current role of PET-CT in the diagnostics and treatment of lung cancer. Chapter 1 contains the general introduction, whereas in Chapter 2 a review is given of the current status of FDG-PET in tumour volume definition in NSCLC in radiation treatment planning.

In this thesis several questions are addressed, which ultimately might lead to improvement of radiotherapy and hence outcome in NSCLC. 


\section{What is the biological meaning of FDG-uptake on PET scan?}

The maximal uptake of FDG in the primary tumour was consistently shown to be a significant, independent prognostic factor for survival, both after complete resection and after radical radiotherapy ${ }^{35-38}$. However, the actual mechanisms by which a high uptake of FDG leads to a worse prognosis are not well known. A high proliferative activity of the tumour might be related to a higher uptake of FDG, but contradicting findings have been observed regarding the correlation between proliferation and the uptake of $F G^{39,40}$. Hypoxia might be another important factor playing a role in the uptake of FDG, since it leads via the Hypoxia Inducible Factor-1a (HIF-1a) pathway to upregulation of several pathways, including the upregulation of glucose transporters ${ }^{41,42}$. Hypoxia leads to an increased rate of glycolysis, which in turn, increases the uptake of FDG. Moreover, hypoxia is an important cause of treatment failure in many tumours and has been related to a poor outcome ${ }^{43-46}$. Chapter 3 addresses the question whether the worse prognosis of NSCLC patients with a high FDG uptake is related to hypoxia. This chapter shows the results of a study, investigating the relation between the standardized uptake value (SUV) on FDG-PET scan and hypoxia related markers (HIF-1a and CAIX), a proliferation-related marker (Ki-67) and glucose transporters (GLUT-1 and GLUT-3) in 102 NSCLC patients.

Not only the background of a high uptake of FDG and its relation with a poor prognosis needs to be further investigated, but also the intra-tumour heterogeneity in the uptake of FDG as visualized on PET scan. This might reflect heterogeneity of ongoing pathophysiological processes within the tumour. More insight knowledge concerning the biological meaning of the intra-tumour heterogeneity might allow specific targeting, and will lead to further individualisation of the treatment of NSCLC ${ }^{47}$. In Chapter 4 the feasibility to correlate the intra-tumour heterogeneity as visualized on ${ }^{18}$ F-FDG PET with histology in an ex-vivo model is evaluated.

\section{Can FDG-PET-CT scan further improve the characterization and definition of the target volume?}

To achieve accurate radiation treatment planning a precise and consistent delineation of the target volume is necessary. However, there is large interobserver variability in the target volume delineation in NSCLC. The accuracy of target volume delineation can be improved using a standardized delineation protocol. Furthermore, integration of PET information into target volume delineation in NSCLC results in a reduction of interobserver variability compared with CT-based delineation ${ }^{48-51}$. Currently, target volume delineation is carried out manually. PET-based automatic contouring, based on e.g. Signal-to-Background ratios (SBR), might further improve target volume delineation and reduce interobserver variability ${ }^{52,53}$. Automatic contouring would be the ultimate method to use both in daily clinical practice, as well as for clinical studies. Chapter 5 describes the validation of a PET-CT scan based auto-contouring method and the influence of this auto-contour on interobserver variability in the delineation of the primary tumour and the involved lymph nodal volumes in NSCLC.

Usually treatment planning is mainly based on pre-treatment images only. The gross tumour volume (GTV) is commonly delineated on CT and consists of the visible tumour $^{54}$. To include microscopic disease a margin of 5 to $8 \mathrm{~mm}$ is taking into account ${ }^{55}$, 
creating the so-called clinical target volume (CTV). Furthermore, different factors like variations in size, shape and position of the CTV as well as uncertainties in patient position (set-up) are taken into account in the additional margins for the planning target volume (PTV) ${ }^{56}$. However, the target volume is not a static volume and in case of NSCLC, this will be influenced by tumour movement due to respiration as well as changes during fractionated radiation treatment ${ }^{29}$. A respiratory correlated CT scan provides information concerning the movement of the target volume due to respiration $^{57}$. This movement can be taken into account in the margins added from CTV to $\mathrm{PTV}^{58}$. Moreover, repeated imaging of the tumour during radiotherapy will contribute to a better definition of the target volume. Repeated PET-CT scanning can be used to track both changes in anatomics and tumour volume as well as metabolic changes and will give the opportunity to adapt treatment according to the changes. Chapter 6 and Chapter 7 are dedicated to the (individual) changes in tumour volume, tumour motion and maximal uptake of FDG during fractionated radiotherapy.

\section{Can the treatment of NSCLC be improved by individualization of radiotherapy?}

One of the approaches to further improve radiation treatment in NSCLC is doseescalation to improve loco-regional control and survival in NSCLC 19,22,59-61. However, dose escalation is limited by damage to normal tissues, like lung and spinal cord. Therefore, most dose escalation studies have included selected patients. In these studies they usually have categorized patients in groups and moved from one estimated iso-toxicity level to the next. However, the best achievable radiation schedule should be defined for each individual patient separately. Such a scheme would include the highest total tumour dose based on individualized normal tissue dose constraints for this patient; the so-called individualized radiation dose prescription. The last chapters of this thesis concern the application of an individualized maximal tolerable dose radiation prescription, using FDG-PET-CT for target volume delineation. Chapter 8 describes a theoretical dose modelling study. In this study the theoretical gain using the concept of an individualized dose prescription, based on normal tissue dose constraints, is evaluated. A comparison of 5 radiation schedules, including classical fractionation, hypofractionation and hyperfractionation combined with accelerated treatment is performed and the effect on estimated tumour control probability and the risk for pneumonitis are investigated. In Chapter 9 the clinical results, both toxicity and outcome, of a prospective feasibility study using an individualized dose prescription are described. In this study the radiation dose was individually escalated until a doselimiting normal tissue constraint.

Finally, Chapter 10 contains a discussion of the results mentioned in this thesis, their clinical implications and future directions. 


\section{References}

1. Ferlay J, Autier P, Boniol M, et al. Estimates of the cancer incidence and mortality in Europe in 2006. Ann Oncol 2007;18:581-592.

2. Jemal A, Siegel R, Ward E, et al. Cancer statistics, 2007. CA Cancer J Clin 2007;57:43-66.

3. van Dijck JA, Coebergh JW, Siesling S, et al. Trends of cancer in the Netherlands 1989-1998. In: Report of the Netherlands Cancer Registry: Vereniging van Integrale Kankercentra; 2002. pp. 24-25.

4. Bray F, Tyczynski JE, Parkin DM. Going up or coming down? The changing phases of the lung cancer epidemic from 1967 to 1999 in the 15 European Union countries. Eur J Cancer 2004;40:96-125.

5. Travis W, Brambilla E, Muller-Hermlink $\mathrm{H}$, et al. World Health Organization classification of tumours. Pathology and genetics of tumours of the lung, pleura, thymus and heart. Lyon: IARC Press; 2004.

6. Greene FL, Page DL, Fleming ID, et al. AJCC Cancer Staging manual. New York: SpringerVerlag; 2002.

7. Damhuis RAM, van Dijck JAAM, Siesling $S$, et al. Lung cancer and mesothelioma in the Netherlands 1989-1997. In: Vereniging van Integrale Kankercentra; 2000. pp. 20-23.

8. Sant M, Aareleid T, Berrino F, et al. EUROCARE-3: survival of cancer patients diagnosed 199094-results and commentary. Ann Oncol 2003;14 Suppl 5:v61-118.

9. Rusch VW, Crowley J, Giroux DJ, et al. The IASLC Lung Cancer Staging Project: proposals for the revision of the $\mathrm{N}$ descriptors in the forthcoming seventh edition of the TNM classification for lung cancer. J Thorac Oncol 2007;2:603-612.

10. Rami-Porta R, Ball D, Crowley J, et al. The IASLC Lung Cancer Staging Project: proposals for the revision of the $\mathrm{T}$ descriptors in the forthcoming (seventh) edition of the TNM classification for lung cancer. J Thorac Oncol 2007;2:593-602.

11. Mountain CF. Revisions in the International System for Staging Lung Cancer. Chest 1997; $111: 1710-1717$.

12. Phelps ME. Inaugural article: positron emission tomography provides molecular imaging of biological processes. Proc Natl Acad Sci U S A 2000;97:9226-9233.

13. Antoch G, Stattaus J, Nemat AT, et al. Non-small cell lung cancer: dual-modality PET/CT in preoperative staging. Radiology 2003;229:526-533.

14. van Tinteren $\mathrm{H}$, Hoekstra OS, Smit EF, et al. Effectiveness of positron emission tomography in the preoperative assessment of patients with suspected non-small-cell lung cancer: the PLUS multicentre randomised trial. Lancet 2002;359:1388-1393.

15. Luketich JD, Friedman DM, Meltzer CC, et al. The role of positron emission tomography in evaluating mediastinal lymph node metastases in non-small-cell lung cancer. Clin Lung Cancer $2001 ; 2: 229-233$.

16. van Tinteren $\mathrm{H}$, Hoekstra OS, Smit EF, et al. The implementation of PET in non-small-cell lung cancer in the Netherlands. Clin Oncol (R Coll Radiol) 2006;18:156-157.

17. Timmerman RD, Kavanagh BD, Cho LC, et al. Stereotactic body radiation therapy in multiple organ sites. J Clin Oncol 2007;25:947-952.

18. Rigas JR, Kelly K. Current treatment paradigms for locally advanced non-small cell lung cancer. J Thorac Oncol 2007;2 Suppl 2:S77-85.

19. Belderbos JS, Heemsbergen WD, De Jaeger K, et al. Final results of a Phase I/II dose escalation trial in non-small-cell lung cancer using three-dimensional conformal radiotherapy. Int $J$ Radiat Oncol Biol Phys 2006;66:126-134.

20. Kong FM, Ten Haken RK, Schipper MJ, et al. High-dose radiation improved local tumor control and overall survival in patients with inoperable/unresectable non-small-cell lung cancer: longterm results of a radiation dose escalation study. Int J Radioth Oncol Biol Phys 2005;63:324333.

21. Le Chevalier T, Arriagada R, Quoix E, et al. Radiotherapy alone versus combined chemotherapy and radiotherapy in nonresectable non-small-cell lung cancer: first analysis of a randomized trial in 353 patients. J Natl Cancer Inst 1991;83:417-423. 
22. Perez CA, Pajak TF, Rubin P, et al. Long-term observations of the patterns of failure in patients with unresectable non-oat cell carcinoma of the lung treated with definitive radiotherapy. Report by the Radiation Therapy Oncology Group. Cancer 1987;59:1874-1881.

23. Rowell NP, O'Rourke N P. Concurrent chemoradiotherapy in non-small cell lung cancer. Cochrane Database Syst Rev 2004:CD002140.

24. Furuse K, Fukuoka M, Kawahara M, et al. Phase III study of concurrent versus sequential thoracic radiotherapy in combination with mitomycin, vindesine, and cisplatin in unresectable stage III non-small-cell lung cancer. J Clin Oncol 1999;17:2692-2699.

25. Fournel P, Robinet $G$, Thomas $P$, et al. Randomized phase III trial of sequential chemoradiotherapy compared with concurrent chemoradiotherapy in locally advanced non-small-cell lung cancer: Groupe Lyon-Saint-Etienne d'Oncologie Thoracique-Groupe Francais de PneumoCancerologie NPC 95-01 Study. J Clin Oncol 2005;23:5910-5917.

26. Zatloukal $P$, Petruzelka L, Zemanova $M$, et al. Concurrent versus sequential chemoradiotherapy with cisplatin and vinorelbine in locally advanced non-small cell lung cancer: a randomized study. Lung Cancer 2004;46:87-98.

27. Dizendorf EV, Baumert BG, von Schulthess GK, et al. Impact of whole-body 18F-FDG PET on staging and managing patients for radiation therapy. J Nucl Med 2003;44:24-29.

28. Kalff V, Hicks RJ, MacManus MP, et al. Clinical impact of (18)F fluorodeoxyglucose positron emission tomography in patients with non-small-cell lung cancer: a prospective study. J Clin Oncol 2001;19:111-118.

29. Senan S, De Ruysscher D, Giraud P, et al. Literature-based recommendations for treatment planning and execution in high-dose radiotherapy for lung cancer. Radiother Oncol $2004 ; 71: 139-146$.

30. Saunders M, Dische S, Barrett A, et al. Continuous, hyperfractionated, accelerated radiotherapy (CHART) versus conventional radiotherapy in non-small cell lung cancer: mature data from the randomised multicentre trial. CHART Steering committee. Radiother Oncol 1999;52:137-148.

31. Fowler JF, Tome WA, Fenwick JD, et al. A challenge to traditional radiation oncology. Int $J$ Radiat Oncol Biol Phys 2004;60:1241-1256.

32. Bentzen SM, Saunders MI, Dische S. From CHART to CHARTWEL in non-small cell lung cancer: clinical radiobiological modelling of the expected change in outcome. Clin Oncol ( $R$ Coll Radiol) 2002; $14: 372-381$.

33. Hermens AF, Barendsen GW. Changes of cell proliferation characteristics in a rat rhabdomyosarcoma before and after x-irradiation. Eur J Cancer 1969;5:173-189.

34. Nijsten SM, Mijnheer BJ, Dekker AL, et al. Routine individualised patient dosimetry using electronic portal imaging devices. Radiother Oncol 2007;83:65-75.

35. Borst GR, Belderbos JS, Boellaard R, et al. Standardised FDG uptake: a prognostic factor for inoperable non-small cell lung cancer. Eur J Cancer 2005;41:1533-1541.

36. Downey RJ, Akhurst T, Gonen M, et al. Preoperative F-18 fluorodeoxyglucose-positron emission tomography maximal standardized uptake value predicts survival after lung cancer resection. $J$ Clin Oncol 2004;22:3255-3260.

37. Eschmann SM, Friedel G, Paulsen F, et al. Is standardised (18)F-FDG uptake value an outcome predictor in patients with stage III non-small cell lung cancer? Eur $\mathrm{J} \mathrm{NuCl} \mathrm{Med} \mathrm{Mol} \mathrm{Imaging}$ 2006;33:263-9.

38. Vansteenkiste JF, Stroobants SG, Dupont PJ, et al. Prognostic importance of the standardized uptake value on (18)F-fluoro-2-deoxy-glucose-positron emission tomography scan in nonsmall-cell lung cancer: An analysis of 125 cases. Leuven Lung Cancer Group. J Clin Oncol 1999;17:3201-3206.

39. Higashi K, Ueda Y, Yagishita M, et al. FDG PET measurement of the proliferative potential of non-small cell lung cancer. J Nucl Med 2000;41:85-92.

40. Vesselle $\mathrm{H}$, Schmidt RA, Pugsley JM, et al. Lung cancer proliferation correlates with [F18]fluorodeoxyglucose uptake by positron emission tomography. Clin Cancer Res 2000;6:3837-3844.

41. Vaupel P. The role of hypoxia-induced factors in tumor progression. Oncologist 2004;9 Suppl 5:10-17. 
42. Zhang JZ, Behrooz A, Ismail-Beigi F. Regulation of glucose transport by hypoxia. Am J Kidney Dis 1999;34:189-202.

43. Giatromanolaki A, Koukourakis MI, Sivridis E, et al. Expression of hypoxia-inducible carbonic anhydrase- 9 relates to angiogenic pathways and independently to poor outcome in non-small cell lung cancer. Cancer Res 2001;61:7992-7998.

44. Koukourakis MI, Bentzen SM, Giatromanolaki A, et al. Endogenous Markers of Two Separate Hypoxia Response Pathways (hypoxia inducible factor 2 alpha and carbonic anhydrase 9) Are Associated With Radiotherapy Failure in Head and Neck Cancer Patients Recruited in the CHART Randomized Trial. J Clin Oncol 2006.

45. Giatromanolaki A, Koukourakis MI, Sivridis E, et al. Relation of hypoxia inducible factor 1 alpha and 2 alpha in operable non-small cell lung cancer to angiogenic/molecular profile of tumours and survival. Br J Cancer 2001;85:881-890.

46. Swinson DE, Jones JL, Richardson D, et al. Carbonic anhydrase IX expression, a novel surrogate marker of tumor hypoxia, is associated with a poor prognosis in non-small-cell lung cancer. $J$ Clin Oncol 2003;21:473-482.

47. Bentzen SM. Theragnostic imaging for radiation oncology: dose-painting by numbers. Lancet Oncol 2005;6:112-117.

48. Ashamalla $H$, Rafla $S$, Parikh $K$, et al. The contribution of integrated PET/CT to the evolving definition of treatment volumes in radiation treatment planning in lung cancer. Int J Radiat Oncol Biol Phys 2005;63:1016-1023.

49. Caldwell $\mathrm{CB}$, Mah $\mathrm{K}$, Ung $\mathrm{YC}$, et al. Observer variation in contouring gross tumor volume in patients with poorly defined non-small-cell lung tumors on CT: the impact of 18FDG-hybrid PET fusion. Int J Radiat Oncol Biol Phys 2001;51:923-931.

50. Fox JL, Rengan R, O'Meara W, et al. Does registration of PET and planning CT images decrease interobserver and intraobserver variation in delineating tumor volumes for non-small-cell lung cancer? Int J Radiat Oncol Biol Phys 2005;62:70-75.

51. Steenbakkers RJ, Duppen JC, Fitton I, et al. Reduction of observer variation using matched CTPET for lung cancer delineation: a three-dimensional analysis. Int $\mathrm{J}$ Radiat Oncol Biol Phys 2006;64:435-448.

52. Daisne JF, Duprez T, Weynand B, et al. Tumor volume in pharyngolaryngeal squamous cell carcinoma: comparison at CT, MR imaging, and FDG PET and validation with surgical specimen. Radiology 2004;233:93-100.

53. Daisne JF, Sibomana M, Bol A, et al. Tri-dimensional automatic segmentation of PET volumes based on measured source-to-background ratios: influence of reconstruction algorithms. Radiother Oncol 2003;69:247-250.

54. ICRU report 50: Prescribing, recording, and reporting photon beam therapy. Bethesda; 1993.

55. Giraud P, Antoine M, Larrouy A, et al. Evaluation of microscopic tumor extension in non-smallcell lung cancer for three-dimensional conformal radiotherapy planning. Int J Radiat Oncol Biol Phys 2000;48:1015-1024.

56. ICRU report 62: Prescribing, recording, and reporting photon beam therapy. Bethesda; 1999.

57. Rietzel E, Pan T, Chen GT. Four-dimensional computed tomography: image formation and clinical protocol. Med Phys 2005;32:874-889.

58. Bosmans G, Buijsen J, Dekker A, et al. An "in silico" clinical trial comparing free breathing, slow and respiration correlated computed tomography in lung cancer patients. Radiother Oncol 2006;81:73-80.

59. Marks LB, Garst J, Socinski MA, et al. Carboplatin/paclitaxel or carboplatin/vinorelbine followed by accelerated hyperfractionated conformal radiation therapy: report of a prospective phase I dose escalation trial from the Carolina Conformal Therapy Consortium. J Clin Oncol $2004 ; 22: 4329-4340$.

60. Bradley J. A review of radiation dose escalation trials for non-small cell lung cancer within the Radiation Therapy Oncology Group. Semin Oncol 2005;32:S111-113.

61. Chen M, Hayman JA, Ten Haken RK, et al. Long-term results of high-dose conformal radiotherapy for patients with medically inoperable T1-3N0 non-small-cell lung cancer: is low incidence of regional failure due to incidental nodal irradiation? Int J Radiat Oncol Biol Phys 2005. 


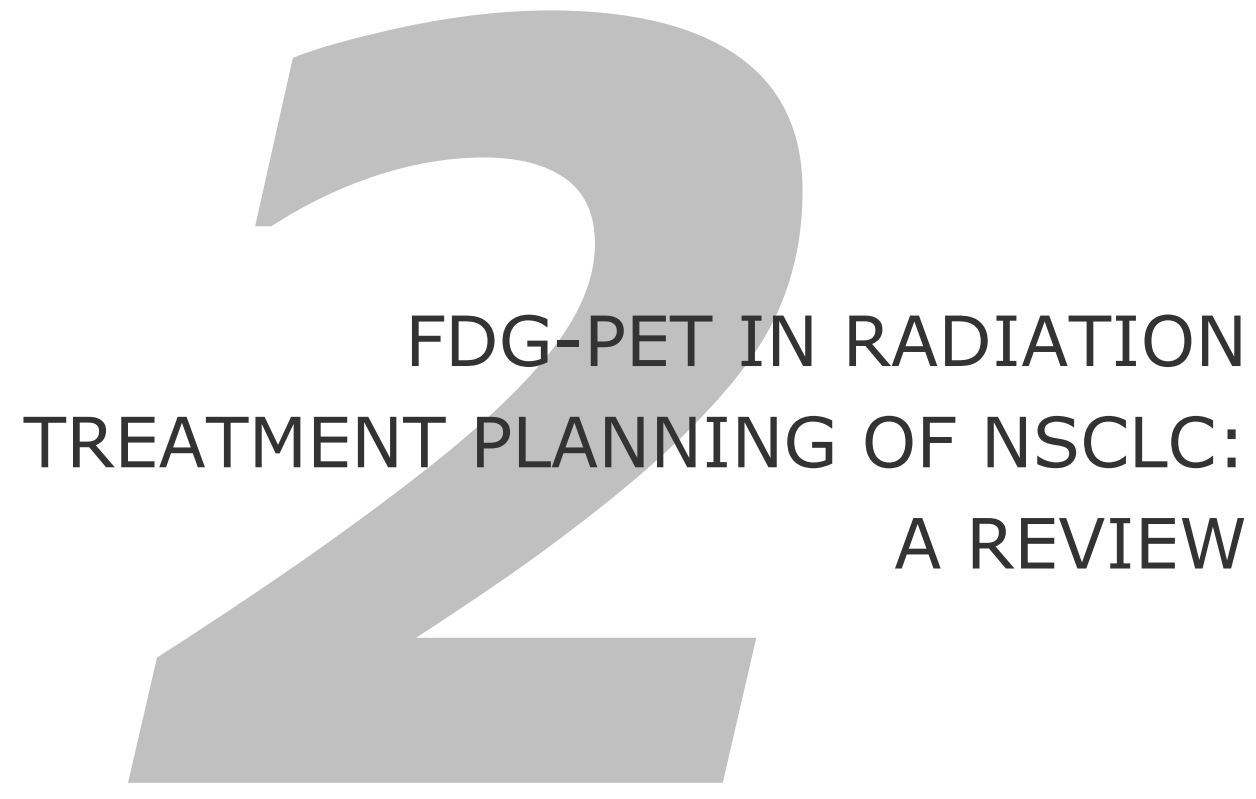

Adapted from:

A. van Baardwijk, B.G. Baumert, G. Bosmans, M. van Kroonenburgh, S. Stroobants, V. Gregoire, P. Lambin and D. De Ruysscher. The current status of FDG-PET in tumour volume definition in radiotherapy treatment planning.

Canc Treat Rev 2006;32(4):245-60 


\section{Abstract}

Purpose: Positron emission tomography (PET) scan, mainly using ${ }^{18}$ F-Fluorodeoxyglucose (FDG) as a tracer, is currently widely accepted as a diagnostic tool in oncology. It may lead to a change in staging and therefore in treatment management. PET can also be used to define the target volume in radiation treatment planning and to evaluate treatment response.

Materials and Methods: In this review, we focused on issues concerning the role of PET in target volume delineation in non-small cell lung cancer, both for the primary tumour and regional lymph nodes. A literature search was performed using MEDLINE. Furthermore, the following questions were addressed: does PET allow accurate tumour delineation and does it improve the outcome of radiotherapy, in terms of reduced toxicity or a higher tumour control probability?

Results: Most studies have shown a significant alteration in the target volume in almost half of the patients. The addition of PET resulted mainly in a decrease in target volume of $20 \%$ to $25 \%$. These changes were most often due to the exclusion of atelectasis and the inclusion or exclusion of lymph nodal stations. PET reduces the interobserver variability in target volume delineation. Moreover, PET might improve tumour coverage and reduces the volume of organs at risk irradiated.

Conclusion: Combined computer tomography (CT) and PET information seems to influence target volume delineation. Using (CT-)PET scan, interobserver variability is being reduced. In the field of lung cancer, incorporation of PET seems to improve tumour coverage and spare normal tissues, which may lead to less toxicity or the possibility to escalate dose. 


\section{Introduction}

Positron emission tomography (PET), mostly with ${ }^{18} \mathrm{~F}$-fluoro-deoxyglucose (FDG), and to a much lesser extend with ${ }^{11} \mathrm{C}$-methionine (MET) or ${ }^{11} \mathrm{C}$-choline, is increasingly used in oncology. The preferential accumulation of these radiotracers in neoplastic cells permits differentiation between benign and malignant tissue. In case of FDG, the end product of FDG, FDG-6-PO4 is retained in the cell, and is a measure for the rate of glycolysis in the tumour ${ }^{1}$. FDG-PET has for many cancer sites a higher sensitivity, specificity and accuracy for detection of tumour involvement than computer tomography (CT) or magnetic resonance imaging (MRI) and results in a more accurate staging of malignancies, both for the primary tumour, lymph nodes and distant metastasis $^{2-10}$. Since FDG uptake is closely related to biological characteristics of the tumour, treatment-induced changes resulting in tumour cell death or growth arrest, result in a subsequent reduction in FDG uptake, making this technique a sensitive marker of response. Several studies have investigated the role of PET for evaluation of treatment response and prediction of recurrence and/or survival after surgery, radiotherapy and chemotherapy, and have shown superiority for PET scan in several cases ${ }^{11-24}$.

Concerning the issue whether a post-radiotherapy PET scan could predict residual disease and hence outcome, several studies have shown that residual FDG uptake is often associated with a worse treatment outcome, although the timing of scanning and the clinical consequences are not yet obvious in all circumstances ${ }^{25-32}$. Despite this being very important, both of those issues are beyond the scope of this chapter.

Due to the high diagnostic accuracy of the PET, its role in radiotherapy has been investigated. From a theoretical point of view, the PET may influence radiotherapy by changing the $\mathrm{T}-$, the $\mathrm{N}$ - and the $\mathrm{M}$-staging with direct influence on treatment manage-

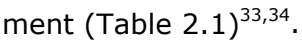

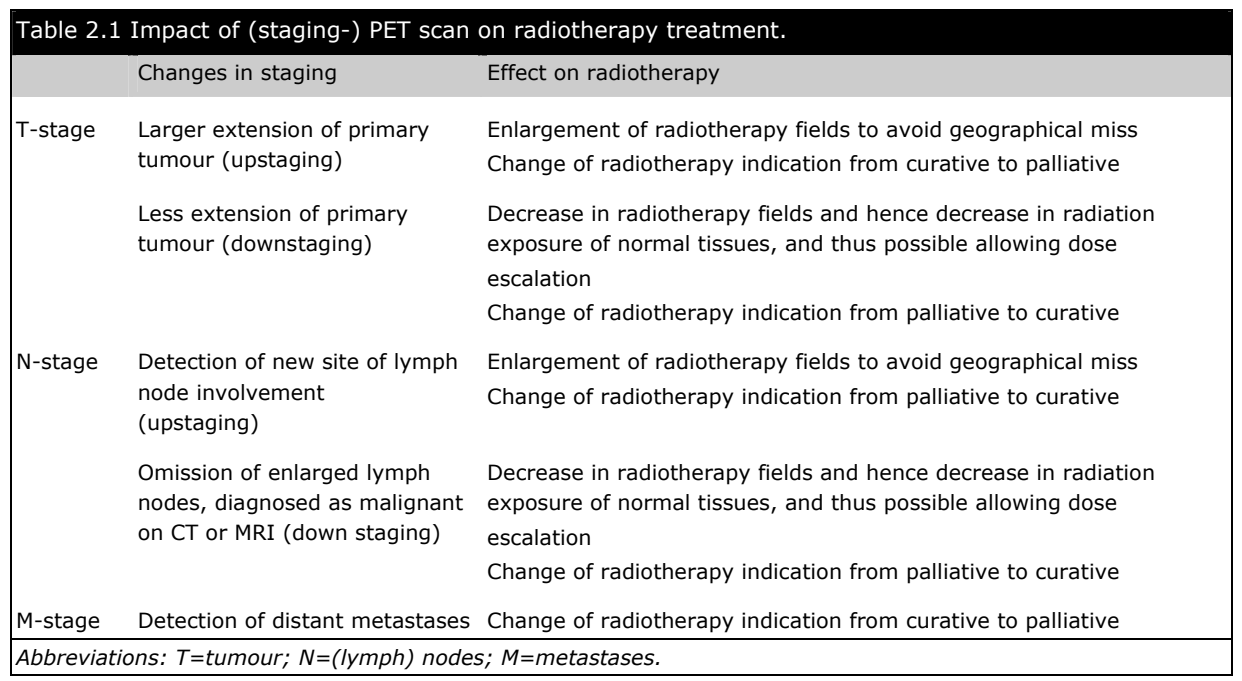


Indeed, in $10 \%$ to $30 \%$ of cases, FDG-PET changed the intent of treatment from radical to palliative, because of the detection of distant metastases or locally advanced tumour, not suitable for radical treatment ${ }^{34-38}$. In the case of new or additional PET findings, pathologic confirmation is needed before changing treatment. As there is a more accurate differentiation between tumour and non-malignant tissue compared with morphological imaging techniques, the integration of PET into radiotherapy planning may also influence delineation of target volumes and reduce the inter- and intraobserver variability ${ }^{34-51}$. This may also enable individualisation of radiotherapy strategies, as it provides information about specific biological characteristics of tumours. It may then lead to altered fractionation regimes and/or combination of radiotherapy with targeted drugs ${ }^{52,53}$.

The rational use of PET in radiation treatment planning depends on the qualities of PET(-CT). These qualities, like sensitivity, specificity, positive predictive value, negative predictive value and accuracy of PET and CT, are depending on the specific tumour site. For mediastinal lymp node staging in non-small cell lung cancer (NSCLC) both the sensitivity and specificity for PET(-CT) are higher than for CT alone. Whereas the CT has a sensitivity varying from $33 \%$ to $83 \%$, the sensitivity is $77 \%$ to $91 \%$ for PET(-CT), with a specificity between $67 \%$ and $90 \%{ }^{54-57}$.

Methods used to determine sensitivity and specificity of PET scanning differ between the mentioned studies. Sometimes numbers are specific either for the primary tumour, lymph nodes or metastatic disease or a combination of these. To further complicate the issue, numbers on lymph nodes may refer to individual nodes or nodal levels. More recently the combination of PET-CT has been compared side-by-side with CT and PET, $\mathrm{CT}$ alone and PET alone. The PET-CT combination has consistently been shown to have a higher sensitivity and accuracy compared with the individual modalities. This holds both for the primary tumour and lymph node metastases ${ }^{9}$. It is beyond the scope of this chapter to discuss in depth how to use PET (or PET-CT) according to their intrinsic values of sensitivity and specificity. An editorial has been written on that subject ${ }^{58}$.

In this study we will only address the question of how PET scan influences radiotherapy planning in stricto senso, i.e. how will this technique change the definition of target volumes in patients selected for radiotherapy with curative intent. Ideally information about the delineation of the Gross Tumour Volume (GTV) and/or Clinical Target Volume (CTV) should be given. In the case of Planning Target Volume (PTV) margins for movement and set-up variations are also taken into account. This makes it more difficult to compare these volumes between different centres, as they are dependent on the radiation and immobilisation techniques used.

We will therefore focus on the following questions:

- Does PET scanning allow accurate tumour delineation?

- Does PET scanning change Gross Tumour Volume (GTV), Clinical Target Volume and/or the Planning Target Volume (PTV), both for the primary tumour and the local and regional lymph nodes?

- Does PET scanning allow improvement of treatment outcome? 


\section{Materials and Methods}

A literature search was performed on MEDLINE with the key words (FDG-)PET, lung cancer, NSCLC, radiotherapy, radiation, oncology, cancer, treatment, therapy, planning, target volume, Gross Tumour Volume (GTV), Clinical Target Volume (CTV), Planning Target Volume (PTV), staging, contouring, delineation, variability, observer, intra-observer, interobserver, toxicity, dose escalation, recurrence, fields and permutations of these. Only articles, published before July $31^{\text {st }} 2007$, in English were studied. Most studies used FDG as a tracer, and in this article PET will imply FDG-PET, unless otherwise stated. Studies were omitted when they did not include radiotherapy patients, studied other radiation qualities than photons (e.g. protons), only compared data on staging for distant metastases, only addressed basic research questions or only provided data on the proportion of patients in which the indication for radiotherapy changed from radical to palliative intent for this was deemed being an extension of staging.

\section{Results}

\section{Does PET scanning allow accurate tumour delineation?}

The major drawback of PET is the lack of anatomic detail. Combining PET and CT or MRI-scan will be needed to provide anatomical information. The exact meaning of PET signal is not known and in general, studies evaluating the correlation between target volume delineation based on PET and pathological data are missing. To date, only few studies in head-and-neck cancer compared PET, CT and MRI based delineation of the primary tumour with pathologic findings, and showed a complex relation ${ }^{59,60}$. Studies with similar design in NSCLC are awaited to provide a better idea about the definite role of PET scanning in radiation treatment planning.

Integrated PET-CT-scan for treatment planning might improve the standardization of volume delineation when compared with $\mathrm{CT}$ alone. With anatomic imaging modalities, like $\mathrm{CT}$, the boundaries of the tumour are difficult to define, especially in patients with atelectasis or displacement of normal tissue. Consequently, a significant interobserver variation is found for imaged based (CT and MRI) contouring of GTV at different tumour sites ${ }^{61-64}$. By adding PET-information not only the mean volume of GTV is reduced, but there is also a reduction of the standard deviation ${ }^{35}$. The ratio of largest to smallest GTV based on PET-CT information shows a narrower range compared with CT alone. Caldwell et al. found a decrease of this ratio in 23 out of 30 patients $(77 \%)$ with NSCLC with a mean ratio of largest to smallest GTV for CT-alone of 2.31 and for PET-CT of 1.56, a significant decrease ${ }^{42}$. Co-registration of CT and PET images even further reduces interobserver variability compared with non-registered images in the delineation of lung tumours ${ }^{47,65}$. Steenbakkers et al. investigated interobserver variation in NSCLC, comparing the CT and CT-PET based delineation of 22 lung tumours by 11 radiation oncologists. They observed a reduction in observer variation from a standard deviation of $1.0 \mathrm{~cm}$ for CT to $0.4 \mathrm{~cm}$ for matched CT-PET. Moreover, they found a reduction in the mean delineation time (12 versus 16 minutes $)^{43}$. 
Although interobserver variability is still seen with (CT-)PET, it seems to be minimalised compared with conventional imaging methods.

Most studies using PET for target volume delineation use the visual interpretation of PET images. More and more automatic segmentation based on a certain intensity level, such as Standardized Uptake Value (SUV)-level, a percentage of the maximal SUV or the source-to-background ratio is used ${ }^{34,60,66-71}$. Which is the best method to be used still needs to be further evaluated ${ }^{72}$. One has to be aware however that SUV, defined as the average activity per unit volume normalized to the injected dose and patient body weight, is influenced by different factors making it only reliable in a standardized setting. Factors that interfere with SUV quantification can grossly be divided into three areas: first tissue activity factors such as the shape of the Region of Interest (ROI), the partial-volume and spill over effects, attenuation correction, reconstruction methods and parameters for scanner type. Secondly it is influenced by tissue state factors amenable for corrections such as the time of SUV evaluation and competing transport effects and finally by the normalization factor (body size, body surface area, lean body mass $)^{73-76}$. As pathologic examination is the golden standard, more results comparing PET-findings with pathology are needed. This information will provide us with information about the definite role of PET scanning in target volume delineation and radiation treatment planning.

\section{Does PET scanning change Gross Tumour Volume (GTV) and the Planning Target Volume (PTV), both for the primary tumour and the local and regional lymph nodes in NSCLC?}

\section{Primary tumour}

FDG-PET scanning plays an important role to differentiate between malignant and benign pulmonary lesions ${ }^{77}$. Several articles have been published about the integration of the FDG-PET information into radiotherapy planning in NSCLC, concerning GTV delineation $34-36,39,40,44-51$. PET data were incorporated into CT-based radiotherapy treatment planning by visual comparison or with image co-registration. Both an increase and decrease in target volumes, GTV and PTV, have been described. Most studies showed a treatment volume modification in about half of the patients ${ }^{39,44-48}$. Bradley et al. found a GTV contour change in 17 out of 24 patients (58\%): an increase in $12 \%$ and a decrease in $46 \%{ }^{48}$. Ashmalla and co-writers found in $10 / 19$ patients (52\%) a treatment volume modification larger than $25 \%$ in stage II-IIIB NSCLC. In half of these cases an increase of the GTV was observed ${ }^{47}$. Deniaud-Alexandre et al. observed a decrease in GTV in 21 out of 101 patients and an increase in GTV in 24 patients ${ }^{46}$, while Grills and co-workers observed in $48 \%$ of patients a smaller GTV and in $33 \%$ a larger GTV on PET compared with $\mathrm{CT}^{40}$. Looking at more quantitative information on the effects of integrating PET data into CT planning for NSCLC, several studies describe the influence on GTV and/or PTV. Although most studies show a decrease in target volume due to the addition of PET (mean decrease of $8 \%$ till $29 \%$ ) $^{35,48,49,78}$, Erdi et al. and Munley et al. both observed an increase in mean target volume ${ }^{50,51}$. The largest series published by Deniaud-Alexandre et al., showed a decrease in $20 \%$ of patients (mean decrease of $42 \% \pm 8 \%$ ) and an increase in $24 \%$ of patients (mean increase of $26.5 \% \pm 19 \%)^{46}$. 
In general, the major cause for a decrease in target volume is the exclusion of atelectasis, which seems easier to diagnose with PET-CT compared with CT alone. An increase in GTV was mainly caused the detection of additional mediastinal nodal disease on PET compared with CT.

\section{Lymph nodes}

In view of the higher diagnostic accuracy of FDG-PET compared with CT for staging the mediastinum ${ }^{54-57,79}$, the incorporation of PET in radiotherapy planning of patients with NSCLC was investigated. In the study of Vanuytsel et al. CT and PET positive nodes were compared with the golden standard, pathologic examination, in 988 lymph nodes $^{78}$. They found in a subgroup of 73 patients, that on CT criteria alone the GTV would include all pathologic nodes in $75 \%$ of patients $(55 / 73)$, while based on PET-CT data this was $89 \%(p=0.005)$. Kiffer et al. found in 4 out of 15 patients $(26.7 \%)$ that the mediastinal lymph nodes showed significant FDG uptake on the PET, whilst no abnormality was found on $\mathrm{CT}^{80}$. Giraud et al. found similar results: in 4 out of 12 patients, PET positive lymph nodes (in 2 cases mediastinal) were negative on CT, leading to a modified treatment plan ${ }^{81}$.

In NSCLC most groups only irradiate the involved mediastinal lymph nodes based on CT scans ${ }^{82-84}$. Van der Wel et al. showed in a group of 21 patients with clinical CT stage N2/N3 MO NSCLC that the GTV of the lymph nodes decreased from $13.7 \pm 3.8 \mathrm{~cm}^{3}$ based on $\mathrm{CT}$ to $9.9 \pm 4.0 \mathrm{~cm}^{3}$ on PET-CT $(p=0.011)^{85}$. Moreover, it was shown that irradiating only PET positive mediastinal lymph nodes could be safely applied in clinical practice. In a phase II study, only one isolated failure occurred in a group of 44 patients ${ }^{86}$. These results were confirmed by Belderbos et al. ${ }^{87}$.

A summary of the given literature about the influence of the PET-scan on the measured target volumes in NSCLC is given in Table 2.2.

\begin{tabular}{|c|c|c|c|c|c|c|c|}
\hline & No & \multicolumn{2}{|c|}{$\begin{array}{l}\text { CT based target } \\
\text { volume (in } \mathrm{cc} \text { ) }\end{array}$} & \multicolumn{2}{|c|}{$\begin{array}{l}\text { PET(-CT) based target } \\
\text { volume (in } \mathrm{cc} \text { ) }\end{array}$} & & Conclusions \\
\hline & & \multicolumn{4}{|c|}{ Primary tumour } & \multirow{4}{*}{$p=0.002$} & \\
\hline $\begin{array}{l}\text { Vanuytsel, } \\
2000\end{array}$ & 10 & PTV $_{C T}$ & 579.0 & PTV $_{\text {PET }}$ & 402 & & $\begin{array}{l}\text { PTV }_{\text {PET }} \text { was significant } \\
\text { smaller than } \text { PTV }_{C T}\end{array}$ \\
\hline \multirow{2}{*}{$\begin{array}{l}\text { Ciernik, } \\
2003\end{array}$} & 6 & $\mathrm{GTV}_{\mathrm{CT}}$ & 36.1 & $\mathrm{GTV}_{\mathrm{PET}}$ & 27.8 & & \\
\hline & & PTV $_{\text {CT }}$ & 444.4 & PTV $_{\text {PET }}$ & 399.7 & & Mean change in PTV of $26 \%$ \\
\hline $\begin{array}{l}\text { Bradley, } \\
2004\end{array}$ & 24 & $\mathrm{GTV}_{\mathrm{CT}}$ & 111.3 & $\mathrm{GTV}_{\mathrm{PET}}$ & 99.8 & \multirow[t]{2}{*}{$p=n s$} & $\begin{array}{l}\text { No difference in } \mathrm{GTV}_{\mathrm{CT}} \text { and } \\
\text { GTV }_{\mathrm{PET}} \text { in the total group }\end{array}$ \\
\hline \multirow[t]{2}{*}{$\begin{array}{l}\text { Deniaud- } \\
\text { Alexandre, } \\
2005\end{array}$} & 92 & PTV $_{C T}$ & 361.0 & PTV & 347.5 & & $\begin{array}{l}\text { Increase in PTV in } 25 \% \text { of } \\
\text { patients (mean increase of } \\
35 \% \text { ) and a decrease in } 22 \% \\
\text { of patients (mean reduction } \\
\text { of } 27 \% \text { ) }\end{array}$ \\
\hline & & \multicolumn{2}{|c|}{ Lymph nodes } & & & & \\
\hline $\begin{array}{l}\text { Van der Wel, } \\
2005\end{array}$ & 21 & $\mathrm{GTV}_{\mathrm{CT}}$ & 13.7 & GTV $_{\text {PET-CT }}$ & 9.9 & $p=0.011$ & $\begin{array}{l}\text { GTV }_{\text {PET-CT }} \text { was significant } \\
\text { smaller than } \text { GTV }_{\text {CT }}\end{array}$ \\
\hline
\end{tabular}


In conclusion, most studies have shown a significant alteration in the target volume in $25 \%$ to $50 \%$ of patients with NSCLC. Mostly a decrease in target volume was noticed, with a change of about 20 to $25 \%$, when adding PET information for radiotherapy planning. The main causes for an increase in target volume are a larger primary tumour and most of all inclusion of additional nodal disease. The major cause for a decrease in target volume was the ability of PET to exclude atelectasis. Nevertheless, there are no pathologic data confirming that atelectatic regions do not contain any tumour cells. A correlation between pathologic examination and image-based delineation has only been performed for CT, but not for PET ${ }^{88-90}$. In NSCLC, PET-CT has a high diagnostic accuracy for detecting mediastinal lymph nodes and adding PET information for radiation treatment planning will lead to modified plans. In clinical studies, it was shown that it was safe to only irradiate PET positive mediastinal lymph nodes ${ }^{86,87}$. Further research to correlate target volumes based on PET and the golden standard of pathology needs to be done to validate the role of PET in delineating the tumour volumes in lung cancer.

\section{Does PET scanning allow improvement in treatment?}

A better delineation of the target volume might lead to an improvement in treatment. In case of radiotherapy with curative intent, the dose to normal tissues is usually the dose-limiting factor. Using the combined information of PET and CT can probably decrease the dose in critical organs at risk. When looking at normal tissues few studies have given limited information on Dose Volume Histograms (DVH) with and without FDG-PET $31,34,39,40,45,46,50,78,81,85$. Several studies showed no statistical significant differences in the DVH concerning percent lung volume receiving 20 Gy or more ( $V_{20}$ lung) comparing plans on base of CT data only or with additional PET information ${ }^{34,39,45}$. However, both Giraud et al. and Vanuytsel et al. found a reduction in $V_{20}$ lung. In the study of Giraud et al. $(n=12)$ there was a marked reduction of the $V_{20}$ lung by an average of $22.8 \%$. In only 1 patient there was an increased volume (5\%) of healthy lung irradiated, but in 4 other patients the $V_{20}$ lung was decreased with a maximal reduction of almost $50 \%{ }^{81}$. Vanuytsel et al. performed in 10 selected cases a dosimetric study, leading to a reduction of $\mathrm{V}_{20}$ lung of $27 \%(8-58 \%)^{78}$. Deniaud-Alexandre and co-workers observed an increase in $V_{20}$ by PET in 15 out of 81 patients (mean increase of $154 \%$ ) and a reduction in $V_{20}$ in 22 patients (mean reduction of $\left.19 \%\right)^{46}$. Van der Wel et al. showed in a modelling study, consisting of a group of 21 patients with NSCLC, that the use of combined CT and PET information leaded to a decrease in mean $V_{20}$ lung from $24.9 \%$ to $22.3 \%(p=0.012)$ and in the mean lung dose (MLD) from $14.7 \mathrm{~Gy}$ to $13.6 \mathrm{~Gy}(p=0.004)^{85}$. Considering the $V_{45}$ and $V_{55}$ oesophagus the same pattern was shown in this study: a decrease from $45.2 \%$ to $34.0 \%$ for $V_{45}(p=0.012)$ and from $30.6 \%$ to $21.9 \%$ for $\mathrm{V}_{55}(\mathrm{p}=0.004)$. However, Ceresoli et al. and Grills et al. did not observe a change in respectively $D_{\max }$ and $D_{\text {mean }}$ for the esophagus. Moreover, Deniaud-Alexandre et al. investigated changes in the percentage of total heart volume receiving more than $36 \mathrm{~Gy}$ in a group of 81 patients ${ }^{46}$. They observed an increase in 8 patients and a decrease in 14 patients, while Certolosi et al. observed a non-significant decrease in $D_{\text {mean }}$ of the heart ${ }^{45}$. Looking at the impact of dose on the spinal cord in NSCLC patients Mah et al. found a reduced dose on the spinal cord when adding FDGPET information. The ratio of the maximum cord dose for the PET-CT plan/dose for the CT plan varied from 0.05 to 24 . A significant lower average maximum dose for the spinal cord was found for the PET-CT plans compared with the CT plans ${ }^{34}$. This finding 
was confirmed by Ceresoli et al. ${ }^{45}$, while in the series published by Deniaud-Alexandre et al. the mean spinal dose was unchanged comparing PET-CT to CT based planning ${ }^{46}$. Erdi et al. calculated the Normal Tissue Complication Probability (NTCP) in patients with stage I-III NSCLC. No difference was seen in the highest NTCP for lung when using combined PET-CT planning (22\%) or CT only $(21 \%)^{50}$. Grills and colleagues observed a lung NTCP of $18 \%$ for the CT-based planning, compared with $15 \%$ for the PET-based planning ${ }^{40}$. They also investigated the esophagus NTCP and observed a NTCP of $20.2 \%$ for CT-based planning and $18.7 \%$ for PET-based planning.

Concerning the risk of geographical miss, MacManus et al. showed that in 3 out 10 patients CT-based planning ultimately would lead to a geographical miss if PET was not used $^{39}$. Kiffer et al. observed that if radiation ports based on CT alone were projected on the PET based target volumes, this would result in an inadequate coverage in $26 \%$ of patients ${ }^{80}$. Moreover, van der Wel et al. investigated the theoretical gain in TCP using the same toxicity levels for the CT-based and the PET-CT based plans ${ }^{85}$. The dose could be raised from $56.0 \mathrm{~Gy}$ to $71.0 \mathrm{~Gy}(p=0.038)$, leading to an increase in estimated tumour control probability (TCP) from about $13 \%$ to $18 \%$. Using a combined PET-CT simulator these results were confirmed, showing a decrease in exposure to oesophagus and lung. An increase in dose would lead to calculated TCP's rising from $6.3 \%$ for CT planning to $24.0 \%$ for PET-CT planning ${ }^{91}$.

In patients with NSCLC, considered for radiation treatment with curative intent, the assessment of tumour localization in loco-regional lymph nodes, by means of PET, might improve tumour coverage. In a subgroup of patients, the volume of organs at risk irradiated, like lung, oesophagus, heart and spinal cord, can be reduced. This may lead to a decrease in toxicity or accepting the same level of toxicity, this can lead to dose-escalation with an expected gain in tumour control probability.

\section{Discussion}

Using combined PET-CT information seems to influence target volume delineation. The main problem is that only few studies have been performed comparing delineation based on PET with the golden standard of pathologic examination. In head-and-neck cancer a larger GTV was found using PET data compared with the macroscopic examination although there was an underestimation considering the extension in the mucosa ${ }^{59}$. Most studies performed in NSCLC show a significant alteration in target volume by incorporating PET information into treatment planning $31,39,45,46,50,78,81,85$. PET seems to help in differentiating between atelectasis and tumour and in identifying positive mediastinal nodes. In clinical studies it was shown that irradiating only the PET positive mediastinal lymph nodes is safe $^{86,87}$. However, more data are needed to further investigate the explicit role of PET in delineating gross target volumes in radiation treatment planning. Using (CT-)PET scan interobserver variability is still seen, but it seems to be minimalised compared with conventional imaging methods ${ }^{43,47,65}$. Preliminary results show that incorporating PET data into treatment planning, probably will improve tumour coverage. In a subgroup of patients, the volume of organs at risk irradiated can be reduced and this gives the opportunity to escalate doses without increasing the complication risk. However, the field of incor-porating PET data into 
radiotherapy planning is a rapidly evolving, meaning that information provided today may be outdated tomorrow.

However, PET scanning has some drawbacks. Ideally imaging modalities or a combination of different tools should show all tumour nodules, which are present in a patient. One of the problems is that at the moment no modality does adequately detect subclinical disease. Margins for subclinical disease will be needed using PET, as they are needed in CT and MRI. The PET has a relative low spatial resolution of 6 to $7 \mathrm{~mm}$. Although a lot of effort is put into the improvement of the spatial resolution, it is physically limited to about $2 \mathrm{~mm}$, and microscopic disease may thus still be missed on FDG-PET images. Technical advances in software, image fusion and image processing might improve interpretation of images.

One of the other shortcomings of PET scanning is that the exact borders of tumours on the PET are not well defined, making visual delineation error prone. Therefore, software has been developed, which will create a contour automatically when a certain intensity or SUV value is given. However, the literature is not clear about the intensity level that should be chosen to contour a tumour, should this be $40 \%$ or $50 \%$ of the maximal intensity ? $^{34,48,66-72,92}$ Another method developed for automatic volume segmentation is based on the relationship between source-to background ratio and the activity level as has been described by Daisne et al. ${ }^{69}$. This last method looks promising in relation with pathologic examination in laryngeal cancer ${ }^{59}$. A background based thresholding seems also feasible in the delineation of lymph nodes in $\mathrm{NSCLC}^{93}$. However, since e.g. scanning protocols and SUV levels are not standardised, data from literature still need to be validated in individual departments, before introducing them into clinical practice.

Both organ and tumour movements influence PET findings. In using image fusion of CT data and PET data, it has to be taken into account, that CT data are more rapidly acquired, whereas a PET dataset needs a longer acquisition time, which leads to fusion problems. E.g. in the thoracic region respiratory motion causes image artefacts. Respiration-correlated (RC) acquisition of both CT and PET can tackle part of this problem by reducing the smearing and improving the accuracy of co-registration of PET and CT data. The attenuation correction will be performed more accurately and hence SUV will be defined more precisely.

Different tracers are and will become available to identify different kind of processes ongoing in tissue. For example, the PET tracer ${ }^{18} \mathrm{~F}$-fluoromisonidazole (F-MISO) allows non-invasive assessment of tumour hypoxia, while $3^{\prime}$-Deoxy- $3^{\prime}-{ }^{18} \mathrm{~F}$-fluorothymidine (F-FLT) is developed to investigate tumour cell proliferation. Most tracers have a relatively short half-life, e.g. ${ }^{11} \mathrm{C}$ has a half-life time of 20 minutes, compared with 120 minutes for ${ }^{18} \mathrm{~F}$. This short half-life time influences the clinical availability for centres without a cyclotron ${ }^{1}$. The development of new PET radiopharmaceuticals may in the future allow in vivo detection of tumour biological properties, like malignant potential, hypoxia and eventually responsiveness to treatment. This would a provide information leading to a further individualization of cancer treatment. 
PET-CT may also play a role in the response of the tumour to different treatment modalities, both during and after treatment. Especially evaluation of response early during treatment will give the possibility to adapt radiotherapy to the PET findings.

Molecular imaging will be part of the future of radiation treatment planning. The advances in technology in imaging technology and the development of new tracers, which can be used to evaluate in a non-invasive way biological characteristics, together with the possibility to spare more normal tissues or to escalate tumour dose, will lead to more individualized cancer treatment and hopefully to better treatment results in radiation oncology.

\section{Conclusions}

The PET is currently more and more accepted as a diagnostic tool in oncology and is increasingly used in defining the target volume in radiation treatment planning in NSCLC. Looking at issues concerning the role of PET in treatment planning, combined PET-CT information seems to influence target volume delineation. However, data on the confirmation of the relation between delineation based on (CT-)PET and pathologic examination are scarce. More research is needed to address the question whether PET does allow accurate tumour delineation in regard to pathological tumour extension. As to the question whether PET improves outcome of radiotherapy, theoretical studies show that using PET information in treatment planning can lead to sparing of normal tissues and, by escalating dose, to a higher tumour control probability. Additionally, PET can add knowledge on different biological characteristics of the tumour itself. This biological information might be used in the future to delineate a biological target volume and to adapt radiotherapy treatment, both in terms of volume as well as in dose. More basic research and clinical confirmation of theoretical studies are awaited, before the definite role of PET in radiation treatment planning becomes clear. 


\section{References}

1. Phelps ME. Inaugural article: positron emission tomography provides molecular imaging of biological processes. Proc Natl Acad Sci U S A 2000;97:9226-9233.

2. Kantorova I, Lipska L, Belohlavek O, et al. Routine (18)F-FDG PET preoperative staging of colorectal cancer: comparison with conventional staging and its impact on treatment decision making. J Nucl Med 2003;44:1784-1788.

3. Follen M, Levenback CF, Iyer RB, et al. Imaging in cervical cancer. Cancer 2003;98:2028-2038.

4. Rasanen JV, Sihvo EI, Knuuti MJ, et al. Prospective analysis of accuracy of positron emission tomography, computed tomography, and endoscopic ultrasonography in staging of adenocarcinoma of the esophagus and the esophagogastric junction. Ann Surg Oncol 2003;10:954-960.

5. Young CS, Young BL, Smith SM. Staging Hodgkin's Disease with 18-FDG PET. Comparison with CT and Surgery. Clin Positron Imaging 1998;1:161-164.

6. Antoch G, Stattaus J, Nemat AT, et al. Non-small cell lung cancer: dual-modality PET/CT in preoperative staging. Radiology 2003;229:526-533.

7. Luketich JD, Friedman DM, Meltzer CC, et al. The role of positron emission tomography in evaluating mediastinal lymph node metastases in non-small-cell lung cancer. Clin Lung Cancer $2001 ; 2: 229-233$.

8. Antoch G, Vogt FM, Freudenberg LS, et al. Whole-body dual-modality PET/CT and whole-body MRI for tumor staging in oncology. Jama 2003;290:3199-3206.

9. Antoch G, Saoudi N, Kuehl H, et al. Accuracy of whole-body dual-modality fluorine-18-2-fluoro2-deoxy-D-glucose positron emission tomography and computed tomography (FDG-PET/CT) for tumor staging in solid tumors: comparison with CT and PET. J Clin Oncol 2004;22:4357-4368.

10. Kresnik E, Mikosch P, Gallowitsch HJ, et al. Evaluation of head and neck cancer with 18F-FDG PET: a comparison with conventional methods. Eur J Nucl Med 2001;28:816-821.

11. Greven KM, Williams DW, 3rd, McGuirt WF, Sr., et al. Serial positron emission tomography scans following radiation therapy of patients with head and neck cancer. Head Neck 2001;23:942-946.

12. Halfpenny W, Hain SF, Biassoni L, et al. FDG-PET. A possible prognostic factor in head and neck cancer. Br J Cancer 2002;86:512-516.

13. Rege S, Safa AA, Chaiken L, et al. Positron emission tomography: an independent indicator of radiocurability in head and neck carcinomas. Am J Clin Oncol 2000;23:164-169.

14. Allal AS, Dulguerov $P$, Allaoua $M$, et al. Standardized uptake value of 2-[(18)F] fluoro-2-deoxy$\mathrm{D}$-glucose in predicting outcome in head and neck carcinomas treated by radiotherapy with or without chemotherapy. J Clin Oncol 2002;20:1398-1404.

15. Ahuja V, Coleman RE, Herndon J, et al. The prognostic significance of fluorodeoxyglucose positron emission tomography imaging for patients with nonsmall cell lung carcinoma. Cancer 1998;83:918-924.

16. Allal AS, Slosman DO, Kebdani T, et al. Prediction of outcome in head-and-neck cancer patients using the standardized uptake value of 2-[18F]fluoro-2-deoxy-D-glucose. Int J Radiat Oncol Biol Phys 2004;59:1295-1300.

17. Borst GR, Belderbos JS, Boellaard R, et al. Standardised FDG uptake: a prognostic factor for inoperable non-small cell lung cancer. Eur J Cancer 2005;41:1533-1541.

18. Brun E, Kjellen E, Tennvall J, et al. FDG PET studies during treatment: prediction of therapy outcome in head and neck squamous cell carcinoma. Head Neck 2002;24:127-135.

19. Dhital K, Saunders CA, Seed PT, et al. [(18)F]Fluorodeoxyglucose positron emission tomography and its prognostic value in lung cancer. Eur J Cardiothorac Surg 2000;18:425-428.

20. Downey RJ, Akhurst T, Gonen M, et al. Preoperative F-18 fluorodeoxyglucose-positron emission tomography maximal standardized uptake value predicts survival after lung cancer resection. J Clin Oncol 2004;22:3255-3260.

21. Higashi K, Ueda $Y$, Arisaka $Y$, et al. 18F-FDG uptake as a biologic prognostic factor for recurrence in patients with surgically resected non-small cell lung cancer. J Nucl Med 2002;43:3945. 
22. Jeong HJ, Min JJ, Park JM, et al. Determination of the prognostic value of [(18)F] fluorodeoxyglucose uptake by using positron emission tomography in patients with non-small cell lung cancer. Nucl Med Commun 2002;23:865-870.

23. Vansteenkiste JF, Stroobants SG, Dupont PJ, et al. Prognostic importance of the standardized uptake value on (18)F-fluoro-2-deoxy-glucose-positron emission tomography scan in nonsmall-cell lung cancer: An analysis of 125 cases. Leuven Lung Cancer Group. J Clin Oncol 1999;17:3201-3206.

24. Sasaki R, Komaki R, Macapinlac $H$, et al. [18F]fluorodeoxyglucose uptake by positron emission tomography predicts outcome of non-small-cell lung cancer. J Clin Oncol 2005;23:1136-1143.

25. Erdi YE, Macapinlac $\mathrm{H}$, Rosenzweig KE, et al. Use of PET to monitor the response of lung cancer to radiation treatment. Eur J Nucl Med 2000;27:861-866.

26. Kitagawa Y, Nishizawa S, Sano K, et al. Prospective comparison of 18F-FDG PET with conventional imaging modalities (MRI, CT, and 67Ga scintigraphy) in assessment of combined intraarterial chemotherapy and radiotherapy for head and neck carcinoma. J Nucl Med 2003;44:198206.

27. Koike I, Ohmura M, Hata M, et al. FDG-PET scanning after radiation can predict tumor regrowth three months later. Int J Radiat Oncol Biol Phys 2003;57:1231-1238.

28. Kunkel M, Forster GJ, Reichert $T E$, et al. Radiation response non-invasively imaged by [18F]FDG-PET predicts local tumor control and survival in advanced oral squamous cell carcinoma. Oral Oncol 2003;39:170-177.

29. Mac Manus MP, Hicks RJ, Matthews JP, et al. Positron emission tomography is superior to computed tomography scanning for response-assessment after radical radiotherapy or chemoradiotherapy in patients with non-small-cell lung cancer. J Clin Oncol 2003;21:1285-1292.

30. Porceddu SV, Jarmolowski E, Hicks RJ, et al. Utility of positron emission tomography for the detection of disease in residual neck nodes after (chemo)radiotherapy in head and neck cancer. Head Neck 2005;27:175-181.

31. Rogers JW, Greven KM, McGuirt WF, et al. Can post-RT neck dissection be omitted for patients with head-and-neck cancer who have a negative PET scan after definitive radiation therapy? Int J Radiat Oncol Biol Phys 2004;58:694-697.

32. Yao M, Graham MM, Hoffman HT, et al. The role of post-radiation therapy FDG PET in prediction of necessity for post-radiation therapy neck dissection in locally advanced head-and-neck squamous cell carcinoma. Int J Radiat Oncol Biol Phys 2004;59:1001-1010.

33. Brink I, Schumacher T, Mix M, et al. Impact of [18F]FDG-PET on the primary staging of smallcell lung cancer. Eur J Nucl Med Mol Imaging 2004;31:1614-1620.

34. Mah K, Caldwell CB, Ung YC, et al. The impact of (18)FDG-PET on target and critical organs in CT-based treatment planning of patients with poorly defined non-small-cell lung carcinoma: a prospective study. Int J Radiat Oncol Biol Phys 2002;52:339-350.

35. Ciernik IF, Dizendorf E, Baumert BG, et al. Radiation treatment planning with an integrated positron emission and computer tomography (PET/CT): a feasibility study. Int J Radiat Oncol Biol Phys 2003;57:853-863.

36. Paulsen F, Scheiderbauer J, Eschmann SM, et al. First experiences of radiation treatment planning with PET/CT. Strahlenther Onkol 2006;182:369-375.

37. Dizendorf EV, Baumert BG, von Schulthess GK, et al. Impact of whole-body 18F-FDG PET on staging and managing patients for radiation therapy. J Nucl Med 2003;44:24-29.

38. Kalff V, Hicks RJ, MacManus MP, et al. Clinical impact of (18)F fluorodeoxyglucose positron emission tomography in patients with non-small-cell lung cancer: a prospective study. J Clin Oncol 2001;19:111-118.

39. Macmanus M, D'Costa I, Everitt S, et al. Comparison of CT and positron emission tomography/CT coregistered images in planning radical radiotherapy in patients with non-small-cell lung cancer. Australas Radiol 2007;51:386-393.

40. Grills IS, Yan D, Black QC, et al. Clinical implications of defining the gross tumor volume with combination of CT and 18FDG-positron emission tomography in non-small-cell lung cancer. Int J Radiat Oncol Biol Phys 2007;67:709-719.

41. Bujenovic S. The role of positron emission tomography in radiation treatment planning. Semin Nucl Med 2004;34:293-299. 
42. Caldwell CB, Mah K, Ung YC, et al. Observer variation in contouring gross tumor volume in patients with poorly defined non-small-cell lung tumors on CT: the impact of 18FDG-hybrid PET fusion. Int J Radiat Oncol Biol Phys 2001;51:923-931.

43. Steenbakkers RJ, Duppen JC, Fitton I, et al. Reduction of observer variation using matched CTPET for lung cancer delineation: a three-dimensional analysis. Int $J$ Radiat Oncol Biol Phys 2006;64:435-448.

44. Brianzoni E, Rossi G, Ancidei S, et al. Radiotherapy planning: PET/CT scanner performances in the definition of gross tumour volume and clinical target volume. Eur J Nucl Med Mol Imaging 2005;32:1392-1399.

45. Ceresoli GL, Cattaneo GM, Castellone P, et al. Role of computed tomography and [18F] fluorodeoxyglucose positron emission tomography image fusion in conformal radiotherapy of nonsmall cell lung cancer: a comparison with standard techniques with and without elective nodal irradiation. Tumori 2007;93:88-96.

46. Deniaud-Alexandre E, Touboul E, Lerouge D, et al. Impact of computed tomography and 18Fdeoxyglucose coincidence detection emission tomography image fusion for optimization of conformal radiotherapy in non-small-cell lung cancer. Int J Radiat Oncol Biol Phys 2005;63:14321441.

47. Ashamalla H, Rafla S, Parikh K, et al. The contribution of integrated PET/CT to the evolving definition of treatment volumes in radiation treatment planning in lung cancer. Int J Radiat Oncol Biol Phys 2005;63:1016-1023.

48. Bradley J, Thorstad WL, Mutic S, et al. Impact of FDG-PET on radiation therapy volume delineation in non-small-cell lung cancer. Int J Radiat Oncol Biol Phys 2004;59:78-86.

49. Nestle U, Walter K, Schmidt S, et al. 18F-deoxyglucose positron emission tomography (FDGPET) for the planning of radiotherapy in lung cancer: high impact in patients with atelectasis. Int J Radiat Oncol Biol Phys 1999;44:593-597.

50. Erdi YE, Rosenzweig K, Erdi AK, et al. Radiotherapy treatment planning for patients with nonsmall cell lung cancer using positron emission tomography (PET). Radiother Oncol 2002;62:5160.

51. Munley MT, Marks LB, Scarfone C, et al. Multimodality nuclear medicine imaging in threedimensional radiation treatment planning for lung cancer: challenges and prospects. Lung Cancer 1999;23:105-114.

52. Tralins KS, Douglas JG, Stelzer KJ, et al. Volumetric analysis of 18F-FDG PET in glioblastoma multiforme: prognostic information and possible role in definition of target volumes in radiation dose escalation. J Nucl Med 2002;43:1667-1673.

53. Bentzen SM. Theragnostic imaging for radiation oncology: dose-painting by numbers. Lancet Oncol 2005;6:112-117.

54. Dwamena BA, Sonnad SS, Angobaldo JO, et al. Metastases from non-small cell lung cancer: mediastinal staging in the 1990s--meta-analytic comparison of PET and CT. Radiology $1999 ; 213: 530-536$.

55. Fischer BM, Mortensen J, Hojgaard L. Positron emission tomography in the diagnosis and staging of lung cancer: a systematic, quantitative review. Lancet Oncol 2001;2:659-666.

56. Kramer $\mathrm{H}$, Groen $\mathrm{HJ}$. Current concepts in the mediastinal lymph node staging of nonsmall cell lung cancer. Ann Surg 2003;238:180-188.

57. Gould MK, Kuschner WG, Rydzak CE, et al. Test performance of positron emission tomography and computed tomography for mediastinal staging in patients with non-small-cell lung cancer: a meta-analysis. Ann Intern Med 2003;139:879-892.

58. Gregoire V. Is there any future in radiotherapy planning without the use of PET: unraveling the myth. Radiother Oncol 2004;73:261-263.

59. Daisne JF, Duprez T, Weynand B, et al. Tumor volume in pharyngolaryngeal squamous cell carcinoma: comparison at CT, MR imaging, and FDG PET and validation with surgical specimen. Radiology 2004;233:93-100.

60. Geets X, Lee JA, Bol A, et al. A gradient-based method for segmenting FDG-PET images: methodology and validation. Eur J Nucl Med Mol Imaging 2007. 
61. Court LE, Dong L, Taylor N, et al. Evaluation of a contour-alignment technique for CT-guided prostate radiotherapy: an intra- and interobserver study. Int $\mathrm{J}$ Radiat Oncol Biol Phys 2004;59:412-418.

62. Valicenti RK, Sweet JW, Hauck WW, et al. Variation of clinical target volume definition in threedimensional conformal radiation therapy for prostate cancer. Int $\mathrm{J}$ Radiat Oncol Biol Phys 1999;44:931-935.

63. Logue JP, Sharrock CL, Cowan RA, et al. Clinical variability of target volume description in conformal radiotherapy planning. Int J Radiat Oncol Biol Phys 1998;41:929-931.

64. Weltens C, Menten J, Feron M, et al. Interobserver variations in gross tumor volume delineation of brain tumors on computed tomography and impact of magnetic resonance imaging. Radiother Oncol 2001;60:49-59.

65. Fox JL, Rengan R, O'Meara W, et al. Does registration of PET and planning CT images decrease interobserver and intraobserver variation in delineating tumor volumes for non-small-cell lung cancer? Int J Radiat Oncol Biol Phys 2005;62:70-75.

66. Black QC, Grills IS, Kestin LL, et al. Defining a radiotherapy target with positron emission tomography. Int J Radiat Oncol Biol Phys 2004;60:1272-1282.

67. Bradley JD, Dehdashti F, Mintun MA, et al. Positron emission tomography in limited-stage small-cell lung cancer: a prospective study. J Clin Oncol 2004;22:3248-3254.

68. Ciernik IF, Huser M, Burger $C$, et al. Automated functional image-guided radiation treatment planning for rectal cancer. Int J Radiat Oncol Biol Phys 2005;62:893-900.

69. Daisne JF, Sibomana M, Bol A, et al. Tri-dimensional automatic segmentation of PET volumes based on measured source-to-background ratios: influence of reconstruction algorithms. Radiother Oncol 2003;69:247-250.

70. Erdi YE, Mawlawi O, Larson SM, et al. Segmentation of lung lesion volume by adaptive positron emission tomography image thresholding. Cancer 1997;80:2505-2509.

71. Paulino AC, Johnstone PA. FDG-PET in radiotherapy treatment planning: Pandora's box? Int J Radiat Oncol Biol Phys 2004;59:4-5.

72. Nestle U, Kremp S, Schaefer-Schuler A, et al. Comparison of different methods for delineation of 18 F-FDG PET-positive tissue for target volume definition in radiotherapy of patients with nonSmall cell lung cancer. J Nucl Med 2005;46:1342-1348.

73. Thie JA. Understanding the standardized uptake value, its methods, and implications for usage. J Nucl Med 2004;45:1431-1434.

74. Boellaard R, Krak NC, Hoekstra OS, et al. Effects of noise, image resolution, and ROI definition on the accuracy of standard uptake values: a simulation study. J Nucl Med 2004;45:1519-1527.

75. Erdi YE, Nehmeh SA, Pan T, et al. The CT motion quantitation of lung lesions and its impact on PET-measured SUVs. J Nucl Med 2004;45:1287-1292.

76. Hoekstra CJ, Paglianiti I, Hoekstra OS, et al. Monitoring response to therapy in cancer using [18F]-2-fluoro-2-deoxy-D-glucose and positron emission tomography: an overview of different analytical methods. Eur J Nucl Med 2000;27:731-743.

77. Vansteenkiste JF. Nodules, CT-scans and PET-scans: a good partnership. Lung Cancer 2004;45:29-30.

78. Vanuytsel L, Vansteenkiste JF, Stroobants SG, et al. The impact of (18)F-fluoro-2-deoxy-Dglucose positron emission tomography (FDG-PET) lymph node staging on the radiation treatment volumes in patients with non-small cell lung cancer. Radiother Oncol 2000;55:317-324.

79. Birim O, Kappetein AP, Stijnen T, et al. Meta-analysis of positron emission tomographic and computed tomographic imaging in detecting mediastinal lymph node metastases in nonsmall cell lung cancer. Ann Thorac Surg 2005;79:375-382.

80. Kiffer JD, Berlangieri SU, Scott AM, et al. The contribution of 18F-fluoro-2-deoxy-glucose positron emission tomographic imaging to radiotherapy planning in lung cancer. Lung Cancer 1998;19:167-177.

81. Giraud P, Grahek D, Montravers F, et al. CT and (18)F-deoxyglucose (FDG) image fusion for optimization of conformal radiotherapy of lung cancers. Int $J$ Radiat Oncol Biol Phys 2001;49:1249-1257. 
82. Nestle U, Hellwig D, Schmidt S, et al. 2-Deoxy-2-[18F]fluoro-D-glucose positron emission tomography in target volume definition for radiotherapy of patients with non-small-cell lung cancer. Mol Imaging Biol 2002;4:257-263.

83. Senan S, Burgers S, Samson MJ, et al. Can elective nodal irradiation be omitted in stage III non-small-cell lung cancer? Analysis of recurrences in a phase II study of induction chemotherapy and involved-field radiotherapy. Int J Radiat Oncol Biol Phys 2002;54:999-1006.

84. Sorensen $P$ HO. The location of recurrences in patients with non-small lung cancer treated with involved-field irradiation [Abstract]. Lung Cancer; 2000. p. S171.

85. van Der Wel A, Nijsten S, Hochstenbag M, et al. Increased therapeutic ratio by 18FDG-PET CT planning in patients with clinical CT stage N2-N3M0 non-small-cell lung cancer: a modeling study. Int J Radiat Oncol Biol Phys 2005;61:649-655.

86. De Ruysscher D, Wanders S, van Haren E, et al. Selective mediastinal node irradiation based on FDG-PET scan data in patients with non-small-cell lung cancer: a prospective clinical study. Int J Radiat Oncol Biol Phys 2005;62:988-994.

87. Belderbos JS, Heemsbergen WD, De Jaeger K, et al. Final results of a Phase I/II dose escalation trial in non-small-cell lung cancer using three-dimensional conformal radiotherapy. Int J Radiat Oncol Biol Phys 2006;66:126-134.

88. Giraud $\mathrm{P}$, Antoine $\mathrm{M}$, Larrouy $\mathrm{A}$, et al. Evaluation of microscopic tumor extension in non-smallcell lung cancer for three-dimensional conformal radiotherapy planning. Int $J$ Radiat Oncol Biol Phys 2000;48:1015-1024.

89. Grills IS, Fitch DL, Goldstein NS, et al. Clinicopathologic Analysis of Microscopic Extension in Lung Adenocarcinoma: Defining Clinical Target Volume for Radiotherapy. Int J Radiat Oncol Biol Phys 2007.

90. Yuan S, Meng X, Yu J, et al. Determining optimal clinical target volume margins on the basis of microscopic extracapsular extension of metastatic nodes in patients with non-small-cell lung cancer. Int J Radiat Oncol Biol Phys 2007;67:727-734.

91. De Ruysscher D, Wanders S, Minken A, et al. Effects of radiotherapy planning with a dedicated combined PET-CT-simulator of patients with non-small cell lung cancer on dose limiting normal tissues and radiation dose-escalation: A planning study. Radiother Oncol 2005;77:5-10.

92. Biehl KJ, Kong FM, Dehdashti F, et al. 18F-FDG PET definition of gross tumor volume for radiotherapy of non-small cell lung cancer: is a single standardized uptake value threshold approach appropriate? J Nucl Med 2006;47:1808-1812.

93. Nestle U, Schaefer-Schuler A, Kremp S, et al. Target volume definition for 18F-FDG PETpositive lymph nodes in radiotherapy of patients with non-small cell lung cancer. Eur J Nucl Med Mol Imaging 2007;34:453-462. 


-

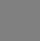

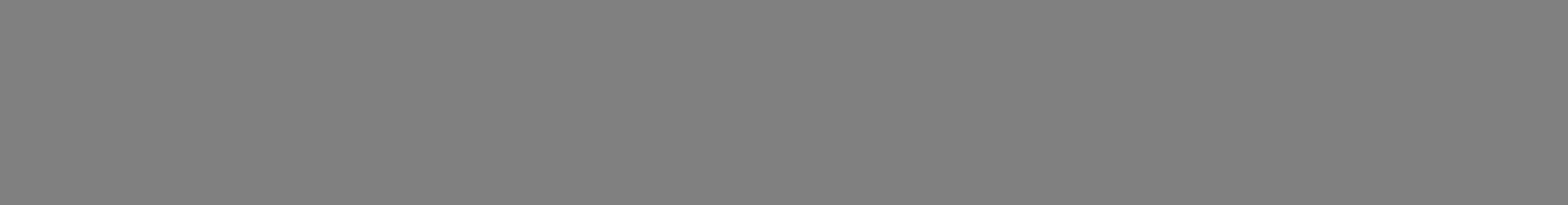

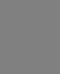

(1)





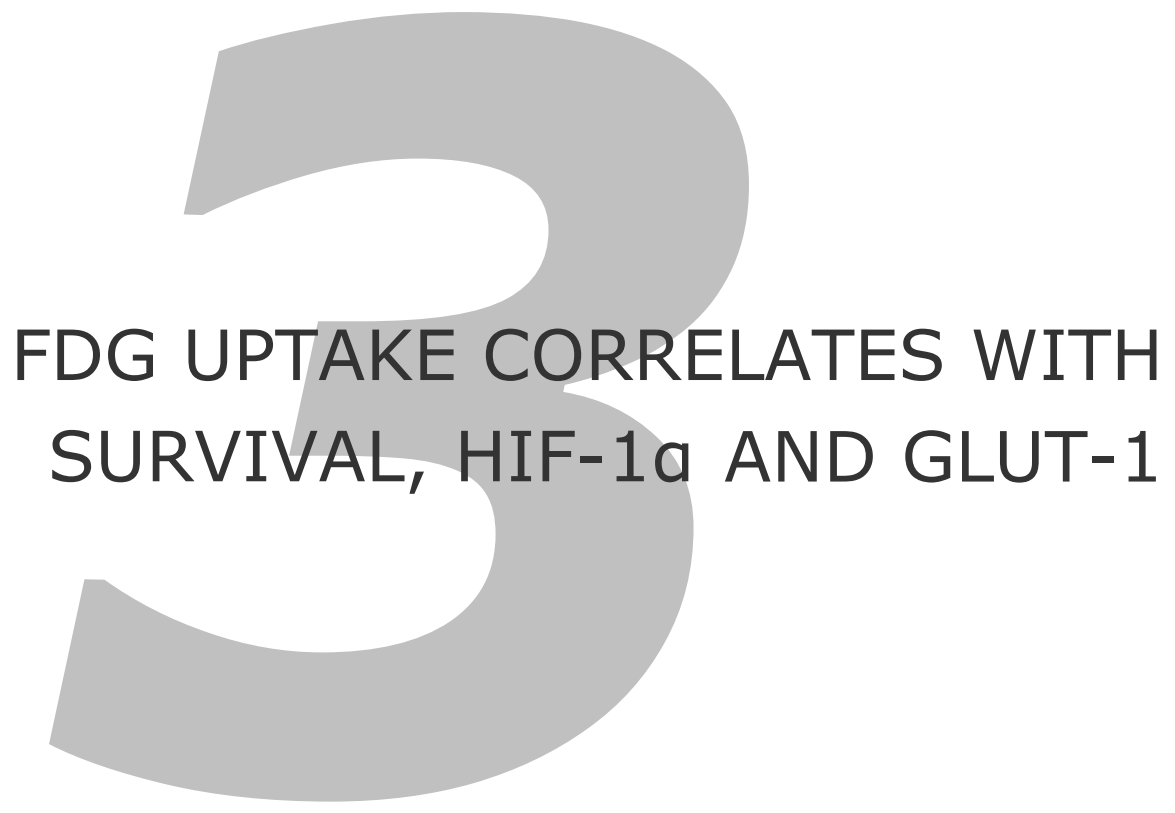

A. van Baardwijk*, C. Dooms*, R.J. van Suylen, E. Verbeken, M. Hochstenbag, C. Dehing-Oberije, D. Rupa, S. Pastorekova, S. Stroobants, U. Buell, P. Lambin, J. Vansteenkiste and D. De Ruysscher. The maximum uptake of ${ }^{18} \mathrm{~F}$-deoxyglucose on PET scan correlates with survival, Hypoxia Inducible Factor-1a and GLUT-1 in Non-Small Cell Lung Cancer. (*these authors equally contributed to this article)

Eur J Cancer 2007;43(9):1392-8 


\section{Abstract}

Introduction: The purpose of this study was to investigate the relation between the standardized uptake value (SUV) on FDG-PET scan and hypoxia related markers (HIF-1a and CAIX), a proliferation-related marker (Ki-67) and glucose transporters (GLUT-1 and GLUT-3) in non-small cell lung cancer (NSCLC).

Materials and Methods: One hundred and two patients, scheduled for complete resection, received a PET scan in Leuven or Maastricht/Aachen. The maximal SUV $\left(\mathrm{SUV}_{\max }\right)$ was correlated with survival and immunohistochemical staining patterns.

Results: The actuarial survival was worse for patients showing a high SUV max $_{\text {, the best }}$ discriminative value being 8.0 (Leuven, $p=0.032$ ) and 11.0 (Maastricht, $p=0.007$ ). Tumours with a high SUV max $_{\text {max }}$ expressed in a higher proportion HIF-1a $(63.1 \%$ versus $37.9 \%, p=0.024)$ and GLUT-1 (82.8\% versus $62.5 \%, p=0.025)$, than tumours with a low SUV $\mathrm{V}_{\max }$. No significant difference was found in the expression of CAIX, Ki-67 and GLUT-3.

Conclusions: This study supports preclinical data that hypoxia is associated with a higher uptake of FDG. 


\section{Introduction}

TNM-stage is up till now the best prognostic indicator for survival after radical operation in NSCLC ${ }^{1}$. However, even patients with the same stage may have a very different survival. Pre-treatment characteristics that add prognostic information are therefore of interest. Non-invasive imaging modalities, like positron emission tomography (PET), are increasingly used in the staging and treatment of NSCLC. The maximal uptake of ${ }^{18} \mathrm{~F}$-fluoro-2-deoxy-glucose (FDG) in the primary tumour was consistently shown to be an independent prognostic factor for survival ${ }^{2-5}$. However, the actual mechanisms by which a high FDG uptake leads to a worse prognosis are not well known. Different molecular markers, representing independent pathways, like hypoxia, apoptosis and angiogenesis, have been associated with a high risk of recurrence and death in NSCLC patients ${ }^{6-8}$.

Hypoxia might play an important role in the uptake of FDG, since it leads via the Hypoxia Inducible Factor-1a (HIF-1a) pathway to up regulation of glucose transporters, Carbonic Anhydrase IX (CAIX) and other target genes ${ }^{9,10}$. Preclinical studies suggest that hypoxic conditions correspond to a higher FDG uptake ${ }^{11-14}$. In addition hypoxia is an important cause of treatment failure in many tumours and both HIF-1a and CAIX have been related to a poor outcome $e^{6,8,15,16}$.

Since hypoxia leads to an increased rate of glycolysis, which in turn, increases the uptake of FDG, we hypothesized that the worse prognosis of NSCLC patients with a high FDG uptake would be related to hypoxia. Therefore we investigated the impact of tumour hypoxia, as assessed by the expression of the endogenous hypoxia markers HIF-1a and CAIX, and glucose metabolism (GLUT-1 and GLUT-3) on the uptake of FDG on PET scan before surgery in NSCLC.

\section{Materials and Methods}

\section{Study population}

The surgical specimens evaluated were taken from 102 patients with a histological proven NSCLC. All patients with a clinical stage I or II, who had undergone resection of their tumour with curative intent and had had a diagnostic PET scan, were included. All patients were operated on at the University Hospital Gasthuisberg of Leuven $(n=56$, November 1994 - October 1997) or the University Hospital of Maastricht $(n=46$, February 1998 - September 2002). Only adenocarcinoma, squamous cell carcinoma and large cell/undifferentiated carcinoma were included. FDG-PET scanning was performed on two different types of PET-scanners. All patients fasted for at least 6 hours before scanning and glucose levels were checked.

The first group of patients from Leuven $(n=56)$ was scanned with a CTI-Siemens (Iselin, NJ) 931/08/12 PET scanner with an axial field of view (FOV) of $10.1 \mathrm{~cm}$ and a spatial resolution of $8 \mathrm{~mm}$. After injection $(6.5 \mathrm{MBq} / \mathrm{kg}$, maximal $555 \mathrm{MBq}$ ) a $60 \mathrm{~min}$ dynamic emission study was followed by a $10 \mathrm{~min}$ static acquisition. Images were reconstructed using filtered back projection. The patients from Maastricht $(n=46)$ were 
scanned with an ECAT EXACT 922 (Siemens-CTI, Knoxville, TN) in Aachen with an axial FOV of $162 \mathrm{~mm}$ and a spatial resolution of $6 \mathrm{~mm}$. After a median time of $60 \mathrm{~min}$ (range 45-120 min) after injection (3.5 MBq/ $\mathrm{kg}$ ) 2-D whole body emission images were acquired. Images were reconstructed using an iterative reconstruction algorithm. For the determination of the Standardized Uptake Value (SUV) a Region of Interest (ROI) was drawn by the nuclear medicine physician on the transaxial images around the primary tumour. SUV was then automatically calculated as activity concentration of FDG uptake divided by injected dose/body weight ${ }^{17}$. To avoid partial volume effects as much as possible, the maximal SUV (SUV $\max$ ) within this ROI was calculated. Since the 2 PET-scanners used in this study have different characteristics, a potential concern was different measurements of SUV $\max$ on the two machines. Phantom measurements to compare the 2 machines could not be performed, since the CTI-Siemens was no longer used. Since direct comparison of the results of the 2 groups was not possible, we determined the best discriminating factor for survival for both groups scanned on the 2 separate PET scanners, as has been described by Vansteenkiste and colleagues and used by other authors ${ }^{4,5}$. Different cut-off levels, within an in literature most often mentioned range between 5 and 13, were used for this purpose.

The final staging was based on the findings at pathologic examination (TNM classification, $6^{\text {th }}$ edition, 2002). Follow-up data from all patients were collected until August 2005, using the patient's files. If necessary, the patient's general practitioner or referring specialist was contacted to complete follow-up. Since data were collected retrospectively, only overall survival was estimated.

\section{Materials}

The specimens studied were routinely processed, formalin-fixed and paraffinembedded. Representative histological sections of the tumour specimen were taken to cut tissue sections of $4 \mu \mathrm{m}$ thicknesses and stained with haematoxylin and eosin. For immunohistochemical staining sections were dewaxed in xylene and rehydrated by passage through graded alcohols. Endogenous peroxidase was blocked by applying $0.03 \%$ hydrogen peroxidase $(20 \mathrm{~min})$. If necessary antigen retrieval was achieved using a citrate or TE buffer and sections were pretreated with blocking normal rabbit (CAIX) or calf (HIF-1a) serum. The following primary antibodies were used: mouse monoclonal antihuman CAIX antibody M75 (Slovak Academy of Sciences, Bratislava, $1: 50,45 \mathrm{~min}$ ), mouse monoclonal antihuman HIF-1a antibody (BD Biosciences Pharmingen, 1:50, overnight), rabbit anti-GLUT-1 and anti-GLUT-3, (AB1341 and AB1345 polyclonal antiserum, Chemicon, Temecula, 1:500, 2 hrs) and monoclonal mouse antihuman MIB-1 antibody (Ki-67 antigen DAKO M 7240, 1:100, $45 \mathrm{~min}$ ).

As second layer Dako Envision+ (CAIX and HIF-1a) or biotinylated antibody and biotinylated horse-radish peroxidase complex (GLUT-1, GLUT-3 and Ki-67) were used. Finally all slides were developed with diaminobenzidine (DAB) and counterstained with hematoxylin, dehydrated and mounted. Positive and negative (primary antibody omitted) controls were used to check the procedures.

Sections were assessed using light microscope in a blinded fashion by at least two observers ( $A v B, D R$ and RJvS). If discrepancies were found, a consensus was reached using a conference microscope. For Ki-67 at least 500 tumour cells were counted and the percentage of $\mathrm{Ki}-67$ positive cells was noted. Tissue samples showing no or just 
weak membranous staining for GLUT-1 and GLUT-3 were considered negative, whereas strong membranous staining was considered positive. For CAIX membranous (m)CAIX staining and nuclear staining of HIF-1a a semi-quantitive scoring method was used: $0=<5 \%, 1=5-25 \%, 2=25-50 \%, 3=50-75 \%$ and $4=>75 \%$ of cells positive. Finally the results were dichotomized. Tumours with no or low positive staining were considered negative and tumours with $>25 \%$ positivity were considered positive.

\section{Statistics}

The SPSS software (version 14.0, SPSS Inc., Chicago, IL) was used to perform statistical analysis. Results are expressed as mean \pm standard deviation (S.D.) and range, unless otherwise indicated. Survival curves were analysed using the Kaplan-Meier method and the log-rank test. The Chi-square test, Pearson correlation coefficient and the Mann Whitney U-test were used to analyse the association between the different categorical variables. P-values less than 0.05 were considered statistically significant.

\section{Ethics}

According to Dutch and Belgian law and regulations of the Medical Ethical Committees, no informed consent was required for this study.

\section{Results}

\section{Patient and tumour characteristics}

The patient and tumour characteristics are depicted in Table 3.1. In total 102 patients, 83 males and 19 females were included in the study. The mean follow up was 42 months (range 1-96 months) and the 2-year actuarial survival was $68.6 \%$. One patient $(1.0 \%)$ died within 30 days postoperatively due to cardial problems. Most tumours were squamous cell carcinoma $(n=58,56.9 \%)$, while $30.4 \% \quad(n=31)$ consisted of adenocarcinoma and $12.7 \%(n=13)$ of large cell carcinomas.

\begin{tabular}{|llcc|}
\hline Table 3.1 Overview of patient characteristics and tumour characteristics. & \\
\hline Characteristics & & & $(37-85)$ \\
Mean age (years) & Male & 64.3 & $(81.4)$ \\
Gender & Female & 83 & $(18.6)$ \\
& Adenocarcinoma & 19 & $(30.4)$ \\
Pathology & Squamous cell carcinoma & 31 & $(56.9)$ \\
& Large cell carcinoma & 58 & $(12.7)$ \\
& IA & 13 & $(25.5)$ \\
Pathologic staging group & 26 & $(39.2)$ \\
& IB & 40 & $(0)$ \\
& IIA & 0 & $(23.5)$ \\
& IIB & 24 & $(9.8)$ \\
& IIIB & 10 & $(2.0)$ \\
\hline
\end{tabular}




\section{PET data and survival}

For the first group of patients from Leuven a median $\mathrm{SUV}_{\max }$ of 10.0 (mean 10.8, S.D. 5.2, range 1.7-25.4) was observed. The most discriminative cut-off point for survival was found at SUV $\max$ of 8.0 (Figure 3.1a). A statistically significant worse survival was noted in patients having a tumour with a $S U V_{\max } \geq 8$, compared with patients showing a tumour with a $\mathrm{SUV}_{\max }<8$, a 2-year survival of $56.8 \%$ and $89.5 \%$ respectively $(p=0.032$; Figure $3.2 a)$. For the second group of patients from Maastricht, a median SUV $V_{\max }$ of 12.7 (mean 14.1, S.D. 6.5, range 4.2-31.5) was found. The best discriminative cut-off point for this group was observed at a SUV $\max$ of 11.0 (Figure 3.1b). Patients with a tumour with a $S_{U} V_{\max }>11$ showed a 2-year survival of $60.6 \%$, while patients with a tumour showing a $S_{U} V_{\max }<11$ showed a 2 - year survival of $92.3 \%$ $(p=0.007$; Figure 3.2b). The 2 groups of the different PET scanners showing a high uptake of FDG were combined and referred to as high SUV $_{\max }$ group further on. The combination of the groups with a SUV $<8$ and $<11$ respectively referred to as low SUV $_{\max }$. The combining of the groups showed a 2 -year survival of $90.6 \%$ for the patients with a low SUV $\max$ and $58.6 \%$ for the patients with a high SUV $\max (p=0.001)$.
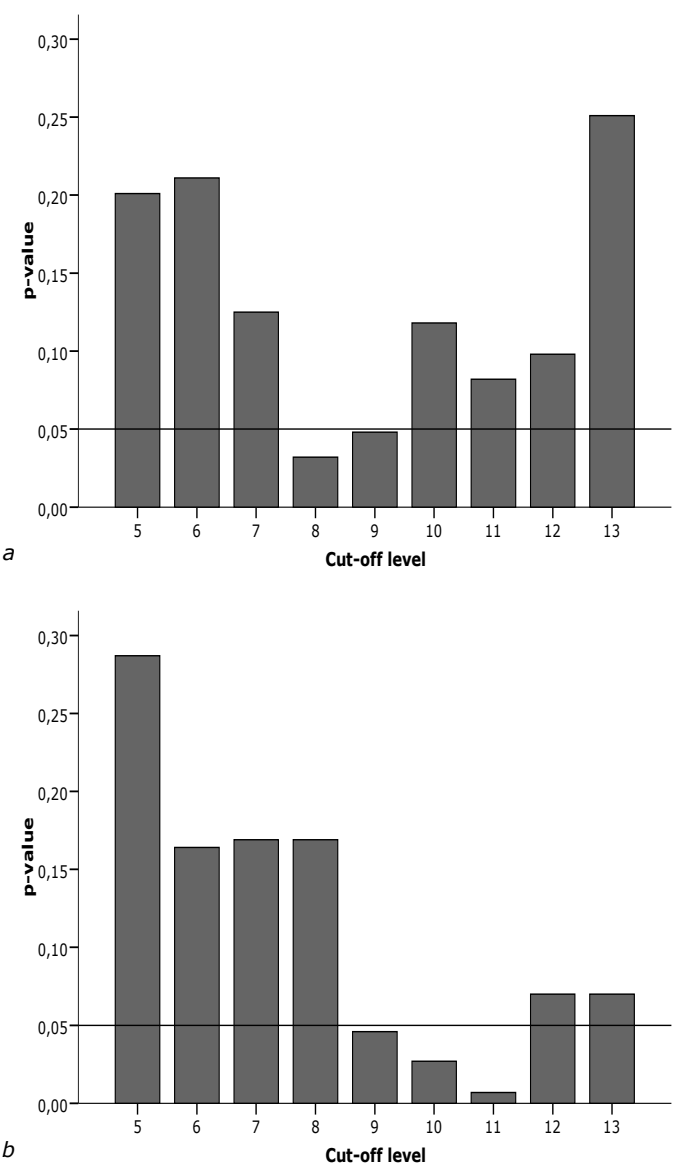

Figure 3.1 P-values of log rank-test for actuarial survival using different cut-off levels for SUVmax for the group of patients from Leuven (a) and from Maastricht $b$ ). 

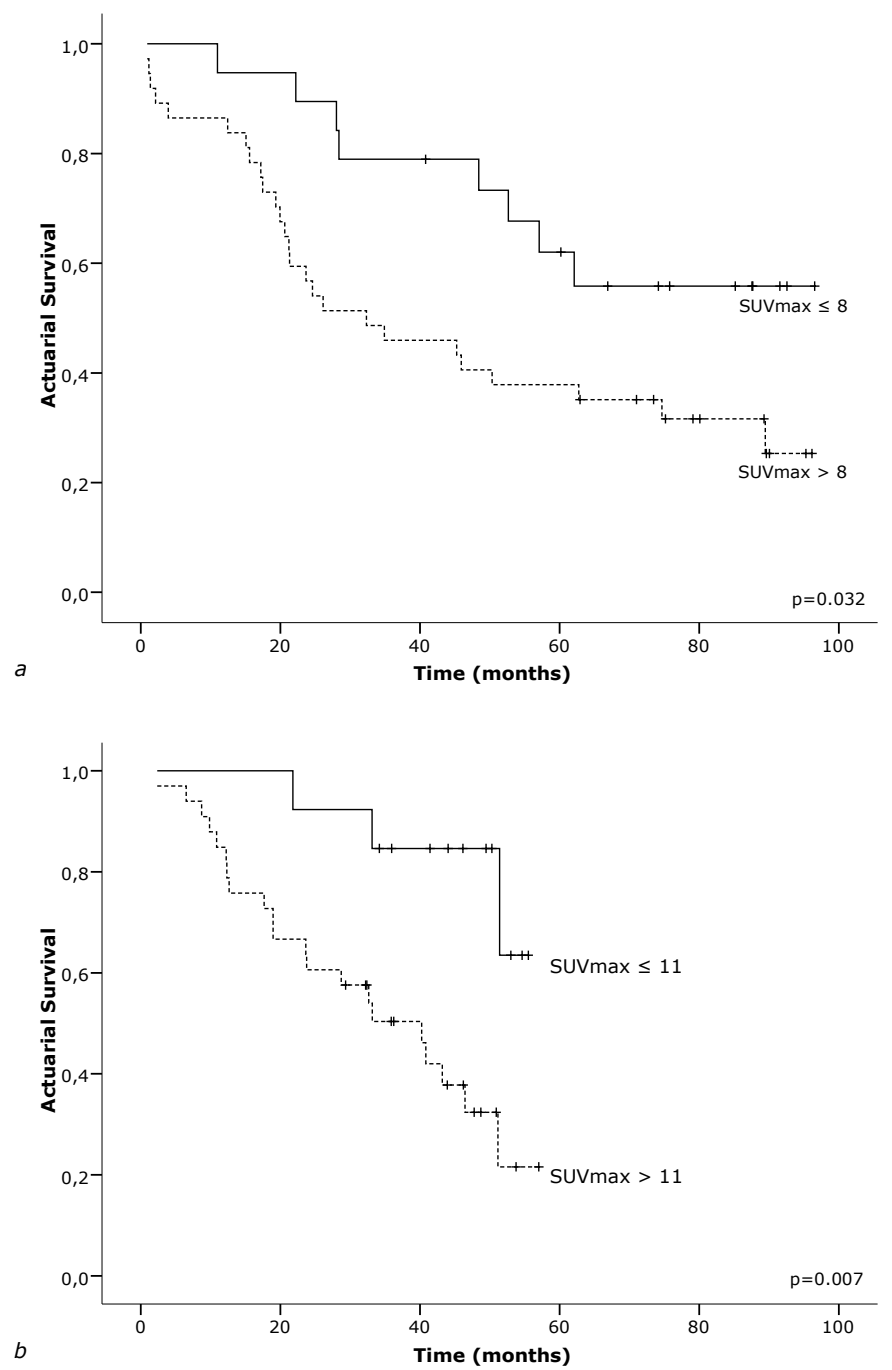

Figure 3.2 Kaplan-Meier survival curve for actuarial survival for maximal SUV (tumours with a high SUV $_{\max }$ versus tumours with a low SUV $V_{\max }$ ) applying a threshold for SUV $V_{\max }$ of 8.0 for Leuven (a) and a SUV $V_{\max }$ of 11.0 for Maastricht (b).

\section{PET data and immunohistochemical staining}

All immunohistochemical staining results were associated with SUV $V_{\max }$ levels above and below the above-mentioned cut off points. Figure 3.3 shows the different IHC staining patterns for the markers. In total, $55 \%$ of the tumours showed positive nuclear staining of HIF-1a. Tumour samples with a high SUV max $_{\text {max }}$ show positivity for HIF-1a in $63.1 \%$, while only $37.9 \%$ of the samples with a low SUV $\max$ showed nuclear staining $(p=0.024)$. Of all samples positive for HIF-1a $57 \%$ were positive for CAIX, whereas $77 \%$ of cases being positive for CAIX showed nuclear staining for HIF-1a. Although a correlation between HIF-1a and CAIX staining was observed (correlation coefficient $0.363, p=0.001)$, no significant difference in CAIX staining was observed between the 


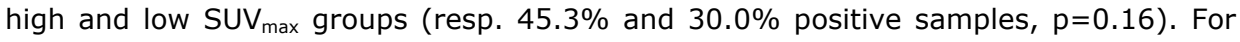
the proliferation-associated marker $\mathrm{Ki}-67$, the mean percentage of tumour cells being positive was $42.7 \%$ (S.D. 21.7 , range $2.7-95.2 \%$ ). In tumours with a high uptake of FDG $44.4 \%$ of cell were positive for Ki-67, while tumours with a low SUV $V_{\max }$ showed a mean percentage $39.1 \%(p=0.20)$. For the glucose transporters, in total $76.5 \%$ of the tumours stained positive for GLUT- 1 and $44.1 \%$ for GLUT-3. The tumours with a high SUV $_{\max }$ showed in $82.9 \%$ of cases membranous staining of GLUT-1, whereas tumours with a low uptake only in $62.5 \%(p=0.025)$. For GLUT-3 there was no statistically significant difference $(p=0.21)$. The association between $S V_{\max }$ and the expression of the different markers and the correlation coefficients of the markers are depicted in Table 3.2 and Table 3.3.
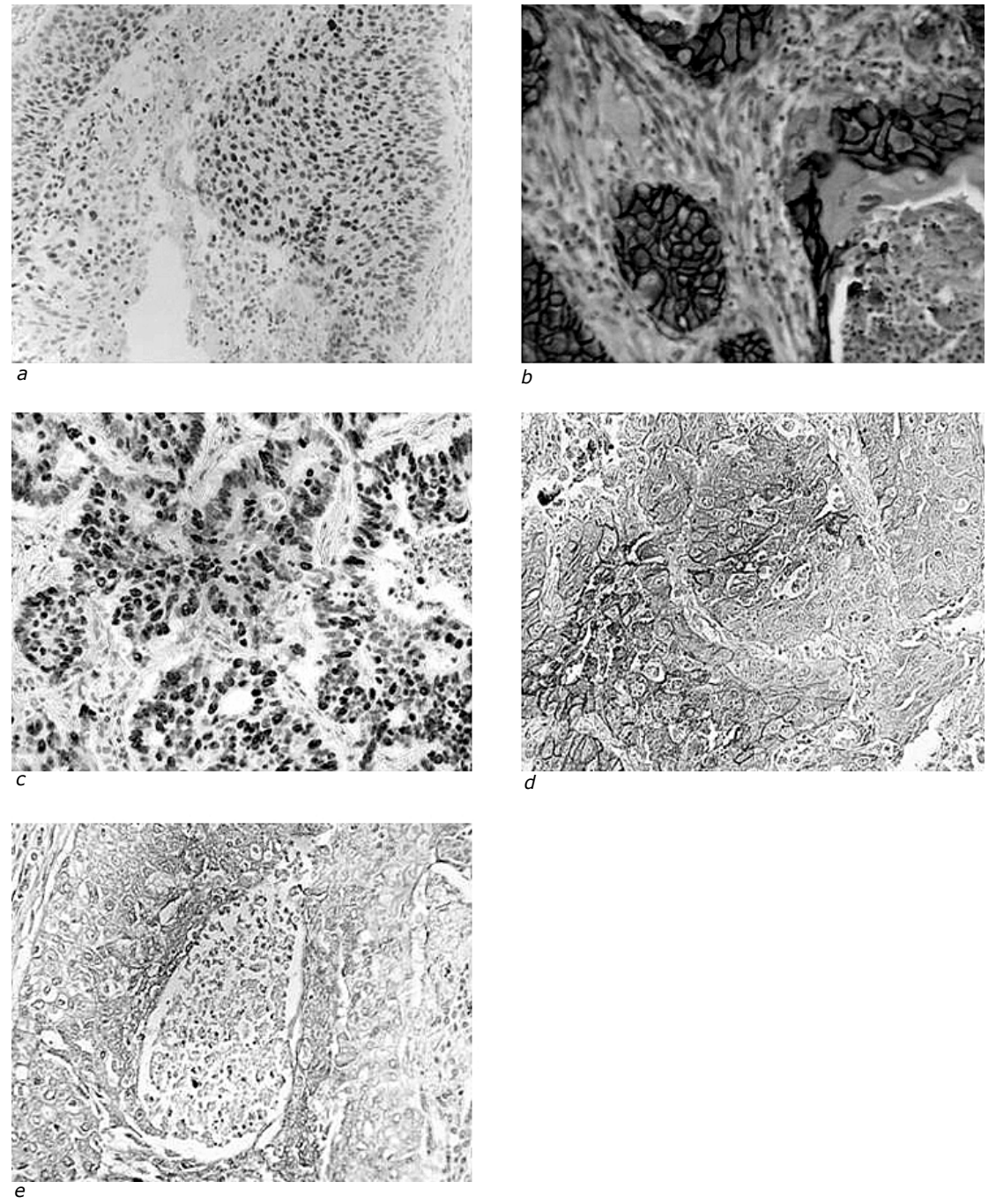

Figure 3.3 Immunohistochemical staining. Positive nuclear staining for HIF-1a (a), positive membranous staining for CAIX (b), nuclear staining of Ki-67 (c), and membranous staining positive for GLUT-1 (d) and GLUT-3 (e). 


\begin{tabular}{|c|c|c|c|}
\hline IHC staining of surgical specimen & $\begin{array}{l}\text { low } S U V_{\max } \\
(n=32)\end{array}$ & $\begin{array}{l}\text { high SUV } \max \\
(n=70)\end{array}$ & $p$-value \\
\hline $\begin{array}{l}\text { HIF-1a } \\
\text { (\% of tumours positive) }\end{array}$ & $37.9 \%$ & $63.1 \%$ & $p=0.024^{a}$ \\
\hline $\begin{array}{l}\text { CAIX } \\
\text { (\% of tumours positive) }\end{array}$ & $30.0 \%$ & $45.3 \%$ & $p=0.16^{a}$ \\
\hline $\begin{array}{l}\text { GLUT }-1 \\
\text { (\% of tumours positive) }\end{array}$ & $62.5 \%$ & $82.9 \%$ & $p=0.025^{a}$ \\
\hline $\begin{array}{l}\text { GLUT-3 } \\
\text { (\% of tumours positive) }\end{array}$ & $53.1 \%$ & $40.0 \%$ & $p=0.21^{a}$ \\
\hline $\begin{array}{l}\mathrm{Ki}-67 \text { (MIB) } \\
\text { (mean \% of cells positive) }\end{array}$ & $39.1 \%$ & $44.4 \%$ & $p=0.20^{b}$ \\
\hline
\end{tabular}

Table 3.3 Correlation between immunohistochemical staining patterns. Pearson correlation coefficients and p-value (between brackets) of immunohistochemical staining scores.

\begin{tabular}{|lcccc|}
\hline CAIX & HIF-1a & CAIX & GLUT-1 & GLUT-3 \\
& 0.36 & & & \\
GLUT-1 & $(<0.01)$ & & & \\
& 0.14 & 0.13 & & \\
GLUT-3 & $(0.19)$ & $(0.21)$ & -0.19 & \\
& 0.03 & 0.03 & $(0.85)$ & 0.17 \\
Ki-67 (MIB) & $(0.79)$ & $(0.80)$ & 0.20 & $(0.10)$ \\
& 0.33 & 0.36 & $(0.04)$ & \\
\hline
\end{tabular}

\section{Discussion}

Although the high FDG uptake in malignant tumours is due to an increased glucose metabolism, the exact mechanism by which FDG accumulates in malignant cells has not fully been unravelled ${ }^{17}$. Nevertheless, it was repeatedly shown that a high SUV $V_{\max }$ is related to an inferior overall survival $\left.\right|^{2-5,18}$.

This report studied the molecular mechanisms that might be involved in the prognostic

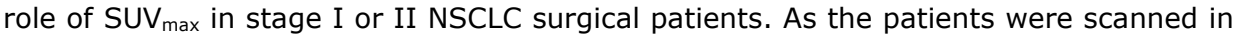
two departments, we used the best discriminative cut-off level for the SUV $\mathrm{Vax}_{\max }$ of each PET-camera to create a high and low $\mathrm{SUV}_{\max }$ group ${ }^{4,5}$. In this study we confirmed a worse survival for patients with a high FDG uptake compared with a low uptake (2-year survival of resp. $58.6 \%$ and $90.6 \%, p=0.001$ ). Furthermore, we investigated the biological characteristics of the tumours with a high and low FDG uptake. We observed an association between the amount of FDG uptake and the endogenous marker of hypoxia (HIF-1a) and the glucose transporter GLUT-1. Although it would be of interest to asses the amount of variance in FDG uptake explained by the expression of hypoxia related markers, this was not feasible since $S \mathrm{~V}_{\max }$ could not be used a continue variable due to the use of different scanners. Nevertheless, to the best of our knowledge we are the first to report an association between hypoxia related markers and SUV $_{\max }$ in lung cancer patients ${ }^{11,13,4}$. Indeed, Bos and colleagues investigated the correlation between different markers and the uptake of FDG in 55 breast tumours, but 
found no correlation between the HIF-1a and FDG uptake ${ }^{19}$. However, in their study a different cut-off point for HIF-1a expression (cut-off point of $1 \%$ ) was chosen.

In relation to the staining for HIF-1a and CAIX, we observed similar results as Giatromanolaki and colleagues showing $80 \%$ of CAIX positive cells also staining positive for HIF-1 $1 a^{6}$. Hypoxia up regulates different genes, like CAIX, GLUT-1 and GLUT-3, through the HIF-1a pathway ${ }^{20}$. However, different oxygen concentrations are required for induction of GLUT-1 and CAIX, which might explain the lack of association between SUV and CAIX, while finding a positive association between SUV and GLUT-1 ${ }^{21}$. For proliferative tumour activity, Veselle and colleagues showed in a study with 39 NSCLC patients a correlation between $\mathrm{Ki}-67$ expression and $\mathrm{SUV}_{\max }{ }^{22}$. In contrast, we did not observe a correlation between the proliferation marker Ki-67 and the SUV $\mathrm{max}_{\text {. Although }}$ the tumours with a high uptake showed a somewhat higher percentage of Ki-67 positivity, this difference did not reach significance $(p=0.20)$. In line with our results, Chung and colleagues found no correlation of PET findings and the proliferation index, measured by flowcytometry, in NSCLC ${ }^{23}$. Moreover, an animal model for prostate cancer, showed that a higher FDG uptake was indicative of tumour hypoxia but not for cellular proliferation ${ }^{14}$. Although the expression of GLUT is not necessarily directly related to transport activity, hypoxia can increase GLUT-1 levels and glucose uptake ${ }^{24}$. In several tumour sites, for instance cervix, oesophagus and breast, a relation between expression of glucose transporters and FDG uptake has been observed. In NSCLC contradictory findings have been observed. While the groups of Higashi and Mamede observed an association between the FDG uptake and GLUT-1, others did not find this correlation ${ }^{23,25-27}$. We could confirm a higher expression of GLUT-1 in the high SUV $\max$ tumours $(83 \%)$ compared with tumours with a low SUV $\max (63 \% ; p=0.025)$. The use of a different antibodies and/or different scoring methods may account for the difference in outcome of the studies.

In this study, a cut-off level of 8 and 11 respectively were found as best discriminative cut-off levels. Findings of different cut-off levels can be explained by the usage of different PET machines and techniques ${ }^{2-5}$. We stress that the above-mentioned cut-off levels are not generally applicable. To compare results between different scanners directly, calibrating is necessary. However, cut-off levels only represent a way to simplify a sliding scale in which a higher FDG uptake is associated with a worse survival. The goal of this study was to investigate the biological background of a high uptake of FDG, which is correlated with a worse outcome. Our results show that a high uptake of FDG is associated with a higher HIF-1a and GLUT-1 expression. This finding might have therapeutic implications. Both clinical and preclinical studies have described the role of HIF-1a in therapy resistance ${ }^{28}$. Potential hypoxia-targeted therapies, like blockade of HIF-1a, are under investigation ${ }^{20,28,29}$. In the future FDG-PET scans might be used for both selection of patients for different therapies and monitoring tumour response on these therapies.

In conclusion, the amount of uptake of FDG on the PET scan, as measured by the SUV $_{\max }$, is associated with the expression of HIF-1a and GLUT-1, both up regulated under hypoxic conditions. This study provides evidence that not only in vitro, but also in vivo hypoxia is associated with an increase in FDG uptake. 


\section{References}

1. Myrdal G, Lambe M, Gustafsson G, et al. Survival in primary lung cancer potentially cured by operation: influence of tumor stage and clinical characteristics. Ann Thorac Surg 2003;75:356363.

2. Borst GR, Belderbos JS, Boellaard R, et al. Standardised FDG uptake: a prognostic factor for inoperable non-small cell lung cancer. Eur J Cancer 2005;41:1533-1541.

3. Downey RJ, Akhurst T, Gonen M, et al. Preoperative F-18 fluorodeoxyglucose-positron emission tomography maximal standardized uptake value predicts survival after lung cancer resection. $J$ Clin Oncol 2004;22:3255-3260.

4. Eschmann SM, Friedel G, Paulsen F, et al. Is standardised (18)F-FDG uptake value an outcome predictor in patients with stage III non-small cell lung cancer? Eur $\mathrm{J}$ Nucl Med Mol Imaging 2005.

5. Vansteenkiste JF, Stroobants SG, Dupont PJ, et al. Prognostic importance of the standardized uptake value on (18)F-fluoro-2-deoxy-glucose-positron emission tomography scan in nonsmall-cell lung cancer: An analysis of 125 cases. Leuven Lung Cancer Group. J Clin Oncol 1999;17:3201-3206.

6. Giatromanolaki A, Koukourakis MI, Sivridis E, et al. Expression of hypoxia-inducible carbonic anhydrase- 9 relates to angiogenic pathways and independently to poor outcome in non-small cell lung cancer. Cancer Res 2001;61:7992-7998.

7. Hanahan D, Weinberg RA. The hallmarks of cancer. Cell 2000;100:57-70.

8. Swinson DE, Jones JL, Richardson D, et al. Carbonic anhydrase IX expression, a novel surrogate marker of tumor hypoxia, is associated with a poor prognosis in non-small-cell lung cancer. $J$ Clin Oncol 2003;21:473-482.

9. Vaupel P. The role of hypoxia-induced factors in tumor progression. Oncologist 2004;9 Suppl 5:10-17.

10. Zhang JZ, Behrooz A, Ismail-Beigi F. Regulation of glucose transport by hypoxia. Am J Kidney Dis $1999 ; 34: 189-202$.

11. Burgman $\mathrm{P}$, Odonoghue JA, Humm JL, et al. Hypoxia-Induced increase in FDG uptake in MCF7 cells. J Nucl Med 2001;42:170-175.

12. Thomas GV, Tran C, Mellinghoff IK, et al. Hypoxia-inducible factor determines sensitivity to inhibitors of mTOR in kidney cancer. Nat Med 2006;12:122-127.

13. Minn $\mathrm{H}$, Clavo AC, Wahl RL. Influence of hypoxia on tracer accumulation in squamous-cell carcinoma: in vitro evaluation for PET imaging. Nucl Med Biol 1996;23:941-946.

14. Pugachev A, Ruan S, Carlin S, et al. Dependence of FDG uptake on tumor microenvironment. Int J Radiat Oncol Biol Phys 2005;62:545-553.

15. Koukourakis MI, Bentzen SM, Giatromanolaki A, et al. Endogenous Markers of Two Separate Hypoxia Response Pathways (hypoxia inducible factor 2 alpha and carbonic anhydrase 9) Are Associated With Radiotherapy Failure in Head and Neck Cancer Patients Recruited in the CHART Randomized Trial. J Clin Oncol 2006.

16. Giatromanolaki A, Koukourakis MI, Sivridis E, et al. Relation of hypoxia inducible factor 1 alpha and 2 alpha in operable non-small cell lung cancer to angiogenic/molecular profile of tumours and survival. Br J Cancer 2001;85:881-890.

17. Thie JA. Understanding the standardized uptake value, its methods, and implications for usage. J Nucl Med 2004;45:1431-1434.

18. Gagel B, Reinartz P, Demirel C, et al. [18F] fluoromisonidazole and [18F] fluorodeoxyglucose positron emission tomography in response evaluation after chemo-/radiotherapy of non-smallcell lung cancer: a feasibility study. BMC Cancer 2006;6:51.

19. Bos R, van Der Hoeven JJ, van Der Wall E, et al. Biologic correlates of (18)fluorodeoxyglucose uptake in human breast cancer measured by positron emission tomography. J Clin Oncol 2002;20:379-387.

20. Harris AL. Hypoxia--a key regulatory factor in tumour growth. Nat Rev Cancer 2002;2:38-47.

21. Sorensen BS, Hao J, Overgaard J, et al. Influence of oxygen concentration and $\mathrm{pH}$ on expression of hypoxia induced genes. Radiother Oncol 2005;76:187-193. 
22. Vesselle H, Schmidt RA, Pugsley JM, et al. Lung cancer proliferation correlates with [F18]fluorodeoxyglucose uptake by positron emission tomography. Clin Cancer Res 2000;6:38373844.

23. Chung JK, Lee YJ, Kim SK, et al. Comparison of [18F]fluorodeoxyglucose uptake with glucose transporter-1 expression and proliferation rate in human glioma and non-small-cell lung cancer. Nucl Med Commun 2004;25:11-17.

24. Macheda ML, Rogers S, Best JD. Molecular and cellular regulation of glucose transporter (GLUT) proteins in cancer. J Cell Physiol 2005;202:654-662.

25. Higashi K, Ueda Y, Sakurai A, et al. Correlation of Glut-1 glucose transporter expression with [18F] FDG uptake in non-small cell lung cancer. Eur J Nucl Med 2000;27:1778-1785.

26. Mamede M, Higashi T, Kitaichi M, et al. [18F]FDG uptake and PCNA, Glut-1, and Hexokinase-II expressions in cancers and inflammatory lesions of the lung. Neoplasia 2005;7:369-379.

27. Marom EM, Aloia TA, Moore MB, et al. Correlation of FDG-PET imaging with Glut-1 and Glut-3 expression in early-stage non-small cell lung cancer. Lung Cancer 2001;33:99-107.

28. Moeller BJ, Dreher MR, Rabbani ZN, et al. Pleiotropic effects of HIF-1 blockade on tumor radiosensitivity. Cancer Cell 2005;8:99-110.

29. Semenza GL. Targeting HIF-1 for cancer therapy. Nat Rev Cancer 2003;3:721-732. 


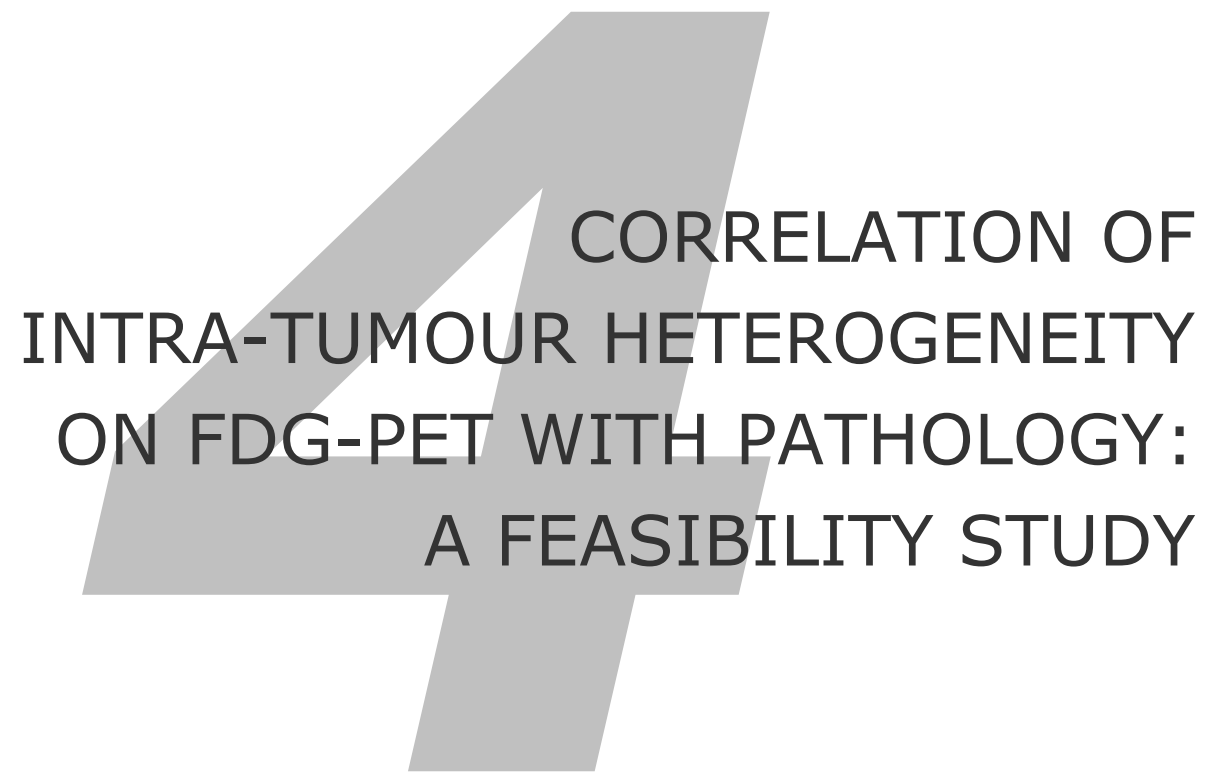

A. van Baardwijk, G. Bosmans, R.J. van Suylen, M. van Kroonenburgh, M. Hochstenbag, G. Geskes, P. Lambin and D. De Ruysscher. Correlation of intra-tumour heterogeneity on ${ }^{18}$ F-FDG PET with pathologic features in non-small cell lung cancer: a feasibility study.

Radiat Oncol 2008 (in press) 


\section{Abstract}

We evaluated the feasibility to correlate intra-tumour heterogeneity as visualized on ${ }^{18} \mathrm{~F}-\mathrm{FDG}$ PET with histology for non-small cell lung cancer. For this purpose we used an ex-vivo model. The procedure was feasible in all operated patients. We have shown that this method is suitable for correlating intra-tumour heterogeneity in tracer uptake with histology. 


\section{Introduction}

Positron emission tomography (PET) in oncology has evolved as a valuable tool for staging of disease, to predict prognosis and in the evaluation of therapy response in non-small cell lung cancer (NSCLC) ${ }^{1}$. Moreover, the use of PET scan in radiotherapy planning of NSCLC has gained great interest ${ }^{2,3}$. This success is mainly based on the application of the glucose analogue ${ }^{18} \mathrm{~F}$-2-fluoro-deoxy-D-glucose (FDG). Most tumours exhibit enhanced glucose uptake, which might be related to a higher consumption of FDG, an increased hexokinase activity or a decreased phosphatase activity within the tumour. As tumours are heterogeneous for several biological characteristics, it is the ultimate aim to visualize the phenotypic or micro-environmental tumour characteristics by imaging ${ }^{4}$. Variations in the FDG uptake within the tumour can be assumed to reflect heterogeneity of ongoing pathophysiological processes. Therefore, PET might potentially be used to map different areas within the tumour with specific characteristics, and would allow for specific targeting and hence improvement of radiation treatment outcome in NSCLC. Although the uptake of FDG on PET scan has been correlated with biological variables, like the expression of GLUT-1 and HIF-1 $1 a^{5-7}$, detailed knowledge about the underlying characteristics of intra-tumour heterogeneity in the uptake of FDG is not available $e^{8,9}$. Therefore, we tested an ex-vivo model to investigate heterogeneity in uptake of FDG within the tumour. The model was accepted to be feasible if it would result in good quality images showing intra-tumour heterogeneity as well as good quality tissue sections, which could be used for immunohistochemical staining. In this chapter, we describe the model used and our experience in the first patients.

\section{Materials and Methods}

\section{Model}

An ex-vivo model was tested to correlate intra-tumour heterogeneity in FDG uptake on PET with histology. FDG enters the cell by the same membrane transport mechanism as glucose and is phosphorylated by hexokinase. Since FDG-6-phosphate does not undergo further metabolism, FDG is trapped within the cells ${ }^{10}$. The PET-CT scan of a surgical specimen (ex-vivo) will thus provide us information about the situation before operation (in-vivo), at the time of injection of the tracer. Using an ex-vivo model no tumour movements are encountered during scanning and this furthermore provides the possibility of multiple sampling.

\section{Description of the procedure}

\section{Injection of FDG}

Patients fasted for at least 6 hours before the injection of the radioactive tracer ${ }^{18}$ FDG. Plasma glucose levels were checked and if fasting levels $(<7.0 \mathrm{mmol} / \mathrm{l})$ were measured, a small dose of FDG ( $1 / 10$ of the diagnostic used dose) was injected intravenously. The dose was calculated according to the weight of the patient: (weight*0.4+2) MBq. A low dose of FDG was chosen to limit radiation exposure to the patient, surgeon and pathologist. In case of an injected dose of $40 \mathrm{MBq}$ the effective dose ('dose to the body') for the patient was calculated to be about $0.76 \mathrm{mSv}$ 
according to ICRP $80^{11}$. This is a small dose compared to the dose given for a diagnostic PET scan (340 MBq; $6.5 \mathrm{mSv}$ ). An effective dose of $20 \mu \mathrm{Sv}$ for the surgeon and pathologist per patient operated was assumed to be acceptable. In case of 25 procedures a year, this would result in a maximal effective dose of $0.5 \mathrm{mSv}$ a year, which compares well with the dose limit for radiological workers of $20 \mathrm{mSv}$ per year. To compensate for the lower injected dose of FDG, a longer scanning time of the specimen was needed to achieve PET images of good quality.

\section{Surgery}

Surgery was performed about one hour after injection of FDG, in the standard way by a cardiothoracic surgeon. Once the tumour was resected the surgical specimen was transported directly to the pathology department and subsequential sectioned in parallel slices of $1 \mathrm{~cm}$ thickness, including the total tumour.

\section{PET-CT imaging}

The slices were put between Perspex plates in vertical direction to facilitate scanning and put on ice to guarantee the quality of the specimen for pathologic examination afterwards. The PET-CT scanner used was a Biograph ${ }^{\mathrm{TM}}$ (SOMATOM $^{\circledR}$ Sensation 16 with an ECAT ACCEL PET scanner, Siemens) with a transaxial field of view of $58.2 \mathrm{~cm}$, an axial field of view of $16.2 \mathrm{~cm}$ and a spatial resolution of 6-7 mm. First a spiral CT scan was performed, which was used for attenuation correction of PET emission images. A PET scan was performed in 1 bed position encompassing the total specimen with a scanning time of 30 minutes, to correct for the low dose of FDG injected. The complete PET data set was reconstructed iteratively with a reconstruction increment of $5 \mathrm{~mm}$ to provide isotropic voxels.

\section{Delineation of tumour heterogeneity}

The total tumour was selected as a 3-D Region of Interest (ROI) in e.soft (version 5.0, Siemens, Erlangen, Germany). The maximal uptake of FDG within the tumour was calculated and contours were created for regions with $80 \%, 50 \%$ and $20 \%$ of maximal uptake. For each scanned slice a 1:1 plot was created depicting the different contours.

\section{Biopsies}

The plots were printed on a transparent sheet and used to guide sampling out of the different regions. In each individual tumour, biopsies (small tissue blocks of about $0.25 \mathrm{~cm}^{3}$ ) were taken from the regions with the highest uptake $(>80 \%)$, the lowest uptake $(<20 \%)$ and a median uptake $(50 \%)$. The biopsies were paraffin-embedded. After sampling the surgical specimen was processed for regular pathologic examination. Biopsies were cut into tissue sections of $4 \mu \mathrm{m}$ thickness for haematoxylin and eosin (H\&E) staining and for further immunohistochemical staining. H\&E stained slides were microscopically evaluated to examine whether they contained vital tumour cells, fibrotic tissue, inflammation and/or necrotic tissue. An example of the total procedure is depicted in Figure 4.1. 

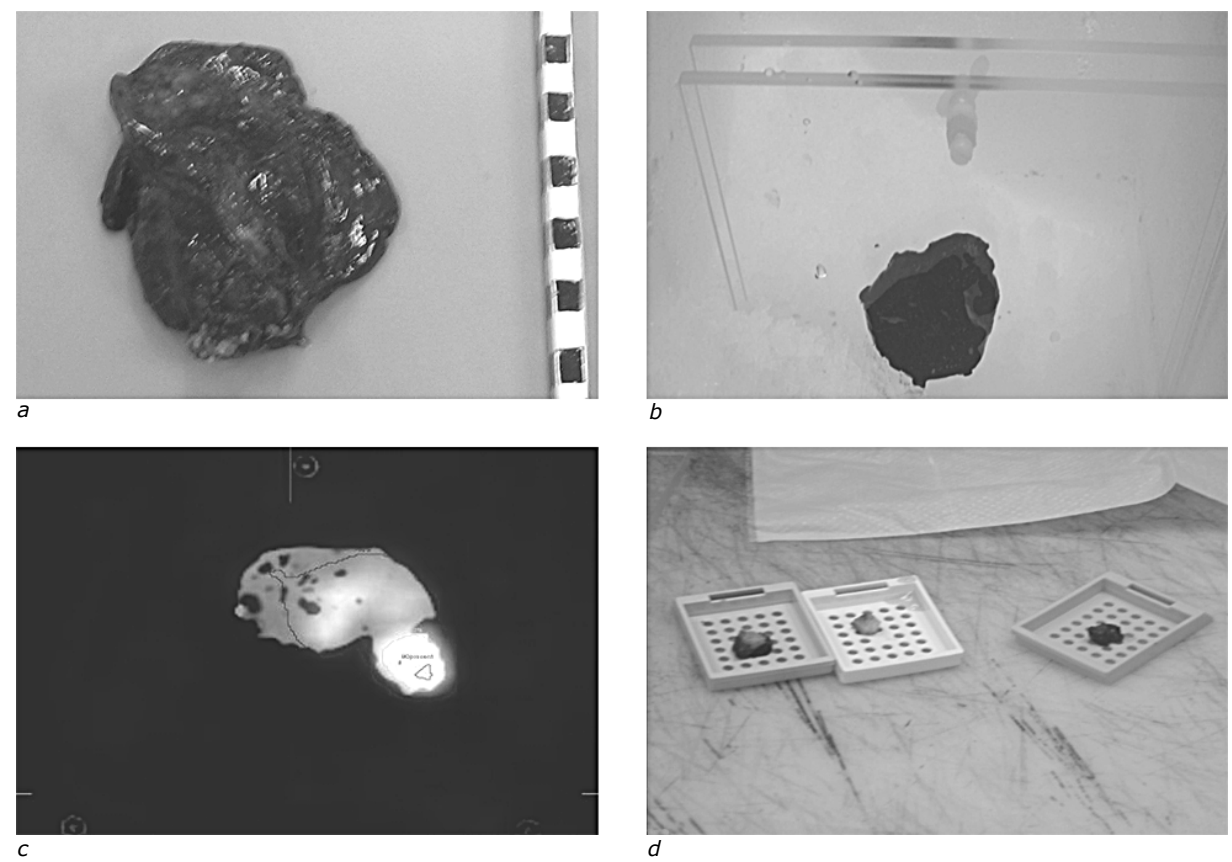

Figure 4.1 Visualization of the total procedure: total surgical specimen (a), specimen sectioned in parallel slices of $1 \mathrm{~cm}$ thickness ready to be scanned (b), delineation of tumour heterogeneity in e.soft with regions with $80 \%$ (pink line), 50\% (green line) and 20\% (red line) of maximal uptake of FDG (c) and biopsies from the different regions $(d)$.

\section{Patients}

Patients with operable NSCLC, who had not received previous chemotherapy or thoracic radiotherapy and did not suffer from diabetes mellitus, were studied. Five patients were assumed to be needed to test the feasibility. Tumours had to be histological proven NSCLC and all types except bronchiolo-alveolar cell carcinoma were accepted. To ensure intra-tumour heterogeneity tumours had to be PET positive on diagnostic imaging and at least $3 \mathrm{~cm}$ in diameter.

\section{Ethics}

All patients were treated at the University Hospital of Maastricht. This study has been reviewed and approved by the local Medical Ethical Committee, according to the Dutch law and regulations. All patients gave written informed consent before entering this study.

\section{Statistics}

SPSS software (SPSS for Windows, version 14.0, SPSS Inc., Chicago, IL) was used to perform statistical analysis. For the comparison of the contents of the different biopsies the Chi-square test for homogeneity was used. Two-tailed $p$-values are provided and p-values less than 0.05 were considered statistically significant. 


\section{Results}

\section{Patient and tumour characteristics}

The feasibility of the ex-vivo model was tested in 5 patients, 1 female and 4 male patients. The median age of the patients was 60 years (range $43-76$ years). The median diameter of the tumours was $3.0 \mathrm{~cm}$ (range $3.0-4.8 \mathrm{~cm}$ ). Four tumours consisted of a squamous cell carcinoma, where as one tumour was an adenocarcinoma.

\section{Feasibility}

In all cases it was feasible to slice and scan the tumour. A median dose of $36 \mathrm{Mbq}$ (range 34-46 Mbq) was injected. The interval between injection of FDG and completion of the resection of the tumour varied from 2 hours to 3.5 hours (median interval 120 minutes) and the median interval between resection and imaging was 60 minutes (range 30-90 minutes). A scanning time of 30 minutes was sufficient to visualize the tumour well. In all patients good quality images could be achieved showing intratumour heterogeneity of FDG uptake. The median maximal uptake of FDG within the tumour was $626.6 \mathrm{~Bq} / \mathrm{ml}$ (range 278.9-1037.7 Bq/ml). The surgical specimen was sectioned in a median number of 3 slices (range 3-4) to include the whole tumour for each patient. In total 23 biopsies were taken of the different regions with a median number of 5 biopsies (range 3-6) per tumour. In 3 out of 5 patients the highest uptake of FDG was observed in the peripheral zone of the tumour, while in 2 patients the highest uptake was centrally located. No clear relation between the localisation of the highest uptake and tumour diameter was observed. In total 7 samples were biopsied from low uptake regions, 9 from median uptake regions and 7 from high-utake regions.

\section{Radiation exposure}

The median effective dose for the 5 patients was $0.68 \mathrm{mSv}$ (range 0.64-0.95 mSv). For the surgeon the median effective dose was $9.4 \mu \mathrm{Sv}$ and for the pathologist $0.2 \mu \mathrm{Sv}$. This was below the dose of $20 \mu \mathrm{Sv}$, accepted as maximal dose for each procedure.

\section{Histological examination}

All biopsies showed a good quality of H\&E staining. Most slices consisted of a combination of tumour cells, fibrosis, inflammatory cells and/or necrosis. In total two samples out of the low uptake regions (29\%) showed no vital tumour cells. Although low uptake biopsies showed in a higher percentage mainly fibrosis $(43 \%)$ as the median $(11 \%)$ and high $(0 \%)$ uptake biopsies, the distribution patterns were not significantly different $(p=0.16$, Table 4.1).

\begin{tabular}{|c|c|c|c|}
\hline Characteristics & $\begin{array}{l}\text { low uptake of } \\
\text { FDG }\end{array}$ & $\begin{array}{l}\text { median uptake of } \\
\text { FDG }\end{array}$ & $\begin{array}{l}\text { low uptake of } \\
\text { FDG }\end{array}$ \\
\hline Mainly vital tumor (>75\%) & 2 & 1 & 2 \\
\hline Mainly fibrosis (>75\%) & 3* & 1 & 0 \\
\hline $\begin{array}{l}\text { Combination of vital tumour, fibrosis and/or inflammation } \\
\text { or necrosis }\end{array}$ & 2 & 7 & 5 \\
\hline
\end{tabular}




\section{Discussion}

Most studies correlated imaging with histology, investigated microscopic extension beyond CT-based borders ${ }^{12-14}$ or correlated the biological characteristics of a random tumour sample with the uptake of FDG of the total tumour ${ }^{5,7,15}$. Not much is known about the correlation between intra-tumour heterogeneity of FDG uptake and histology. The aim of this study was to test the feasibility to image relative intra-tumour heterogeneity in the uptake of FDG and the possibility to investigate the histological background of this phenomenon. Although both the removal of the tumour and the slicing might influence tumour architecture, FDG-6-phosphate does not undergo further metabolism and therefore FDG will be trapped within the cells ${ }^{10}$. The PET scan of the surgical specimen may thus represent the situation in-vivo. Sorensen et al. showed in a mice model, that in-vivo FDG PET was able to distinguish between viable tumour tissue and confluent areas of necrosis. This finding was verified by autoradiography and histological studies of the $\mathrm{C} 3 \mathrm{H}$ mammary carcinoma ${ }^{16}$. Zhao et al. performed a study in 7 rats, using an allogenic hepatoma cell model, to correlate intra-tumour heterogeneity in FDG distribution using autoradiography with the expression of several immunohistochemical markers ${ }^{9}$. A higher expression of FDG in the central region compared to peripheral zones of the tumour was observed. Applying our ex-vivo model, we observed in 3 out of 5 patients the highest uptake in the peripheral zone and in 2 patients this was centrally located. We did not observe a significant difference in distribution patterns concerning tumour, inflammation and fibrosis in these patients. Zhao et al. showed a correlation between the expression of the immunohistochemical markers Glut-1, Glut-3, Hexokinase II and intra-tumour accumulation of FDG. The hypoxia related marker Hypoxia Inducible Factor-1a (HIF-1a) was only observed in the central regions. This indicates that the heterogeneous distribution of FDG might be related to hypoxia, which leads to a higher expression of the glucosetransporters. This is in line with in- vitro and in-vivo studies that have demonstrated an increased uptake of FDG under hypoxic conditions $s^{7,17,18}$. Ongoing studies will investigate the relation between immunohistochemical staining patterns in relation to intra-tumour heterogeneity in FDG uptake on PET.

\section{Conclusions}

We demonstrated the feasibility of an ex-vivo method to correlate intra-tumour heterogeneity in vivo with histology in human NSCLC. This technique can also be applied to other tumour types and PET tracers, and can provide more insight information in the biological correlates of intra-tumour heterogeneity. Finally, this would allow for specific targeting and hence improvement of treatment outcome in NSCLC. 


\section{References}

1. Stroobants S, Verschakelen J, Vansteenkiste J. Value of FDG-PET in the management of nonsmall cell lung cancer. Eur J Radiol 2003;45(1):49-59.

2. van Baardwijk A, Baumert BG, Bosmans G, et al. The current status of FDG-PET in tumour volume definition in radiotherapy treatment planning. Cancer Treat Rev 2006;32(4):245-60.

3. Nestle U, Kremp S, Grosu AL. Practical integration of [18F]-FDG-PET and PET-CT in the planning of radiotherapy for non-small cell lung cancer (NSCLC): the technical basis, ICRU-target volumes, problems, perspectives. Radiother Oncol 2006;81(2):209-25.

4. Bentzen SM. Theragnostic imaging for radiation oncology: dose-painting by numbers. Lancet Oncol 2005;6(2):112-7.

5. Mamede M, Higashi T, Kitaichi M, et al. [18F]FDG uptake and PCNA, Glut-1, and Hexokinase-II expressions in cancers and inflammatory lesions of the lung. Neoplasia 2005;7(4):369-79.

6. Tateishi U, Yamaguchi U, Seki K, Terauchi T, Arai Y, Hasegawa T. Glut-1 expression and enhanced glucose metabolism are associated with tumour grade in bone and soft tissue sarcomas: a prospective evaluation by [18F]fluorodeoxyglucose positron emission tomography. Eur ] Nucl Med Mol Imaging 2006;33(6):683-91.

7. van Baardwijk A, Dooms C, van Suylen RJ, et al. The maximum uptake of (18)F-deoxyglucose on positron emission tomography scan correlates with survival, hypoxia inducible factor-1alpha and GLUT-1 in non-small cell lung cancer. Eur J Cancer 2007;43(9):1392-8.

8. Dearling JL, Flynn AA, Sutcliffe-Goulden J, et al. Analysis of the regional uptake of radiolabeled deoxyglucose analogs in human tumor xenografts. J Nucl Med 2004;45(1):101-7.

9. Zhao S, Kuge Y, Mochizuki T, et al. Biologic correlates of intratumoral heterogeneity in 18F-FDG distribution with regional expression of glucose transporters and hexokinase-II in experimental tumor. J Nucl Med 2005;46(4):675-82.

10. Pauwels EK, Ribeiro MJ, Stoot JH, McCready VR, Bourguignon M, Maziere B. FDG accumulation and tumor biology. Nucl Med Biol 1998;25(4):317-22.

11. International Commission on Radiological Protection. Radiation dose to Patients from Radiopharmaceuticals, Publication 80. Oxford: Pergamon Press; 1999.

12. Chan R, He Y, Haque A, Zwischenberger J. Computed tomographic-pathologic correlation of gross tumor volume and clinical target volume in non-small cell lung cancer: a pilot experience. Arch Pathol Lab Med 2001;125(11):1469-72.

13. Grills IS, Fitch DL, Goldstein NS, et al. Clinicopathologic Analysis of Microscopic Extension in Lung Adenocarcinoma: Defining Clinical Target Volume for Radiotherapy. Int J Radiat Oncol Biol Phys 2007.

14. Giraud P, Antoine M, Larrouy A, et al. Evaluation of microscopic tumor extension in non-smallcell lung cancer for three-dimensional conformal radiotherapy planning. Int J Radiat Oncol Biol Phys 2000;48(4):1015-24.

15. Tateishi U, Yamaguchi U, Seki K, Terauchi T, Arai Y, Hasegawa T. Glut-1 expression and enhanced glucose metabolism are associated with tumour grade in bone and soft tissue sarcomas: a prospective evaluation by [(18)F]fluorodeoxyglucose positron emission tomography. Eur J Nucl Med Mol Imaging 2006;33(6):683-91.

16. Sorensen M, Horsman MR, Cumming P, Munk OL, Keiding S. Effect of intratumoral heterogeneity in oxygenation status on FMISO PET, autoradiography, and electrode Po2 measurements in murine tumors. Int J Radiat Oncol Biol Phys 2005;62(3):854-61.

17. Pugachev A, Ruan S, Carlin S, et al. Dependence of FDG uptake on tumor microenvironment. Int J Radiat Oncol Biol Phys 2005;62(2):545-53.

18. Thomas GV, Tran C, Mellinghoff IK, et al. Hypoxia-inducible factor determines sensitivity to inhibitors of mTOR in kidney cancer. Nat Med 2006;12(1):122-7. 




\section{FDG-PET scan in

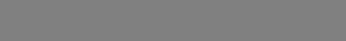

\section{radiotherapy of NSCLC}

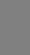

tang

The role of

FDG-PET scan in and (n)

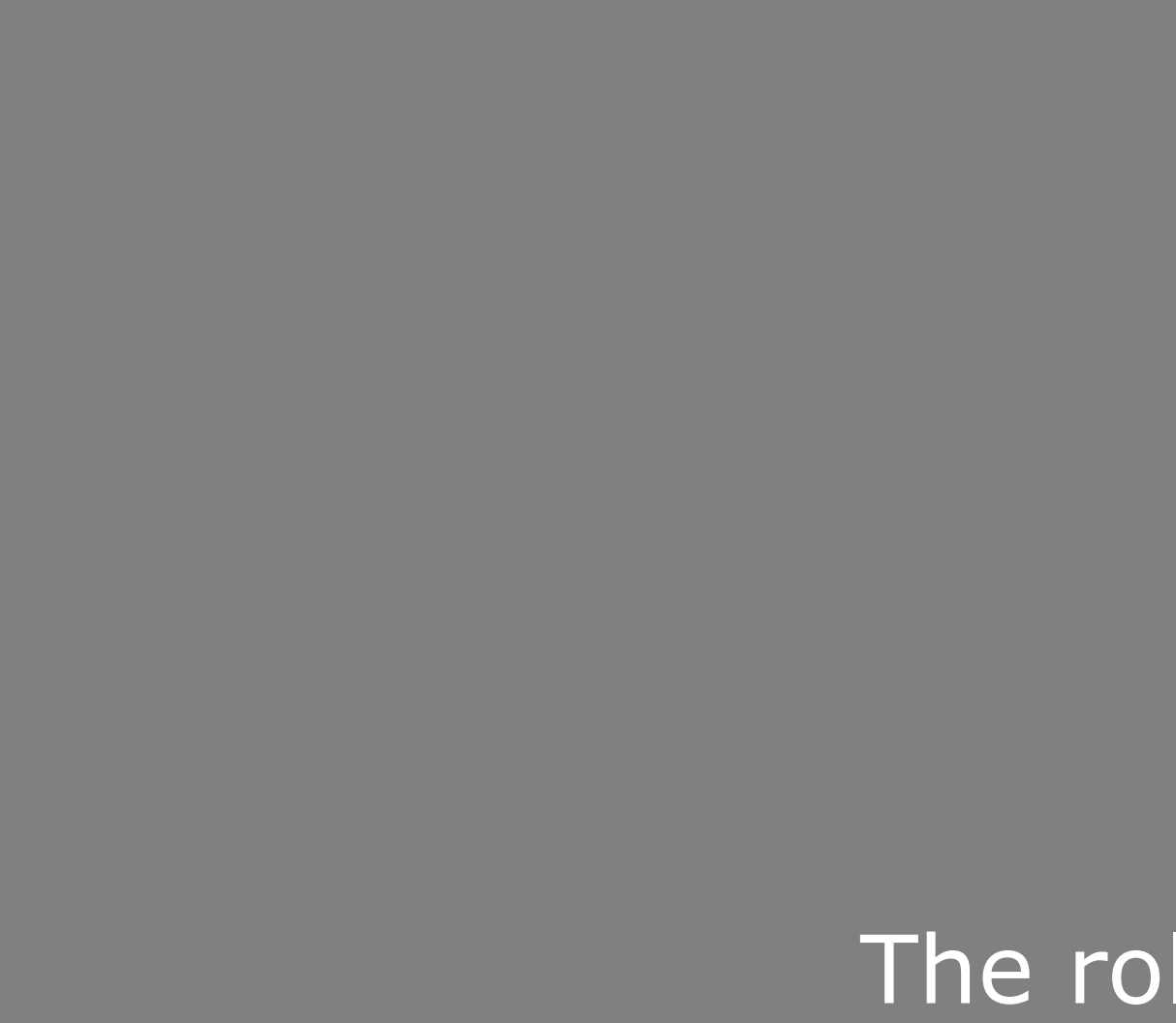

,





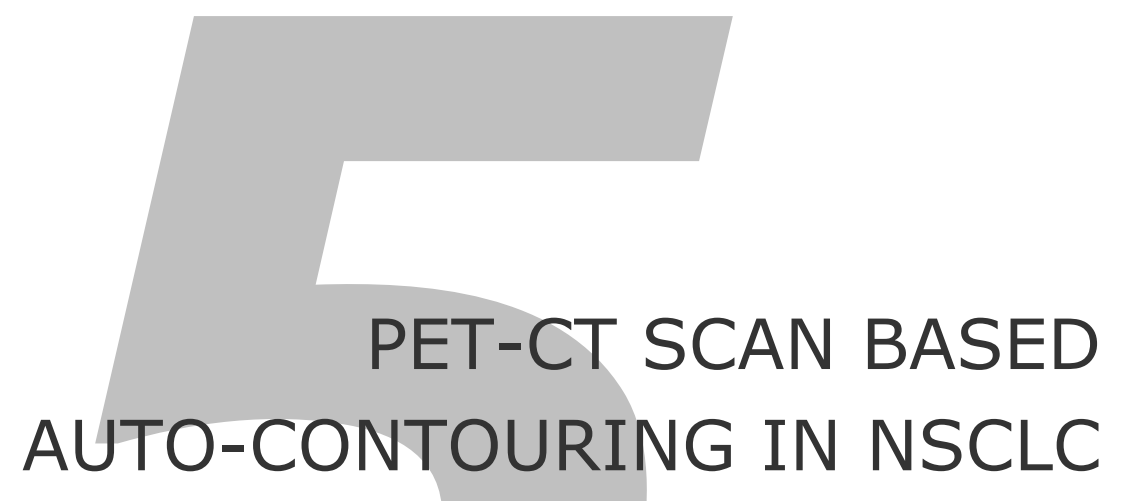

A. van Baardwijk, G. Bosmans, L. Boersma, J. Buijsen, S. Wanders, M. Hochstenbag, R.J. van Suylen, A. Dekker, C. Dehing-Oberije, R. Houben, S.M. Bentzen, M. van Kroonenburgh, P. Lambin and D. De Ruysscher. PET-CT scan based auto-contouring in non-small cell lung cancer correlates with pathology and reduces interobserver variability in the delineation of the primary tumor and involved nodal volumes.

Int J Radiat Oncol Biol Phys 2007;68(3):771-8 


\section{Abstract}

Purpose: To compare the source-to-background ratio (SBR) based PET-CT autodelineation with pathology in NSCLC and to investigate whether auto-delineation reduces the interobserver variability compared with manual PET-CT based gross tumor volume (GTV) delineation.

Material and Methods: SBR based auto-delineation was compared with macroscopic tumor dimensions to assess its validity in 23 tumors. Thereafter, GTVs were delineated manually on 33 PET-CT scans by 5 observers for the primary tumor (GTV-1 $1_{\operatorname{man}}$ ) and the involved lymph nodes (GTV- $2_{\text {man }}$ ). The delineation was repeated after 6 months with the auto-contour provided. This contour was edited by the observers (GTV auto). For comparison the concordance index (CI) was calculated, defined as the ratio of intersection and the union of 2 volumes $(A \cap B) /(A \cup B)$.

Results: The maximal tumor diameter of the SBR based auto-contour correlated strongly with the macroscopic diameter of primary tumors (correlation coefficient: 0.90 ) and was shown to be accurate for involved lymph nodes (sensitivity $67 \%$, specificity $95 \%$ ). The median auto-contour based target volumes were smaller than those defined by manual delineation for GTV-1 (31.8 cc and $34.6 \mathrm{cc}$, respectively, $\mathrm{p}=0.001)$ and GTV-2 (16.3 cc and $21.8 \mathrm{cc}$ respectively, $\mathrm{p}=0.02)$. The auto-contour based method showed higher CIs than the manual method for GTV-1 ( 0.74 and $0.70 \mathrm{cc}$ respectively, $p<0.001)$ and GTV-2 $(0.60$ and 0.51 cc respectively, $p=0.11)$.

Conclusions: SBR based auto-delineation showed a good correlation with pathology, decreased the delineated volumes of the GTVs and reduced the interobserver variability. Auto-contouring may further improve the quality of target delineation in NSCLC patients. 


\section{Introduction}

Thoracic radiotherapy plays an important role in the management of inoperable nonsmall cell lung cancer (NSCLC). The prognosis of patients with NSCLC remains poor due to high rates of local failure and distant metastases ${ }^{1-3}$. Although local tumor control can be improved by adding chemotherapy to radiotherapy and by increasing the total radiation dose, normal tissue toxicity is dose limiting ${ }^{4-9}$. A precise and consistent delineation of the target volumes is therefore a prerequisite for accurate radiation treatment planning. At present, target volume delineation is not only time consuming, but a large interobserver variability has been observed for many tumor sites ${ }^{10-19}$. Delineation accuracy can be improved using a standardized delineation protocol and by using Positron Emission Tomography (PET) information ${ }^{10,11,14,20-30}$. To date, at least 5 studies have investigated the role of integrating PET into target volume delineation in lung cancer and these have consistently shown a reduction in interobserver variation compared with CT-based delineation ${ }^{10,26,31-33}$. However, in all the currently used procedures, target volume delineation is carried out manually.

PET scan signals, based on Standardized Uptake Value (SUV) and Source-toBackground ratio (SBR) can be utilized to automatically delineate tumors. It is conceivable that these auto-delineation tools may improve the accuracy of target volume delineation. Different authors mentioned different fixed threshold values of maximal uptake, which vary from $36 \%$ to $50 \%$, as well as a variable threshold depending on the $\mathrm{SBR}^{31,34-37}$. The latter method has been compared with the 'gold standard' of pathology of surgical specimens of laryngeal carcinomas, where it has shown a complex, but accurate relation. In this tumor site the SBR based method underestimated, like other imaging modalities, contralateral mucosal extension, while overestimating infiltration of the cartilage and extralaryngeal structures ${ }^{38}$. To the best of our knowledge, no such study has been performed in lung cancer.

This study aimed to investigate the validity of SBR based auto-delineation, compared with the pathology in NSCLC, and subsequently to look at whether auto-contour based delineation would reduce interobserver variability in the delineation of the gross tumor volume (GTV) of the primary tumor and involved lymph nodes compared with conventional, manual PET-CT based delineation in patients with untreated NSCLC.

\section{Materials and Methods}

\section{PET-CT scan}

Included in this study were 33 consecutive patients with histological proven NSCLC (clinical stage Ib-IIIb), who had undergone a diagnostic whole body PET-CT scan (Biograph $^{\mathrm{TM}}$, SOMATOM ${ }^{\circledR}$ Sensation 16 with an ECAT ACCEL PET scanner, Siemens). Patients fasted at least 6 hours before the start of the examination. ${ }^{18} \mathrm{~F}$-fluoro-2-deoxyglucose (FDG) (MDS Nordion, Liège, Belgium) was injected intravenously, followed by physiologic saline $(10 \mathrm{ml})$. The injected total dose of FDG was dependent on the weight of the patient (weight* $4+20 \mathrm{MBq}$ ). After 45 minutes (time which is needed for uptake of FDG) free-breathing PET and CT images were acquired. First a topogram was made 
from the skull to the upper region of the legs. Secondly, a spiral CT-scan with intravenous contrast was performed. The PET images were acquired in 5 minute bed positions (in total 7 bed positions with overlap). The CT dataset was used for attenuation correction of PET images. The complete data set was then reconstructed iteratively with a reconstruction increment of $5 \mathrm{~mm}$ to provide isotropic voxels. The Standardized Uptake Value (SUV) was used to measure the amount of uptake of FDG in a selected Region of Interest (ROI). For the determination of the SUV of the primary tumor, a ROI was drawn on the 3D-images in e.soft (version 5.0.24.5, Siemens, Erlangen, Germany) around the region of the primary tumor. The SUV was calculated as activity concentration of FDG uptake divided by injected dose/body weight ${ }^{39}$. To minimize partial volume effects, the maximal SUV ( $S U V_{\max }$ ) within this ROI was calculated. PET images were interpreted and reported by a nuclear medicine physician. Three series of tomograms (transverse, coronal and sagital) were inspected simultaneously. Any focally enhanced ${ }^{18} \mathrm{~F}$-FDG uptake, that projected onto a pulmonary mass or a lymph node on CT were classified as positive ${ }^{40}$.

\section{SBR based auto-delineation}

The e.soft image processing software was used to delineate volumes exceeding a specified threshold automatically. To determine thresholds, phantom measurements were performed using different source-to-background ratios, as described by Daisne et al. ${ }^{5}$. A spherical phantom filled with 6 spheres with volumes ranging from 0.5 to $32 \mathrm{ml}$ was used. Each sphere was filled with ${ }^{18}$ F-FDG in different concentrations, together with different background concentrations to get different SBRs. The thresholds were determined as a percentage of the maximal uptake in the spheres, when the estimated volume as determined in e.soft was equal to the original volume of the sphere. According to these phantom measurements the threshold of FDG-PET activity varied from $34 \%$ to $47 \%$. In case of an SBR $>5$ an $34 \%$ threshold of maximal uptake could be used. For each individual patient this SBR was calculated, using the lung or the homolateral thoracic erector spinae muscle as relevant background depending on the localization of the primary tumor. The maximal SUV was measured within the primary tumor and the same threshold was used for both the primary tumor and the lymph nodes within the same patient. For all patients the maximal diameter of the SBR based auto-contour for the primary tumor was measured. The maximal diameter was measured on each slide in transversal, anterior-posterior and in cranio-caudal direction. For correlation with the pathological specimen the maximal diameter in any direction on PET scan was noted. With regard to the lymph nodal staging all lymph nodes showing a SBR based auto-contour were considered positive. The contour not necessarily needed to encompass the lymph node as a whole.

\section{Pathology}

Twenty-three of the 33 patients (70\%) underwent a surgical resection of their lung tumor. In these cases the maximal diameter of the primary tumor was measured by macroscopic examination. In all 33 patients, histological information concerning the lymph nodes was available. In 6 patients information was available by mediastinoscopy, in 24 patients by thoracotomy (in 2 patients after mediastinoscopy) and in 5 patients by trans-esophageal fine-needle aspiration. In total 69 lymph nodes (58 
mediastinal nodes and 11 hilar nodes) were examined, of which 9 mediastinal nodes showed metastases.

\section{Gross Tumor Volume delineation and interobserver variability}

Manual delineation based on fused PET-CT images was compared with the auto-contour based delineation. The PET-CT scans of all 33 patients were used for manual delineation of the primary tumor (GTV-1 $1_{\text {man }}$ ) and the involved lymph nodes (GTV-2 $2_{\text {man }}$ ), using a standard clinical delineation protocol (Figure 5.1a and Figure 5.1c). Both the PET-CT report and clinical information concerning these patients were provided. The primary tumor was delineated using the combined CT and PET information. In patients with a primary tumor growing into the mediastinum, only one combined GTV (GTV1+2) was delineated. GTV-2 only included lymph nodes that were PET positive and/or pathologically proven malignant. The protocol also included fixed window-level settings of both CT (lung W 1700; L -300, mediastinum W 600; L 40) and PET scan (W 30000; $L 15000$ ) to be used for delineation. For the final delineation of the lymph nodal areas the CT scan provided a better contrast with surrounding (fat) tissue and was mainly used for the determination of the border of the lymph node station.

Five observers independently performed the delineation: 3 radiation oncologists specialized in thoracic oncology and 2 experienced residents. All observers were blinded to the contours delineated by the others.

Target volume delineation based on PET-CT scan was repeated after 6 months, this time using an SBR based auto-contour provided to the observers, which they were asked to edit if they wished to do so both for the primary tumor (GTV-1 $1_{\text {auto }}$ ), the lymph nodes (GTV-2 $2_{\text {auto }}$ ) or the combined contour (GTV1+2 $2_{\text {auto; }}$ Figure 5.1b and Figure 5.1d).

Pairs of edited auto-contours and pairs of manually outlined volumes for the different observers were compared by the concordance index (CI), defined as the ratio of intersection and the union of the 2 volumes (Figure 5.2$)^{32,41}$.

$$
C I=\frac{(A \cap B)}{(A \cup B)}
$$

In which A stands for the GTV of the first observer and B for the GTV of the second observer. The CI was computed in 3-D for all tumors for all pairs of observers. The CI varies between 0 (no overlapping volumes between 2 observers) and 1 (complete agreement on delineated volume).

\section{Statistics}

SPSS software (SPSS for Windows, version 14.0, SPSS Inc., Chicago, IL) was used to perform statistical analysis. Pearson's correlation coefficient was used to compare pathology and imaging estimates of maximal tumor diameter.

To compare sensitivity and specificity with regard to lymph nodal staging of the Nuclear Medicine report and the SBR based auto-contouring method, the areas under the curves of the Receiver Operating Characteristic (ROC) curves were compared using 
the method described by Hanley and $\mathrm{Mc} \mathrm{Neil}^{42}$.Concerning the delineations, for each case, each observer and each method used, the different volumes were measured. Results are summarized by the median and range. To assess interobserver variation statistically, the coefficient of variation (CV) of the GTVs for each individual patient was measured. The CV is defined as the standard deviation (SD) divided by the mean GTV of the 5 observers. Moreover, the CIs of both methods were compared using the Wilcoxon signed ranks test, since the data did not follow a normal distribution. Twotailed $p$-values are provided and $p$-values less than 0.05 were considered statistically significant.
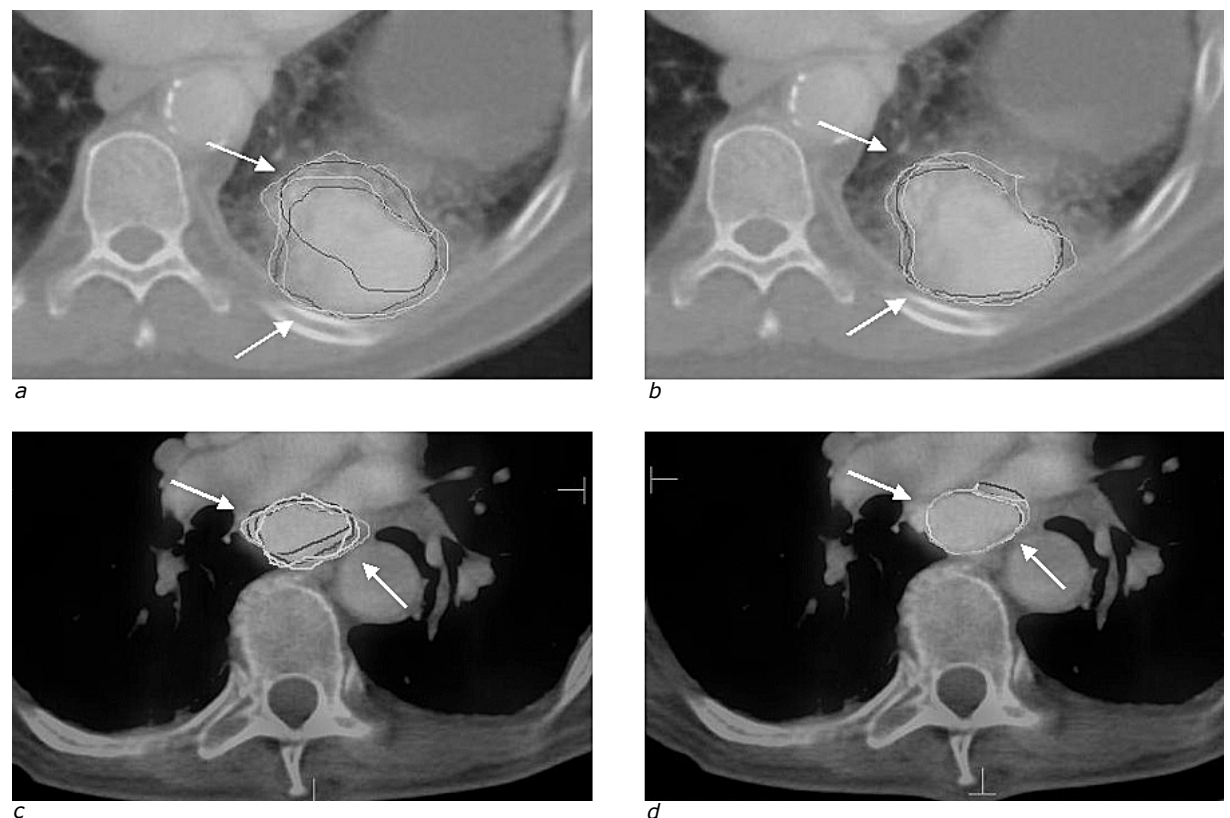

Figure 5.1 Example of manual (a) and auto-contour based (b) delineation of a primary tumor (GTV-1) and of manual (c) and auto-contour based (d) delineation of GTV-2 (lymph nodal area) delineated by the five observers. Arrows indicate changes in interobserver variation in delineation between the two methods.

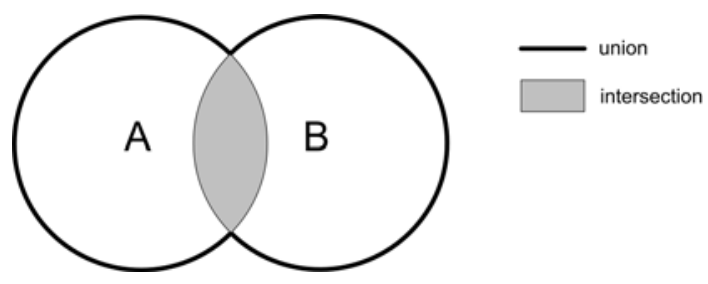

Figure 5.2 Concordance index: intersection of volume $A$ and $B$ divided by the union of volume $A$ and $B$. 


\section{Results}

\section{SBR based auto-contour compared with pathology}

In order to check validity the PET automatic SBR based delineation of the primary tumor was compared with macroscopic examination of the surgical specimen $(n=23)$. The maximal diameter of the tumor on macroscopy was compared with the maximal diameter of the PET auto-contour as estimated by e.soft (SBR based). A strong correlation was found between these two parameters (Pearson correlation coefficient 0.90 ; Figure 5.3).

In general the maximal diameter of the auto-contour slightly underestimated the maximal diameter found at macroscopic examination. The accuracy of the threshold based auto-contouring for hilar and mediastinal lymph node delineation was compared with the report of the PET scan (based on visual inspection, department of Nuclear Medicine). Compared with the gold standard of pathology, the auto-contouring method showed a sensitivity of $66.7 \%$ (95\% confidence interval: $35.1 \%-88.3 \%$ ) and a specificity of $95.0 \%$ ( $95 \%$ confidence interval: $85.8 \%-98.8 \%$ ), whereas the report of the department of Nuclear Medicine showed a sensitivity of $55.6 \%$ (95\% confidence interval: $26.6 \%-81.2 \%$ ) and a specificity of $88.3 \%$ ( $95 \%$ confidence interval: $77.5 \%-94.5 \%$ ) (AUC of ROC curve auto-contouring 0.81 , AUC of ROC curve Nuclear Medicine $0.72, p=0.28)$.

\section{SBR based delineation compared with manual delineation}

For 30 patients a GTV-1 was delineated, whereas for 3 patients with a tumor growing into the mediastinum a GTV $-1+2$ was delineated. In 10 patients additional lymph nodes, GTV-2, were contoured. In all patients the GTVs were delineated both manually and by editing the SBR based auto-contour. The results concerning volume are depicted in Table 5.1 .

The edited auto-contour of the primary tumor, GTV-1 $1_{\text {auto }}$ (median $31.8 \mathrm{cc}$ ) was smaller than the manually contoured GTV-1 (median $34.6 \mathrm{cc}, \mathrm{z}=-3.36, \mathrm{p}=0.001$ ). Similar results were found for the small group of tumors growing into the mediastinum (median GTV-1 $+2_{\text {auto }} 45.2 \mathrm{cc}$ and GTV1 $+2_{\operatorname{man}} 66.4 \mathrm{cc}$ ), although this difference was not significant $(z=-1.60, p=0.11)$. The median volume of delineated lymph nodes, GTV-2, was also significantly smaller using the auto-contour based method than the manual method $(z=-2.40, p=0.017)$. The median volume of GTV-2 auto was $16.3 \mathrm{cc}$ compared with GTV- $2_{\text {man }} 21.8 \mathrm{cc}$. For the individual observers, the changes in volumes for GTV-1, GTV-1+2 and GTV-2 showed similar results, although not all individual changes did reach significant levels (Table 5.1). To investigate whether the inter-observer agreement of the delineated volume would change using the auto-contouring method, we compared the coefficient of variation (CV) of the 2 methods for the different delineated volumes. The CV of the auto-contour based method was significantly lower than of the manual method for the primary tumors $(z=-3.76, p<0.001)$. The median CV was 0.15 for GTV-1 $1_{\text {auto }}$ and 0.22 for GTV-1 $1_{\text {man }}$. The changes in CV for each individual delineated GTV-1 is depicted in Figure 5.4. No significant change in CV was observed for GTV $-1+2$ $(z=-0.0, p=1.0)$ and GTV-2 $(z=-2.55, p=0.80)$ between the 2 methods. 


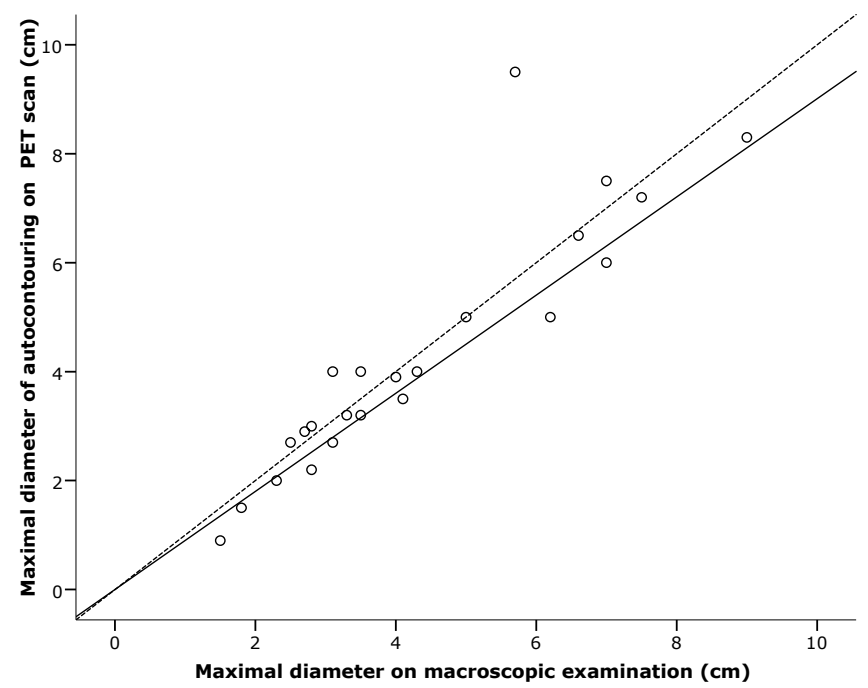

Figure 5.3 Maximal diameter of primary lung tumor assessed by PET scan (SBR based automatic delineation) compared with maximal diameter of tumor on macroscopic examination of the surgical specimen $(n=23)$. Pearson correlation coefficient $=0.90, r^{2}=0.82$. The solid line (_) represents the linear fit and the dotted line (---) the reference line.

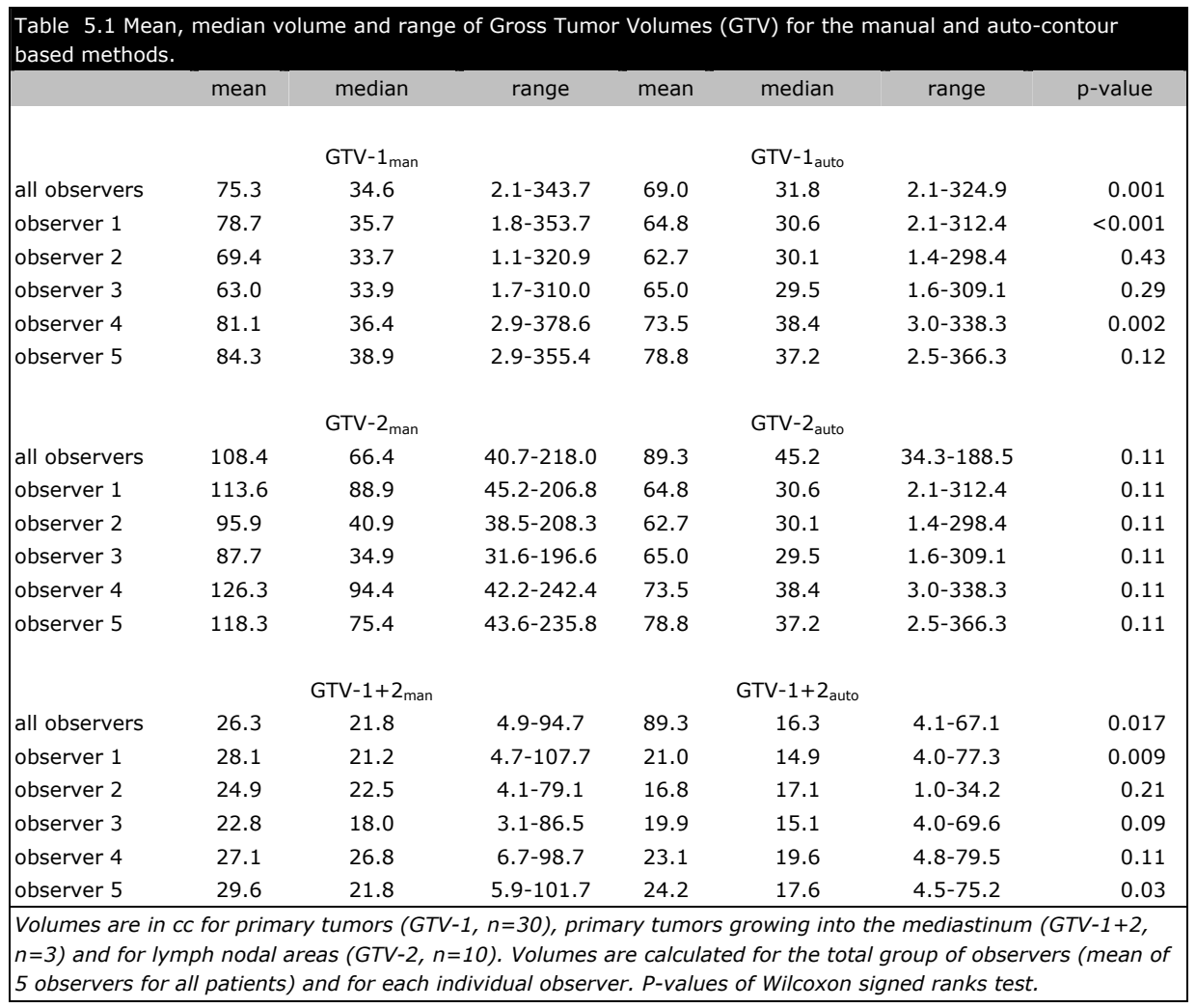


To investigate whether the inter-observer agreement of the delineated volume would change using the auto-contouring method, we compared the coefficient of variation (CV) of the 2 methods for the different delineated volumes. The CV of the auto-contour based method was significantly lower than of the manual method for the primary tumors $(z=-3.76, p<0.001)$. The median CV was 0.15 for $G T V-1_{\text {auto }}$ and 0.22 for GTV-1 $1_{\text {man. }}$. The changes in CV for each individual delineated GTV-1 is depicted in Figure 5.4. No significant change in CV was observed for GTV-1+2 $(z=0.0, p=1.0)$ and GTV-2 $(z=-2.55, p=0.80)$ between the 2 methods.

Evaluation of the interobserver variation using the concordance indices (CIs) showed similar results. The auto-contour based method showed higher CIs than the manual based method. For GTV-1 the auto-contour based method had a median CI of 0.74 , compared with a CI of 0.70 for the manual method $(z=-4.16, p<0.001)$. Although the CI for GTV-2 also showed an increase, this difference was not significant. The CI for GTV-2 showed a median of 0.60 for the auto-contouring method compared with 0.51 for the manual method $(z=-1.58, p=0.11)$. No difference was observed between the CIs of GTV-1+2 ( $z=-1.07, p=0.29$; Table 5.2).

\section{Discussion}

The delineation of target volumes, as well as organs at risk is a very critical step in high-precision radiation treatment planning ${ }^{43}$. Both good image quality as well as a good delineation protocol is crucial for target volume delineation. Different methods of PET(-CT) based contouring have been described in literature ${ }^{10,21,34-37,44}$. Visual interpretation and threshold based methods, using a fixed threshold, an SBR based or gradient based method, have been used to delineate tumors. Although the SBR based methods show a good agreement with pathologic-anatomic structures, these methods should be validated individually for each PET scanner used ${ }^{35}$. Nestle et al. compared 4 different PET-based delineation methods (visually, threshold of $40 \%$ of maximal SUV, SUV of 2.5 and a tumor-to-background algorithm) with CT-based volumes ${ }^{37}$. In this study the threshold of $40 \%$ was the only volume, which did not correlate with CT-based volume. However, no correlation with the macroscopic tumor diameter was attempted to validate the different methods. Actually, only few studies have compared the accuracy of imaging to pathology. For head-and-neck cancer Daisne et al. showed that automatic delineation on PET using the SBR-method was accurate in delineating laryngeal tumors ${ }^{38}$. Although the GTVs of the surgical specimen were on average smaller than the GTVs obtained by PET, CT and MRI, there was an overestimation of extralaryngeal extension and an underestimation of superficial spread of the tumor using the different imaging modalities. Comparing the 3 modalities, FDG PET showed the highest specificity. Although it is important to validate auto-delineation software in lung cancer as well, to date, no such a study has been performed in NSCLC. In the present study, we showed that the SBR based auto-contouring method was accurate in localizing the involved nodal areas. The specificity of $95 \%$ is comparable with published data on PET scan. The sensitivity of $67 \%$, found analyzing the small number (9/69) of positive lymph nodes in our study, is also in line with values described in literature, which range from $66 \%$ to $83 \%{ }^{45-47}$. 


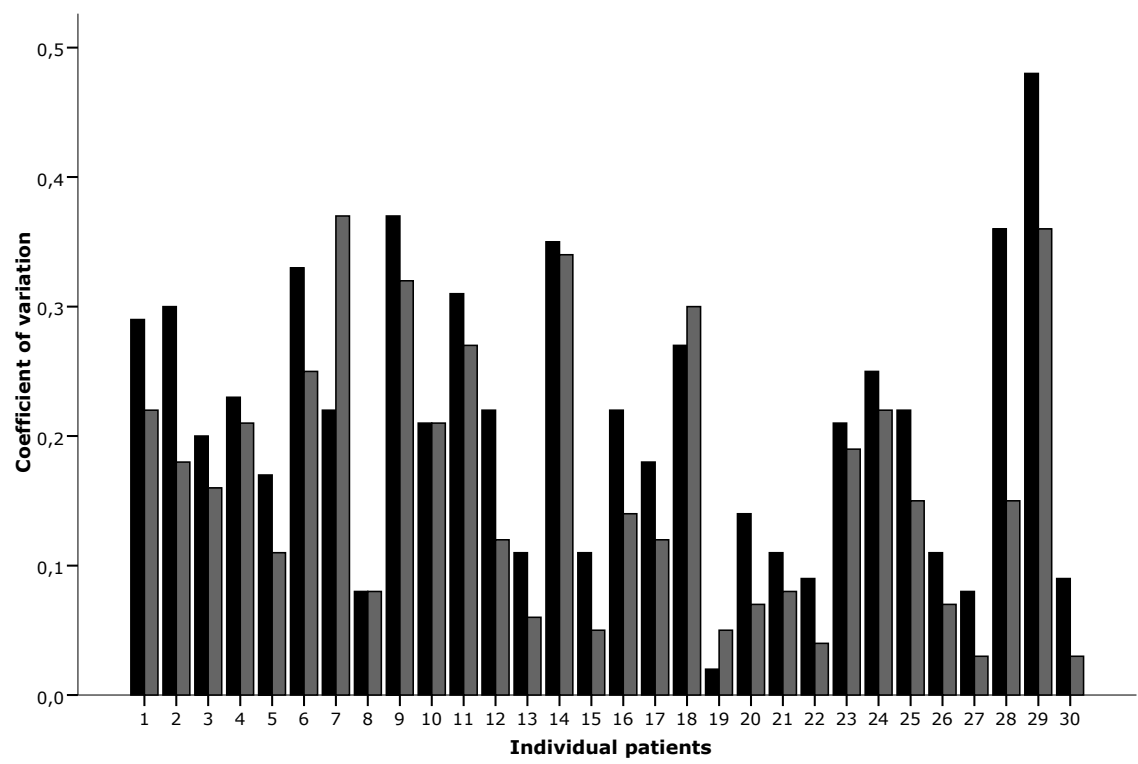

Figure 5.4 Coefficient of variation (CV) of GTV-1 for each individual patient for both the manual method (black bars) and the auto-contouring based method (grey bars).

Table 5.2 Mean, median volume and range of coefficient of variation (CV) and concordance index (CI) for the manual and auto-contour based methods.

\begin{tabular}{|c|c|c|c|c|c|c|c|}
\hline & mean & median & range & mean & median & range & $\mathrm{p}$-value \\
\hline & \multicolumn{3}{|c|}{ CVmanual } & \multicolumn{3}{|c|}{ CVauto-contour } & \\
\hline GTV-1 & 0.21 & 0.22 & $0.02-0.48$ & 0.17 & 0.15 & $0.03-0.37$ & $<0.001$ \\
\hline GTV $-1+2$ & 0.23 & 0.24 & $0.12-0.30$ & 0.22 & 0.19 & $0.05-0.50$ & 0.8 \\
\hline \multirow[t]{2}{*}{ GTV-2 } & 0.21 & 0.13 & $0.09-0.42$ & 0.21 & 0.12 & $0.06-0.47$ & 1.0 \\
\hline & & \multicolumn{3}{|c|}{ CImanual } & \multicolumn{3}{|c|}{ CIauto-contour } \\
\hline GTV-1 & 0.70 & 0.70 & $0.51-0.84$ & 0.74 & 0.74 & $0.57-0.88$ & $<0.001$ \\
\hline GTV-1+2 & 0.63 & 0.69 & $0.42-0.78$ & 0.67 & 0.69 & $0.49-0.84$ & 0.29 \\
\hline GTV-2 & 0.51 & 0.51 & $0.33-0.70$ & 0.59 & 0.60 & $0.43-0.85$ & 0.11 \\
\hline
\end{tabular}

It could be expected that a subpopulation of tumors, e.g. larger tumors, show a higher sensitivity of lymph nodal auto-contouring. However, due to the limited number of positive nodes this could not be tested in this study. We also observed a good correlation (correlation coefficient 0.90 ) between the maximal diameter on PET-scan using the SBR based auto-contouring method and the macroscopic tumor diameter on the surgical specimen for 23 operable lung cancers, although this is admittedly a fairly crude measure. A prospective study to further investigate the accuracy of PET-CT in the delineation of lung tumors, including shape and location of the tumor, compared with pathologic delineation is ongoing ${ }^{48}$. In the current study free-breathing PET-CT images were used for the delineation, showing smearing of the PET signal due to tumor motion. In the prospective study respiratory correlated imaging will be performed, 
which might further improve depiction of the real tumor volume and therefore can lead to a change in cut-off levels and/or segmentation methods used for auto-contouring.

Another important issue in target volume delineation is the interobserver variability. Therefore several authors investigated the influence of integrating PET into target volume delineation in lung cancer. Caldwell et al. found a reduction in the ratio of largest to smallest GTV using PET-CT co-registered data compared with CT alone in a group of 30 patients, delineation being performed by 3 observers ${ }^{31}$. A reduction in interobserver variation using matched PET-CT compared with CT was noted by Steenbakkers et al. and by Ashmalla and co-authors ${ }^{10,33}$. Fox et al. looked at the influence of registration of PET-CT images on the concordance. They showed an improved concordance using registered PET-CT images compared with side-by-side images (CI of 0.70 and 0.61 respectively) ${ }^{32}$. In our study, we found a CI of 0.70 for the primary tumor using manual delineation on PET-CT fused images and a further improvement of conformity ( $\mathrm{CI}$ 0.74) using the SBR based auto-contouring method $(p<0.001)$. The fact that the CIs were significantly lower than 1.0 , the value reached for perfect concordance reflects that individual observers were allowed to edit the autocontour based delineation to their wished contour. The edited contour will clearly be influenced by the delineation the way the observers are used to delineate, as has been performed in the manual method. However, we did observe an increase in CIs and also a significant decrease in CV among observers of the GTV-1 using the auto-contouring based method compared with the manual method $(p<0.001)$. This shows that there was less variation in the volume of GTV-1 delineated using this auto-contour based method compared with the standard manual way. Although our study set also included surgical patients, the mean volumes for the primary tumor in this series is comparable with other studies. The mean volume for GTV- $1_{\operatorname{man}}$ was $75.3 \mathrm{cc}$ (range 2.1-343.7 cc) in our study, while tumors in the Steenbakkers' study had a mean volume of 69 cc (range 4-307 cc) and in Caldwell's study of $90.5 \mathrm{cc}$ (range 12-360 cc) ${ }^{29,31}$. We therefore believe our data are representative for NSCLC patients, including patients referred for radiotherapy.

In our study we found a non-significant improvement in agreement between the observers in nodal volume delineation ( $\mathrm{CI}_{\operatorname{man}} 0.51$ and $\mathrm{CI}_{\text {auto }} 0.60, \mathrm{p}=0.11$ ). For the lymph nodal areas we observed somewhat lower CIs for the lymph nodal areas than for the primary tumor. However, in the literature, no separation between nodal volumes and volumes of the primary tumors were made, thus we cannot compare the present data with those of others.

The ultimate goal is a precise delineation of the tumor and a higher agreement between observers together with an accurate auto-contouring method will ultimately lead to better target volume delineation. One of the advantages of the auto-contour method is that it directly gives a possible delineation solution, which is visualized on screen for the radiation oncologist, who then only has to edit the contour. Another advantage of the auto-contour based delineation might be a reduction of the time needed to delineate. Steenbakkers et al. reported that including a PET scan in target volume delineation reduced the mean delineation time from 16 minutes to 12 minutes $(p<0.001)^{33}$. Although delineation time needed was not recorded in our study, there 
was a general feeling among the observers that editing the automatic contour was less time consuming than manual delineation based on PET-CT starting from scratch.

In conclusion, the use of a source-to-background ratio based automatic delineation showed a good correlation with macroscopic and histological examination in NSCLC, both for the maximal diameter of the primary tumor as well as for the involved lymph nodes. The SBR based automated contour reduced the interobserver variability, since lower CVs and higher CIs were found using the auto-contour based delineation. This approach may thus improve target volume delineation in daily practice radiation oncology. 


\section{References}

1. Ataman OU, Bentzen SM, Saunders MI, et al. Failure-specific prognostic factors after continuous hyperfractionated accelerated radiotherapy (CHART) or conventional radiotherapy in locally advanced non-small-cell lung cancer: a competing risks analysis. Br J Cancer 2001;85:11131118.

2. Novello S, le Chevalier T. Is there a standard strategy in the management of locally advanced non-small cell lung cancer? Lung Cancer 2001;34 Suppl 4:S9-14.

3. Sause W, Kolesar P, Taylor SI, et al. Final results of phase III trial in regionally advanced unresectable non-small cell lung cancer: Radiation Therapy Oncology Group, Eastern Cooperative Oncology Group, and Southwest Oncology Group. Chest 2000;117:358-364.

4. Cox JD, Azarnia N, Byhardt RW, et al. A randomized phase I/II trial of hyperfractionated radiation therapy with total doses of $60.0 \mathrm{~Gy}$ to $79.2 \mathrm{~Gy}$ : possible survival benefit with greater than or equal to $69.6 \mathrm{~Gy}$ in favorable patients with Radiation Therapy Oncology Group stage III non-small-cell lung carcinoma: report of Radiation Therapy Oncology Group 83-11. J Clin Oncol 1990;8:1543-1555.

5. Furuse K, Fukuoka M, Kawahara M, et al. Phase III study of concurrent versus sequential thoracic radiotherapy in combination with mitomycin, vindesine, and cisplatin in unresectable stage III non-small-cell lung cancer. J Clin Oncol 1999;17:2692-2699.

6. Hazuka MB, Turrisi AT, 3rd, Lutz ST, et al. Results of high-dose thoracic irradiation incorporating beam's eye view display in non-small cell lung cancer: a retrospective multivariate analysis. Int J Radiat Oncol Biol Phys 1993;27:273-284.

7. Kong FM, Ten Haken RK, Schipper MJ, et al. High-dose radiation improved local tumor control and overall survival in patients with inoperable/unresectable non-small-cell lung cancer: Longterm results of a radiation dose escalation study. Int J Radiat Oncol Biol Phys 2005;63:324333.

8. Martel MK, Ten Haken RK, Hazuka MB, et al. Estimation of tumor control probability model parameters from 3-D dose distributions of non-small cell lung cancer patients. Lung Cancer 1999;24:31-37.

9. Onishi H, Kuriyama K, Yamaguchi $M$, et al. Concurrent two-dimensional radiotherapy and weekly docetaxel in the treatment of stage III non-small cell lung cancer: a good local response but no good survival due to radiation pneumonitis. Lung Cancer 2003;40:79-84.

10. Ashamalla $\mathrm{H}$, Rafla $\mathrm{S}$, Parikh $\mathrm{K}$, et al. The contribution of integrated PET/CT to the evolving definition of treatment volumes in radiation treatment planning in lung cancer. Int J Radiat Oncol Biol Phys 2005;63:1016-1023.

11. Bowden P, Fisher R, Mac Manus M, et al. Measurement of lung tumor volumes using threedimensional computer planning software. Int J Radiat Oncol Biol Phys 2002;53:566-573.

12. Court LE, Dong L, Taylor N, et al. Evaluation of a contour-alignment technique for CT-guided prostate radiotherapy: an intra- and interobserver study. Int $\mathrm{J}$ Radiat Oncol Biol Phys 2004;59:412-418.

13. Geets X, Daisne JF, Arcangeli S, et al. Inter-observer variability in the delineation of pharyngolaryngeal tumor, parotid glands and cervical spinal cord: comparison between CT-scan and MRI. Radiother Oncol 2005; 77:25-31.

14. Hurkmans CW, Borger $\mathrm{JH}$, Pieters BR, et al. Variability in target volume delineation on CT scans of the breast. Int J Radiat Oncol Biol Phys 2001;50:1366-1372.

15. Riegel AC BA, Destian S, Ng T, Tena LB, Micnick RJ, Wong PS. Variability of gross tumor volume delineation in head-and-neck cancer using CT and PET/CT fusion. Int $J$ Radiat Oncol Biol Phys 2006;Apr 18; [Epub ahead of print].

16. Saarnak $A E$, Boersma $M$, van Bunningen $B N$, et al. Inter-observer variation in delineation of bladder and rectum contours for brachytherapy of cervical cancer. Radiother Oncol 2000;56:3742.

17. Tai P, Van Dyk J, Yu E, et al. Variability of target volume delineation in cervical esophageal cancer. Int J Radiat Oncol Biol Phys 1998;42:277-288.

18. Van de Steene J, Linthout N, de Mey J, et al. Definition of gross tumor volume in lung cancer: inter-observer variability. Radiother Oncol 2002;62:37-49. 
19. Yamamoto M, Nagata $Y$, Okajima K, et al. Differences in target outline delineation from CT scans of brain tumours using different methods and different observers. Radiother Oncol 1999;50:151-156.

20. Kiffer JD, Berlangieri SU, Scott AM, et al. The contribution of 18F-fluoro-2-deoxy-glucose positron emission tomographic imaging to radiotherapy planning in lung cancer. Lung Cancer 1998;19:167-177.

21. Mah K, Caldwell CB, Ung YC, et al. The impact of (18)FDG-PET on target and critical organs in CT-based treatment planning of patients with poorly defined non-small-cell lung carcinoma: a prospective study. Int J Radiat Oncol Biol Phys 2002;52:339-350.

22. Steenbakkers RJ, Duppen JC, Fitton I, et al. Observer variation in target volume delineation of lung cancer related to radiation oncologist-computer interaction: a 'Big Brother' evaluation. Radiother Oncol 2005;77:182-190.

23. van Baardwijk A, Baumert BG, Bosmans G, et al. The current status of FDG-PET in tumour volume definition in radiotherapy treatment planning. Cancer Treat Rev 2006;32:245-260.

24. Bradley J, Thorstad WL, Mutic S, et al. Impact of FDG-PET on radiation therapy volume delineation in non-small-cell lung cancer. Int J Radiat Oncol Biol Phys 2004;59:78-86.

25. Bujenovic S. The role of positron emission tomography in radiation treatment planning. Semin Nucl Med 2004;34:293-299.

26. Ciernik IF, Dizendorf E, Baumert BG, et al. Radiation treatment planning with an integrated positron emission and computer tomography (PET/CT): a feasibility study. Int $J$ Radiat Oncol Biol Phys 2003; 57:853-863.

27. De Ruysscher D, Wanders S, Minken A, et al. Effects of radiotherapy planning with a dedicated combined PET-CT-simulator of patients with non-small cell lung cancer on dose limiting normal tissues and radiation dose-escalation: a planning study. Radiother Oncol 2005;77:5-10.

28. De Ruysscher D, Wanders S, van Haren E, et al. Selective mediastinal node irradiation based on FDG-PET scan data in patients with non-small-cell lung cancer: a prospective clinical study. Int J Radiat Oncol Biol Phys 2005;62:988-994.

29. Dizendorf EV, Baumert BG, von Schulthess GK, et al. Impact of whole-body 18F-FDG PET on staging and managing patients for radiation therapy. J Nucl Med 2003;44:24-29.

30. Kalff V, Hicks RJ, MacManus MP, et al. Clinical impact of (18)F fluorodeoxyglucose positron emission tomography in patients with non-small-cell lung cancer: a prospective study. J Clin Oncol 2001;19:111-118.

31. Caldwell $\mathrm{CB}$, Mah K, Ung YC, et al. Observer variation in contouring gross tumor volume in patients with poorly defined non-small-cell lung tumors on CT: the impact of 18FDG-hybrid PET fusion. Int J Radiat Oncol Biol Phys 2001;51:923-931.

32. Fox JL, Rengan R, O'Meara W, et al. Does registration of PET and planning CT images decrease interobserver and intraobserver variation in delineating tumor volumes for non-small-cell lung cancer? Int J Radiat Oncol Biol Phys 2005;62:70-75.

33. Steenbakkers RJ, Duppen JC, Fitton I, et al. Reduction of observer variation using matched CTPET for lung cancer delineation: a three-dimensional analysis. Int $\mathrm{J}$ Radiat Oncol Biol Phys 2006;64:435-448.

34. Ciernik IF, Huser M, Burger $C$, et al. Automated functional image-guided radiation treatment planning for rectal cancer. Int J Radiat Oncol Biol Phys 2005;62:893-900.

35. Daisne JF, Sibomana M, Bol A, et al. Tri-dimensional automatic segmentation of PET volumes based on measured source-to-background ratios: influence of reconstruction algorithms. Radiother Oncol 2003;69:247-250.

36. Erdi YE, Mawlawi O, Larson SM, et al. Segmentation of lung lesion volume by adaptive positron emission tomography image thresholding. Cancer 1997;80:2505-2509.

37. Nestle U, Kremp S, Schaefer-Schuler A, et al. Comparison of different methods for delineation of 18F-FDG PET-positive tissue for target volume definition in radiotherapy of patients with nonSmall cell lung cancer. J Nucl Med 2005;46:1342-1348.

38. Daisne JF, Duprez T, Weynand B, et al. Tumor volume in pharyngolaryngeal squamous cell carcinoma: comparison at CT, MR imaging, and FDG PET and validation with surgical specimen. Radiology 2004;233:93-100. 
39. Thie JA. Understanding the standardized uptake value, its methods, and implications for usage. J Nucl Med 2004;45:1431-1434.

40. De Leyn P, Stroobants S, De Wever W, et al. Prospective comparative study of integrated positron emission tomography-computed tomography scan compared with remediastinoscopy in the assessment of residual mediastinal lymph node disease after induction chemotherapy for mediastinoscopy-proven stage IIIA-N2 Non-small-cell lung cancer: a Leuven Lung Cancer Group Study. J Clin Oncol 2006;24:3333-3339.

41. Giraud $\mathrm{P}$, Elles $\mathrm{S}$, Helfre $\mathrm{S}$, et al. Conformal radiotherapy for lung cancer: different delineation of the gross tumor volume (GTV) by radiologists and radiation oncologists. Radiother Oncol 2002;62:27-36.

42. Hanley JA, McNeil BJ. A method of comparing the areas under receiver operating characteristic curves derived from the same cases. Radiology 1983;148:839-843.

43. Bentzen SM. High-tech in radiation oncology: should there be a ceiling? Int J Radiat Oncol Biol Phys 2004;58:320-330.

44. Loo BW, Quon A, Vasanawala MS, et al. A method of target definition in PET-based radiotherapy planning. Int J Radiat Oncol Biol Phys 2004;60:S602.

45. Birim O, Kappetein AP, Stijnen $T$, et al. Meta-analysis of positron emission tomographic and computed tomographic imaging in detecting mediastinal lymph node metastases in nonsmall cell lung cancer. Ann Thorac Surg 2005;79:375-382.

46. Luketich JD, Friedman DM, Meltzer CC, et al. The role of positron emission tomography in evaluating mediastinal lymph node metastases in non-small-cell lung cancer. Clin Lung Cancer $2001 ; 2: 229-233$.

47. Vanuytsel L, Vansteenkiste JF, Stroobants SG, et al. The impact of (18)F-fluoro-2-deoxy-Dglucose positron emission tomography (FDG-PET) lymph node staging on the radiation treatment volumes in patients with non-small cell lung cancer. Radiother Oncol 2000;55:317-324.

48. Stroom J, Blaauwgeers $\mathrm{H}$, Belderbos J, et al. Feasibility of pathology-correlated lung imaging for accurate target definition of lung tumors. 8th Biennial ESTRO meeting on physics and Radiation Technology for Clinical Radiotherapy - Lisbon 2005: S79 



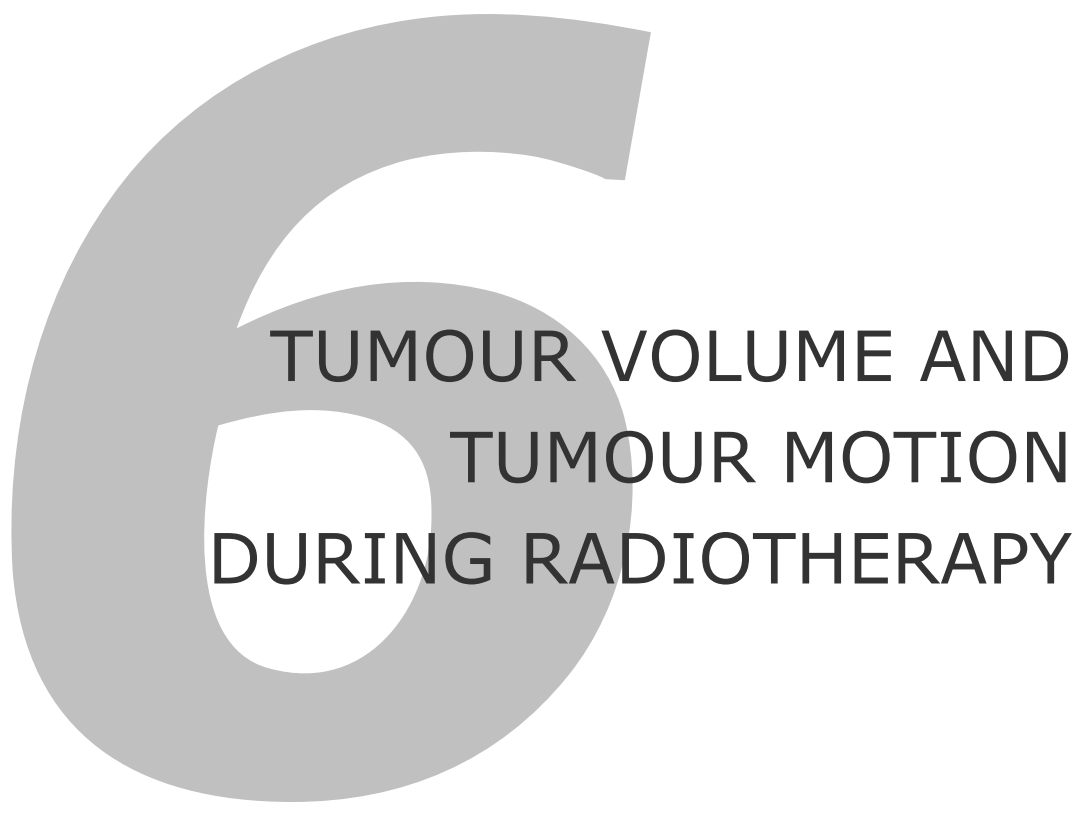

G. Bosmans, A. van Baardwijk, A. Dekker, M. Öllers, L. Boersma, A. Minken, P. Lambin and $D$. De Ruysscher. Intra-patient variability of tumor volume and tumor motion during conventionally fractionated radiotherapy for locally advanced non-small cell lung cancer. A Prospective Clinical Study.

Int J Rad Oncol Biol Phys 2006;66(3):748-53 


\section{Abstract}

Purpose: To investigate the change in tumor volume, motion and breathing frequency during a course of radiotherapy, for locally advanced non-small cell lung cancer.

Materials and Methods: Twenty-three patients underwent CT-PET and Respiration Correlated CT scans prior to treatment, which was repeated in the first and second week following the start of radiotherapy. Patients were treated with an accelerated fractionation schedule, 1.8 Gy BID, with a total tumor dose depending on pre-set dose constraints for the lungs and the spinal cord.

Results: A striking heterogeneity of tumor volume changes was observed at all time points. In some patients the volume decreased $>30 \%(3 / 23)$ in others the volume increased $>30 \%(4 / 24)$ but for the majority of patients $(16 / 23)$ the tumor volume only changed slightly $(<30 \%)$. No significant changes in average tumor motion or breathing frequencies were seen during treatment. Although quite some changes in individual tumor motion were seen, only in one patient would this have led to an increase of the internal margin $>1 \mathrm{~mm}$ in one direction, one week after the start of treatment, and in three patients for one direction, 2 weeks after the start of the treatment.

Conclusions: A large variability of changes in tumor volume between the patients was observed. This underscores the need for repeated imaging during the course of radiotherapy. However, the changes in tumor motion are small, which indicates that repeated respiration correlated CT does not appear to be necessary. 


\section{Introduction}

Radiation therapy, alone or in combination with chemotherapy or surgery, is important in the treatment of lung ${ }^{1}$. Nowadays, target volume delineation is often based on sophisticated imaging techniques such as CT and FDG-PET scans, as this improves the diagnostic accuracy and may allow radiation dose escalation ${ }^{2-5}$. Breathing induced tumor motion and hence appropriate margins can be taken into account using 4D CT information from respiration correlated CT (RC-CT) scans. Incorporating this temporal information in radiotherapy planning could also lead to a more accurate delineation of the gross tumor volume. In general, smaller margins can be applied which would make dose escalation or a decrease of normal tissue exposure possible 6 . However, radiation treatment planning is based on imaging taken at a single time-point before the start of radiotherapy, and therefore any changes of tumor motion, tumor volume or breathing frequency during the course of fractionated radiotherapy are not incorporated in the treatment planning process.

Few data on volume changes during radiotherapy for lung cancer patients have been reported $^{7-10}$, showing conflicting results. Moreover, the evolution of the tumor motion during radiotherapy was only reported in stereotactic radiotherapy for stage I NSCLC patients ${ }^{10}$, although this information is important for Adaptive RadioTherapy (ART). We therefore investigated the changes of tumor volume, tumor motion and breathing frequency during the first 2 weeks of an accelerated course of radiotherapy in patients with locally advanced non-small cell lung cancer (NSCLC).

\section{Materials and Methods}

\section{Patients}

Twenty-three patients with histological or cytological proven non-small cell lung cancer (NSCLC) (UICC stage II-III) were included in this study, from December 2004 until September 2005. Twenty patients received three cycles of Carboplatin and Gemcitabine chemotherapy before radiotherapy, had a WHO performance status $0-1$ and a good lung function $\left(\mathrm{FEV}_{1} \geq 50 \%\right.$ and $\mathrm{DLCO} \geq 50 \%$ ). Three patients did not receive chemotherapy due to medical reasons. The response to induction chemotherapy was evaluated using the RECIST-criteria ${ }^{11}$. No concurrent chemotherapy was given. Patients were treated using our current standard CT-PET simulation, radiation treatment planning, and fractionation schedule, as described below.

The Medical Ethics Committee according to the Dutch law approved the trial. All patients gave written informed consent before entering this study.

\section{Radiotherapy simulation}

Patients were simulated in radiotherapy position on a dedicated CT-PET-simulator with both arms above the head and on an immobilization and patient laser marker system. Radiotherapy technologists trained specifically for this purpose always carried out the simulation. The CT-PET scanner used was a Siemens Biograph ${ }^{\mathrm{TM}}$ (SOMATOM $^{\circledR}$ Sensation 16 with an ECAT ACCEL PET scanner). An intravenous injection of ${ }^{18}$ F-Fluoro- 
Deoxy-Glucose (FDG) (Tyco Health Care, Amsterdam, The Netherlands) of (weight*4+20) MBq was followed by $10 \mathrm{ml}$ physiologic saline. After a 45 minutes uptake period, during which the patient was encouraged to rest, PET and CT images were acquired. A spiral CT with intravenous contrast was performed covering the complete thoracic region.

\section{Radiotherapy planning}

Radiotherapy planning was performed on a XiO (Computerized Medical Systems, St Louis, Missouri) treatment planning system, using inhomogeneity corrections, based on a convolution algorithm. For all patients, the Gross Tumor Volume (GTV) and the Planning Target Volume (PTV) were defined, based on CT-PET data, obtained prior to radiotherapy (i.e. post chemotherapy in case of induction chemotherapy). The Clinical Target Volume (CTV) was defined as the GTV with a $5 \mathrm{~mm}$ margin incorporating microscopic disease. This CTV was subsequently expanded with $1 \mathrm{~cm}$ to draw the PTV to incorporate the internal respiratory motion and setup errors. For the volume comparison in this study, the CT-GTV was defined as the primary tumor on lung window (W 1600, L -800). Contouring of the lungs was carried out automatically by the treatment planning system. For the calculation of the mean lung dose (MLD), the volume of both lungs excluding the GTV was used ${ }^{12}$. The spinal cord was drawn throughout the whole CT scan and was considered to be at the inner margin of the bony spinal canal. A 3D conformal treatment plan was calculated on the PTV for all patients according to ICRU 50 guidelines ${ }^{13}$. Dosimetric values were calculated on the basis of dose-volume histograms and dose distributions on each axial CT plan.

\section{Radiation dose and normal tissue constraints}

For each patient, the radiation dose was escalated to a mean lung dose (MLD) of $19 \mathrm{~Gy}$ while respecting a maximum spinal cord dose of $54 \mathrm{~Gy}$. There were no esophageal dose constraints. As the tumor dose was delivered in $1.8 \mathrm{~Gy}$ fractions, a maximum deviation of the constraints of $\pm 4 \%$ was allowed. Radiotherapy was always delivered twice a day (BID), with a minimum of 8 hours between the two fractions, and given 5 days per week.

\section{Respiration Correlated CT scans (RC-CT)}

For the patients who gave informed consent for this study, the CT-PET was immediately followed by a respiration correlated CT, using offline correlation of the 16 slice spiral CT scan of the CT-PET system. This had been modified to enable a low pitch (minimum 0.1), in combination with a respiratory signal obtained by a pressure sensor in a chest belt (AZ-733V, Anzai Medical Corporation, Tokyo, Japan). The raw CT data were reconstructed in 10 phases, from $0 \%$ to $100 \%$ in steps of $10 \%$ of the respiration period starting at end inspiration. In-house developed software with Matlab (Mathworks Inc, Natick, MA) was used to measure the motion of the lesion in all three orthogonal directions. 


\section{Study design}

During the course of radiotherapy, the patients underwent a repeat CT-PET scan and a respiration correlated $\mathrm{CT}$ in treatment position, one and two weeks after the start of treatment. At all time points, the primary tumor was delineated using the freebreathing $\mathrm{CT}$ rather than on a single phase of a respiration correlated $\mathrm{CT}$, as respiration correlated CT data was not available in all patients. This approach assumes that no blurring of the moving tumor occurs in the free-breathing CT. This assumption was checked and found to be valid as the high-speed 16 slice CT scan protocol, effectively 'freezes' the tumor in a single phase during a free-breathing СT. The tumor was delineated for each patient by the same physician to avoid inter-observer variability. To study the intra-observer variability, all pre-treatment CT-scans were delineated twice by the same physician, as was the case for ten patients for all time points, with at least a 3 month time interval.

Due to technical and logistical problems, a complete image dataset at all three time points, was not available for all patients. We missed a CT-PET dataset for one patient one week after the start of treatment and for three patients, two weeks after the start of treatment.

\section{Statistics}

We determined the tumor volumes prior to, and 1 and 2 weeks after start of the treatment, based on the GTVs drawn on the free-breathing CT. In addition, we used the respiration correlated CT to quantify the tumor motion at all 3 time points. Tumor motion was expressed as a $3 D$ vector ${ }^{14}$, which is the quadratic sum of the motions in the three orthogonal directions. Finally, breathing frequency was recorded at all three time-points during the respiration correlated CT. All results are expressed as the mean \pm standard deviation (SD). Statistical differences between paired parameters from the three time points were evaluated using the Wilcoxon signed rank test. Differences were considered to be significant when the $p$-value was less than 0.05 .

\section{Results}

\section{Patient characteristics}

The patient characteristics are shown in Table 6.1. The mean values of the total tumor dose (TTD) and the corresponding mean lung dose (MLD) were $61.7 \pm 9.8 \mathrm{~Gy}$ (range 46.8-79.2 Gy) and 16.4 $\pm 3.4 \mathrm{~Gy}$ (range 6.8-20.8 Gy) respectively. The GTV of the primary tumor before radiotherapy was $87.5 \pm 137.7 \mathrm{~cm}^{3}$ (range $0.4-660.2 \mathrm{~cm}^{3}$ ). The first repeat scan was made after the patient received on average a dose of $20.4 \pm 4.1 \mathrm{~Gy}$ (about 11 fractions) and the second scan after 38.3 \pm 5.1 Gy (about 21 fractions; Table $6.2)$. 


\begin{tabular}{|c|c|c|c|c|c|c|c|c|}
\hline Patient no. & $\begin{array}{c}\text { Age } \\
\text { (years) }\end{array}$ & Gender & Stage & $\begin{array}{l}\text { Location of the } \\
\text { primary tumor }\end{array}$ & $\begin{array}{l}\text { Response to } \\
\text { chemotherapy }\end{array}$ & $\begin{array}{l}\text { TTD } \\
\text { (Gy) }\end{array}$ & $\begin{array}{l}\text { MLD } \\
\text { (Gy) }\end{array}$ & $\begin{array}{c}\text { OTT } \\
\text { (days) }\end{array}$ \\
\hline 1 & 52 & $\mathrm{~F}$ & T1N3M0 & RLL & PR & 54.0 & 17.6 & 17 \\
\hline 2 & 54 & $M$ & T3N2MO & RML & SD & 54.0 & 19.3 & 20 \\
\hline 3 & 75 & $M$ & T3N2MO & RUL & PR & 54.0 & 15.2 & 20 \\
\hline 4 & 71 & $\mathrm{~F}$ & T3N1M0 & RUL & no chemo & 64.8 & 20.7 & 23 \\
\hline 5 & 71 & $M$ & T2N3M0 & RUL & PR & 54.0 & 14.7 & 23 \\
\hline 6 & 61 & $M$ & T1N3M0 & RML & PR & 68.4 & 14.9 & 22 \\
\hline 7 & 71 & $M$ & T2N3M0 & LLL & PR & 54.0 & 16.0 & 19 \\
\hline 8 & 77 & $M$ & T4N2M0 & RUL & PR & 61.2 & 17.6 & 22 \\
\hline 9 & 74 & $\mathrm{~F}$ & T2NOMO & RLL & no chemo & 54.0 & 16.3 & 20 \\
\hline 10 & 79 & $\mathrm{~F}$ & T4NOMO & RLL & PR & 57.6 & 18.3 & 23 \\
\hline 11 & 57 & $M$ & T1N3M0 & LUL & PR & 64.8 & 8.5 & 27 \\
\hline 12 & 68 & $M$ & T4NOMO & RUL & PR & 54.0 & 6.8 & 20 \\
\hline 13 & 63 & $M$ & T4NOMO & RLL & SD & 64.8 & 17.3 & 24 \\
\hline 14 & 59 & $\mathrm{~F}$ & $\mathrm{~T} 2 \mathrm{~N} 2 \mathrm{MO}$ & RML & PR & 46.8 & 20.0 & 16 \\
\hline 15 & 55 & $M$ & T4N2M0 & LUL & SD & 79.2 & 16.4 & 29 \\
\hline 16 & 52 & $\mathrm{~F}$ & T2N3M0 & LLL & PR & 54.0 & 15.6 & 20 \\
\hline 17 & 50 & $\mathrm{~F}$ & T4N2MO & LLL & PR & 61.2 & 20.8 & 21 \\
\hline 18 & 71 & M & T4N2M0 & RUL & PR & 79.2 & 13.6 & 29 \\
\hline 19 & 61 & $\mathrm{~F}$ & T2N2MO & RML & $P R$ & 57.6 & 19.0 & 21 \\
\hline 20 & 62 & $M$ & T2N2MO & RUL & $P R$ & 68.4 & 18.8 & 27 \\
\hline 21 & 80 & $M$ & T2NOMO & LLL & no chemo & 61.2 & 15.2 & 21 \\
\hline 22 & 56 & $M$ & T4N2MO & LUL & SD & 79.2 & 17.8 & 30 \\
\hline 23 & 54 & $M$ & T2N3M0 & LUL & SD & 79.2 & 16.1 & 30 \\
\hline Mean & 64.0 & & & & & 61.7 & 16.4 & 22.8 \\
\hline SD & 9.6 & & & & & 9.8 & 3.4 & 4.1 \\
\hline
\end{tabular}

\section{Volume changes of the primary tumour}

A striking heterogeneity of the changes in volume of the primary tumor during radiotherapy was observed. Using an arbitrarily chosen cut-off level of $30 \%$, four patients $(17 \%)$ showed an increase of the tumor volume of $>30 \%$, for sixteen patients $(70 \%)$ the volume change varied $<30 \%$ while for three patients $(13 \%)$ the volume decreased $>30 \%$. In Figure 6.1 these patients are indicated with a dotted line for the patients with a large increase in tumor volume, a dashed line for those patients with a large decrease and a solid line for the rest of the patients. Although the average volumes do not reflect the individual changes, we observed a significant increase in average tumor volume in the patients $(n=22)$ for which data at the first and second time point was available, from $90.9 \pm 139.9 \mathrm{~cm}^{3}$ to $99.2 \pm 130.4 \mathrm{~cm}^{3}$ from the start of radiation to week $1(p=0.049)$. For time point at week 2 , we missed the CT information for three patients. In the remaining 20 patients the tumor decreased on average from $94.7 \pm 146.3 \mathrm{~cm}^{3}$ to $86.4 \pm 109.8 \mathrm{~cm}^{3}$ from the start of radiation to week $2(p=0.940$; Table 6.2 and Figure 6.1). No significant difference in tumor volume changes was observed between patients with stable disease and partial response to induction chemotherapy. For the 43 delineations, which were performed twice, we found an average intra-observer variability of $1.2 \% \pm 4.4 \%$, with a maximum deviation of $9.5 \%$. 


\begin{tabular}{|c|c|c|c|c|c|}
\hline Patient no & $\begin{array}{l}\text { Volume before } \\
\qquad \text { RT }\left(\mathrm{cm}^{3}\right)\end{array}$ & $\begin{array}{l}\text { Corresponding dose after } \\
\text { one week of RT (Gy) }\end{array}$ & $\begin{array}{l}\text { Volume after } \\
\text { one week of } \\
\text { RT }\left(\mathrm{cm}^{3}\right)\end{array}$ & $\begin{array}{l}\text { Corresponding dose after } \\
\text { two weeks of RT (Gy) }\end{array}$ & $\begin{array}{l}\text { Volume after } \\
\text { two weeks of } \\
\text { RT }\left(\mathrm{cm}^{3}\right)\end{array}$ \\
\hline 1 & 7.9 & 19.8 & 4.7 & 37.8 & 5.2 \\
\hline 2 & 660.2 & 18.0 & 580.3 & 34.2 & 455.9 \\
\hline 3 & 53.1 & 18.0 & 65.9 & NA & NA \\
\hline 4 & 26.6 & 19.8 & 14.7 & 37.8 & 2.8 \\
\hline 5 & 97.3 & 18.0 & 155.0 & 36.0 & 134.6 \\
\hline 6 & 7.5 & 19.8 & 4.3 & 34.2 & 5.0 \\
\hline 7 & 28.1 & 16.2 & 43.8 & 37.8 & 36.4 \\
\hline 8 & 3.5 & 19.8 & 5.9 & 41.4 & 6.0 \\
\hline 9 & 64.9 & 27.0 & 35.8 & NA & NA \\
\hline 10 & 12.2 & NA & NA & 36.0 & 10.8 \\
\hline 11 & 0.4 & 19.8 & 0.5 & NA & NA \\
\hline 12 & 63.2 & 23.4 & 64.6 & 37.8 & 72.3 \\
\hline 13 & 67.2 & 19.8 & 88.6 & 48.6 & 42.2 \\
\hline 14 & 272.2 & 23.4 & 323.8 & 41.4 & 288.9 \\
\hline 15 & 92.9 & 19.8 & 104.3 & 37.8 & 84.0 \\
\hline 16 & 79.0 & 21.6 & 99.7 & 39.6 & 83.8 \\
\hline 17 & 92.9 & 19.8 & 153.5 & 37.8 & 121.1 \\
\hline 18 & 7.3 & 21.6 & 6.2 & 32.4 & 6.4 \\
\hline 19 & 101.9 & 16.2 & 131.2 & 41.4 & 80.1 \\
\hline 20 & 24.2 & 19.8 & 28.0 & 37.8 & 26.7 \\
\hline 21 & 107.7 & 14.4 & 114.7 & 27 & 105.3 \\
\hline 22 & 73.0 & 18 & 75.9 & 39.6 & 65.0 \\
\hline 23 & 69.0 & 34.2 & 79.9 & 50.4 & 95.8 \\
\hline Mean & 87.5 & 20.4 & 99.2 & 38.3 & 84.4 \\
\hline SD & 137.7 & 4.1 & 130.4 & 5.1 & 109.8 \\
\hline
\end{tabular}

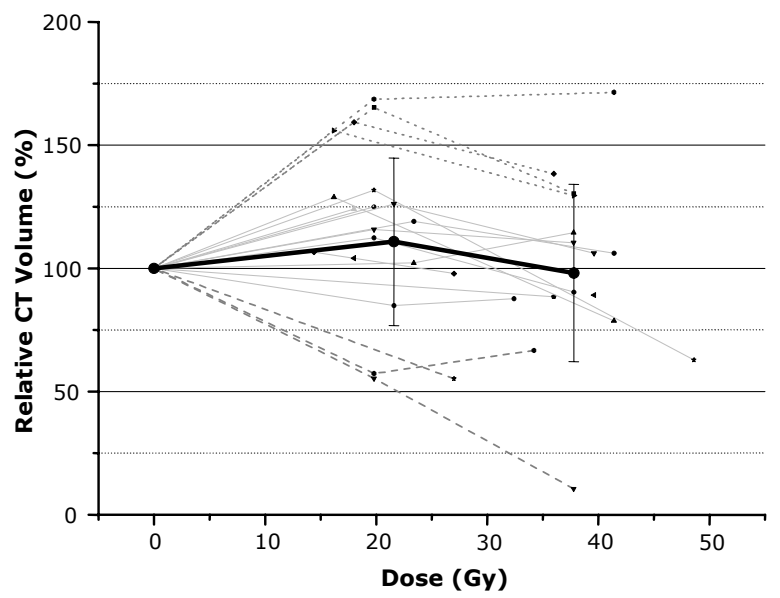

Figure 6.1 The CT volumes, relative to the volume prior to treatment, were plotted versus the dose. The thick black line is the average relative CT volume together with the standard deviation. The dotted lines represents the patients where the tumor increased more than $30 \%$, the dashed lines those patients with a decrease of more than $30 \%$. 


\section{Changes of the motion of the primary tumor}

Tumor motion, expressed as a 3D vector, which is the quadratic sum of the motion in the three orthogonal directions and the breathing frequency are shown in Table 6.3 and Figure 6.2.

On average, the total vector motion changed from $7.8 \pm 4.8 \mathrm{~mm}$ initially to $7.8 \pm 5.9 \mathrm{~mm}$ and $8.0 \pm 5.6 \mathrm{~mm}$ one and two weeks after the start of the radiotherapy respectively. All differences compared with the initial tumor motion were not statistically different from each other ( $p=0.969$ and $p=0.582$ respectively).

\begin{tabular}{|c|c|c|c|c|c|c|}
\hline \multirow[t]{2}{*}{ Patient no } & \multicolumn{2}{|c|}{ Before RT } & \multicolumn{2}{|c|}{ After one week of RT } & \multicolumn{2}{|c|}{ After 2 weeks of RT } \\
\hline & 3D Vector & BPM & 3D Vector & BPM & 3D Vector & BPM \\
\hline 5 & 7.7 & 22.2 & 9.2 & 22.2 & 8.2 & 25.5 \\
\hline 6 & 8.1 & 15.5 & 11.0 & 12.0 & 16.0 & 17.6 \\
\hline 7 & 4.5 & 24.0 & 2.9 & 23.1 & 3.9 & 21.6 \\
\hline 12 & 0.5 & 14.0 & 0.7 & 15.0 & 1.6 & 12.6 \\
\hline 14 & 16.3 & 17.5 & 21.0 & 17.6 & 17.8 & 19.0 \\
\hline 15 & 4.2 & 12.9 & 7.3 & 23.5 & 4.7 & 17.1 \\
\hline 16 & 9.5 & 19.2 & 6.2 & 25.5 & 8.2 & 21.1 \\
\hline 17 & 4.2 & 21.6 & 3.1 & 21.6 & 4.0 & 24.5 \\
\hline 19 & 11.4 & 22.2 & 7.4 & 20.3 & 9.8 & 16.1 \\
\hline 20 & 4.5 & 16.9 & 1.6 & 16.3 & 2.5 & 16.7 \\
\hline 21 & 15.6 & 19.7 & 15.8 & 17.9 & 15.1 & 17.9 \\
\hline 22 & 7.0 & 16.0 & 6.9 & 15.4 & 4.6 & 9.9 \\
\hline Mean & 7.8 & 18.5 & 7.8 & 19.2 & 8.0 & 18.3 \\
\hline SD & 4.8 & 3.6 & 5.9 & 4.1 & 5.6 & 4.5 \\
\hline
\end{tabular}

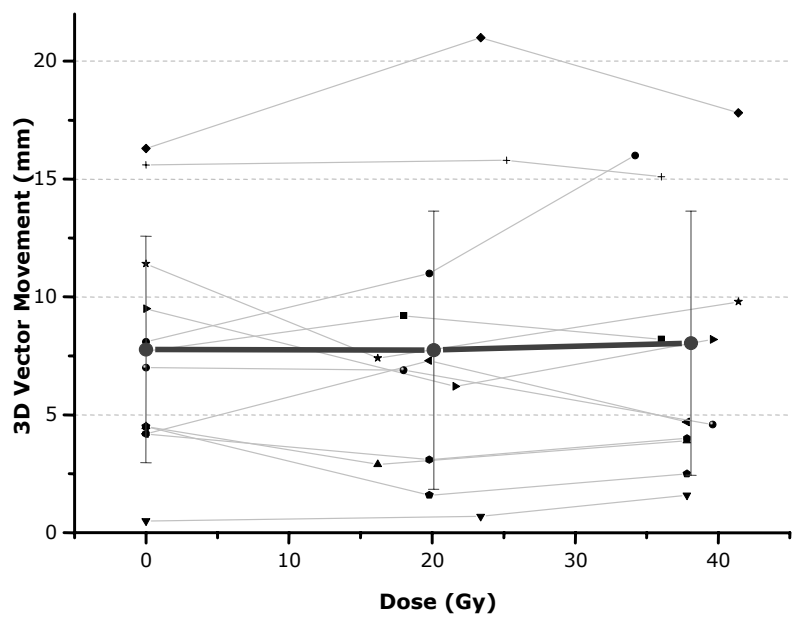

Figure $6.23 D$-Vector motion, which is the quadratic sum of the motion in three orthogonal directions, plotted versus the dose. The thick black line represents the average tumor motion, together with the standard deviation. 
When we looked at the individual differences for the motion for all directions compared with the initial motion, we observed a maximal absolute difference of $4.5 \mathrm{~mm}$ and $6.4 \mathrm{~mm}$ one and two weeks after the start of radiotherapy. The maximal difference between week 2 and week 1 after the start of radiotherapy was $5.4 \mathrm{~mm}$. In Figure 6.3 a histogram of these differences is shown.

If we use the peak to peak amplitude divided by four, as used for the internal margin in Van Herk's margin recipe ${ }^{15}$, only for one patient, for one direction the internal margin increased more than $1 \mathrm{~mm}$ one week after the start of radiotherapy. Two weeks after radiotherapy the internal margin should have been changed for three patients with more than $1 \mathrm{~mm}$ (Figure 6.3).
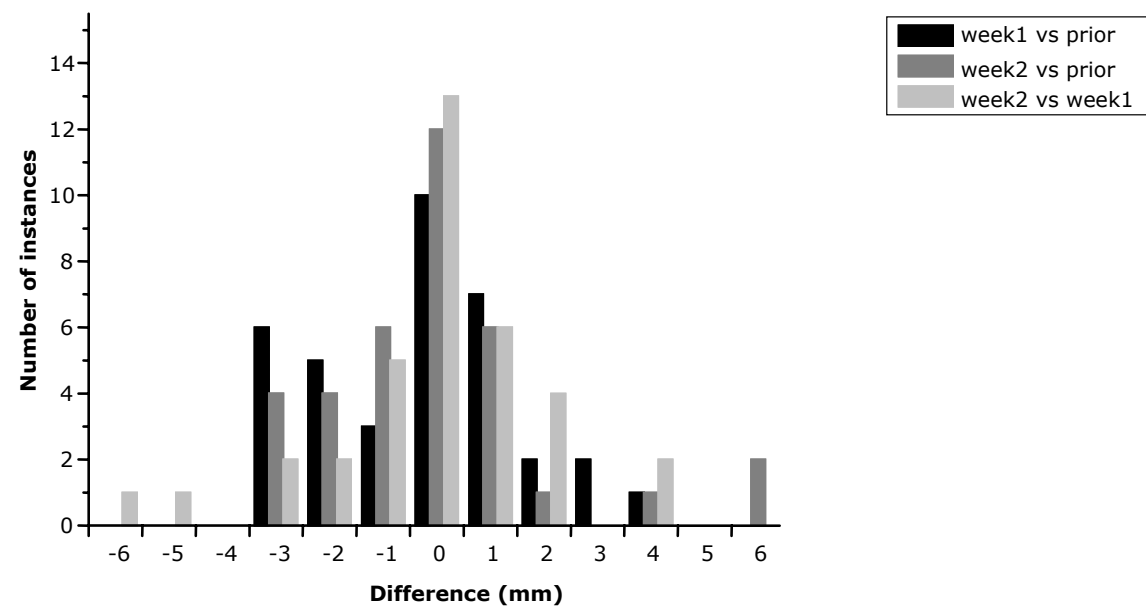

Figure 6.3 Histogram of the differences $(\mathrm{mm})$ in motion amplitude between all time points. The differences for all three orthogonal directions are shown.

\section{Change of the breathing frequency}

The breathing frequency was not statistically different at the three time points. On average, the frequency changed from $18.5 \pm 3.6$ breathing cycles per minute (BPM) to $19.2 \pm 4.1$ BPM and $18.3 \pm 4.5$ BPM ( $p=0.80$ and $p=0.88$ respectively).

\section{Discussion}

For high precision radiation therapy, it is important to have, during a course of radiation therapy, knowledge of the changes of several parameters, such as the tumor volume and tumor motion. We observed a large heterogeneity of the changes in tumor volume in our patient population. Only a few patients $(3 / 23)$ showed a large systematic decrease $(>30 \%)$ in tumor volume, whereas in the majority of patients $(16 / 23)$, the tumor volume only slightly changed $(<30 \%)$. We even found a large increase in tumor volume for four out of 23 patients. The clinical impact of these volume changes and how to adapt treatment is however still unclear. Further clinical and planning studies need to be done to clarify this problem. 
So, in contrast to other investigator ${ }^{7,8}$, no significant decrease in the tumor volume during the first two weeks of radiotherapy was found. Several differences between the reported studies and our study may explain the differences. In contrast to the present study, in the studies of Kupelian et al. ${ }^{8}$ and Erridge et al. ${ }^{7}$, patients were treated with a conventional fractionation scheme ( 2 Gy/day) delivered over an overall treatment time of more than 40 days. In our study, all patients received an accelerated schedule in which 1.8 Gy per fraction was delivered BID, 5 days per week. It may be that the decrease of the tumor volume by radiation-induced cancer cell death was offset by treatment-induced inflammation, which once again increased the volume. The net result may be a stable volume. Comparable results in tumor volume changes on CT scans in the first two weeks due to radiotherapy have been described when high doses per fractions (7.5-20 Gy) are used in stereotactic radiotherapy for stage I NSCLC ${ }^{9,10}$. We may get more insight in the internal tumor processes by analyzing the FDG distribution in the tumor during the course of radiation. Unfortunately it is still too early to correlate these changes in tumor volume with outcome. These analyses will however be the subject of another paper.

To the best of our knowledge, this is the first study dealing with the changes of the motion and the breathing frequency of patients with locally advanced (Stage II-III) NSCLC during a course of radiotherapy. Underberg et al. ${ }^{10}$ described similar findings for Stage I NSCLC treated with hypofractionated stereotactic radiotherapy. Although we found no significant changes, on average, in tumor motion and breathing frequency, during the first two weeks of radiotherapy, in some patients quite a wide variation was seen. However, even for the largest difference in tumor motion, the clinical implication on the size of the PTV and hence on the treatment fields is limited, since only a fraction of this motion is used for PTV expansion to account for the tumor motion. Only for one patient, for one direction the internal margin increased more than $1 \mathrm{~mm}$ one week after the start of radiotherapy. Two weeks after radiotherapy the internal margin should have been changed for three patients with more than $1 \mathrm{~mm}$ (Figure 6.3). In this patient group, the respiration correlated CT information will not influence the internal margin that needs to be taken.

In conclusion, our results clearly show that, at least when an accelerated radiotherapy schedule is given, changes in the volume of the primary tumor do occur during the first two weeks of radiotherapy. A large variability among the patients was observed, indicating that repeated imaging during the course of radiotherapy is necessary to know the changes in GTV and to be able to individualize radiation treatment. In contrast, repeated respiration correlated CT imaging during radiotherapy does not appear to be necessary as the changes in tumor motion are small, and will not lead to a change in treatment. 


\section{References}

1. De Ruysscher D, Wanders R, Boersma M, et al. Radiotherapy in the management of lung cancer. International Journal of Respiratory Care 2005:1-5.

2. Bradley J, Graham MV, Winter K, et al. Toxicity and outcome results of RTOG 9311: a phase III dose-escalation study using three-dimensional conformal radiotherapy in patients with inoperable non-small-cell lung carcinoma. Int J Radiat Oncol Biol Phys 2005;61:318-328.

3. De Ruysscher D, Wanders S, Minken A, et al. Effects of radiotherapy planning with a dedicated combined PET-CT-simulator of patients with non-small cell lung cancer on dose limiting normal tissues and radiation dose-escalation: a planning study. Radiother Oncol 2005;77:5-10.

4. De Ruysscher D, Wanders S, van Haren E, et al. Selective mediastinal node irradiation based on FDG-PET scan data in patients with non-small-cell lung cancer: a prospective clinical study. Int J Radiat Oncol Biol Phys 2005;62:988-994.

5. van Der Wel A, Nijsten S, Hochstenbag $M$, et al. Increased therapeutic ratio by 18FDG-PET CT planning in patients with clinical CT stage N2-N3M0 non-small-cell lung cancer: a modeling study. Int J Radiat Oncol Biol Phys 2005;61:649-655.

6. Bosmans G, Buijsen J, Dekker A, et al. An "in silico" clinical trial comparing free breathing, slow and respiration correlated computed tomography in lung cancer patients. Radiother Oncol 2006;81:73-80.

7. Erridge SC, Seppenwoolde $\mathrm{Y}$, Muller SH, et al. Portal imaging to assess set-up errors, tumor motion and tumor shrinkage during conformal radiotherapy of non-small cell lung cancer. Radiother Oncol 2003;66:75-85.

8. Kupelian PA, Ramsey C, Meeks SL, et al. Serial megavoltage CT imaging during external beam radiotherapy for non-small-cell lung cancer: observations on tumor regression during treatment. Int J Radiat Oncol Biol Phys 2005;63:1024-1028.

9. Senan S, Underberg R, Slotman B, et al. Time trends in target volumes during stereotactic radiotherapy for Stage I non-small cell lung cancer (NSCLC). Int $\mathrm{J}$ Radiat Oncol Biol Phys 2004;60:S281-282.

10. Underberg RW, Lagerwaard FJ, van Tinteren $\mathrm{H}$, et al. Time trends in target volumes for stage I non-small-cell lung cancer after stereotactic radiotherapy. Int $\mathrm{J}$ Radiat Oncol Biol Phys 2006;64:1221-1228.

11. Therasse P, Arbuck SG, Eisenhauer EA, et al. New guidelines to evaluate the response to treatment in solid tumors. European Organization for Research and Treatment of Cancer, National Cancer Institute of the United States, National Cancer Institute of Canada. J Natl Cancer Inst 2000;92:205-216.

12. Kwa SL, Lebesque JV, Theuws JC, et al. Radiation pneumonitis as a function of mean lung dose: an analysis of pooled data of 540 patients. Int J Radiat Oncol Biol Phys 1998;42:1-9.

13. ICRU. Prescribing, recording, and reporting photon beam therapy (Report 62, supplement to ICRU report 50): Bethesda, MD; 1999.

14. van Sornsen de Koste JR, Lagerwaard FJ, Nijssen-Visser MR, et al. Tumor location cannot predict the mobility of lung tumors: a 3D analysis of data generated from multiple CT scans. Int J Radiat Oncol Biol Phys 2003;56:348-354.

15. van Herk M. Errors and margins in radiotherapy. Semin Radiat Oncol 2004;14:52-64. 



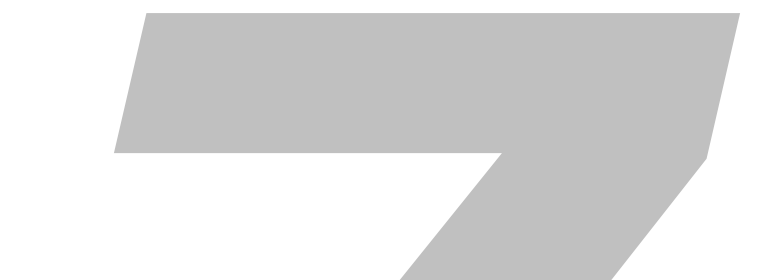

\section{TIME TRENDS IN FDG UPTAKE} DURING RADIOTHERAPY

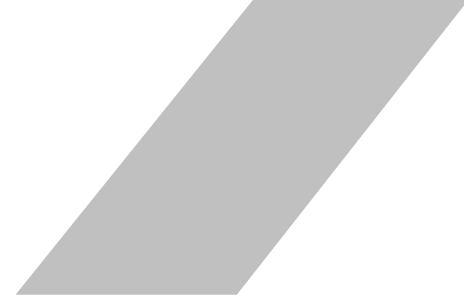

A. van Baardwijk*, G. Bosmans*, A. Dekker, M. van Kroonenburgh, L. Boersma, R. Wanders, M. Öllers, R. Houben, A. Minken, P. Lambin and D. De Ruysscher. Time trends in the maximal uptake of FDG on PET scan during thoracic radiotherapy in relation to metabolic response. A prospective study in locally advanced non-small cell lung cancer (NSCLC) patients. (*these authors equally contributed to this article)

Radiother Oncol 2007;82(2):145-52 


\section{Abstract}

Purpose: ${ }^{18} \mathrm{~F}$-fluoro-2-deoxy-glucose (FDG) uptake on PET scan is a prognostic factor for outcome in NSCLC. We investigated changes in FDG uptake during fractionated radiotherapy in relation to metabolic response with the ultimate aim to adapt treatment according to early response.

Materials and Methods: Twenty-three patients, medically inoperable or with advanced NSCLC, underwent four repeated PET-CT scans before, during and after radiotherapy. Changes in maximal Standardized Uptake Value $\left(S U V_{\max }\right)$ were described. Patients were treated with accelerated radiotherapy with a total tumour dose depending on normal tissue dose constraints.

Results: The most striking result was the large intra-individual heterogeneity in the evolution of $S V_{\max }$. For the total group a non-significant increase in the first week $(p=0.052)$, a decrease in the second week $(p=0.023)$ and after radiotherapy $(p=0.005)$ was observed. Different time trends were shown for responders (no change during radiotherapy) and non-responders (48\% increase during first week, $p=0.023$ and $15 \%$ decrease in the second week, $\mathrm{p}=0.042)$. Non-responders had a higher $\mathrm{SUV}_{\max }$ on all time points investigated.

Conclusions: Time trends in SUV $\mathrm{Vax}_{\text {max }}$ showed a large intra-individual heterogeneity and different patterns for metabolic responders and non-responders. These new findings may reflect intrinsic tumour characteristics and might finally be useful to adapt treatment. 


\section{Introduction}

Thoracic irradiation plays an important role in the management of advanced non-small cell lung cancer (NSCLC). Although the prognosis of these patients has improved, local tumour control is still not achieved in the majority of the patients ${ }^{1-3}$. Local tumour control can be improved by increasing the total radiation dose and the addition of chemotherapy to radiotherapy, but normal tissue toxicity, like esophagitis and radiation pneumonitis, is dose limiting ${ }^{4-9}$. A more individualized prediction of response to a specific therapy is needed in order to avoid needless treatment or to be able to propose an alternative therapy if possible ${ }^{10-12}$. Methods to describe tumour characteristics include imaging techniques, like positron emission tomography (PET). In different tumour sites pre- and post treatment PET scans have been correlated to outcome ${ }^{13-15}$. Indeed, the maximal uptake of ${ }^{18} \mathrm{~F}$-fluoro-2-deoxy-glucose (FDG) in the primary lung tumour as measured on a PET-scan before treatment was consistently shown to be a significant, independent, prognostic factor for survival in NSCLC, both in case of surgical resection and radical radiotherapy ${ }^{16-21}$. On the other hand a post-treatment PET scan can predict residual disease and hence outcome in NSCLC ${ }^{22-26}$.

Although there is a paucity on data dealing with the evolution of FDG uptake during fractionated radiotherapy, it is logic to study these time trends as an early predictive parameter, as has been shown for chemotherapy ${ }^{26-30}$. We hypothesized that changes in uptake might reflect early tumour response during radiotherapy, which might finally allow further individualization and adaptation of treatment before therapy is completed $^{31}$.

The aim of the current study was to describe changes in the uptake of FDG on PET scans during a course of radiotherapy in patients with NSCLC and to investigate whether there are differences in time trends in FDG uptake between metabolic responders and non-responders.

\section{Materials and Methods}

\section{Study population}

From December 2004 to September 2005, 23 patients with medically inoperable or locally advanced NSCLC were enrolled in a phase I study. Patients with localized, histologically confirmed NSCLC, a WHO-performance sore of 0 or 1 , a weight loss of $<10 \%$ in 6 months and a reasonable lung function (a $\mathrm{FEV}_{1}>50 \%$ of predicted value and a DLCO $\geq 50 \%$ ) were eligible. In case of previous chemotherapy, radiotherapy was started after a minimum of 14 days after the last chemotherapy course. The response to induction chemotherapy was evaluated using the RECIST-criteria ${ }^{32}$. No previous radiotherapy to the chest was allowed. Patients were not eligible if they had any other concomitant malignant disease, a recent severe cardiac disease or active peptic esophagitis.

PET-CT-scan

As part of this phase I study repeated PET-CT scans (before start of radiotherapy, on day 7 and day 14 during radiotherapy and 70 days after radiotherapy) were performed 
in radiotherapy position on a dedicated PET-CT-simulator with both arms above the head and using a laser marker system. The PET-CT scanner used was a Biograph ${ }^{\mathrm{TM}}$ (SOMATOM $^{\circledR}$ Sensation 16 with its ECAT ACCEL PET scanner, Siemens). Patients were fasting for at least 6 hours before the examination. FDG (MDS Nordion, Liège, Belgium) was injected intravenously and followed by physiologic saline $(10 \mathrm{ml})$. The injected total activity of FDG depended on the weight of the patient: (weight*4+20) MBq. After a rest period of 45 minutes (time needed for uptake of FDG) PET and CT images were acquired. The CT-scan performed was a spiral CT-scan of the whole thorax, with intravenous contrast. The PET images were acquired in 5-minute bed positions, while 2 or 3 positions were needed to scan the whole thoracic region. The Standardized Uptake Value (SUV) was used to measure the amount of uptake of FDG in a selected Region of Interest (ROI). For the determination of the SUV of the primary tumour, a ROI was drawn on the 3D-images in e.soft (version 5.0.24.5, Siemens, Erlangen, Germany) around the region of the primary tumour. SUV was calculated as activity concentration of FDG uptake divided by injected dose/body weight ${ }^{33}$. To avoid partial volume effects as much as possible, the maximal SUV $\left(\mathrm{SUV}_{\max }\right)$ within this ROI was calculated and used for correlations.

\section{Treatment description}

All patients enrolled in this phase I study were irradiated using an individualized prescribed maximal tolerated dose (MTD). This total tumour dose (TTD) was based on normal tissue constraints instead of a classic fixed prescribed dose. For the lungs a mean lung dose (MLD) of $19.0 \mathrm{~Gy}$ and for the spinal cord a maximal dose of $54.0 \mathrm{~Gy}$ were used as dose constraints for normal tissues. The maximal allowed TTD was 79.2 Gy or lower if any of the normal dose constraints were reached. The margin from GTV (gross tumour volume) to CTV (clinical target volume) was $5 \mathrm{~mm}$ and for the PTV (planning target volume) another $10 \mathrm{~mm}$ for the primary tumour and $5 \mathrm{~mm}$ for lymph nodes was added. No elective nodal irradiation was carried out. A 3D conformal treatment plan, using three to six coplanar $10 \mathrm{MV}$ photon fields, was calculated according to the ICRU guidelines ${ }^{34}$. Calculations were performed using an XiO treatment planning system (Computer Medical System, Inc.) with a FFT convolution algorithm for inhomogeneity corrections ${ }^{35}$. Patients were irradiated on a linear accelerator (Elekta SL 15, Crawley, United Kingdom). Radiotherapy was delivered twice daily, with at least an 8-hour interval (BID scheme) and with a fraction size of $1.8 \mathrm{~Gy}$. Results concerning toxicity and outcome of this phase I study will be reported separately.

\section{Assessment of metabolic response}

Metabolic response was measured 70 days after radiotherapy by means of a PET-CT scan. This scan was compared with the pre-treatment scan, following the recommendations of the EORTC (Table 7.1) ${ }^{36}$. The total group was divided in responders, showing complete metabolic response (CMR) or partial metabolic response (PMR) and non-responders with stable metabolic disease (SMD) or progressive metabolic disease (PMD). 
Table 7.1 Definition of metabolic response on PET-CT scans 70 days after radiotherapy.

EORTC criteria

CMR Complete resolution of FDG uptake in tumor, not distinguishable from surrounding tissue

PMR Reduction of more than $25 \%$ in SUV

SMD Changes in SUV of less than $25 \%$

PMD Increase of SUV of more than $25 \%$ or new (metastatic) lesions

Abbreviations: EORTC=European Organization for Research and Treatment of Cancer; CMR=complete metabolic response; $P M R=$ partial metabolic response; $S M D=$ stable metabolic disease; $P M D=$ progressive metabolic disease; SUV $=$ standardized uptake value.

\section{Statistics}

The SPSS software (version 14.0, SPSS Inc., Chicago, IL) was used for statistical analysis. Results are expressed as mean \pm SD (standard deviation) for individual results and as mean \pm SE (standard error of the mean) for groups. The paired student's t-test was used to compare the SUV $\mathrm{Sax}_{\max }$ on different time points, whereas the Mann-Whitney $\mathrm{U}$-test and the Fisher's exact test were used to compare the different groups. Follow-up was updated and frozen at July $1^{\text {st }}$ of 2006 . Survival estimates were calculated using the Kaplan-Meier method and comparison of the survival curves were made using the log rank test. Patients lost to follow-up were censored. Two-tailed p-values are provided and $\mathrm{p}$-values less than 0.05 were considered statistically significant.

\section{Ethics}

This study has been reviewed and approved by the local Medical Ethical Committee, according to the Dutch law and regulations. All patients gave written informed consent before entering this study.

\section{Results}

\section{Patient characteristics}

The patient characteristics are depicted in Table 7.2. Three patients did not receive chemotherapy, while 20 patients received platinum based chemotherapy (Cisplatin or Carboplatin, with Gemcitabin). As a result of induction chemotherapy 15 patients showed a partial remission and 5 patients showed stable disease. The median interval between the last course of chemotherapy and start of radiotherapy was $35 \pm 16$ days (range 14-56 days). The average total tumour dose (TTD) given was 62.5 \pm 9.7 Gy (range $46.8 \mathrm{~Gy}-79.2 \mathrm{~Gy}$ ) in an overall treatment time (OTT) of 22 days (range 17-30 days). The first PET-CT scan was performed before start of radiotherapy (median 9 days before start of radiotherapy, range 4-13 days), the second on day 7 of radiotherapy (at a median dose of $19.8 \pm 4.1 \mathrm{~Gy}$ ) and the third on day 14 of radiotherapy (at a median dose of $37.8 \pm 5.1 \mathrm{~Gy}$ ). The fourth PET-CT scan was performed 70 days after the last day of radiotherapy (median $71 \pm 12$ days). Because of logistic reasons or technical problems with the PET-scan no PET information was available for 1 patient on day 7 and for 4 patients on day 14 of radiotherapy. For 3 patients no PET scan was available after radiotherapy, in one because of technical reasons and in two patients because of early death after radiotherapy due to a myocardial infarction and a pulmonary infection. The median follow-up (FU) from start of RT was 9.2 \pm 3.8 months. 


\begin{tabular}{|c|c|c|c|c|c|c|c|c|}
\hline $\begin{array}{l}\text { patient } \\
\text { no }\end{array}$ & $\begin{array}{c}\text { age } \\
\text { (years) }\end{array}$ & sex & cTNM & pathology & $\begin{array}{l}\text { localization of } \\
\text { primary tumor }\end{array}$ & $\begin{array}{l}\text { induction } \\
\text { chemotherapy }\end{array}$ & $\begin{array}{l}\text { OTT } \\
\text { (days) }\end{array}$ & $\begin{array}{l}\text { TTD } \\
\text { (Gy) }\end{array}$ \\
\hline 1 & 51 & $\mathrm{~F}$ & T1N3M0 & adenoca & RLL & yes & 21 & 54 \\
\hline 2 & 54 & M & T3N2M0 & large cell ca & RML & yes & 21 & 54 \\
\hline 3 & 75 & M & T3N2MO & large cell ca & RUL & yes & 21 & 54 \\
\hline 4 & 71 & $\mathrm{~F}$ & T3N1M0 & scc & RUL & no & 28 & 64.8 \\
\hline 5 & 70 & M & T2N3M0 & adenoca & RUL & yes & 21 & 54 \\
\hline 6 & 61 & M & T1N3M0 & scc & RML & yes & 27 & 68.4 \\
\hline 7 & 71 & M & T2N3M0 & adenoca & LLL & yes & 20 & 60 \\
\hline 8 & 77 & M & T4N2M0 & large cell ca & RUL & yes & 22 & 61.2 \\
\hline 9 & 74 & $\mathrm{~F}$ & T2NOMO & large cell ca & RLL & no & 20 & 54 \\
\hline 10 & 79 & $\mathrm{~F}$ & T4NOMO & large cell ca & RLL & yes & 24 & 57.6 \\
\hline 11 & 57 & M & T1N3M0 & large cell ca & LUL & yes & 30 & 64.8 \\
\hline 12 & 68 & M & T4NOMO & adenoca & RUL & yes & 21 & 54 \\
\hline 13 & 62 & M & T4NOMO & $\mathrm{scc}$ & RLL & yes & 24 & 64.8 \\
\hline 14 & 59 & $\mathrm{~F}$ & $\mathrm{~T} 2 \mathrm{~N} 2 \mathrm{MO}$ & large cell ca & RML & yes & 17 & 46.8 \\
\hline 15 & 55 & $M$ & T4N2MO & adenoca & LUL & yes & 30 & 79.2 \\
\hline 16 & 52 & $\mathrm{~F}$ & T2N3M0 & scc & LLL & yes & 21 & 54 \\
\hline 17 & 54 & $\mathrm{~F}$ & T4N2MO & large cell ca & LLL & yes & 22 & 61.2 \\
\hline 18 & 71 & M & T4N2MO & large cell ca & RUL & yes & 30 & 79.2 \\
\hline 19 & 61 & $\mathrm{~F}$ & $\mathrm{~T} 2 \mathrm{~N} 2 \mathrm{MO}$ & large cell ca & RML & yes & 21 & 57.6 \\
\hline 20 & 62 & M & $\mathrm{T} 2 \mathrm{~N} 2 \mathrm{MO}$ & large cell ca & RUL & yes & 27 & 68.4 \\
\hline 21 & 84 & M & T2NOMO & scc & LLL & no & 22 & 61.2 \\
\hline 22 & 57 & $M$ & T4N2MO & large cell ca & LUL & yes & 30 & 79.2 \\
\hline 23 & 54 & M & T2N3MO & large cell ca & LUL & yes & 30 & 79.2 \\
\hline
\end{tabular}

\section{Time trends in maximal SUV}

There was a large heterogeneity in the changes in $\mathrm{SUV}_{\max }$ of the primary tumour during the radiation treatment between patients (Figure 7.1). Out of the 22 patients who had a PET-CT at day 7 of radiotherapy, 4 patients (18\%) showed a decrease of more than $25 \%$ of the SUV $\max$, whereas $41 \%(9 / 22)$ showed an increase of more than $25 \%$. The remainder $(9 / 22)$ showed a stable SUV $_{\max }(<25 \%$ change) compared with pretreatment values.

For the whole patient group, the $\mathrm{SUV}_{\max }$ was on average $7.3 \pm 0.8$ (range $0.6-16.1$ ) before start of radiotherapy, which increased to $8.9 \pm 1.2$ (range 1.3-23.1) on day 7 of treatment $(p=0.052)$. In the second week, a significant decrease was observed to a $\mathrm{SUV}_{\max }$ of $8.1 \pm 1.0$ (range 2.1-17.8; $\mathrm{p}=0.023$ ). Seventy days after treatment the SUV $_{\max }$ further decreased to a mean level of $4.8 \pm 3.3$ (range 1.2-13.4), a significant decrease compared with day 14 of treatment $(p=0.001)$ and compared with pretreatment levels $(p=0.005$; Figure $7.2 a)$.

For the whole patient group, the $\mathrm{SUV}_{\max }$ was on average $7.3 \pm 0.8$ (range $0.6-16.1$ ) before start of radiotherapy, which increased to $8.9 \pm 1.2$ (range 1.3-23.1) on day 7 of treatment $(p=0.052)$. 


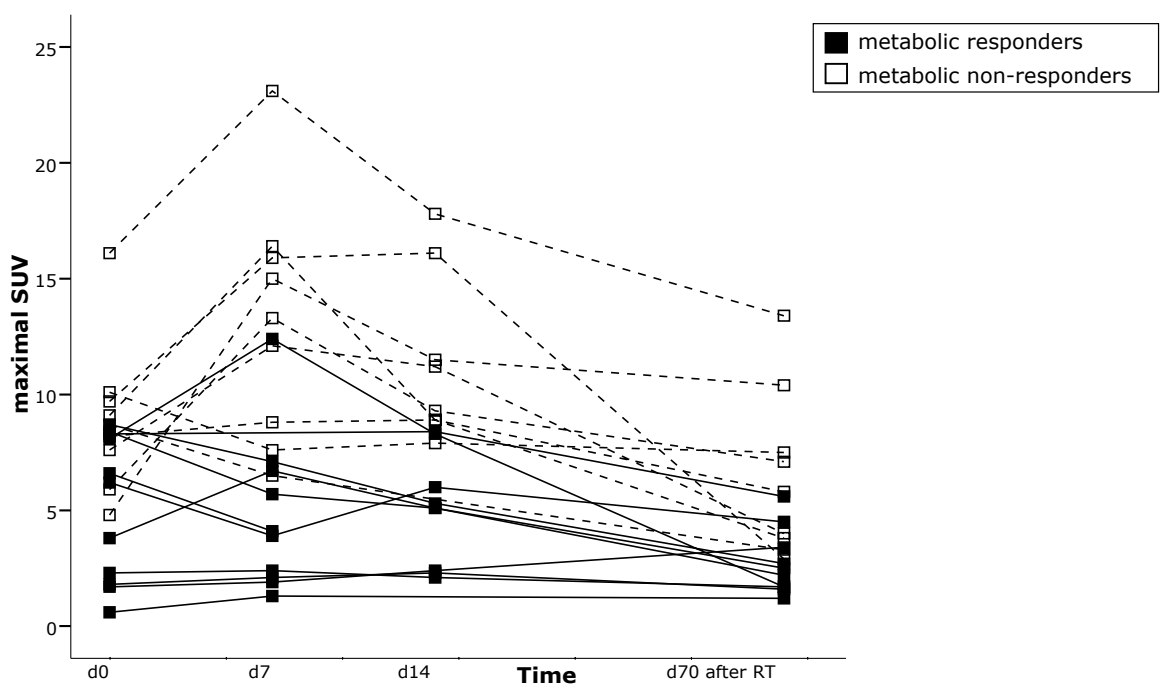

Figure 7.1 The absolute maximal SUV (standardized uptake value) plotted versus time (before RT, day 7 and day 14 during RT and 70 days after RT) for all individual patients with a PET-CT scan at day 70 .

In the second week, a significant decrease was observed to a SUV $\mathrm{max}_{\max }$ of $8.1 \pm 1.0$ (range 2.1-17.8) $(p=0.023)$. Seventy days after treatment the SUV $\max$ further decreased to a mean level of $4.8 \pm 3.3$ (range 1.2-13.4), a significant decrease compared with day 14 of treatment $(p=0.001)$ and compared with pre-treatment levels $(p=0.005 ;$ Figure $7.2 a)$.

Analysing the subset of patients $(n=16)$, who received a PET-CT scan at all time points during radiotherapy, similar results were obtained: SUV $\max$ before radiotherapy $7.0 \pm 0.9$ and at day $79.7 \pm 1.5(p=0.023)$, day $148.0 \pm 1.1(p=0.022)$ and 70 days after radiotherapy $4.7 \pm 0.8(p=0.002)$.

\section{Metabolic responders versus non-responders}

In 20 patients a PET-CT scan was performed 70 days after treatment. In 1 patient (with visually a CMR) no SUV $\max$ could be calculated, due to a software problem. Eleven patients (55\%) showed a metabolic response 70 days after radiotherapy (7 patients CMR and 4 patients PMR), while 9 patients (45\%) showed no response ( 2 patients SMD, 7 patients PMD). Concerning the PMD group, 4 patients had metastatic disease and 3 patients showed loco-regional progression.

Patients with a metabolic response 70 days after thoracic irradiation had a significantly longer overall survival (estimated survival of $100 \%$ after 9 months of follow-up) compared with the patients with stable or progressive disease (estimated survival of $62.5 \%$ after 9 months of follow-up) ( $p=0.005$; Figure 7.3). No difference was observed between total radiation dose prescribed $(p=0.4)$ and the overall treatment time of radiotherapy $(p=0.2)$ between metabolic responders and non-responders. 
In the group of metabolic responders no change in $S_{U} V_{\max }$ was observed during the course of radiotherapy. Only after radiotherapy a mean decrease of $46 \%$ of the SUV $\max$ was observed from $5.0 \pm 0.8$ on day 14 during radiotherapy to $2.7 \pm 0.470$ days after radiotherapy ( $p=0.017$; Figure $2 b$ and Table 3 ). In contrast to this finding, the group of non-responders showed a significant increase of the SUV $\max$ in the first week of radiotherapy. In this group the $S_{U V} V_{\max }$ before treatment was on average $8.9 \pm 1.1$ and increased to $13.2 \pm 1.7$ in the first week $(p=0.023)$. In the second week a decrease was observed to an average level of $11.5 \pm 1.3(p=0.042)$, with a further decrease after radiotherapy to a mean of $6.9 \pm 1.2(p=0.016)$. Similar results were obtained for the subset of patients with a PET-CT scan on day 0, 7 and 14: for the responders SUV max $_{\text {max }}$ did not change during radiotherapy (before radiotherapy: $5.1 \pm 1.1$, day $7: 5.3 \pm 0.8$,

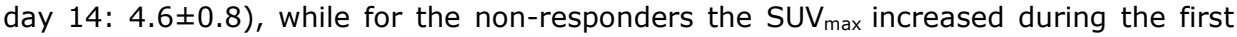
week and decreased again during the second week of radiotherapy (before radiotherapy: $8.9 \pm 1.2$, day $7: 14.0 \pm 1.7(p=0.01)$ and day $1411.5 \pm 1.3(p=0.042)$. Not only a difference in time trends in SUV $\mathrm{Sax}_{\text {mas }}$ waserved between responders and non-responders, but the non-responders also showed a significant higher SUV max $_{\text {than }}$ the responders at all time points investigated (Figure 7.2 and Table 7.3). The number of patients in this study was however too small to test the predictive value of the time trends in SUV $V_{\text {max }}$ in relation to metabolic response.

\section{Influence of induction chemotherapy}

In total 20 patients received induction chemotherapy, of which 18 patients were evaluable 70 days after irradiation. The group of patients achieving a partial remission after induction chemotherapy (13/18) showed in higher percentage metabolic response after radiotherapy (metabolic response rate: $62 \%$ ) as patients showing stable disease as result of the induction chemotherapy (metabolic response rate: $20 \%$ ), although this difference was not significant $(p=0.29)$. The time patterns of the $S U V_{\max }$ in relation to metabolic response as described above for the total group were similar in patients having received induction chemotherapy. This excludes the possibility that the 3 individuals, who did not receive systematic treatment, would have influenced the overall results.

Table 7.3 Maximal SUV (mean \pm SE) before start of radiotherapy, on day 7 and 14 during radiotherapy and 70 days after radiotherapy.

\begin{tabular}{|lcccccccccr|r|}
\hline & \multicolumn{3}{c}{ Total group } & \multicolumn{3}{c|}{ Metabolic responders } & \multicolumn{7}{c|}{$\begin{array}{c}\text { Metabolic non- } \\
\text { responders }\end{array}$} \\
Maximal SUV & mean & SD & $($ no) & mean & SD & $($ no) & mean & SD & (no) & p-value \\
Before start of RT & 7.3 & 3.8 & $(23)$ & 5.1 & 3.1 & $(11)$ & 8.9 & 3.2 & $(9)$ & 0.020 \\
Day 7 of RT & 8.9 & 5.7 & $(22)$ & 4.8 & 3.4 & $(10)$ & 13.9 & 5.2 & $(9)$ & 0.001 \\
Day 14 of RT & 8.1 & 4.3 & $(19)$ & 5.0 & 2.4 & $(9)$ & 11.5 & 3.6 & $(8)$ & $<0.001$ \\
70 days after RT & 4.5 & 3.2 & $(19)$ & 2.7 & 1.4 & $(10)$ & 6.5 & 3.6 & $(9)$ & 0.002 \\
\hline
\end{tabular}

Results for the total group, for patients showing a metabolic response (CMR or PMR) or no metabolic response (SMD or PMD) on radiotherapy 70 days after irradiation. P-value of Mann-Whitney U-test to compare the group of metabolic responders and non-responders. Abbreviations: $R T=$ radiotherapy; $S D=$ standard deviation; no=number. 


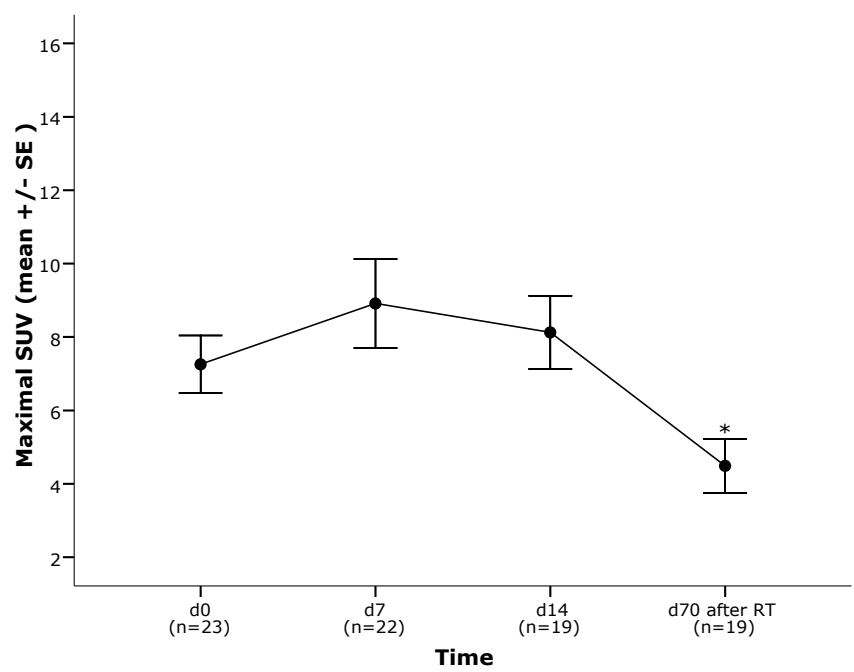

a

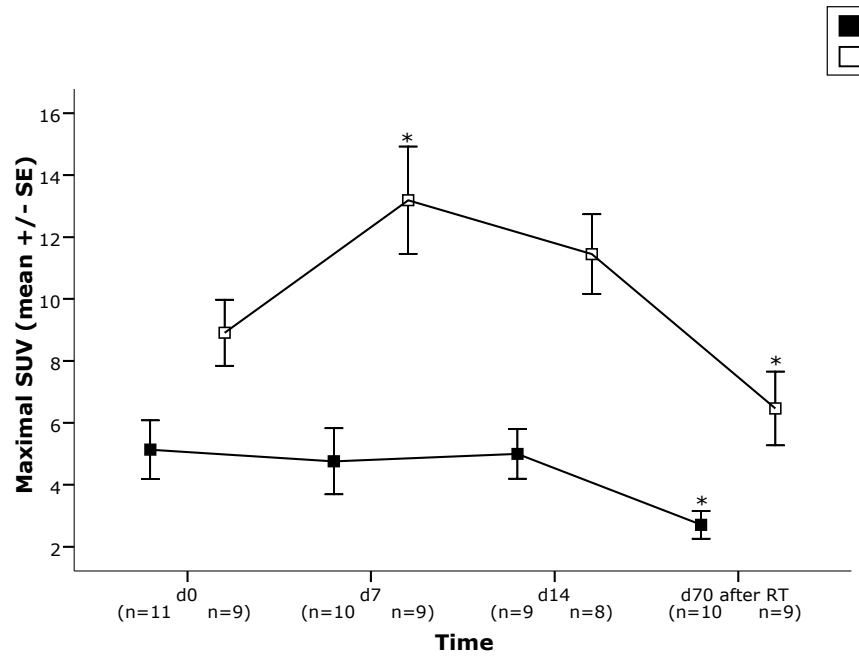

Figure 7.2 Average change in maximal SUV for the total group of patients (a) and for metabolic responders (complete and partial metabolic response) versus non-responders (stable metabolic disease and progressive metabolic disease) (b), *indicates a significant difference. Abrreviations: SUV=standardized uptake value; SE=standard error 


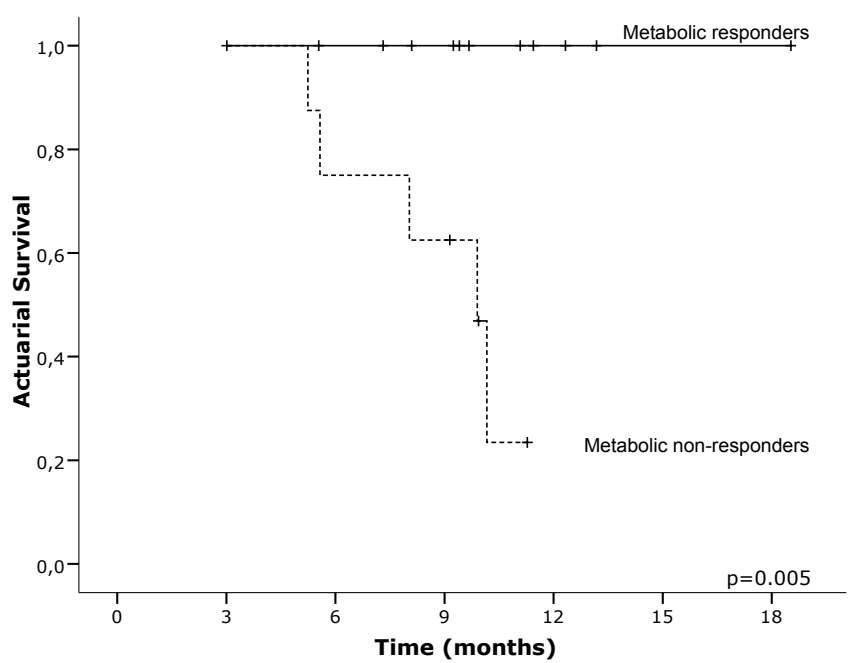

Figure 7.3 Actuarial analysis of overall survival (Kaplan-Meier) in months for metabolic responders $(n=11)$ and non-responders $(n=9)$, classified according to EORTC-criteria. P-value of log-rank test.

\section{Discussion}

Until now the PET-scan is mainly used before start of radiotherapy for staging purposes, to delineate the primary tumour and/or mediastinal lymph nodes and after therapy to assess the remission status $22,25,37-45$. Similar to early response measurements during chemotherapy, FDG-PET scans performed in the beginning of radiotherapy might give information on the later tumour response $26,29,30,46$. However, only limited data on this subject is available in literature ${ }^{27,8}$. We therefore assessed in the present study the time trends of FDG uptake during radiation treatment in NSCLC. A striking result was the large heterogeneity in the SUV $V_{\max }$ in serial PET-scans between the individual patients. While some patients showed an increase, others show a stable or decreased FDG uptake after 1 week of radiation. This confirms the findings of Erdi et al., who described serial PET-scans during radiation therapy in 2 NSCLC patients ${ }^{28}$.

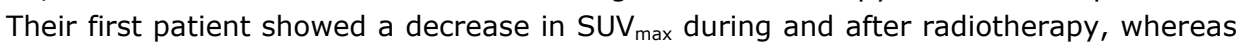
the other patient showed first a decrease followed by an increase in SUV max $_{\text {after }} 40$ days. De Geus-Oei et al. investigated repeated FDG-PET scans in patients treated with ARCON (accelerated radiotherapy with carbogen and nicotinamide) for head-and-neck cancer and also showed very heterogeneous changes in $\mathrm{SUV}_{\max }$ after hyperoxygenation with a median interval of 2 days between the 2 scans $^{27}$.

Investigating the time trends we observed for the total group a trend in increase of the SUV $_{\max }(p=0.052)$ during the first week of radiotherapy, followed by a significant decrease in the second week of treatment and in the first 70 days after treatment. Only few other authors described repeated (CT-)PET scans during radiation therapy. Geets et al. described re-imaging with FDG PET scan during radiation treatment in head-and-neck patients ${ }^{47}$. After an average dose of $46 \mathrm{~Gy}, 18$ patients received a repeat PET-scan, which was hard to interpret due to inflammation. In our patients with 
NSCLC we did not encounter this problem. De Geus-Oei et al. showed in 22 patients with head-and-neck tumours a median decrease of relative change of SUV max of $4 \%$, comparing the PET scan after hyperoxygenation to the baseline $\operatorname{scan}^{27}$. Lin et al. described repeated PET-scans during treatment in cervical patients, but no results concerning the (maximal) SUV were investigated in this study ${ }^{8}$. In both the study of Lin and Geets, most patients received concurrent chemo-radiation, while in the study of de Geus-Oei et al. patients were treated with ARCON.

By using the EORTC criteria to evaluate the metabolic response after 70 days to compare the time trends of the SUV $V_{\max }$ of responders and non-responders, we could confirm the findings of Mac Manus et al. that patients showing a metabolic response after radiotherapy had a better overall survival than the non-responders $(p=0.005)^{25}$. A significant difference in the time trends of $S U V_{\text {max }}$ during radiotherapy between metabolic responders and non-responders was found. Metabolic non-responders had a higher SUV $\mathrm{Vax}_{\max }$ at all time points investigated and showed a significant increase in SUV $_{\max }$ during the first week of irradiation followed by a decrease. In contrast, the responders showed a stable SUV $_{\max }$ during irradiation. The number of included patients was however too small to calculate the predictive value of the changes in $\mathrm{SUV}_{\max }$ during a course of radiation.

The question thus remains what causes the observed time trends and their relation with subsequent response. A first hypothesis is that the increase of $S U V_{\max }$ during the first week is due to inflammation, since a rather high median dose of $19.8 \pm 4.1 \mathrm{~Gy}$ (1.8 Gy BID) was already delivered at the time point of the first repeat PET-CT scan. Radiation is known to be a potent inducer of inflammation in several tissues ${ }^{49-51}$. However, this cannot explain the differences in metabolic responders and nonresponders, since one would also expect inflammatory changes in the group of responders. Another possibility is that the $S_{U} V_{\max }$ provides information related to intrinsic biological characteristics of the tumour, such as tumour hypoxia. As hypoxia is strongly related to therapy resistance and the occurrence of metastases, this hypothesis is particularly attractive. The prognostic importance of the FDG uptake before therapy further underscores this hypothesis ${ }^{17-21}$. Hypoxia might play a role in the uptake of FDG, since it leads via the Hypoxia Inducible Factor-1a (HIF-1a) pathway to up regulation of glucose transporters. However, contradictory findings concerning the relation between FDG uptake and hypoxia have been published ${ }^{52-59}$.

Although the radiation dose (range between 46.8 Gy and $79.2 \mathrm{~Gy}$ in this study) could have influenced the response rate, a dose-response relation could not be established in this series, presumably due to the limited number of patients ${ }^{4,6}$. Another possibility is that the response on induction chemotherapy may also affect the subsequent response to radiation. However, this could not be shown in our study. To fully exclude the influence of induction chemotherapy it would have been best to perform the first PET-CT scan before start of any treatment. Although most of our patients underwent a PET(-CT) scan before starts of chemotherapy, most of these scans were performed at different PET(-CT) scanners in affiliated hospitals. Since maximal SUV of different scanners cannot be compared directly, we were not able to encompass maximal SUV before start of chemotherapy into our results. 
Lastly, the overall treatment time of radiotherapy might influence response after radiotherapy ${ }^{60}$. We did not find such a correlation between OTT of radiotherapy and the metabolic response rate, but this may be due to a short OTT (less than 5 weeks) in all patients. However, like for radiation dose, a difference in OTT could not explain why a change in FDG uptake on day 7 could affect response after 70 days. Our present working hypothesis is that the difference in time trends of $S_{U} V_{\max }$ as observed between responders and non-responders might reflect a complex relationship between several factors, like changes in blood flow as well as changes in the extracellular compartment, together with several intrinsic tumour properties, which all contribute to the uptake of the FDG tracer. Further in-depth studies, including analysis of several plasma markers at different time points, to address this question are ongoing.

In conclusion, a large heterogeneity in changes in $\mathrm{SUV}_{\max }$ was observed during thoracic radiation. Differences in time trends of SUV $V_{\max }$ during radiotherapy were found between metabolic responders and non-responders. Further research addressing the question whether these time trends can predict response, the biological meaning of the different time trends and whether it finally might enable individualized adjustments of treatment is ongoing. The final goal being prediction of response early during the course of radiotherapy to enable early adjustments, like prescribing a higher total dose, adjusting the fractionation scheme or adding another form of treatment. 


\section{References}

1. Sause W, Kolesar P, Taylor SI, et al. Final results of phase III trial in regionally advanced unresectable non-small cell lung cancer: Radiation Therapy Oncology Group, Eastern Cooperative Oncology Group, and Southwest Oncology Group. Chest 2000;117:358-364.

2. Saunders $M$, Dische $S$, Barrett $A$, et al. Continuous, hyperfractionated, accelerated radiotherapy (CHART) versus conventional radiotherapy in non-small cell lung cancer: mature data from the randomised multicentre trial. CHART Steering committee. Radiother Oncol 1999;52:137-148.

3. Novello S, le Chevalier T. Is there a standard strategy in the management of locally advanced non-small cell lung cancer? Lung Cancer 2001;34 Suppl 4:S9-14.

4. Martel MK, Ten Haken RK, Hazuka MB, et al. Estimation of tumor control probability model parameters from 3-D dose distributions of non-small cell lung cancer patients. Lung Cancer $1999 ; 24: 31-37$.

5. Belderbos J, Heemsbergen W, Hoogeman M, et al. Acute esophageal toxicity in non-small cell lung cancer patients after high dose conformal radiotherapy. Radioth Oncol 2005;75:157-164.

6. Cox JD, Azarnia N, Byhardt RW, et al. A randomized phase I/II trial of hyperfractionated radiation therapy with total doses of $60.0 \mathrm{~Gy}$ to $79.2 \mathrm{~Gy}$ : possible survival benefit with greater than or equal to $69.6 \mathrm{~Gy}$ in favorable patients with Radiation Therapy Oncology Group stage III non-small-cell lung carcinoma: report of Radiation Therapy Oncology Group 83-11. J Clin Oncol 1990;8:1543-1555.

7. Furuse K, Fukuoka M, Kawahara M, et al. Phase III study of concurrent versus sequential thoracic radiotherapy in combination with mitomycin, vindesine, and cisplatin in unresectable stage III non-small-cell lung cancer. J Clin Oncol 1999;17:2692-2699.

8. Kong FM, Ten Haken RK, Schipper MJ, et al. High-dose radiation improved local tumor control and overall survival in patients with inoperable/unresectable non-small-cell lung cancer: longterm results of a radiation dose escalation study. Int J Radiat Oncol Biol Phys 2005;63:324333.

9. Onishi H, Kuriyama K, Yamaguchi M, et al. Concurrent two-dimensional radiotherapy and weekly docetaxel in the treatment of stage III non-small cell lung cancer: a good local response but no good survival due to radiation pneumonitis. Lung Cancer 2003;40:79-84.

10. Kong FM, Ten Haken R, Eisbruch A, et al. Non-small cell lung cancer therapy-related pulmonary toxicity: an update on radiation pneumonitis and fibrosis. Semin Oncol 2005;32:S42-54.

11. Rodrigues G, Lock M, D'Souza D, et al. Prediction of radiation pneumonitis by dose - volume histogram parameters in lung cancer--a systematic review. Radiother Oncol 2004;71:127-138.

12. Seppenwoolde Y, Lebesque JV, de Jaeger K, et al. Comparing different NTCP models that predict the incidence of radiation pneumonitis. Normal tissue complication probability. Int J Radiat Oncol Biol Phys 2003;55:724-735.

13. Grigsby PW, Siegel BA, Dehdashti F, et al. Posttherapy (18F) fluorodeoxyglucose positron emission tomography in carcinoma of the cervix: response and outcome. $J$ Clin Oncol $2004 ; 22: 2167-2171$.

14. Forshaw MJ, Gossage JA, Mason RC. Neoadjuvant chemotherapy for oesophageal cancer: the need for accurate response prediction and evaluation. Surgeon 2005;3:373-382, 422.

15. Xue F, Lin LL, Dehdashti F, et al. F-18 fluorodeoxyglucose uptake in primary cervical cancer as an indicator of prognosis after radiation therapy. Gynecol Oncol 2006;101:147-151.

16. Eschmann SM, Friedel G, Paulsen F, et al. Is standardised (18)F-FDG uptake value an outcome predictor in patients with stage III non-small cell lung cancer? Eur $\mathrm{J} \mathrm{Nucl} \mathrm{Med} \mathrm{Mol} \mathrm{Imaging}$ 2005.

17. Ahuja V, Coleman RE, Herndon J, et al. The prognostic significance of fluorodeoxyglucose positron emission tomography imaging for patients with nonsmall cell lung carcinoma. Cancer 1998;83:918-924.

18. Borst GR, Belderbos JS, Boellaard R, et al. Standardised FDG uptake: a prognostic factor for inoperable non-small cell lung cancer. Eur J Cancer 2005;41:1533-1541.

19. Downey RJ, Akhurst T, Gonen M, et al. Preoperative F-18 fluorodeoxyglucose-positron emission tomography maximal standardized uptake value predicts survival after lung cancer resection. J Clin Oncol 2004;22:3255-3260. 
20. Higashi K, Ueda Y, Arisaka Y, et al. 18F-FDG uptake as a biologic prognostic factor for recurrence in patients with surgically resected non-small cell lung cancer. J Nucl Med 2002;43:3945.

21. Vansteenkiste JF, Stroobants SG, Dupont PJ, et al. Prognostic importance of the standardized uptake value on (18)F-fluoro-2-deoxy-glucose-positron emission tomography scan in nonsmall-cell lung cancer: An analysis of 125 cases. Leuven Lung Cancer Group. J Clin Oncol 1999;17:3201-3206.

22. Mac Manus MP, Hicks RJ, Matthews JP, et al. Positron emission tomography is superior to computed tomography scanning for response-assessment after radical radiotherapy or chemoradiotherapy in patients with non-small-cell lung cancer. J Clin Oncol 2003;21:1285-1292.

23. Weber WA, Petersen V, Schmidt B, et al. Positron emission tomography in non-small-cell lung cancer: prediction of response to chemotherapy by quantitative assessment of glucose use. $J$ Clin Oncol 2003;21:2651-2657.

24. Cerfolio RJ, Bryant AS, Winokur TS, et al. Repeat FDG-PET after neoadjuvant therapy is a predictor of pathologic response in patients with non-small cell lung cancer. Ann Thorac Surg 2004;78:1903-1909; discussion 1909.

25. Mac Manus MP, Hicks RJ, Matthews JP, et al. Metabolic (FDG-PET) response after radical radiotherapy/chemoradiotherapy for non-small cell lung cancer correlates with patterns of failure. Lung Cancer 2005;49:95-108.

26. Hoekstra CJ, Stroobants SG, Smit EF, et al. Prognostic relevance of response evaluation using (18F)-2-fluoro-2-deoxy-D-glucose positron emission tomography in patients with locally advanced non-small-cell lung cancer. J Clin Oncol 2005;23:8362-8370.

27. de Geus-Oei LF, Kaanders JH, Pop LA, et al. Effects of hyperoxygenation on FDG-uptake in head-and-neck cancer. Radiother Oncol 2006;2006 Jul 1; (Epub ahead of print).

28. Erdi YE, Macapinlac $\mathrm{H}$, Rosenzweig KE, et al. Use of PET to monitor the response of lung cancer to radiation treatment. Eur J Nucl Med 2000;27:861-866.

29. Dose Schwarz J, Bader M, Jenicke L, et al. Early prediction of response to chemotherapy in metastatic breast cancer using sequential 18F-FDG PET. J Nucl Med 2005;46:1144-1150.

30. Gallamini A, Rigacci L, Merli F, et al. The predictive value of positron emission tomography scanning performed after two courses of standard therapy on treatment outcome in advanced stage Hodgkin's disease. Haematologica 2006;91:475-481.

31. Brahme A. Biologically optimized 3-dimensional in vivo predictive assay-based radiation therapy using positron emission tomography-computerized tomography imaging. Acta Oncol 2003;42:123-136.

32. Therasse P, Arbuck SG, Eisenhauer EA, et al. New guidelines to evaluate the response to treatment in solid tumors. European Organization for Research and Treatment of Cancer, National Cancer Institute of the United States, National Cancer Institute of Canada. J Natl Cancer Inst 2000;92:205-216.

33. Thie JA. Understanding the standardized uptake value, its methods, and implications for usage. J Nucl Med 2004;45:1431-1434.

34. ICRU. Prescribing, Recording and Reporting Photon Beam Therapy ICRU Report 50. 1993; ISBN 0-913394-48-3.

35. Miften $M$, Wiesmeyer $M$, Monthofer $S$, et al. Implementation of FFT convolution and multigrid superposition models in the FOCUS RTP system. Phys Med Biol 2000;45:817-833.

36. Young $\mathrm{H}$, Baum R, Cremerius $\mathrm{U}$, et al. Measurement of clinical and subclinical tumour response using (18F)-fluorodeoxyglucose and positron emission tomography: review and 1999 EORTC recommendations. European Organization for Research and Treatment of Cancer (EORTC) PET Study Group. Eur J Cancer 1999;35:1773-1782.

37. De Ruysscher D, Wanders S, Minken A, et al. Effects of radiotherapy planning with a dedicated combined PET-CT-simulator of patients with non-small cell lung cancer on dose limiting normal tissues and radiation dose-escalation: a planning study. Radiother Oncol 2005;77:5-10.

38. van Der Wel A, Nijsten S, Hochstenbag $M$, et al. Increased therapeutic ratio by 18FDG-PET CT planning in patients with clinical CT stage N2-N3M0 non-small-cell lung cancer: a modeling study. Int J Radiat Oncol Biol Phys 2005;61:649-655. 
39. Ashamalla $\mathrm{H}$, Rafla S, Parikh $\mathrm{K}$, et al. The contribution of integrated PET/CT to the evolving definition of treatment volumes in radiation treatment planning in lung cancer. Int J Radiat Oncol Biol Phys 2005;63:1016-1023.

40. Bradley J, Thorstad WL, Mutic S, et al. Impact of FDG-PET on radiation therapy volume delineation in non-small-cell lung cancer. Int J Radiat Oncol Biol Phys 2004;59:78-86.

41. Erdi YE, Rosenzweig K, Erdi AK, et al. Radiotherapy treatment planning for patients with nonsmall cell lung cancer using positron emission tomography (PET). Radiother Oncol 2002;62:5160.

42. Vanuytsel L, Vansteenkiste JF, Stroobants SG, et al. The impact of (18)F-fluoro-2-deoxy-Dglucose positron emission tomography (FDG-PET) lymph node staging on the radiation treatment volumes in patients with non-small cell lung cancer. Radiother Oncol 2000;55:317-324.

43. Pottgen C, Levegrun S, Theegarten D, et al. Value of 18F-fluoro-2-deoxy-D-glucose-positron emission tomography/computed tomography in non-small-cell lung cancer for prediction of pathologic response and times to relapse after neoadjuvant chemoradiotherapy. Clin Cancer Res 2006;12:97-106.

44. Gagel B, Reinartz P, Demirel C, et al. (18F) fluoromisonidazole and (18F) fluorodeoxyglucose positron emission tomography in response evaluation after chemo-/radiotherapy of non-smallcell lung cancer: a feasibility study. BMC Cancer 2006;6:51.

45. van Baardwijk A, Baumert BG, Bosmans G, et al. The current status of FDG-PET in tumour volume definition in radiotherapy treatment planning. Cancer Treat Rev 2006;32:245-260.

46. Rahmouni A, Luciani A, Itti E. MRI and PET in monitoring response in lymphoma. Cancer Imaging 2005;5 Spec No A:S106-112.

47. Geets X, Daisne JF, Tomsej M, et al. Impact of the type of imaging modality on target volumes delineation and dose distribution in pharyngo-laryngeal squamous cell carcinoma: comparison between pre- and per-treatment studies. Radiother Oncol 2006.

48. Lin LL, Yang Z, Mutic S, et al. FDG-PET imaging for the assessment of physiologic volume response during radiotherapy in cervix cancer. Int J Radiat Oncol Biol Phys 2006.

49. Johnston CJ, Williams JP, Elder A, et al. Inflammatory cell recruitment following thoracic irradiation. Exp Lung Res 2004;30:369-382.

50. Rube $C E$, Rodemann HP, Rube $C$. (The relevance of cytokines in the radiation-induced lung reaction. Experimental basis and clinical significance). Strahlenther Onkol 2004;180:541-549.

51. Van der Meeren A, Monti P, Lebaron-Jacobs L, et al. Characterization of the acute inflammatory response after irradiation in mice and its regulation by interleukin 4 (Il4). Radiat Res 2001;155:858-865.

52. Burgman $\mathrm{P}$, Odonoghue $\mathrm{JA}$, Humm JL, et al. Hypoxia-Induced increase in FDG uptake in MCF7 cells. J Nucl Med 2001;42:170-175.

53. Clavo AC, Brown RS, Wahl RL. Fluorodeoxyglucose uptake in human cancer cell lines is increased by hypoxia. J Nucl Med 1995;36:1625-1632.

54. Minn $H$, Clavo AC, Wahl RL. Influence of hypoxia on tracer accumulation in squamous-cell carcinoma: in vitro evaluation for PET imaging. Nucl Med Biol 1996;23:941-946.

55. Pugachev A, Ruan S, Carlin S, et al. Dependence of FDG uptake on tumor microenvironment. Int J Radiat Oncol Biol Phys 2005;62:545-553.

56. Tanaka T, Furukawa T, Fujieda S, et al. Double-tracer autoradiography with Cu-ATSM/FDG and immunohistochemical interpretation in four different mouse implanted tumor models. NuCl Med Biol 2006;33:743-750.

57. Vaupel P. The role of hypoxia-induced factors in tumor progression. Oncologist 2004;9 Suppl 5:10-17.

58. Zhang JZ, Behrooz A, Ismail-Beigi F. Regulation of glucose transport by hypoxia. Am J Kidney Dis $1999 ; 34: 189-202$.

59. Zimny M, Gagel B, Dimartino E, et al. FDG-a marker of tumour hypoxia? A comparison with ((18)F)fluoromisonidazole and po (2)-polarography in metastatic head and neck cancer. Eur J Nucl Med Mol Imaging 2006.

60. Bentzen SM, Thames HD. Clinical evidence for tumor clonogen regeneration: interpretations of the data. Radiother Oncol 1991;22:161-166. 

The clinical implications:
the role of individualized
radiotherapy in NSCLC

The clinical implications:
the role of individualized
radiotherapy in NSCLC

C

\begin{tabular}{l} 
The clinical implications: \\
the role of individualized \\
radiotionerapy in NSCLC \\
\hline
\end{tabular}

\begin{tabular}{l} 
The clinical implications: \\
the role of individualized \\
radiotionerapy in NSCLC \\
\hline
\end{tabular}

\begin{tabular}{l} 
The clinical implications: \\
the role of individualized \\
radiotionerapy in NSCLC \\
\hline
\end{tabular}

\begin{tabular}{l} 
The clinical implications: \\
the role of individualized \\
radiotionerapy in NSCLC \\
\hline
\end{tabular}

d

The clinical implications:
the role of individualized
radiotherapy in NSCLC

The clinical implications:
the role of individualized
radiotherapy in NSCLC

$x^{2}$

(1)
. 



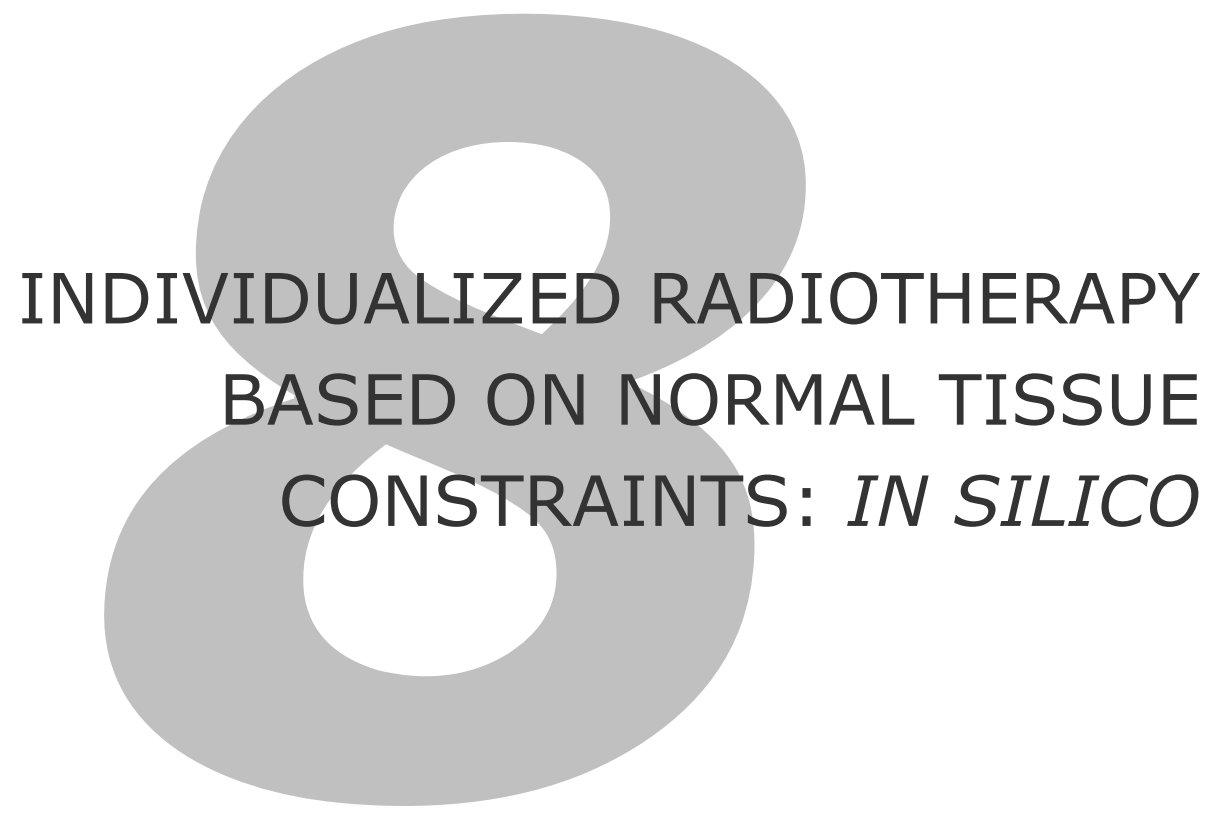

A. van Baardwijk, G. Bosmans, S.M. Bentzen, L. Boersma, A. Dekker, R. Wanders, B.G. Wouters, P. Lambin and D. De Ruysscher. Radiation dose prescription for nonsmall cell lung cancer based on normal tissue dose constraints: an in silico clinical trial.

Int J Rad Oncol Biol 2008 (in press). 


\section{Abstract}

Purpose: Local tumor recurrence remains a major problem in patients with inoperable non-small cell lung cancer (NSCLC) receiving radiotherapy. We investigated the theoretical gain in estimated tumor control probability (TCP) from using an individualized maximal tolerable dose (MTD) prescription, both for conventional and accelerated fractionation schemes.

Materials and Methods: For 64 NSCLC patients, 5 treatment plans were compared dependent upon normal tissue dose constraints for lung and spinal cord. The first two employed a classic fractionation ( $2 \mathrm{~Gy} /$ day, 5 days/week) to a total dose of $60 \mathrm{~Gy}$ $\left(\mathrm{QD}_{\text {classic }}\right)$ or determined by individualized MTD ( $\left.Q D_{\text {MTD }}\right)$. The third scheme assumed a hypofractionated schedule of 2.75 Gy fractions $\left(Q D_{\text {hypofr }}\right)$, whilst the fourth and fifth were based on hyperfractionation and acceleration, (1.8 Gy twice daily, either BID classic

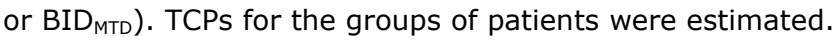

Results: The mean biological equivalent dose in 2 Gy fractions for tumor, corrected for

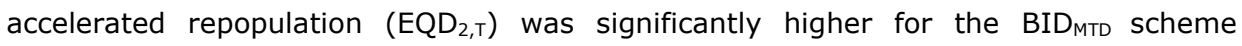
$(62.1 \mathrm{~Gy})$ than for any other scheme (QD classic $47.5 \mathrm{~Gy}$, QD $D_{\text {MTD }} 52.0 \mathrm{~Gy}$, QD hypofr $56.9 \mathrm{~Gy}$ and BID classic $56.9 \mathrm{~Gy}, \mathrm{p}<0.001$ ). While both dose-escalation (QD $\mathrm{QTT}_{\mathrm{D}}$ ) and hypofractionation ( $Q D_{\text {hypofr }}$ ) resulted in an increase in mean estimated TCP of respectively $5.6 \%(p<0.001)$ and $14.6 \%(p<0.001)$ compared with $Q_{\text {classic, the combination of }}$ escalation and acceleration (BID ${ }_{\text {MTD }}$ ) improved the mean estimated TCP with $26.4 \%$ $(p<0.001)$.

Conclusions: This planning study shows a large gain in estimated TCP using an MTD scheme with $1.8 \mathrm{~Gy}$ fractions BID compared with other fractionation schedules. Clinical studies implementing this concept are ongoing. 


\section{Introduction}

Although the prognosis of patients with inoperable lung cancer has improved, local tumor control is still not achieved in a large proportion of patients ${ }^{1-4}$. Approaches to improve loco-regional control and survival include the addition of chemotherapy and increasing the radiation dose $\mathrm{e}^{1,4-9}$. Several authors have reported dose-escalation studies in non-small cell lung cancer (NSCLC) $)^{5,7,8,10-12}$. Kong et al. analyzed 109 patients with stage I-III NSCLC and showed a better overall survival and local control rate in the highest dose group ${ }^{7}$. Mathematical modeling suggested that a high dose, probably above 84 Gy in daily 2 Gy fractions, is needed to achieve $50 \%$ or more tumor control probability $(\mathrm{TCP})^{13}$.

However, in addition to the total tumor dose (TTD), the overall treatment time (OTT) in which the radiation treatment is given is an important determinant of outcome as demonstrated in the continous, hyperfractionated, accelarted radiotherapy (CHART) trial $^{3}$. This study showed an absolute improvement in 2-year survival and an increase in local tumor control comparing 54 Gy in 12 consecutive days (1.5 Gy three times daily) with a conventional scheme of 30 2-Gy fractions, once daily in 6 weeks. In the continous hyperfractionated accelarated radiotherapy-weekend less (CHARTWEL) trial $60 \mathrm{~Gy}$ in 2.5 weeks (three daily fractions of $1.5 \mathrm{~Gy}$ ) was compared with $66 \mathrm{~Gy}$ in 6.5 weeks ( 2 Gy once daily) ${ }^{14}$. In this study both arms showed an equal overall survival, supporting that a similar survival could be obtained with a lower TTD when the OTT is shortened.

Concurrent chemo-radiation leads to an increase in overall survival compared with sequential therapy, but at the cost of a much greater incidence of acute toxicity, mainly esophagitis ${ }^{9,5-19}$. This gain in survival has mainly been attributed to an improvement in local tumor control ${ }^{17,18}$. Nevertheless, local relapse remains the initial site of failure in about one-third of the patients ${ }^{16-18}$.

It has been estimated that the best local control rate could be achieved by delivering the highest possible dose in an OTT of $<5$ weeks $^{20}$. However, dose-escalation is limited by damage to normal tissues. Dosimetric factors, such as the relative volume receiving a certain dose (e.g. the $V_{5}-V_{65}$ ) and the mean lung dose (MLD) appear to be useful for estimating the probability to develop radiation pneumonitis ${ }^{21,22}$. Several authors performed phase I/II studies to evaluate the feasibility of escalating dose based on bins using different modes to assess lung injury in different risk groups $s^{5,11,23-25}$.

We hypothesized that the best achievable radiation schedule would include the highest TTD based on individualized normal tissue dose constraints, delivered in an OTT of $<5$ weeks. Hypofractionated schedules, as well as hyperfractionated schedules combined with acceleration ( 2 fractions daily) are methods to deliver a high TTD within a short OTT. A hypofractionated schedule is more convenient for the patient. However, a schedule with two fractions daily might lead to a relative sparing of normal tissues or could allow high dose irradiation of tumors next to critical organs.

The aim of the current study was to investigate the theoretical gain in TCP using the concept of an individualized dose prescription, based on normal tissue dose constraints. 
Several fractionation schedules, including classic fractionation, hypofractionation and hyperfractionation combined with accelerated treatment are compared in NSCLC patients.

\section{Materials and Methods}

\section{Patients}

Radiotherapy plans from 64 consecutive patients with medically inoperable or locally advanced NSCLC stage I-IIIB, enrolled in different trials at MAASTRO clinic, were retrieved from archive for this planning study ${ }^{26-28}$. The clinical stage distribution was: stage IA in 2 cases, 6 patients with IB, 1 patient with IIB, 24 patients with IIIA and 31 patients with IIIB. The treatment plans were calculated using an individualized prescribed maximal tolerable dose (MTD) based on normal tissue dose constraints for lung and spinal cord. Because most patients had great vessels and/or the main bronchi included in the target volume, we applied a maximal allowed TTD. Based on the study of Marks et al. the maximal allowed TTD were ${ }^{12}: 76.0 \mathrm{~Gy}$ in $2.0 \mathrm{~Gy}$ fractions once daily, $66.0 \mathrm{~Gy}$ in $2.75 \mathrm{~Gy}$ fractions once daily and $79.2 \mathrm{~Gy}$ in $1.8 \mathrm{~Gy}$ fractions twice daily.

\section{Radiotherapy planning}

To investigate both the role of dose-escalation as well as decreasing OTT by twice daily irradiation or hypofractionation, we calculated five different treatment plans for each patient. A three-dimensional conformal treatment planning was performed, that was individually optimized to reach the highest possible TTD within the normal tissue constraints. For a given patient, all five plans used the same beam arrangement. All plans were in accordance with the International commission on Radiation Units and Measurements guidelines ${ }^{29}$ :

1. The first plan ( $\mathrm{QD}_{\text {classic }}$ ) was a classic dose prescription of $60 \mathrm{~Gy}$, once daily $2 \mathrm{~Gy}$ fractions (QD) or a lower TTD if the normal tissue dose constraints were reached at a lower dose.

2. The second plan ( $Q D_{\text {MTD }}$ ) was calculated based on an individualized, maximal tolerable dose depending on normal tissue dose constraints with a maximum TTD of $76 \mathrm{~Gy}$ in $2.0 \mathrm{~Gy}$ fractions once daily.

3. The third plan (QDhypofr) was based on a hypofractionated schedule of $2.75 \mathrm{~Gy}$ fractions once daily till a maximal TTD of $66 \mathrm{~Gy}^{30,31}$ applying the same dose constraints.

4. The fourth plan (BIDclassic) was calculated to investigate the contribution of acceleration, based on an TTD of $61.2 \mathrm{~Gy}(\mathrm{EQD} 2, \mathrm{~T}=60.2 \mathrm{~Gy}$ ) in $1.8 \mathrm{~Gy}$ fractions twice daily.

5. The fifth plan (BIDMTD) combined the effect of dose escalation and acceleration. The same constraints as in QDMTD were applied. The TTD was prescribed in 1.8 Gy fractions, BID, 5 days a week, to a maximal TTD of 79.2 Gy.

For all plans we used a normalized mean lung dose (nMLD) of maximal $19.0 \pm 1.0 \mathrm{~Gy}$ as the constraint. Based on published data, this constraint was estimated to yield a probability of $\geq$ grade 2 pneumonitis of about $15 \%$ (maximum $20 \%)^{21}$. For the spinal 
cord a maximal dose of $54 \pm 0.5$ Gy (in biological equivalent dose in 2 Gy fractions, $\mathrm{EQD}_{2, \mathrm{M}}$ ) was accepted ${ }^{32}$. No specific dose constraint for the esophagus was used as acute esophagitis was not considered to be dose-limiting when radiation alone was delivered.

Contouring of the lungs was done automatically by the treatment planning system (XiO, CMS, St. Louis, MO). The spinal cord was considered to be at the inner margin of the bony spinal canal. Radiotherapy planning was performed using inhomogeneity corrections based on a convolution algorithm. Both normal tissue contours as well as target volumes were outlined on each slice. The gross tumor volume (GTV) was the primary tumor on CT and lymph nodes positive on PET scan or proven to be positive on mediastinoscopy or biopsy. For the delineation of the primary tumor on CT the windowlevel (W-L) setting for lung (W 1700; L -300) was used, whereas for the anatomical delineation of PET positive nodes the mediastinal W-L setting (W 600; L 40). No elective nodal irradiation was carried out ${ }^{5,33}$. The clinical target volume (CTV) was defined as the GTV plus a $5 \mathrm{~mm}$ margin. For the planning target volume (PTV) another $10 \mathrm{~mm}$ margin was added for the primary tumor and a $5 \mathrm{~mm}$ for the lymph nodal areas.

The linear quadratic model was used to calculate the biological equivalent dose for tumor in $2 \mathrm{~Gy}$ fractions $\left(\mathrm{EQD}_{2, \mathrm{~T}}\right)$, corrected for the overall treatment time:

$$
E Q D_{2, T}=D \cdot \frac{d+(\alpha / \beta)}{2+(\alpha / \beta)}-\left(T_{r e f}-T\right) \cdot D_{\text {prolif }}
$$

Where $\mathrm{D}$ is the total radiation dose, $\mathrm{d}$ the dose per fraction, $\mathrm{T}$ the overall OTT. An $a / \beta$ ratio of $10 \mathrm{~Gy}$ was used for tumor ${ }^{34}$. As we assumed a 3-week lag period before start of accelerated repopulation, $T_{\text {ref }}$ was chosen at 21 days. Before day $21\left(T<T_{\text {ref }}\right)$ repopulation was assumed to be zero $\left(D_{\text {prolif }}=0\right)$; after day $21\left(T>T_{\text {ref }}\right)$ the dose recovered per day in 2 Gy equivalent fractions ( $D_{\text {prolif }}$ ) was assumed to be $0.6 \mathrm{~Gy}^{35}$.

To assess the maximal dose for the spinal cord $\left(E Q D_{2, M}\right)$ for the schemes with once daily fractions ( $Q D_{\text {classic }}, \mathrm{QD}_{\mathrm{MTD}}, \mathrm{QD}_{\text {hypofr }}$ ) the standard linear quadratic model was used ( $a / \beta$ ratio of $2 \mathrm{~Gy}$ ), assuming that sufficient time was allowed for complete repair between fractions. However, for the BID schemes (BID classic, $_{\text {BID }}$ MTD) the incomplete repair linear quadratic model was used ${ }^{34,36}$.

$$
E Q D_{2, M}=D \cdot \frac{d\left(1+H_{m}\right)+(\alpha / \beta)}{2+(\alpha / \beta)}
$$

where $D$ is the total radiation dose and $d$ the dose per fraction. An $a / \beta$ ratio of $2 \mathrm{~Gy}$ was used for spinal cord, and the amount of unrepaired damage was expressed by the factor $\mathrm{H}_{m}$. For the spinal cord a repair half-time of 3.8 hours was assumed ${ }^{37}$. Both incomplete repair between fractions (8-h interval), as well as incomplete repair overnight (16-h interval) was taken into account ${ }^{38}$.

For the normal lung tissue the mean lung dose, defined as the average dose throughout the lungs, was used as a dose constraint ${ }^{21}$. The MLD takes into account both the 
dose delivered as well as the volume irradiated, since the MLD transforms an inhomogeneous dose distribution into an equivalent uniform irradiation of the whole lung. However, the normalized mean (biological) lung dose (nMLD) could not be calculated directly for all patients, but was estimated from the mean physical lung dose similar to the method reported by Kwa et al. ${ }^{21}$ The following relation between the nMLD and the mean physical dose was obtained from a fit of data from 102 patients treated in our department.

$$
n M L D=0.90 * \text { mean physical lung dose }
$$

where nMLD is calculated with an $a / \beta$ ratio of $4 \mathrm{~Gy}$ for pneumonitis ${ }^{35}$. For the calculation of the mean physical lung dose, the volume of both lungs minus the GTV was considered ${ }^{9}$.

\section{Tumor Control Probability (TCP) calculations}

For TCP calculations, after a dose D, a logistic dose-response curve was assumed ${ }^{40}$ :

$$
\operatorname{TCP}(D)=\frac{1}{1+\left(\frac{D_{50}}{D}\right)^{4 \gamma}}
$$

where $D_{50}$ is the dose needed for a $50 \%$ TCP and $\gamma$ is the normalized slope of the sigmoid dose response curve near $D_{50}$. For calculations a $D_{50}$ of $70 \mathrm{~Gy}$, an $\gamma$ of 2.0 and for $D$ the $E Q D_{2, T}$ corrected for accelerated repopulation were used ${ }^{20}$. The TCP calculations did not take into account dose inhomogeneities within the PTV and/or incidental irradiation of nodal areas outside the GTV ${ }^{13}$. TCP calculations provided are thus merely an estimation of the TCP and are principally used for comparing the different schedules.

\section{Risk estimation for pneumonitis}

Following Kwa and colleagues ${ }^{21}$, the risk of pneumonitis ( $\geq$ grade 2 ) was estimated using the Lyman-Kutcher-Burman model $^{41-43}$ :

$$
\operatorname{NTCP}(D)=\frac{1}{\sqrt{2 \pi}} \int_{-\infty}^{t(D, V)} \exp \left(-\frac{1}{2} x^{2}\right) d x+c
$$

and

$$
t=\frac{n M L D-D_{50}}{m \cdot D_{50}}
$$

In this equation $D$ is the nMLD as mentioned above and $D_{50}$ is the nMLD which would result in a $50 \%$ risk to develop pneumonitis, being $31.8 \mathrm{~Gy}$ and $m=0.43$ and with an offset value (c) of 0 according to Kwa et al. ${ }^{21}$. 


\section{Statistics}

The SPSS software (version 15.0, SPSS Inc., Chicago, IL) was used for statistical analysis. Results are expressed as mean \pm standard deviation (SD) for TTD, EQD $2, T$, $\mathrm{EQD}_{2, \mathrm{M}}$ and $\mathrm{nMLD}$, whereas mean \pm standard error of mean (SEM) is applied to TCP estimations and risk estimation for pneumonitis. Since the data did not show a normal distribution, the Wilcoxon Signed Ranks Test was used to compare the results. P-values less than 0.05 were considered statistically significant.

\section{Ethics}

As this was a modeling study, patients were diagnosed and treated according to department guidelines. Patient data were anonymized. The study was approved by the Institutional Review Board.

\section{Results}

\section{Dose (TTD and EQD2 tumor)}

For both the individually escalated (MTD) schemes a significant higher mean TTD could be delivered: $\mathrm{QD}_{\text {MTD }} 66.6 \pm 9.2 \mathrm{~Gy}$ and BID $_{\text {MTD }} 65.3 \pm 11.3 \mathrm{~Gy}$ compared with classical and hypofractionated schedules: $\mathrm{QD}_{\text {classic }} 58.3 \pm 3.6 \mathrm{~Gy}$, QD hypofr $57.5 \pm 8.7 \mathrm{~Gy}$ and BID $_{\text {classic }} 58.4 \pm 5.1$ Gy (all $p<0.001$; Table 8.1 ). Out of the 14 patients $(21.9 \%$ ) not able to receive $60 \mathrm{~Gy}$ in the $\mathrm{QD}_{\text {classic }}$ scheme, 4 were limited by the $\mathrm{nMLD}$ at a TTD below 60 Gy. For $\mathrm{QD}_{\text {hypofr }}$ and $\mathrm{QD}_{\text {MTD }}$ the maximal dose could be reached in $24(37.5 \%)$ and 22 patients $(34.4 \%)$, respectively. The spinal cord dose was the limiting factor in half of the $\mathrm{QD}_{\text {MTD }}$ plans $(n=32)$ and in $40 \%(n=26)$ of the $\mathrm{QD}_{\text {hypo }}$ schedules.

Comparing the biological equivalent dose, corrected for accelerated repopulation, in 2 Gy fractions $\left(\mathrm{EQD}_{2, \mathrm{~T}}\right.$ ) for tumor we observed a mean $E \mathrm{ED}_{2, \mathrm{~T}}$ of $47.5 \pm 2.3$ Gy for $\mathrm{QD}_{\text {classic }}$ compared with $52.0 \pm 5.3 \mathrm{~Gy}$ for $\mathrm{QD}_{\text {MTD }}(\mathrm{p}<0.001)$. The mean $E Q D_{2, T}$ was higher for the hypofractionated schedule $(56.9 \pm 6.9 \mathrm{~Gy})$, than for the other once daily schemes $(p<0.001)$. The accelerated schemes showed a mean $\mathrm{EQD}_{2, \mathrm{~T}}$ of $56.9 \pm 3.8 \mathrm{~Gy}$ (BID ${ }_{\text {classic }}$ ) and $62.1 \pm 8.5$ Gy (BID $\left.{ }_{\text {MTD }}\right)$. The latter was significantly greater than for all other schemes $(p<0.001 ;$ Table 8.1$)$. 


\begin{tabular}{|c|c|c|c|c|c|}
\hline & $\mathrm{QD}_{\text {classic }}$ & $\mathrm{QD}_{\text {MTD }}$ & $\mathrm{QD}_{\text {hypo }}$ & BID $_{\text {classic }}$ & BID $_{\text {MTD }}$ \\
\hline & $60 \mathrm{~Gy} / 2.0 \mathrm{GyQD}$ & MTD/2.0 GyQD & MTD/2.75GyQD & $61.2 \mathrm{~Gy} / 1.8 \mathrm{GyBID}$ & MTD/1.8 GyBID \\
\hline \multicolumn{6}{|l|}{ TTD } \\
\hline Mean \pm SD & $58.3 \pm 3.6 \mathrm{~Gy}$ & $66.6 \pm 9.2 \mathrm{~Gy}$ & $57.5 \pm 8.7 \mathrm{~Gy}$ & $58.4 \pm 5.1 \mathrm{~Gy}$ & $65.3 \pm 11.3 \mathrm{~Gy}$ \\
\hline Range & (42.0-60.0 Gy) & $(42.0-76.0 \mathrm{~Gy})$ & $(33.0-66.0 \mathrm{~Gy})$ & (37.8-61.2 Gy) & (37.8-79.2 Gy) \\
\hline \multicolumn{6}{|c|}{ EQD2 tumor (corrected for repopulation) } \\
\hline Mean \pm SD & $47.5 \pm 2.3 \mathrm{~Gy}$ & $52.0 \pm 5.3 \mathrm{~Gy}$ & $56.9 \pm 6.9 \mathrm{~Gy}$ & $56.9 \pm 3.8 \mathrm{~Gy}$ & $62.1 \pm 8.5 \mathrm{~Gy}$ \\
\hline Range & (37.2-48.6 Gy) & (37.2-57.4 Gy) & (35.1-63.5 Gy) & (40.8-59.0 Gy) & (40.8-72.5 Gy) \\
\hline \multicolumn{6}{|c|}{ EQD2 spinal cord } \\
\hline Mean \pm SD & $42.1 \pm 10.8$ Gy & $47.1 \pm 9.9$ Gy & $47.2 \pm 9.7 \mathrm{~Gy}$ & $44.7 \pm 10.9 \mathrm{~Gy}$ & $48.8 \pm 9.5 \mathrm{~Gy}$ \\
\hline Range & (7.9-54.5 Gy) & (9.5-54.5 Gy) & (9.3-54.4 Gy) & (8.3-54.4 Gy) & (9.2-54.4 Gy) \\
\hline \multicolumn{6}{|l|}{ nMLD } \\
\hline Mean \pm SD & $12.0 \pm 4.5 \mathrm{~Gy}$ & $13.3 \pm 4.5 \mathrm{~Gy}$ & $11.5 \pm 4.3 \mathrm{~Gy}$ & $11.8 \pm 4.5 \mathrm{~Gy}$ & $13.0 \pm 4.5 \mathrm{~Gy}$ \\
\hline Range & (2.7-20.0 Gy) & (3.2-20.0 Gy) & (2.7-20.0 Gy) & (2.8-19.9 Gy) & (3.0-19.9 Gy) \\
\hline \multicolumn{6}{|c|}{ Estimated TCP } \\
\hline Mean \pm SE & $4.5 \% \pm 0.1 \%$ & $10.1 \% \pm 0.7 \%$ & $19.1 \% \pm 1.4 \%$ & $16.9 \% \pm 0.7 \%$ & $30.9 \% \pm 2.4 \%$ \\
\hline Range & $(0.6 \%-5.1 \%)$ & $(0.6 \%-17.0 \%)$ & $(0.4 \%-31.5 \%)$ & $(1.3 \%-20.3 \%)$ & $(1.3 \%-56.9 \%)$ \\
\hline \multicolumn{6}{|c|}{ 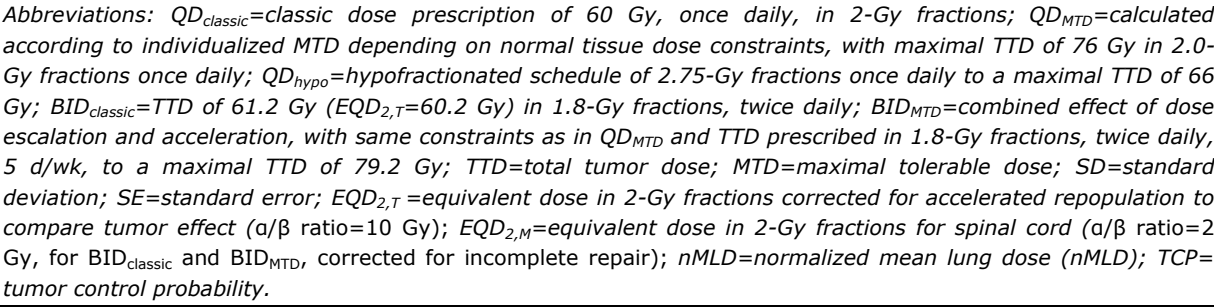 } \\
\hline
\end{tabular}

\section{Dose to normal tissues (EQD2 myelum and nMLD)}

Table 8.1 shows the $\mathrm{EQD}_{2, \mathrm{M}}$ and $\mathrm{nMLD}$ for all schemes. For the spinal cord, the mean $\mathrm{EQD}_{2, \mathrm{M}}$ BID classic $(44.7 \pm 0.9 \mathrm{~Gy})$ was significantly higher than the $\mathrm{EQD}_{2, \mathrm{M}} \mathrm{QD}_{\text {classic }}$ $(42.1 \pm 10.8 \mathrm{~Gy}, \mathrm{p}<0.001)$. No significant difference was observed for the nMLD comparing these schemes ( $\mathrm{BID}_{\text {classic }} 11.8 \pm 4.5 \mathrm{~Gy}$ and $\left.\mathrm{QD}_{\text {classic }} 12.0 \pm 4.5 \mathrm{~Gy}, \mathrm{p}=0.28\right)$. The nMLD was significant higher for the BID ${ }_{\text {MTD }}$ compared with $\mathrm{QD}_{\text {hypofr }}$ (mean nMLD $13.0 \pm 4.5 \mathrm{~Gy}$ and $11.5 \pm 4.3 \mathrm{~Gy}$, respectively, $\mathrm{p}<0.001$ ). The spinal cord dose was also somewhat higher for the BID $_{\text {MTD }}$ schedule (mean EQD 2 myelum $48.8 \pm 9.5$ Gy and 47.2 \pm 9.7 Gy respectively, $p<0.001$ ). Both MTD schemes showed higher $E Q D_{2, M}$ and nMLDs compared with the classic schemes (Table 8.1 and Figure 8.1).

\section{Tumor control probability (TCP)}

For the $\mathrm{QD}_{\text {MTD }}$ scheme an estimated TCP to a mean value of $10.1 \% \pm 0.7 \%$ was found (Table 8.1 and Figure 8.1). This is a limited, though significant increase compared with $\mathrm{QD}_{\text {classic }}$ (mean TCP of $4.5 \% \pm 0.1 \% ; \mathrm{p}<0.001$ ). Applying $\mathrm{QD}_{\text {hypofr }}$ and $\mathrm{BID}_{\text {classic, }}$ both could further improve the estimated TCP to a mean level of respectively $19.1 \% \pm 1.4 \%$ $(p<0.001)$ and $16.9 \% \pm 0.7 \%\left(p<0.001\right.$ compared with $Q_{\text {classic }}$ and $p=0.046$ compared with $\left.\mathrm{QD}_{\text {hypofr }}\right)$. The calculated TCP increased even further for the BID $\mathrm{D}_{\text {MTD }}$ scheme, showing an estimated mean TCP of $30.9 \% \pm 2.4 \% \quad(p<0.001$ compared with all other schemes). Comparing the estimated TCP of BID $\mathrm{BTD}_{\text {and }}$ and $\mathrm{QD}_{\text {classic, }}$ this resulted in a mean increase in TCP of $26.4 \% \pm 2.4 \%$. 


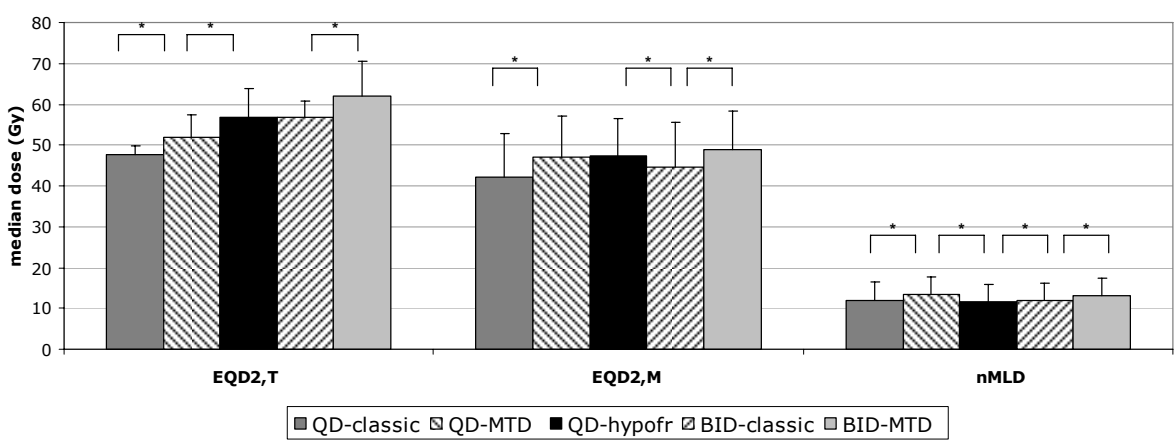

Figure 8.1 Calculations of biologic equivalent dose in 2-Gy fractions, corrected for accelerated proliferation $(E Q D 2, T)$ for tumor effect, spinal cord $(E Q D 2, M)$, and tumor control probability (TCP) (in percentages) estimations for all five schemes. Results expressed as mean \pm standard deviation for EQD2,T, $E Q D 2, M$, and $n M L D$ and mean \pm standard error of mean for estimated TCP. *Significant difference.

Although for the total group both dose escalation as well as acceleration showed a gain in the estimated TCP, this is not necessarily the case for each individual patient. In 15 (23.4\%) of 64 patients no dose-escalation could be performed due to the fact that the maximal tolerable dose was already reached at a TTD of 60 Gy or lower.

In $18(28.1 \%)$ of 64 patients, a lower TTD had to be prescribed for the BID MTD scheme compared with the $\mathrm{QD}_{\text {classic }}$ scheme, because of the spinal cord constraint. However, only in 1 patient $(1.6 \%)$ the BID $_{\text {MTD }}$ scheme would have resulted in a mainly unchanged estimated TCP compared with $\mathrm{QD}_{\text {classic }}$ (gain of $0.7 \%$ ). For the other 63 patients a gain in TCP was expected applying a combination of dose escalation and an accelerated fractionation schedule (Figure 8.2).

In the group of patients, where TTD was not limited by the spinal cord dose, a larger gain in estimated TCP could be achieved compared with those patients, in which the spinal cord dose was the limiting factor (gain in TCP $39.8 \% \pm 17.7 \%$ versus $15.3 \% \pm 11.1 \%$, respectively, $\mathrm{p}<0.001$ ). However, both the TCP as well as the change in TCP showed no significant dependence on the clinical stage of disease.

Figure 8.3 illustrates the improvement in therapeutic index as a result of doseescalation (Figure 8.3a; $\mathrm{QD}_{\text {MTD }}$ versus $\mathrm{QD}_{\text {classic }}$ ), as a result of acceleration (Figure 8.3b; $\mathrm{BID}_{\text {classic }}$ Vs. $\mathrm{QD}_{\text {classic }}$ ) and the combination of both (Figure 3c; BID $_{\text {MTD }}$ scheme compared with $\left.\mathrm{QD}_{\text {classic }}\right)$.

The gain in estimated TCP $(\triangle T C P)$ was plotted as function of the change in nMLD $(\triangle \mathrm{nMLD})$ comparing the different schemes. In 18 patients $(28.1 \%)$ the BID $_{\text {MTD }}$ scheme resulted in a lower nMLD compared with $\mathrm{QD}_{\text {classic }}$ (Figure 3c; dots left of line representing a delta nMLD of zero). Even these patients showed a gain in mean estimated TCP of $6.4 \% \pm 0.8 \%$. 


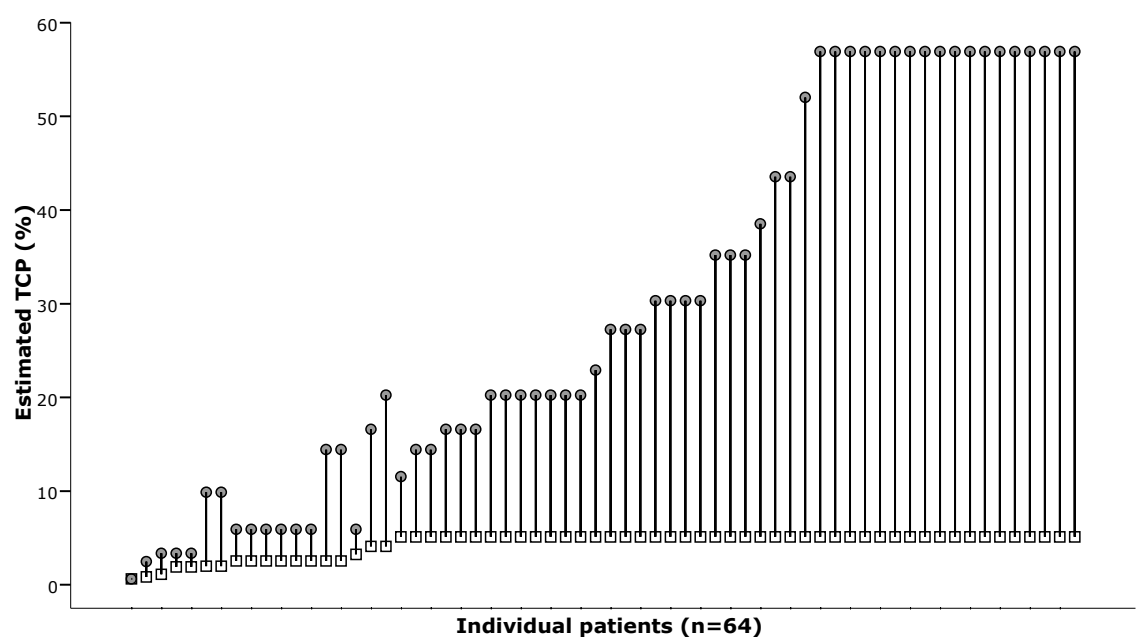

Figure 8.2 Gain in estimated tumor control probability (TCP) for all 64 individual patients comparing the classic scheme $\left(Q D_{\text {classic }} \square\right)$ and the maximal tolerable dose scheme in twice-daily fractions $\left(B I D_{M T D} \bullet\right)$.

\section{Estimated risk of pneumonitis}

The $\mathrm{QD}_{\text {classic }}$ scheme resulted in a mean estimated risk of pneumonitis of $8.3 \% \pm 0.6 \%$, whereas this was $9.9 \% \pm 0.6 \%$ for the $\mathrm{QD}_{\text {MTD }}(\mathrm{p}<0.001)$. For the accelerated schemes the mean estimated risk for pneumonitis was $8.3 \% \pm 0.6 \%$ and $9.5 \% \pm 0.6 \%$ for respectively BID $_{\text {classic }}$ and BID MTD $_{(}(p<0.001)$. No significant difference was observed comparing $\mathrm{BID}_{\text {classic }}$ and $\mathrm{QD}_{\text {hypofr }}(8.3 \% \pm 0.6 \%$ and $7.9 \% \pm 0.6 \%$, respectively, $\mathrm{p}=0.06)$. Moreover, the estimated risk for pneumonitis for the BID ${ }_{\text {MTD }}$ compared with QD $_{\text {classic }}$ was slightly higher for the total group of patient applying BID $_{\text {MTD }}(9.5 \% \pm 0.6 \%$ and $8.3 \% \pm 0.6 \%$ respectively, $\mathrm{p}<0.001)$.

\section{Discussion}

The purpose of this study was to estimate tumor control probabilities and the therapeutic index in a planning study comparing an individualized dose prescription based on lung and spinal cord constraints, with a classic non-escalated dose schedule. We showed that using an individualized dose prescription, a large gain in estimated TCP could be expected. With respect to the dose escalation in once daily fractions, the mean TTD in once daily 2.0 Gy fractions could be increased from $58.3 \mathrm{~Gy}$ ( $\mathrm{QD}_{\text {classic }}$ ) to $66.6 \mathrm{~Gy}$ for the QD $\mathrm{D}_{\text {MTD }}$ scheme resulting in a mean estimated gain in TCP of $5.6 \%$. Moreover, applying an accelerated scheme resulted in a greater benefit (mean gain in estimated TCP of $12.4 \%$, comparing BID $_{\text {classic }}$ with $\mathrm{QD}_{\text {classic }}$ ). The hypofractionation schedule resulted in a significant increase in mean estimated TCP of $14.6 \%$ compared with $\mathrm{QD}_{\text {classic. }}$ However, although the hypofractionation schedule is more convenient for the patient and the radiotherapy department, this scheme was still not able to catch up with the gain of the twice daily hyperfractionated, accelerated schedule (BID MTD $_{\text {versus }}$ $\mathrm{QD}_{\text {hypofr, }} 30.9 \%$ and $19.1 \%$ respectively, $\mathrm{p}<0.001$ ). These favorable results could be 

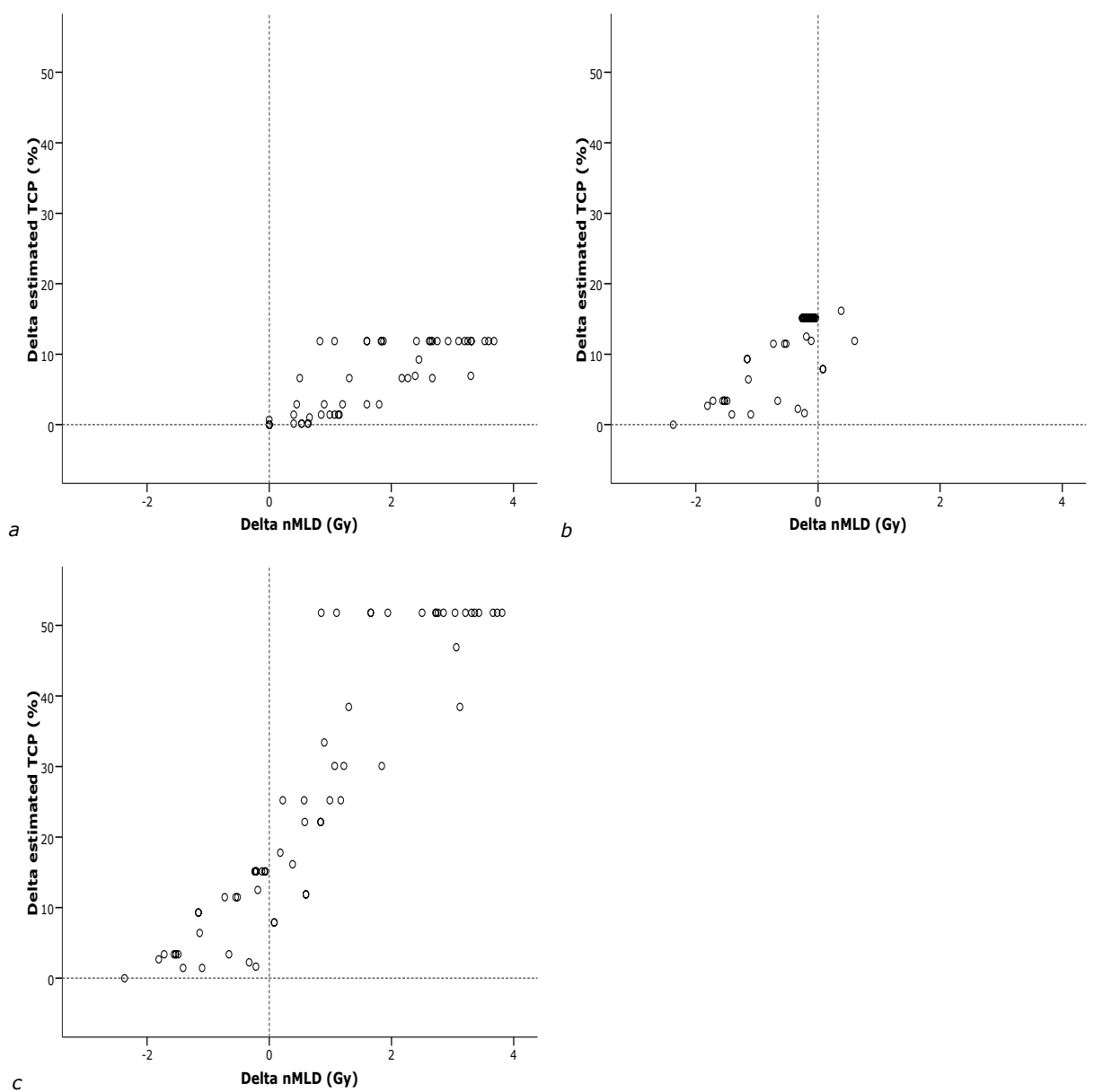

Fig. 8.3 Gain in estimated tumor control probability $(\triangle T C P)$ in relation to change in normalized mean lung dose $(\triangle n M L D)$ comparing two different schedules: (a) $\triangle T C P$ from dose escalation comparing classic scheme (QDclassic) and maximal tolerable dose scheme in once-daily fractions (QDMTD), (b) $\triangle T C P$ from acceleration comparing classic scheme (QDclassic) and hyperfractionated, accelerated classic scheme (BIDclassic), and (c) $\triangle T C P$ from combination of dose escalation and acceleration comparing classic scheme (QDclassic) and hyperfractionated, accelerated classic scheme to maximal tolerable dose (BIDMTD).

obtained since no effect of accelerated repopulation was expected to occur when the OTT was kept below 3 weeks $^{20}$. The TTD needed to achieve a 50\% tumor control probability is about 77 Gy for the accelerated scheme, which gives an $E Q D_{2, T}$ of $70 \mathrm{~Gy}^{13,20}$. For the classic schedule a much higher dose compared is needed to achieve the same control rate. In this study a radiation plan with an $\mathrm{EQD}_{2, \mathrm{~T}}$ of at least $70 \mathrm{~Gy}$ was feasible for 20 patients (31.3\%).

In this study we applied a maximal allowed TTD, since the majority of patients had stage III disease. Our maximal TTD for the BID MTD schedule of $79.2 \mathrm{~Gy}$ in $1.8 \mathrm{~Gy}$ fractions gives a comparable equivalent dose for tumor in $2 \mathrm{~Gy}$ fractions, corrected for the overall treatment time $\left(E Q D_{2, T}\right)$ with a scheme of $100 \mathrm{~Gy}$ in once daily fractions of 
2.0 Gy or a hypofractionated schedule of 92.25 Gy in once daily fractions of 2.25 Gy. Moreover, unlike some of the other dose-escalation studies ${ }^{5,24}$, we also included patients with involved supraclavicular nodes (N3 disease) and tumors located in the direct proximity of the spinal cord and great vessels, which inevitably leads to the inclusion of organs at risk into the planning target volume. Marks et al. defined in a group of stage III NSCLC the maximal tolerable dose at $80 \mathrm{~Gy}$, since above this dose complications like bronchial or tracheal stenosis, brocho-esophageal fistula and obstructions of the pulmonary artery are more frequently seen ${ }^{12}$. However, in a subset of patients, like patients with stage I or II NO disease, it might be safe to further escalate dose. One way to achieve this is by treating this subgroup of patients with extracranial radiosurgery.

Concerning late effects we observed a somewhat higher mean $\mathrm{EQD}_{2, \mathrm{M}}$ (late effects spinal cord, $a / \beta$ ratio of $2 \mathrm{~Gy}$ ) for the MTD schemes (47-49 Gy) compared with the classical schemes $(42-45 \mathrm{~Gy}, \mathrm{p}<0.001)$. A spinal cord dose of $54 \mathrm{~Gy}$, the dose constraint used in this study and in daily clinical practice at our department, probably leads to an incidence of radiation myelopathy of $5 \%$ or less ${ }^{32,44}$. With regard to lung injury, a higher mean nMLD was seen in the MTD schemes (13 Gy) compared with the classical schemes (12 Gy). This could result in a limited increase in the proportion of patients developing radiation induced lung injury. In this modeling study we used the model of Lyman to calculate the pneumonitis risk and applied a $D_{50}$ of $31.8 \mathrm{~Gy}$ and no offset value, as described by Kwa et al. for the total group of patients ${ }^{21,43}$. Applying the Lyman model we observed a limited increase in estimated risk for pneumonitis for the BID $_{\text {MTD }}$ schedule $(9.5 \%)$ compared with the $\mathrm{QD}_{\text {classic }}$ scheme $(8.3 \%, \mathrm{p}<0.001)$ and compared with the hypofractionated schedule $(7.9 \%, p<0.001)$. We realize that the risk for grade 2 or higher pneumonitis calculated for the accelerated schemes might be an underestimation, since like for tumors, there is evidence in literature for a time factor for pneumonitis with an estimated dose recovery of $0.44 \mathrm{~Gy} /$ day $^{35}$. Since no model is currently available taking this recovery into account when assessing the MLD based risk estimation for pneumonitis, we used the model without adjustment for overall treatment time. Although the current model is based on nMLD, a better predictor of radiotherapy induced lung injury is needed ${ }^{45}$. The prediction should probably not only be based on dosimetric parameters, but should also take into account patientrelated factors, like lung function and smoking habits.

One of the limitations of a modeling study is that there are uncertainties in risk estimates as expressed above. The risk estimates depend on several radiobiological parameters, such as $a / \beta$ and $D_{50}$. We applied literature-based values for these parameters, and therefore we believe that the results for TCP and risk for pneumonitis are the best possible estimates, based on current available data. Clinical studies are needed to verify these results.

Although the results of clinical studies have to be awaited, no major impact on late effects is expected since several authors already have shown that it is safe to escalate dose with regard to toxicity $5,11,23-25$. In contrast to late toxicity, a clinically relevant increase in acute esophagitis is expected escalating the total tumor dose to $79.2 \mathrm{~Gy}$. In a dose escalation study performed by Belderbos et al., and confirmed by other authors, about half of the patients $(48 / 88)$ had grade 1 or 2 acute esophagitis, while no grade 3 
or higher occurred ${ }^{23,24,46-48}$. Since acute esophagitis is thus not dose limiting, when no concurrent chemo-radiation is administered, no constraint for the esophagus was taken into account in this study.

One could argue that the concurrent chemo-radiation is the standard treatment for patients with locally advanced NSCLC, because of improved survival rates ${ }^{9,15,17}$. However, the combined schedules also give rise to rather high toxicity levels $s^{9,15,17,22,46,49}$. Moreover, a large number of patients with co-morbidity cannot profit from concurrent schemes, and we therefore believe that optimizing non-concurrent radiation strategies are still very valuable. These patients may potentially benefit from a combination of radiation dose escalation and acceleration.

Our results suggest that using a personalized maximal tolerable dose scheme, given in twice daily fractions of $1.8 \mathrm{~Gy}$ can increase tumor control probability from $4.5 \%$ for the classic scheme of $60 \mathrm{~Gy}$ in $2.0 \mathrm{~Gy}$ fractions once daily, to $30.9 \%$ for the new scheme. A further improvement in both the prediction of radiation induced lung injury, as well as the prediction of tumor response might give the opportunity to further individualize radiation treatment in NSCLC patients. The results of this in silico trial should be confirmed in a phase II trial. The results of such a trail, ongoing in our department, are awaited. 


\section{References}

1. Le Chevalier T, Arriagada R, Quoix E, et al. Radiotherapy alone versus combined chemotherapy and radiotherapy in nonresectable non-small-cell lung cancer: first analysis of a randomized trial in 353 patients. J Natl Cancer Inst 1991;83:417-423.

2. Qiao X, Tullgren O, Lax I, et al. The role of radiotherapy in treatment of stage I non-small cell lung cancer. Lung Cancer 2003;41:1-11.

3. Saunders M, Dische S, Barrett A, et al. Continuous, hyperfractionated, accelerated radiotherapy (CHART) versus conventional radiotherapy in non-small cell lung cancer: mature data from the randomised multicentre trial. CHART Steering committee. Radiother Oncol 1999;52:137-148.

4. Sause W, Kolesar P, Taylor SI, et al. Final results of phase III trial in regionally advanced unresectable non-small cell lung cancer: Radiation Therapy Oncology Group, Eastern Cooperative Oncology Group, and Southwest Oncology Group. Chest 2000;117:358-364.

5. Belderbos JS, Heemsbergen WD, De Jaeger K, et al. Final results of a Phase I/II dose escalation trial in non-small-cell lung cancer using three-dimensional conformal radiotherapy. Int $\mathrm{J}$ Radiat Oncol Biol Phys 2006;66:126-134.

6. Dillman $\mathrm{RO}$, Seagren $\mathrm{SL}$, Propert $\mathrm{KJ}$, et al. A randomized trial of induction chemotherapy plus high-dose radiation versus radiation alone in stage III non-small-cell lung cancer. $N$ Engl J Med 1990;323:940-945.

7. Kong FM, Ten Haken RK, Schipper MJ, et al. High-dose radiation improved local tumor control and overall survival in patients with inoperable/unresectable non-small-cell lung cancer: Longterm results of a radiation dose escalation study. Int J Radiat Oncol Biol Phys 2005;63:324333.

8. Perez CA, Pajak TF, Rubin P, et al. Long-term observations of the patterns of failure in patients with unresectable non-oat cell carcinoma of the lung treated with definitive radiotherapy. Report by the Radiation Therapy Oncology Group. Cancer 1987;59:1874-1881.

9. Rowell NP, O'Rourke N P. Concurrent chemoradiotherapy in non-small cell lung cancer. Cochrane Database Syst Rev 2004:CD002140.

10. Bradley J. A review of radiation dose escalation trials for non-small cell lung cancer within the Radiation Therapy Oncology Group. Semin Oncol 2005;32:S111-113.

11. Hayman JA, Martel MK, Ten Haken RK, et al. Dose escalation in non-small-cell lung cancer using three-dimensional conformal radiation therapy: update of a phase I trial. J Clin Oncol 2001;19:127-136.

12. Marks LB, Garst J, Socinski MA, et al. Carboplatin/paclitaxel or carboplatin/vinorelbine followed by accelerated hyperfractionated conformal radiation therapy: report of a prospective phase I dose escalation trial from the Carolina Conformal Therapy Consortium. J Clin Oncol 2004;22:4329-4340.

13. Martel MK, Ten Haken RK, Hazuka MB, et al. Estimation of tumor control probability model parameters from 3-D dose distributions of non-small cell lung cancer patients. Lung Cancer $1999 ; 24: 31-37$.

14. Baumann M, Herrmann $T$, Koch R, et al. Continuous hyperfractionated accelerated radiotherapy - weekend less (CHARTWEL) versus conventionally fractionated (CF) radiotherapy in non-smallcell lung cancer (NSCLC): first results of a phase III randomised multicentre trial (ARO 9-71) ECCO 13, 2005. Eur J Cancer 2005;Supplements vol 3:322.

15. Curran WJ, Jr. Evolving chemoradiation treatment strategies for locally advanced non-small-cell lung cancer. Oncology (Williston Park) 2003;17:7-14.

16. Zatloukal P, Petruzelka L, Zemanova $M$, et al. Concurrent versus sequential chemoradiotherapy with cisplatin and vinorelbine in locally advanced non-small cell lung cancer: a randomized study. Lung Cancer 2004;46:87-98.

17. Fournel $P$, Robinet $G$, Thomas $P$, et al. Randomized phase III trial of sequential chemoradiotherapy compared with concurrent chemoradiotherapy in locally advanced non-small-cell lung cancer: Groupe Lyon-Saint-Etienne d'Oncologie Thoracique-Groupe Francais de PneumoCancerologie NPC 95-01 Study. J Clin Oncol 2005;23:5910-5917. 
18. Furuse K, Fukuoka M, Kawahara M, et al. Phase III study of concurrent versus sequential thoracic radiotherapy in combination with mitomycin, vindesine, and cisplatin in unresectable stage III non-small-cell lung cancer. J Clin Oncol 1999;17:2692-2699.

19. Huber RM, Flentje M, Schmidt M, et al. Simultaneous chemoradiotherapy compared with radiotherapy alone after induction chemotherapy in inoperable stage IIIA or IIIB non-small-cell lung cancer: study CTRT99/97 by the Bronchial Carcinoma Therapy Group. J Clin Oncol $2006 ; 24: 4397-4404$.

20. Fowler JF, Tome WA, Fenwick JD, et al. A challenge to traditional radiation oncology. Int $J$ Radiat Oncol Biol Phys 2004;60:1241-1256.

21. Kwa SL, Lebesque JV, Theuws JC, et al. Radiation pneumonitis as a function of mean lung dose: an analysis of pooled data of 540 patients. Int J Radiat Oncol Biol Phys 1998;42:1-9.

22. Wang S, Liao Z, Wei X, et al. Analysis of clinical and dosimetric factors associated with treatment-related pneumonitis (TRP) in patients with non-small-cell lung cancer (NSCLC) treated with concurrent chemotherapy and three-dimensional conformal radiotherapy (3D-CRT). Int J Radiat Oncol Biol Phys 2006;66:1399-1407.

23. Bradley J, Graham MV, Winter K, et al. Toxicity and outcome results of RTOG 9311: a phase III dose-escalation study using three-dimensional conformal radiotherapy in patients with inoperable non-small-cell lung carcinoma. Int J Radiat Oncol Biol Phys 2005;61:318-328.

24. Kong FM, Hayman JA, Griffith KA, et al. Final toxicity results of a radiation-dose escalation study in patients with non-small-cell lung cancer (NSCLC): predictors for radiation pneumonitis and fibrosis. Int J Radiat Oncol Biol Phys 2006;65:1075-1086.

25. Rosenzweig KE, Fox JL, Yorke E, et al. Results of a phase I dose-escalation study using threedimensional conformal radiotherapy in the treatment of inoperable nonsmall cell lung carcinoma. Cancer 2005;103:2118-2127.

26. De Ruysscher D, Wanders S, Minken A, et al. Effects of radiotherapy planning with a dedicated combined PET-CT-simulator of patients with non-small cell lung cancer on dose limiting normal tissues and radiation dose-escalation: a planning study. Radiother Oncol 2005;77:5-10.

27. van Baardwijk A, Bosmans G, Dekker A, et al. Time trends in the maximal uptake of FDG on PET scan during thoracic radiotherapy. A prospective study in locally advanced non-small cell lung cancer (NSCLC) patients. Radiother Oncol 2007;82:145-152.

28. van Der Wel A, Nijsten $S$, Hochstenbag $M$, et al. Increased therapeutic ratio by 18FDG-PET CT planning in patients with clinical CT stage N2-N3M0 non-small-cell lung cancer: a modeling study. Int J Radiat Oncol Biol Phys 2005;61:649-655.

29. ICRU. Prescribing, Recording and Reporting Photon Beam Therapy ICRU Report 50. 1993; ISBN 0-913394-48-3.

30. Abratt RP, Bogart JA, Hunter A. Hypofractionated irradiation for non-small cell lung cancer. Lung Cancer 2002;36:225-233.

31. Graham MV, Pajak TE, Herskovic AM, et al. Phase I/II study of treatment of locally advanced (T3/T4) non-oat cell lung cancer with concomitant boost radiotherapy by the Radiation Therapy Oncology Group (RTOG 83-12): long-term results. Int J Radiat Oncol Biol Phys 1995;31:819825 .

32. Schultheiss TE, Kun LE, Ang KK, et al. Radiation response of the central nervous system. Int J Radiat Oncol Biol Phys 1995;31:1093-1112.

33. De Ruysscher D, Wanders S, van Haren E, et al. Selective mediastinal node irradiation based on FDG-PET scan data in patients with non-small-cell lung cancer: a prospective clinical study. Int J Radiat Oncol Biol Phys 2005;62:988-994.

34. Steel G. Basic Clinical Radiobiology. 3rd ed. London: Hodder Arnold Publications; 2002.

35. Bentzen SM, Saunders MI, Dische S. From CHART to CHARTWEL in non-small cell lung cancer: clinical radiobiological modelling of the expected change in outcome. Clin Oncol ( $R$ Coll Radiol) 2002;14:372-381.

36. Thames HD. An 'incomplete-repair' model for survival after fractionated and continuous irradiations. Int J Radiat Biol Relat Stud Phys Chem Med 1985;47:319-339.

37. Ang KK, Jiang GL, Guttenberger R, et al. Impact of spinal cord repair kinetics on the practice of altered fractionation schedules. Radiother Oncol 1992;25:287-294. 
38. Bentzen SM, Ruifrok AC, Thames HD. Repair capacity and kinetics for human mucosa and epithelial tumors in the head and neck: clinical data on the effect of changing the time interval between multiple fractions per day in radiotherapy. Radiother Oncol 1996;38:89-101.

39. Senan S, De Ruysscher D, Giraud $P$, et al. Literature-based recommendations for treatment planning and execution in high-dose radiotherapy for lung cancer. Radiother Oncol 2004; 71:139-146.

40. Goitein M, Schultheiss TE. Strategies for treating possible tumor extension: some theoretical considerations. Int J Radiat Oncol Biol Phys 1985;11:1519-1528.

41. Lyman JT, Wolbarst AB. Optimization of radiation therapy, III: A method of assessing complication probabilities from dose-volume histograms. Int J Radiat Oncol Biol Phys 1987;13:103-109.

42. Kutcher GJ, Burman C. Calculation of complication probability factors for non-uniform normal tissue irradiation: the effective volume method. Int J Radiat Oncol Biol Phys 1989;16:16231630.

43. Lyman JT. Complication probability as assessed from dose-volume histograms. Radiat Res Suppl 1985;8:S13-19.

44. Knap MM, Bentzen SM, Overgaard J. Late neurological complications after irradiation of malignant tumors of the testis. Acta Oncol 2007;46:497-503.

45. Kocak Z, Borst GR, Zeng J, et al. Prospective assessment of dosimetric/physiologic-based models for predicting radiation pneumonitis. Int J Radiat Oncol Biol Phys 2007;67:178-186.

46. Belderbos J, Heemsbergen W, Hoogeman M, et al. Acute esophageal toxicity in non-small cell lung cancer patients after high dose conformal radiotherapy. Radiother Oncol 2005;75:157164.

47. Takeda K, Nemoto K, Saito H, et al. Dosimetric correlations of acute esophagitis in lung cancer patients treated with radiotherapy. Int J Radiat Oncol Biol Phys 2005;62:626-629.

48. Wu KL, Jiang GL, Liao Y, et al. Three-dimensional conformal radiation therapy for non-small-cell lung cancer: a phase I/II dose escalation clinical trial. Int $J$ Radiat Oncol Biol Phys 2003; $57: 1336-1344$.

49. Bradley J, Deasy JO, Bentzen S, et al. Dosimetric correlates for acute esophagitis in patients treated with radiotherapy for lung carcinoma. Int J Radiat Oncol Biol Phys 2004;58:1106-1113. 


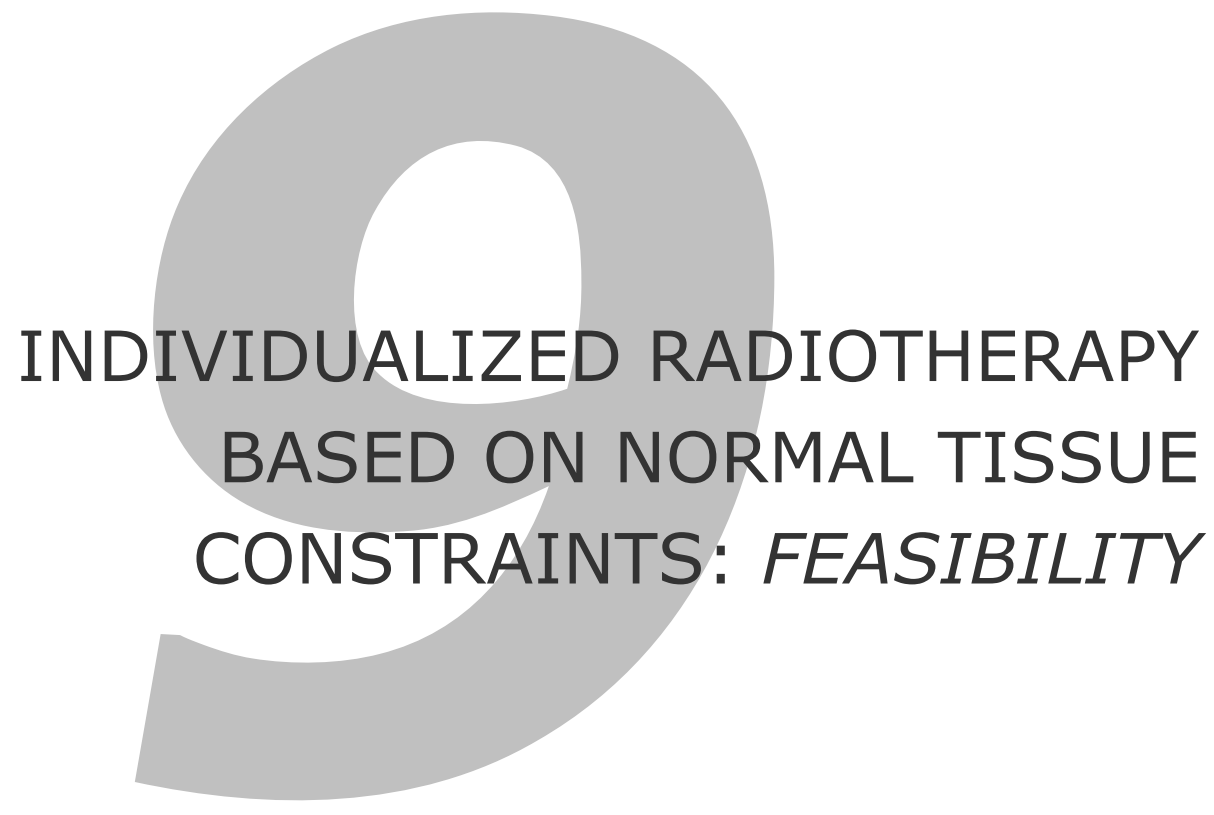

A. van Baardwijk, G. Bosmans, L. Boersma, R. Wanders, A. Dekker, A. C. Dingemans, G. Bootsma, W. Geraedts, C. Pitz, J. Simons, P. Lambin and D. De Ruysscher. Individualized radical radiotherapy of non-small cell lung cancer (NSCLC) based on normal tissue dose constraints: a feasibility study.

Int J Rad Oncol Biol 2008 (in press) 


\section{Abstract}

Purpose: Local recurrence is a major problem after (chemo-)radiation for NSCLC. We hypothesized that for each individual patient, the highest therapeutic ratio could be achieved by increasing the total tumor dose (TTD) to the limits of normal tissues, delivered within 5 weeks. We report the first results of a prospective feasibility trial.

Materials and Methods: Twenty-eight patients with medically inoperable or locally advanced NSCLC, WHO-performance score of $0-1$, and a reasonable lung function $\left(\mathrm{FEV}_{1}>50 \%\right)$ were analyzed. All patients underwent irradiation using an individualized prescribed TTD, based on normal tissue dose constraints (mean lung dose $19 \mathrm{~Gy}$; maximal spinal cord dose $54 \mathrm{~Gy}$ ) up to a maximal TTD of $79.2 \mathrm{~Gy}$, in $1.8 \mathrm{~Gy}$ fractions twice daily. No concurrent chemo-radiation was administered. Toxicity was scored using the CTCAE-criteria. An FDG-PET-CT scan was performed to evaluate (metabolic) response 3 months after treatment.

Results: Mean delivered dose was $63.0 \pm 9.8 \mathrm{~Gy}$. The TTD was most often limited by the MLD (32.1\%) or spinal cord (28.6\%). Acute toxicity was generally mild; only 1 patient experienced grade 3 cough and 1 patient grade 3 dysphagia. One patient $(3.6 \%)$ died of pneumonitis. For late toxicity 2 patients $(7.7 \%)$ had grade 3 cough or dyspnea; none had severe dyshagia. Complete metabolic response was obtained in $44 \%$ (11 of 26 patients). With a median follow-up of 13 months, median overall survival was 19.6 months, with a 1 -year survival of $57.1 \%$.

Conclusions: Individualized maximal tolerable dose irradiation based on normal tissue dose constraints is feasible, and initial results are promising. 


\section{Introduction}

For patients with inoperable and/or locally advanced (stage III) non-small-cell lung cancer (NSCLC), radiotherapy alone or combined with chemotherapy is the principal choice of treatment. Conventionally, radiation doses of 60 to $66 \mathrm{~Gy}$ are delivered. However, these schedules give rise to local failure in the majority of patients resulting in 2-year local tumor control rates of less than $20 \%^{1,2}$. Several approaches have therefore been applied to improve local control, including increasing the radiation dose and/or the concomitant administration of chemotherapy ${ }^{1,3-6}$. In several randomized studies higher locoregional control rate was associated with an improved survival ${ }^{7-9}$. However, even with concurrent chemo-radiation local relapse still occurs in about one third of the patients as their initial site of failure ${ }^{8-10}$. Moreover, concurrent schedules give rise to increased levels of toxicity ${ }^{6,10,11}$ and therefore are not applicable to patients with co-morbidity ${ }^{12}$. We therefore believe that optimizing non-concurrent chemoradiation strategies are still very valuable.

Several investigators have reported radiation dose escalation studies in $\mathrm{NSCLC}^{3-5}$. Delivery of higher doses up to a total tumor dose (TTD) of $94.5 \mathrm{~Gy}$ was associated with improved local control and overall survival ${ }^{3}$. Using a mathematical model it was suggested that a dose of $84 \mathrm{~Gy}$ might be needed to achieve a $50 \%$ tumor control probability (TCP) in patients with locally advanced NSCLC ${ }^{13}$. However, the TTD that can be given is mainly limited by damage to normal tissues, such as lung, esophagus and spinal cord. Dosimetric factors, such as the mean lung dose (MLD), have been correlated with the probability to develop radiation pneumonitis ${ }^{14-16}$. As a consequence, phase I/II studies were initiated to evaluate the feasibility for dose escalation based on bins using different modes to assess lung injury in the several risk groups ${ }^{3,17,18}$. These trials supported the feasibility of this approach. The dose that can be delivered safely is obviously related to the radiation technique and the imaging modalities used to delineate the target volume. Earlier, we showed that the use of ${ }^{18} \mathrm{~F}$-fluoro-2-deoxyglucose (FDG) Positron Emission Tomography-Computer Tomography (PET-CT) scans for target volume delineation in NSCLC decreased radiation exposure of the esophagus and lung, and thus allowed for significant radiation dose escalation and an estimated increase in $\mathrm{TCP}^{19,20}$. In prospective studies, selective irradiation of only FDG-PET positive mediastinal nodes, did not increase the incidence of isolated nodal failures ${ }^{3,21}$. In addition to the mentioned factors, overall treatment time (OTT) of radiotherapy is considered an important factor for local tumor control ${ }^{2}$.

In most dose escalation studies patients have been stratified to escalating radiation dose levels depending on assignment to different risk groups for the development of radiation pneumonitis. We hypothesized that the best achievable radiation schedule would include the highest TTD based on individualized normal tissue dose constraints, delivered in an OTT of less than 5 weeks ${ }^{22}$. The theoretical gain of such a scheme was estimated to be $25 \%$ in tumor control probability compared with a classical scheme of $60 \mathrm{~Gy}$ in $2 \mathrm{~Gy}$ fractions in 6 weeks $^{23}$. The main objective of this study is to evaluate the feasibility of an individualized maximal tolerable dose radiation prescription, using an accelerated schedule, in patients with inoperable and/or locally advanced NSCLC. Results of the 29 patients enrolled in this prospective feasibility study with respect to toxicity and outcome are reported here. 


\section{Materials and Methods}

\section{Patient eligibility}

Patients eligible for this study had medically inoperable (stage I or II) or locally advanced (stage III) histologically or cytologically confirmed NSCLC. Inclusion criteria were: WHO-performance sore of 0 or 1 , a weight loss of less than $10 \%$ in 6 months and a reasonable lung function (a forced expiratory volume in 1 second $\left(\mathrm{FEV}_{1}\right) \geq 50 \%$ of predicted value and a carbon monoxide diffusing capacity (DLCO) $\geq 50 \%$ ). No concurrent chemo-radiation was allowed. Induction chemotherapy was allowed for stage III patients and consisted of three courses of Gemcitabine $\left(1250 \mathrm{mg} / \mathrm{m}^{2}\right.$ on day 1 and 8$)$, in combination with Cisplatin $\left(75 \mathrm{mg} / \mathrm{m}^{2}\right.$ on day 1$)$ or Carboplatin administered at the Calvert formula for a target area concentration curve (AUC) of $5 \mathrm{mg} / \mathrm{ml}$. min (day 1$)^{24}$. Cycles were repeated every 21 days, and standard dose-reduction rules were applied if indicated. An interval between the last course of chemotherapy and start of radiotherapy of at minimum 14 days was mandatory. No previous radiotherapy to the chest was allowed. Patients were not eligible if they had any other concomitant malignant disease, recent severe cardiac disease or active peptic esophagitis.

\section{Radiotherapy treatment planning}

In all patients a treatment planning PET-CT scan before the start of radiation was obtained in radiotherapy position on a dedicated PET-CT-simulator with both arms above the head and using a laser marker system. The PET-CT scanner used was a Biograph $^{\text {TM }}$ (SOMATOM $^{\circledR}$ Sensation 16 with an ECAT ACCEL PET scanner, Siemens AG, Forchheim, Germany). Patients fasted for at least 6 hours before the examination. ${ }^{18} \mathrm{~F}$-fluorodeoxyglucose (MDS Nordion, Liège, Belgium) was injected intravenously and followed by physiologic saline $(10 \mathrm{ml})$. The injected total activity of FDG depended on the patient weight: (weight*4+20) MBq. After a rest period of 45 minutes images were acquired. The CT-scan performed was a spiral CT-scan of the whole thorax with intravenous contrast. The PET images were acquired in 5-minute bed positions, whereas 2 or 3 positions were needed to scan the thoracic region.

Delineation based on fused PET-CT images was performed by the radiation oncologist using a standard clinical delineation protocol. The protocol included fixed window-level (W-L) settings of both CT (lung W 1700; L -300 , mediastinum W 600; L 40) and PET scan (W 30000; L 15000) to be used for delineation. For all patients a Gross Tumor Volume (GTV) was defined based on FDG PET-CT data. The total tumor volume consisted of the primary tumor (GTV-1), which is the (post-chemotherapy) CT based volume (lung window), and the initial PET-positive lymph nodal areas (GTV-2). PET negative nodes proven to be malignant on mediastinoscopy or transesophageal/transbronchial fine needle aspiration were also included in the GTV. For the final delineation of the lymph nodal areas, the CT scan provided a better contrast with surrounding (fat) tissue and was mainly used for the determination of the border of the lymph node station. No elective hilar of mediastinal irradiation was carried out. The Clinical Target Volume (CTV) was defined as the GTV with a margin of $5 \mathrm{~mm}$, whereas another $10 \mathrm{~mm}$ margin for the primary tumor and $5 \mathrm{~mm}$ for the lymph nodal areas was added for the Planning Target Volume (PTV). 
Contouring of the lungs was done automatically by the treatment planning system (XiO, Computer Medical System Inc., St. Louis, Missouri, USA). For the calculation of the MLD, the volume of both lungs minus the GTV was considered ${ }^{25}$. The spinal cord was drawn throughout the whole CT scan and was considered to be at the inner margin of the bony spinal canal. Both normal tissue contours as well as target volumes were outlined on each slice.

A three-dimensional conformal treatment plan using three to six coplanar $10 \mathrm{MV}$ photon fields, was calculated according to the International Commission on Radiation Units and Measurements (ICRU) guidelines ${ }^{26}$. Calculations were performed using a XiO treatment planning system with a fast fourier transform (FFT) convolution algorithm for inhomogeneity corrections. Patients were irradiated on a linear accelerator (Elekta SL 15, Crawley, United Kingdom). Treatment verification was performed using electronic portal imaging device (EPID) measurements ${ }^{27}$. A shrinking action level protocol was used to control setup errors. The EPID images were also used for in vivo dosimetry measurements.

\section{Treatment description}

All patients enrolled in this study underwent irradiation using an individualized prescribed maximal tolerated dose (MTD). The TTD was based on normal tissue constraints instead of a classic fixed prescribed dose. The radiation dose was individually escalated until a dose-limiting normal tissue constraint was reached. For the lungs, a

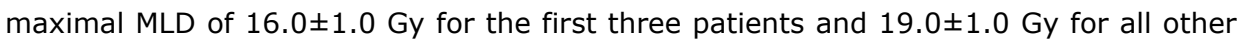
patients was allowed, accepting a theoretical probability of grade 2 pneumonitis (Radiation Therapy Oncology Group; RTOG-criteria) of at maximum $20 \%{ }^{15}$. For the spinal cord a maximal dose of $54.0 \pm 0.5 \mathrm{~Gy}$, for the main bronchi (when both main bronchi were in the high-dose region) a maximum of $70.2 \mathrm{~Gy}$ and for the plexus brachialis a dose constraint of $61.2 \mathrm{~Gy}$ was applied ${ }^{28-31}$. No specific dose constraint for the esophagus was used, because acute esophagitis was not considered to be doselimiting when radiation alone was delivered ${ }^{11}$. Based on the results of Marks et al. we accepted a maximal allowed TTD of $79.2 \mathrm{~Gy}^{31}$. Radiotherapy was delivered twice daily, with a fraction size of $1.8 \mathrm{~Gy}$, because of the relative sparing of normal tissues ${ }^{23}$. An interval of at least 8-hours between the two fractions was respected.

\section{Toxicity and tumor response}

Acute toxicity was scored before start of radiotherapy (baseline) and weekly during radiation treatment. After the last fraction patients were followed up weekly until acute toxicity decreased and afterwards at 2-month intervals, consisting of history and physical examination. Acute and late toxicity was scored according to Common Terminology Criteria for Adverse Events v3.0 (CTCAE) (Table 9.1). A post-treatment FDG-PET-CT scan was performed to evaluate metabolic response on PET according to European Organization for Research and Treatment of Cancer (EORTC) criteria and response on CT according to Response Evaluation Criteria in Sold Tumours (RECIST) criteria $^{32,33}$. This PET-CT scan was performed 70 days after the last fraction of radiotherapy, since it has been shown that metabolic response on PET assesed at this time point is related to overall survival ${ }^{34,35}$. 


\begin{tabular}{|c|c|c|c|c|c|}
\hline Grade & 1 & 2 & 3 & 4 & 5 \\
\hline \multicolumn{6}{|l|}{ Acute toxicity } \\
\hline Cough & $\begin{array}{l}\text { Symptomatic, } \\
\text { non-narcotic } \\
\text { medication only } \\
\text { indicated }\end{array}$ & $\begin{array}{l}\text { Symptomatic } \\
\text { and narcotic } \\
\text { medication } \\
\text { indicated }\end{array}$ & $\begin{array}{l}\text { Symptomatic } \\
\text { and significantly } \\
\text { interfering with } \\
\text { sleep or ADL }\end{array}$ & & \\
\hline Dyspnea & $\begin{array}{l}\text { Dyspnea on } \\
\text { exertion, but can } \\
\text { walk } 1 \text { flight of } \\
\text { stairs without } \\
\text { stopping }\end{array}$ & $\begin{array}{l}\text { Dyspnea on } \\
\text { exertion but } \\
\text { unable to walk } 1 \\
\text { flight of stairs or } \\
1 \text { city block } \\
\text { without stopping }\end{array}$ & $\begin{array}{l}\text { Dyspnea with } \\
\text { ADL }\end{array}$ & $\begin{array}{l}\text { Dyspnea at rest; } \\
\text { intuba- } \\
\text { tion/ventilator } \\
\text { indicated }\end{array}$ & Death \\
\hline Dysphagia & $\begin{array}{l}\text { Symptomatic, } \\
\text { able to eat } \\
\text { regular diet }\end{array}$ & $\begin{array}{l}\text { Symptomatic } \\
\text { and altered } \\
\text { eat- } \\
\text { ing/swallowing, } \\
\text { iv fluids }<24 \text { hrs }\end{array}$ & $\begin{array}{l}\text { Severely altered } \\
\text { eat- } \\
\text { ing/swallowing; } \\
\text { iv fluids, tube } \\
\text { feeding or TPN } \\
\geq 24 \text { hrs }\end{array}$ & $\begin{array}{l}\text { Life-threating } \\
\text { consequences }\end{array}$ & Death \\
\hline Dermatitis & $\begin{array}{l}\text { Faint erythema } \\
\text { or dry desqua- } \\
\text { mation }\end{array}$ & $\begin{array}{l}\text { Moderate-briske } \\
\text { erythema; } \\
\text { patchy moist } \\
\text { desquamation, } \\
\text { mostly confined } \\
\text { to skin folds and } \\
\text { creases; } \\
\text { moderate edema }\end{array}$ & $\begin{array}{l}\text { Moist desquama- } \\
\text { tion other than } \\
\text { skin folds and } \\
\text { creases; } \\
\text { bleeding induced } \\
\text { by minor trauma } \\
\text { or abrasion }\end{array}$ & $\begin{array}{l}\text { Skin necrosis or } \\
\text { ulceration of tull } \\
\text { thicknees } \\
\text { dermis; } \\
\text { spontaneous } \\
\text { bleeding from } \\
\text { involved site }\end{array}$ & Death \\
\hline \multicolumn{6}{|l|}{ Late toxicity } \\
\hline Pneumonitis & $\begin{array}{l}\text { Asymptomatic, } \\
\text { radiographic } \\
\text { findings only }\end{array}$ & $\begin{array}{l}\text { Symptomatic, } \\
\text { not interfering } \\
\text { with ADL }\end{array}$ & $\begin{array}{l}\text { Symptomatic, } \\
\text { interfering with } \\
\mathrm{ADL} ; \mathrm{O} 2 \\
\text { indicated }\end{array}$ & $\begin{array}{l}\text { Life-threating ; } \\
\text { ventilatory } \\
\text { support indicated }\end{array}$ & Death \\
\hline $\begin{array}{l}\text { Pulmonary fibrosis } \\
\text { (radiographic changes) }\end{array}$ & $\begin{array}{l}\text { Minimal } \\
\text { radiographic } \\
\text { findings (or } \\
\text { patchy or } \\
\text { bibasilar } \\
\text { changes) with } \\
\text { estimated } \\
\text { radiographic } \\
\text { proportion of } \\
\text { total lung } \\
\text { volume that is } \\
\text { fibrotic of }<25 \%\end{array}$ & $\begin{array}{l}\text { Patchy or bi- } \\
\text { basalar changes } \\
\text { with estimated } \\
\text { radiographic } \\
\text { total lung } \\
\text { volume that is } \\
\text { fibrotic of } 25- \\
<50 \%\end{array}$ & $\begin{array}{l}\text { Dense or wide- } \\
\text { spread in- } \\
\text { flitrates/consolid } \\
\text { ation with } \\
\text { estimated } \\
\text { radiographic } \\
\text { proportion of } \\
\text { total lung } \\
\text { volume of } 50- \\
<75 \%\end{array}$ & $\begin{array}{l}\text { Estimated } \\
\text { radiographic } \\
\text { proportion of } \\
\text { total lung } \\
\text { volume that is } \\
\text { fibrotic is } \geq 75 \% \text {; } \\
\text { honeycombing }\end{array}$ & Death \\
\hline
\end{tabular}

\section{Statistical analysis}

The SPSS software (SPSS for Windows, version 15.0, SPSS Inc., Chicago, IL) was used for statistical analysis. Accepting a maximal incidence of $20 \%$ of grade 3 pneumonitis as primary endpoint (according to CTCAE v3.0-criteria), a number of 29 patients was calculated to be included. If at maximum 3 out of 29 patients $(10.5 \%$, upper limit of one-sided 95\% confidence interval: $19.7 \%$ ) would develop grade 3 pneumonitis the schedule would be considered to be safe for the primary endpoint. Results are expressed as mean \pm standard deviation (SD), unless otherwise stated. Crude incidences of pulmonary complaints (cough and dyspnea), esophageal dysphagia and skin dermatitis (maximum score) according to CTCAE v3.0-criteria were calculated for 
the acute phase (90 days from start of radiotherapy) and pneumonitis and pulmonary fibrosis for the late phase (>90 days from start of radiotherapy). Progression-free survival (time between start radiotherapy and first evidence of local or distant recurrence, in case of death without recurrence patients were censored) and overall survival (time between start of radiotherapy and death due to any cause) were calculated according to the Kaplan-Meier method. Patients still alive at the time of analysis were censored at the last day of follow-up. Follow-up data from all patients were collected until April 1, 2007. At this date, 13 months after the inclusion of the last patient, data were analyzed.

\section{Ethics}

This study was reviewed and approved by the local Medical Ethical Committee, according to the Dutch law and regulations. All patients gave written informed consent before inclusion.

\section{Results}

\section{Patient and treatment characteristics}

Between December 2004 and March 2006, a total of 29 patients (20 men and 9 women) were enrolled in the study. One patient was not treated according to the study protocol, but received inappropriately our standard protocol, a classical once daily scheme of $60 \mathrm{~Gy}$ in 30 fractions in 6 weeks, and was excluded from further analysis. Patient and tumor characteristics of all 28 patients are listed in Table 9.2. Concerning lymph nodal status 7 patients had cNO, 1 patient cN1, 11 patients CN2 ( 3 pathologically confirmed) and 9 patients cN3 (4 pathologically confirmed).

Median follow-up for the total group of patients was 13.9 months (range $0.8-24.2$ months), with a median follow-up of 17.2 months (range 9.6-23.0 months) for patients still alive at the time of analysis.

Twenty-four patients (86\%) received induction chemotherapy, consisting of three courses of Gemcitabine in combination with Cisplatin or Carboplatin. Seventeen out of the 24 patients $(70.8 \%)$ who received chemotherapy had a partial response after three cycles, with no complete remissions observed. The remainder showed stable disease. The median interval between the last day of chemotherapy and the first day of radiotherapy was 35 days.

Mean TTD was $63.0 \pm 9.8 \mathrm{~Gy}$ (range 46.9-79.2 Gy) in $1.8 \mathrm{~Gy}$ fractions twice daily in a mean OTT of $24 \pm 4$ days. In 5 out of 28 patients (17.9\%, all stage IIIB) the maximal allowed TTD of 79.2 Gy could be delivered. 


\begin{tabular}{|c|c|c|c|}
\hline \multicolumn{4}{|l|}{ Characteristic } \\
\hline Mean age (years) & & 65.0 & $(50-80)$ \\
\hline \multirow[t]{2}{*}{ Gender } & Male & 19 & (68) \\
\hline & Female & 9 & $(32)$ \\
\hline \multirow[t]{2}{*}{ WHO performance score } & 0 & 20 & (71) \\
\hline & 1 & 8 & (29) \\
\hline \multirow[t]{5}{*}{ Clinical stage } & $I$ & 2 & (7) \\
\hline & $I I$ & 0 & $(0)$ \\
\hline & IIIA & 7 & (25) \\
\hline & IIIB & 19 & (68) \\
\hline & $\begin{array}{l}\text { Squamous cell } \\
\text { carcinoma }\end{array}$ & 9 & (32) \\
\hline \multirow{2}{*}{ Histology } & Adenocarcinoma & 5 & (18) \\
\hline & Large cell carcinoma & 14 & (50) \\
\hline \multirow[t]{2}{*}{ Induction chemotherapy } & Yes & 24 & $(86)$ \\
\hline & No & 4 & (14) \\
\hline \multirow[t]{2}{*}{ Mean GTV in cc } & Primary tumor & 93.9 & $(0.4-660.2)$ \\
\hline & Lymphnodes & 25.2 & $(0-155.0)$ \\
\hline Mean total tumor load in cc & & 119.2 & $(12.2-674.4)$ \\
\hline Mean TTD in Gy & & 63.0 & $(46.8-79.2)$ \\
\hline Mean MLD in Gy & & 16.6 & $(6.8-20.8)$ \\
\hline Mean Maximal spinal cord in Gy & & 46.3 & $(6.8-57.3)$ \\
\hline Mean OTT in days & & 24.4 & $(17-31)$ \\
\hline
\end{tabular}

Only 1 patient (3.6\%) received a dose below $50 \mathrm{~Gy}(46.9 \mathrm{~Gy}$ ) and 11 patients $(39.2 \%)$ received a dose between 50 and $60 \mathrm{~Gy}$. The maximal TTD was most often limited by the MLD $(32.1 \%)$, and secondly by the spinal cord dose $(28.6 \%)$, or both $(10.7 \%)$. In 3 patients $(10.7 \%)$ other structures were dose limiting: the main bronchii and plexus brachialis. In 3 patients, a major protocol violation was encountered. Two patients with limited lung function ( $\mathrm{FEV}_{1}<50 \%$ of predicted value and/or a DLCO $<50 \%$ ) were included in the study, and in one patient the TTD was not escalated up to the maximal allowed dose. Eight minor protocol violations were observed, including 6 patients with a maximal spinal cord dose of more than 54.5 Gy (range 55.1-57.1 Gy) and 2 patients with an MLD above 20 Gy (20.7 Gy and 20.8 Gy, respectively).

\section{Toxicity}

Acute toxicity during the first 3 months was scored for all 28 patients (Figure 9.1). Most patients experienced only mild acute toxicity. Regarding pulmonary complaints, almost $70 \%$ of patients $(n=19)$ experienced increased cough, but only 6 patients required narcotic medication (e.g. codeine) during fractionated radiotherapy. One patient $(3.6 \%)$ developed grade 3 cough toxicity. Dyspnea was less frequently observed; only 4 patients $(14.3 \%)$ had a grade 1 or 2 dyspnea toxicity score. One patient (3.6\%) died 51 days after radiotherapy due to pneumonitis (grade 5 toxicity). Acute esophageal toxicity was generally mild, although most patients $(85.7 \%)$ had symptomatic dysphagia grade 1 or 2 . Only 1 patient (3.6\%) developed severe swallowing symptoms (grade 3 ), requiring temporarily tube feeding. No grade 2 or higher dermatitis was observed during or after radiotherapy. 
Two patients died within 90 days. One patient died of a myocardial infarction within one week after the last fraction of radiotherapy (tumor in right upper lobe, mean heart dose $20.9 \mathrm{~Gy}$ ). The other patient died of an acute respiratory distress syndrome based on pneumonitis, as described. The total dose prescribed for this last patient with an impaired lungfunction ( $\mathrm{FEV}_{1} 104 \%$ and DLCO 38\%) was 61.2 Gy with an MLD of $15.5 \mathrm{~Gy}$.

With a median follow-up of 13.9 months, 26 patients were available to assess late toxicity. Seventy days after radiotherapy radiographic changes were shown in $92 \%$ of patients $(n=24)$ on CT ( $73 \%$ in $<25 \%$ and $19 \%$ in $>25 \%$ of the lungs). However, only one third of patients (10 of 26 patients) had clinical symptoms ( $\geq$ grade 2 pneumonitis). Two patients $(7.7 \%)$ had severe symptoms of cough and dyspnea interfering with activities of daily living (grade 3 pneumonitis), whereas in 4 patients corticosteroids (in 3 out of 4 inhalation corticosteroids only) were prescribed. Regarding dysphagia, 3 patients $(11.5 \%)$ had swallowing symptoms 2 to 9 months after radiotherapy. Two patients had mild symptoms, but were able to eat a regular diet. One patient with grade 2 dysphagia developed simultaneous a locoregional recurrence, which might explain the complaints.

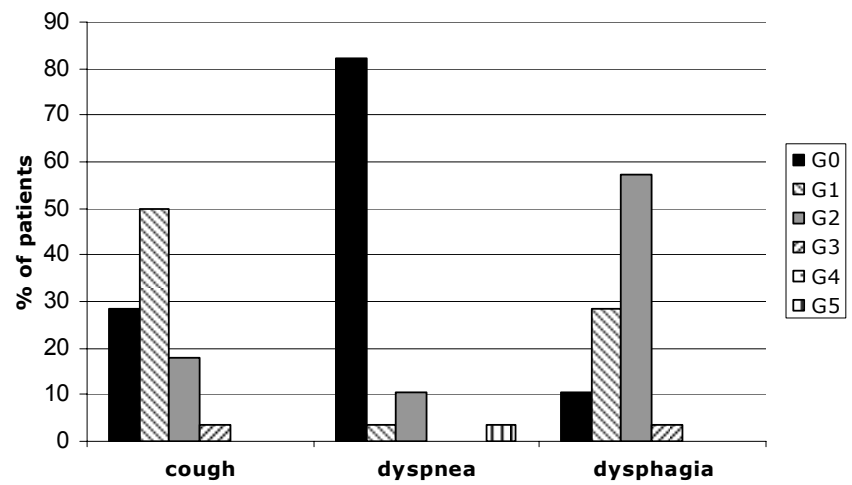

a

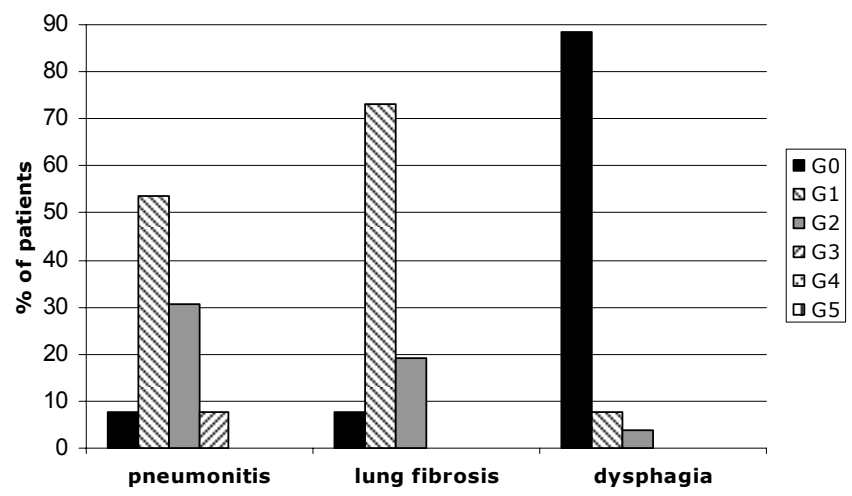

$b$

Figure 9.1 Maximum toxicity score of acute toxicity within 90 days (a) and late toxicity after 90 days (b) according to CTCAE-criteria version 3.0 (Table 9.1). 


\section{Outcome}

At the time of analysis, 15 patients (53.6\%) had died. The median overall survival was 19.6 months (range 0.8-26.3 months) with an estimated 1-year and 2-year survival rates of $57.1 \%$ and $47.1 \%$ respectively, as shown in Figure 9.2 a.

At a median follow-up of 13 months, the incidence of loco-regional tumor progression was $35.7 \%(n=10)$. Only 2 patients $(7.1 \%)$ had isolated local recurrence as first site of relapse, both showing an in-field recurrence. There was no isolated nodal recurrence. In 4 of 10 patients loco-regional progression was simultaneously (within 1 month) with distant metastases. In 3 out of these 4 patients, loco-regional progression was not located within the original radiation fields. About $40 \%$ of patients (11 of 28 ) developed distant metastases during follow-up. Median progression free survival was 10.2 months (range 0.8-23.6 months) with estimated 1-year and 2-year progression free survival rates of $53.8 \%$ and $27.1 \%$, respectively (Figure $9.2 \mathrm{~b}$ ).

\section{PET and CT response}

A follow-up PET-CT scan was performed in 26 out of 28 patients 70 days after radiotherapy to evaluate tumor response. Two patients died within 70 days after radiotherapy and in 1 patient only CT data were available, due to a technical problem with the PET scanner. The PET-CT showed in 15 patients $(60 \%)$ a metabolic response, consisting of a complete metabolic response in 11 patients (44\%) and a partial metabolic response in 4 patients $(16 \%)$. Three patients $(12 \%)$ showed stable disease, while $7(28 \%)$ patients showed progressive disease. According to the RECIST criteria 13 patients (50\%) showed a response, consisting of one (4\%) complete response and $12(46 \%)$ partial responses (response on PET vs. CT; $p<0.01)$. In all 7 patients $(28 \%)$ showing progressive metabolic disease on PET, this was confirmed on CT. All these patients showed metastatic disease, in three combined with local or loco-regional progression.

\section{Discussion}

The main purpose of this study was to evaluate the feasibility of individualized dose prescription based on normal tissue dose constraints, basically an MLD of 19 Gy for lung and a maximal dose of $54 \mathrm{~Gy}$ for the spinal cord. The radiation dose was delivered in twice-daily fractions of $1.8 \mathrm{~Gy}$ with an 8 -hour interval between fractions. First results of this prospective trial are promising, showing that this approach can be applied safely with acceptable toxicity. Mainly mild acute lung toxicity was observed; although in 1 patient pneumonitis was the cause of death shortly after radiotherapy. Acute esophageal toxicity was generally mild. In a dose escalation study performed by Belderbos et al., about half of the patients $(48 / 88)$ had grade 1 or 2 acute esophagitis ${ }^{11}$. Similar esophageal toxicity results were observed by Kong and colleagues ${ }^{18}$. We encountered a greater percentage ( $86 \%$ ) of symptoms of dysphagia grade 1 or 2 during radiotherapy. About half of the patients had problems swallowing and therefore a change in diet (e.g. soft diet). However, only 1 patient (3.6\%) developed severe swallowing symptoms (grade 3 ), requiring tube feeding. No patient had an interruption of radiation treatment due to dysphagia symptoms. 

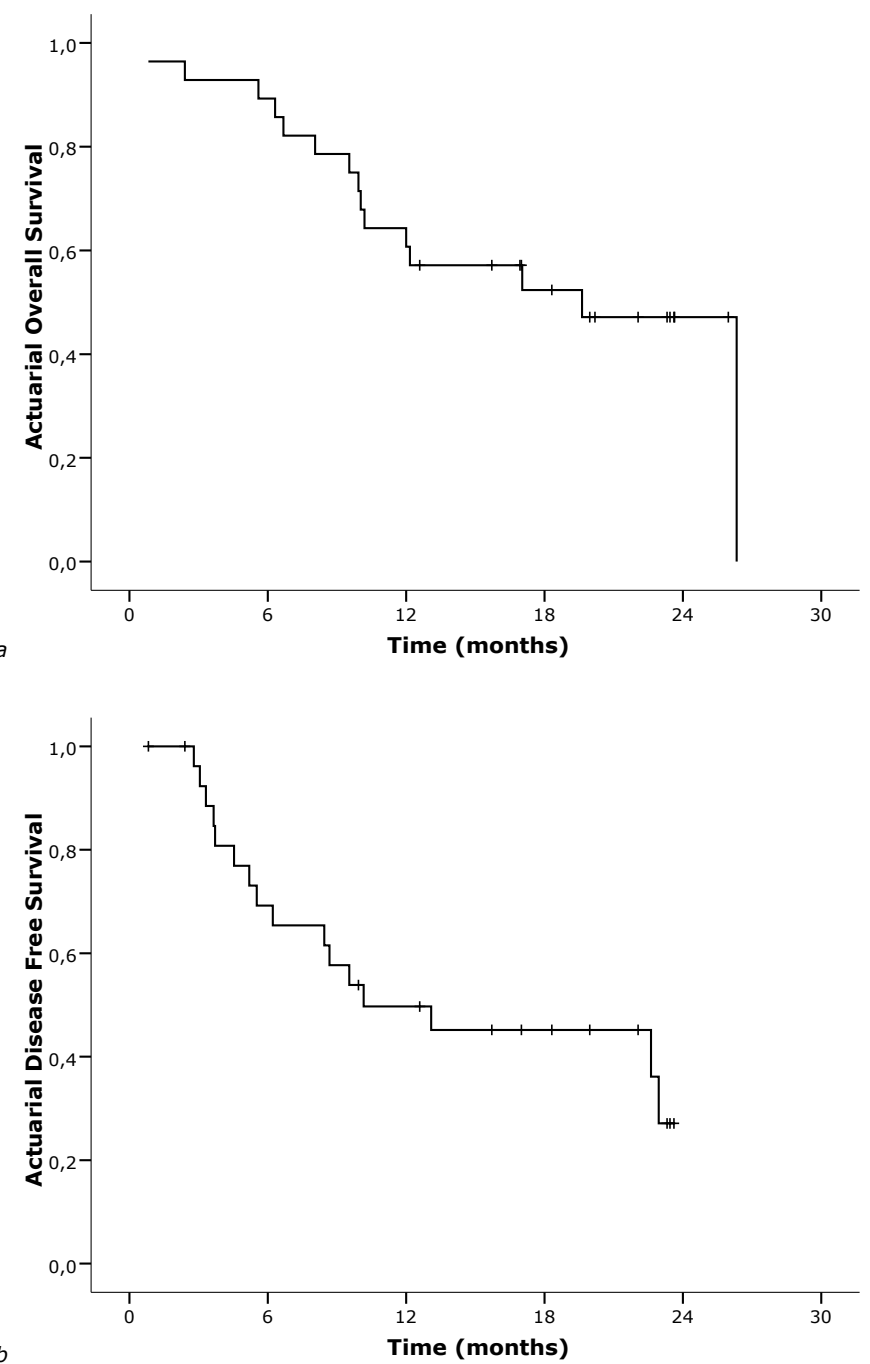

Figure 9.2 Actuarial overall survival (a) and progression free survival (b). Abbreviations: $R T=$ radiotherapy.

The greater proportion of esophageal complaints might be explained by our accelerated schedule, as Saunders et al. found similar results in the Continous Hyperfractionated Accelerated Radiation (CHART) study ${ }^{36}$. In our study acute esophagitis was not dose limiting. Therefore, our policy not to include a specific dose constraint for the esophagus, when no concurrent chemoradiation was administered, is supported by these results.

Radiation pneumonitis can be scored using different toxicity scoring systems, resulting in a large heterogeneity in literature. We used the CTCAE v3.0 scoring system, while other authors used the Southwest Oncology Group (SWOG) criteria ${ }^{3,18}$. These two scoring methods differ, e.g. in their description of grade 2 radiation pneumonitis 
(SWOG grade 2, oral corticosteroids prescription; CTCAE grade 2, symptomatic, not interfering with $A D L)$. Clinically significant radiation pneumonitis is usually seen in 13 to $37 \%$ of patients treated for lung cancer with radical radiotherapy ${ }^{37}$. In our study we observed in 10 of 26 patients at risk (38.4\%) a clinical (CTCAE v3.0 $\geq$ grade 2) pneumonitis. Most patients had mild symptoms, not interfering with ADL (30.8\%), whereas only 1 patient (4\%) needed oral steroid prescription (grade 2 according to SWOG criteria). Two of the 10 patients $(7.7 \%)$ had a grade 3 pneumonitis (CTCAE v3.0), indicating symptomatic pneumonitis, interfering with ADL and/or oxygen indicated. These results are in line with the results of other dose-escalation studies ${ }^{3,18,36}$. Kong et al. found minimal symptoms in $28.4 \%$ of patients, while $11 \%$ needed corticosteroid treatment and $5 \%$ had grade 3 pneumonitis (SWOG criteria) ${ }^{18}$. Similar results were observed by Belderbos et al. ${ }^{3}$. Moreover, our results are below the maximal acceptable incidence of $20 \%$ of grade 3 pneumonitis we used as primary endpoint.

Previous studies have shown that local tumor control was dependent on the radiation dose $^{3,4,38}$. A mean tumor dose of $63.0 \mathrm{~Gy}$ (range 46.9-79.2 Gy) in a mean OTT of 24 days could be delivered without undue toxicity. The present schedule is biologically equivalent to approximately 80 Gy delivered in once-daily fractions of 2 Gy and the side-effects are within range as predicted in an in silico trial by our group ${ }^{23}$. In our study the maximal allowed TTD of 79.2 Gy could be prescribed in 5 patients (18\%). We observed only 2 isolated local recurrences (7\%), while the incidence of loco-regional tumor progression was $35.7 \%(n=10)$. In 4 patients loco-regional progression was simultaneous detected with distant metastases. Moreover, there was no isolated nodal recurrence, confirming that it is safe to apply selective nodal irradiation based on PET$\operatorname{scan}^{3,21}$. Thus, although one third of all patients $(n=10)$ received 54 Gy or less because of normal tissue dose constraints and a mean dose of $63.0 \mathrm{~Gy}$ could be given in a short OTT, our results are similar to the literature, in which much higher doses were required to obtain the same local tumor control ${ }^{3,39}$. This confirms the therapeutic gain that can be expected by reducing the OTT, e.g. applying an accelerated schedule, compared with conventionally fractionated schedules. The present schedule thus allows delivery of high biological radiation doses in a less selected patient population. In addition, a large proportion of our study patients usually would not be considered for doseescalation studies or even radical radiotherapy, due to tumor localization close to the spinal cord, resulting in inclusion of the spinal cord in the PTV and/or inclusion of large lung volumes.

Although not the aim of this study, we observed a promising outcome, with a 1-year survival rate of $57 \%$ and an estimated 2 -year survival rate of $47 \%$. A median overall surivival of almost 20 months is in line with other dose-escalation study results ${ }^{2-4}$. However, we could achieve this with lower total radiation doses given in an accelerated schedule and in patients otherwise not to be considered for high dose radiotherapy. Interestingly, the present results as well as those of others suggest that overall survival rates could be achieved with sequential chemotherapy and high-dose radiation schedules that are similar to concurrent chemoradiation schemes, but with less acute toxicity ${ }^{8,9,40-42}$. This does not come as a surprise, since the increased survival of patients undergoing concurrent chemoradiation is caused by a better local tumor control and not a decreased incidence of metastatic disease ${ }^{7-9}$. 
Because a large proportion of patients with lung cancer has significant co-morbidity ${ }^{12}$, they are excluded from concurrent chemo-radiation schemes. We therefore believe that optimizing non-concurrent radiation strategies is still very valuable. These patients may potentially benefit from an individualized prescription of radiation treatment given in a schedule that combines the principles of dose escalation and acceleration.

In conclusion, individualized maximal tolerable dose irradiation based on normal tissue dose constraints is feasible, and first results for 28 patients seem promising. A phase II trial is ongoing in our department to further assess the gain of this individualized radiation treatment. 


\section{References}

1. Le Chevalier T, Arriagada R, Quoix E, et al. Radiotherapy alone versus combined chemotherapy and radiotherapy in nonresectable non-small-cell lung cancer: first analysis of a randomized trial in 353 patients. J Natl Cancer Inst 1991;83:417-423.

2. Saunders M, Dische S, Barrett A, et al. Continuous, hyperfractionated, accelerated radiotherapy (CHART) versus conventional radiotherapy in non-small cell lung cancer: mature data from the randomised multicentre trial. CHART Steering committee. Radiother Oncol 1999;52:137-148.

3. Belderbos JS, Heemsbergen WD, De Jaeger K, et al. Final results of a Phase I/II dose escalation trial in non-small-cell lung cancer using three-dimensional conformal radiotherapy. Int $J$ Radiat Oncol Biol Phys 2006;66:126-134.

4. Kong FM, Ten Haken RK, Schipper MJ, et al. High-dose radiation improved local tumor control and overall survival in patients with inoperable/unresectable non-small-cell lung cancer: longterm results of a radiation dose escalation study. Int J Radiat Oncol Biol Phys 2005;63:324333.

5. Perez CA, Pajak TF, Rubin P, et al. Long-term observations of the patterns of failure in patients with unresectable non-oat cell carcinoma of the lung treated with definitive radiotherapy. Report by the Radiation Therapy Oncology Group. Cancer 1987;59:1874-1881.

6. Rowell NP, O'Rourke N P. Concurrent chemoradiotherapy in non-small cell lung cancer. Cochrane Database Syst Rev 2004:CD002140.

7. Schaake-Koning C, van den Bogaert W, Dalesio O, et al. Effects of concomitant cisplatin and radiotherapy on inoperable non-small-cell lung cancer. N Engl J Med 1992;326:524-530.

8. Zatloukal $P$, Petruzelka L, Zemanova $M$, et al. Concurrent versus sequential chemoradiotherapy with cisplatin and vinorelbine in locally advanced non-small cell lung cancer: a randomized study. Lung Cancer 2004;46:87-98.

9. Fournel P, Robinet G, Thomas $P$, et al. Randomized phase III trial of sequential chemoradiotherapy compared with concurrent chemoradiotherapy in locally advanced non-small-cell lung cancer: Groupe Lyon-Saint-Etienne d'Oncologie Thoracique-Groupe Francais de PneumoCancerologie NPC 95-01 Study. J Clin Oncol 2005;23:5910-5917.

10. Furuse K, Fukuoka M, Kawahara M, et al. Phase III study of concurrent versus sequential thoracic radiotherapy in combination with mitomycin, vindesine, and cisplatin in unresectable stage III non-small-cell lung cancer. J Clin Oncol 1999;17:2692-2699.

11. Belderbos J, Heemsbergen W, Hoogeman M, et al. Acute esophageal toxicity in non-small cell lung cancer patients after high dose conformal radiotherapy. Radiother Oncol 2005;75:157164.

12. Firat S, Byhardt RW, Gore E. Comorbidity and Karnofksy performance score are independent prognostic factors in stage III non-small-cell lung cancer: an institutional analysis of patients treated on four RTOG studies. Radiation Therapy Oncology Group. Int J Radiat Oncol Biol Phys 2002;54:357-364.

13. Martel MK, Ten Haken RK, Hazuka MB, et al. Estimation of tumor control probability model parameters from 3-D dose distributions of non-small cell lung cancer patients. Lung Cancer 1999;24:31-37.

14. Kocak Z, Borst GR, Zeng J, et al. Prospective assessment of dosimetric/physiologic-based models for predicting radiation pneumonitis. Int J Radiat Oncol Biol Phys 2007;67:178-186.

15. Kwa SL, Lebesque JV, Theuws JC, et al. Radiation pneumonitis as a function of mean lung dose: an analysis of pooled data of 540 patients. Int J Radiat Oncol Biol Phys 1998;42:1-9.

16. Wang S, Liao Z, Wei X, et al. Analysis of clinical and dosimetric factors associated with treatment-related pneumonitis (TRP) in patients with non-small-cell lung cancer (NSCLC) treated with concurrent chemotherapy and three-dimensional conformal radiotherapy (3D-CRT). Int $J$ Radiat Oncol Biol Phys 2006;66:1399-1407.

17. Bradley J, Graham MV, Winter K, et al. Toxicity and outcome results of RTOG 9311: a phase III dose-escalation study using three-dimensional conformal radiotherapy in patients with inoperable non-small-cell lung carcinoma. Int J Radiat Oncol Biol Phys 2005;61:318-328. 
18. Kong FM, Hayman JA, Griffith KA, et al. Final toxicity results of a radiation-dose escalation study in patients with non-small-cell lung cancer (NSCLC): predictors for radiation pneumonitis and fibrosis. Int J Radiat Oncol Biol Phys 2006;65:1075-1086.

19. De Ruysscher D, Wanders S, Minken A, et al. Effects of radiotherapy planning with a dedicated combined PET-CT-simulator of patients with non-small cell lung cancer on dose limiting normal tissues and radiation dose-escalation: a planning study. Radiother Oncol 2005;77:5-10.

20. van Der Wel A, Nijsten S, Hochstenbag M, et al. Increased therapeutic ratio by 18FDG-PET CT planning in patients with clinical CT stage N2-N3M0 non-small-cell lung cancer: a modeling study. Int J Radiat Oncol Biol Phys 2005;61:649-655.

21. De Ruysscher D, Wanders S, van Haren E, et al. Selective mediastinal node irradiation based on FDG-PET scan data in patients with non-small-cell lung cancer: a prospective clinical study. Int J Radiat Oncol Biol Phys 2005;62:988-994.

22. Fowler JF, Tome WA, Fenwick JD, et al. A challenge to traditional radiation oncology. Int $J$ Radiat Oncol Biol Phys 2004;60:1241-1256.

23. van Baardwijk A, Bosmans G, Bentzen SM, et al. Radiation dose prescription for non-small cell lung cancer based on normal tissue dose constraints: an in silico trial. Int J Radiat Biol 2008; Feb 5: Epub ahead of print.

24. Calvert AH, Newell DR, Gumbrell LA, et al. Carboplatin dosage: prospective evaluation of a simple formula based on renal function. J Clin Oncol 1989;7:1748-1756.

25. Senan S, De Ruysscher D, Giraud P, et al. Literature-based recommendations for treatment planning and execution in high-dose radiotherapy for lung cancer. Radiother Oncol 2004;71:139-146.

26. ICRU. International Commission on Radiation Units and Measurements Report 50: Prescribing, recording, and reporting photon beam therapy; 1993.

27. Nijsten SM, Mijnheer BJ, Dekker AL, et al. Routine individualised patient dosimetry using electronic portal imaging devices. Radiother Oncol 2007;83:65-75.

28. Schultheiss TE, Kun LE, Ang KK, et al. Radiation response of the central nervous system. Int J Radiat Oncol Biol Phys 1995;31:1093-1112.

29. Kelsey CR, Kahn D, Hollis DR, et al. Radiation-induced narrowing of the tracheobronchial tree: an in-depth analysis. Lung Cancer 2006;52:111-116.

30. Maguire PD, Marks LB, Sibley GS, et al. 73.6 Gy and beyond: hyperfractionated, accelerated radiotherapy for non-small-cell lung cancer. J Clin Oncol 2001;19:705-711.

31. Marks LB, Garst J, Socinski MA, et al. Carboplatin/paclitaxel or carboplatin/vinorelbine followed by accelerated hyperfractionated conformal radiation therapy: report of a prospective phase I dose escalation trial from the Carolina Conformal Therapy Consortium. J Clin Oncol 2004;22:4329-4340.

32. Young $H$, Baum R, Cremerius $U$, et al. Measurement of clinical and subclinical tumour response using (18F)-fluorodeoxyglucose and positron emission tomography: review and 1999 EORTC recommendations. European Organization for Research and Treatment of Cancer (EORTC) PET Study Group. Eur J Cancer 1999;35:1773-1782.

33. Therasse $P$, Arbuck SG, Eisenhauer EA, et al. New guidelines to evaluate the response to treatment in solid tumors. European Organization for Research and Treatment of Cancer, National Cancer Institute of the United States, National Cancer Institute of Canada. J Natl Cancer Inst 2000;92:205-216.

34. Mac Manus MP, Hicks RJ, Matthews JP, et al. Positron emission tomography is superior to computed tomography scanning for response-assessment after radical radiotherapy or chemoradiotherapy in patients with non-small-cell lung cancer. J Clin Oncol 2003;21:1285-1292.

35. van Baardwijk A, Bosmans G, Dekker A, et al. Time trends in the maximal uptake of FDG on PET scan during thoracic radiotherapy. A prospective study in locally advanced non-small cell lung cancer (NSCLC) patients. Radiother Oncol 2007;82:145-152.

36. Saunders $M$, Dische S, Barrett A, et al. Continuous hyperfractionated accelerated radiotherapy (CHART) versus conventional radiotherapy in non-small-cell lung cancer: a randomised multicentre trial. CHART Steering Committee. Lancet 1997;350:161-165.

37. Rodrigues G, Lock M, D'Souza D, et al. Prediction of radiation pneumonitis by dose - volume histogram parameters in lung cancer--a systematic review. Radiother Oncol 2004;71:127-138. 
38. Willner J, Baier K, Caragiani E, et al. Dose, volume, and tumor control prediction in primary radiotherapy of non-small-cell lung cancer. Int J Radiat Oncol Biol Phys 2002;52:382-389.

39. Rengan R, Rosenzweig KE, Venkatraman $\mathrm{E}$, et al. Improved local control with higher doses of radiation in large-volume stage III non-small-cell lung cancer. Int J Radiat Oncol Biol Phys 2004;60:741-747.

40. Curran WJ, Jr. Evolving chemoradiation treatment strategies for locally advanced non-small-cell lung cancer. Oncology (Williston Park) 2003;17:7-14.

41. Groen HJ, van der Leest AH, Fokkema E, et al. Continuously infused carboplatin used as radiosensitizer in locally unresectable non-small-cell lung cancer: a multicenter phase III study. Ann Oncol 2004; 15:427-432.

42. Huber RM, Flentje M, Schmidt M, et al. Simultaneous chemoradiotherapy compared with radiotherapy alone after induction chemotherapy in inoperable stage IIIA or IIIB non-small-cell lung cancer: study CTRT99/97 by the Bronchial Carcinoma Therapy Group. J Clin Oncol $2006 ; 24: 4397-4404$. 


Discussion

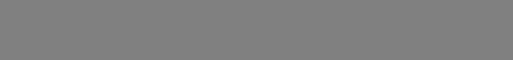





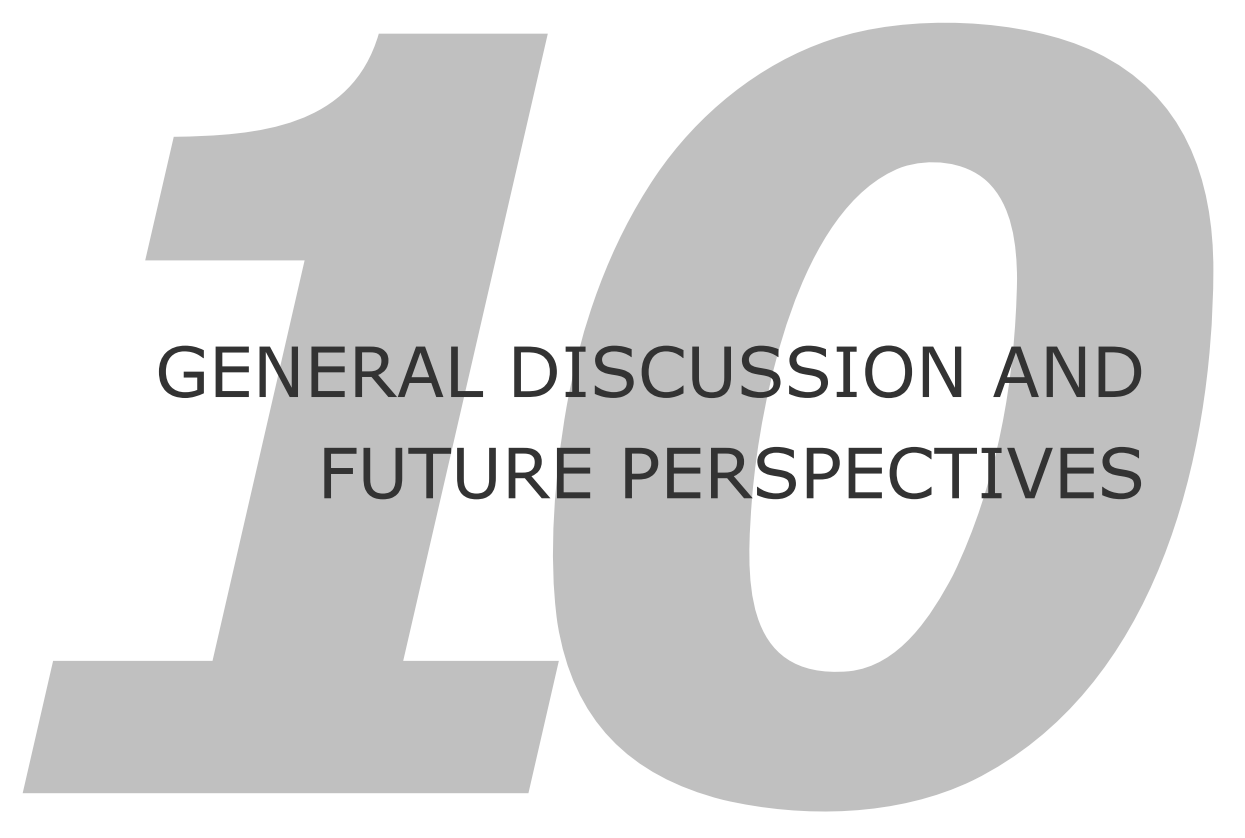





\section{Discussion}

Non-small cell lung cancer (NSCLC) is one of the most frequent cancers diagnosed ${ }^{1,2}$. Many patients are diagnosed in an advanced stage of the disease. Although resection is regarded as the treatment with the highest probability of long-term remission and cure, only about $25 \%$ of NSCLC tumours are suitable for potential curative resection ${ }^{3}$. Lung cancer has in general a poor prognosis, with a 5-year overall survival of about $15 \%{ }^{2,4,5}$. Whereas the treatment of choice for early stage NSCLC is surgery, inoperable and locally advanced disease is mainly treated with radiation alone or radiotherapy combined with chemotherapy. The main problem of radiotherapy in NSCLC is the high risk of local tumour failure. Moreover, a high number of patients develop distant metastases, and therefore it is associated with a poor overall survival. Several issues need to be solved, which might contribute to improvement of radiation treatment outcome in NSCLC. Among these are: a correct delineation of the target volume, the prescription of an adequate total radiation dose to increase tumour control and an adequate delivery of the dose taking into account tumoural changes during radiotherapy. The aim of the work described in this thesis was to optimize radiation treatment in NSCLC, and the role of PET-CT into achieving this goal.

\section{Target volume delineation}

Radiation dose delivery is preceded by treatment planning. Generally a CT scan is used for target volume delineation. The advantage of CT-based planning is that a CT gives detailed information of the anatomy of the patient; it shows the relation between tumour and healthy tissue and allows for the correction of tissue inhomogeneities in radiation treatment planning. An adequate delineation of the target volume is a prerequisite for a precise radiation treatment. If the delineation is not performed correctly, it will influence the total chain of radiation treatment. Moreover, geographical miss of the tumour during radiotherapy will inverse the probability of loco-regional control. First of all, delineation accuracy can be improved using a standardized delineation protocol. Such a protocol should include fixed window-level settings for both the primary tumour and mediastinal lymph nodes.

Nowadays PET(-CT) is increasingly used as pre-treatment scan in radiotherapy ${ }^{6-21}$. PET has a high accuracy for mediastinal staging in NSCLC compared with CT; PET shows a sensitivity of about $80 \%-90 \%$ and a specificity of $70-90 \%$, compared with a sensitivity of CT between $30 \%$ and $80 \%{ }^{22-25}$. Therefore, the use of PET to determine the nodal target volume will improve target volume delineation. Moreover, PET might be used to determine the border between tumour and atelectatic lung tissue. The introduction of PET into target volume delineation has therefore changed the volume and localization of the delineated gross tumour volume (GTV)

In addition the introduction of PET into target volume delineation in lung cancer has consistently shown a reduction in interobserver variation compared with CT-based delineation ${ }^{12,18,26-28}$. In the currently used procedures, target volume delineation is mainly carried out manually. In contrast to CT, PET signals are very suitable for automated segmentation. Several methods have been described to achieve this. The most often used auto-segmentation methods are based on a certain intensity level, e.g. a fixed standardized uptake value (SUV) or a percentage of the maximal SUV ${ }^{11,15,29-34}$. 
SUV is defined as the average activity per unit volume normalized to the injected dose and patient body weight, and is influenced by several factors. Among the factors that interfere with SUV are the volume and shape of the Region Of Interest (ROI), the partial-volume effect, attenuation correction and reconstruction methods ${ }^{35-39}$. SUV is also influenced by the time of evaluation of PET images and by the normalization factor. Therefore, the usage of absolute SUV for auto-segmentation is only reliable in a standardized setting and findings cannot be generally applied due to inter-institutional differences. More sophisticated methods for automated segmentation are the sourceto-background (SBR) method, three segmentation methods-thresholding, Sobel edge detection, the background-subtracted relative-threshold level (RTL) method and the gradient-based watershed approach ${ }^{32,40-43}$. Yet, most of the above mentioned methods are only validated in phantom studies. Therefore, it is not totally clear which is the best method to be used in clinic, and this still needs to be further evaluated. As pathologic examination is the golden standard, ideally these methods need to be validated with histology. Until now, this is only done in laryngeal tumours, for both the SBR method and the gradient-based segmentation method ${ }^{41,44}$. In this thesis the SBR method for NSCLC has been validated and showed a good correlation with pathology for both the primary tumour as well as for the lymph nodal areas. Moreover, PET based automatic delineation will further reduce interobserver variation in target volume delineation (Chapter 5). Auto-contouring can therefore improve daily practice target volume delineation and will facilitate and standardize target volume delineation in multiinstitutional studies.

\section{Margins}

Not only precise target volume delineation but also the margins used for clinical target volume (CTV) and planning target volume (PTV) are of critical importance. The CTV is created by adding a certain margin to the GTV to allow for microscopic extension ${ }^{45}$. These margins are based on the correlation between pathologic examination and image-based delineation. Giraud et al. compared the pre-treatment CT-based diameter of the tumour with the diameter at histological examination in 70 NSCLC patients ${ }^{46}$. They observed that a $5 \mathrm{~mm}$ margin from GTV to CTV will cover about $80 \%$ of microscopic extension in case of adenocarcinoma and $91 \%$ for squamous cell carcinoma. To encompass all microscopic disease in at least $95 \%$ of patients, a margin of $8 \mathrm{~mm}$ for adenocarcinoma and $6 \mathrm{~mm}$ for squamous cell carcinoma was needed. Additional studies investigated the relation between tumour size on CT and histology ${ }^{47,48}$. Grills and colleagues observed in a 35 patient study that CT underestimated tumour size and they found that a CTV margin of $9 \mathrm{~mm}$ was needed to cover microscopic extension for $90 \%$ of cases $^{47}$. In contrast, Chan et al. found in a pilot study of 5 patients that the CT-based GTV overestimated the area of microscopic disease, resulting in no margin needed from GTV to CTV ${ }^{48}$. However, in this study for most patients only bidimensional pathologic measurements were available and no information was given regarding the window-level settings used for CT, which certainly influences CT-based diameters as shown by Grills et al. ${ }^{47}$. Currently a study is performed to gain more insight into the exact position of PET and CT in tumour border definition compared with actual microscopic extension in NSCLC, taking into account the lung deformation due to surgery ${ }^{49}$.

Only limited information is available for the margins needed for lymph nodes. Yuan and colleagues reviewed nodal extracapsular extension in 243 patients and found that the 
extent of extracapsular extension was in $95 \%$ less than $3 \mathrm{~mm}^{50}$. The CTV for lymph nodes to encompass extracapsular node extension should therefore be at least $3 \mathrm{~mm}$.

For radiation treatment planning an additional margin is added to create the PTV. Whereas CTV covers the microscopic extension, the margin used for PTV covers geometric uncertainties as well as general tumour motion ${ }^{51}$. A standard planning CT is a free-breathing $\mathrm{CT}$, which is a snapshot in time and therefore, does not take into account respiratory movement of the tumour. A respiratory correlated CT (RC-CT) is a CT scan during which the respiration signal is recorded and therefore gives information about the tumour motion in all directions during respiration ${ }^{52-54}$. To correct adequately for tumour motion due to respiration, a RC-CT should be used to assess the extent of tumour motion due to respiration, and to calculate an individual adapted margin to compensate for this movement ${ }^{52}$.

\section{Biological meaning of FDG uptake on PET}

It was repeatedly shown that a high $\mathrm{SUV}_{\max }$ is correlated with an inferior overall survival in NSCLC, both in patients treated with surgery as well as radiotherapy ${ }^{55-59}$. The semi-quantitative SUV is a representation of the underlying tumour metabolism, although the exact mechanism by which glycolysis is upregulated within the tumour has not fully been unravelled ${ }^{35}$. Proliferative tumour activity might play a role in the amount of uptake of ${ }^{18} \mathrm{~F}$-fluoro-2-deoxy-glucose (FDG). Veselle et al. and Nguyen et al. showed a correlation between $\mathrm{Ki}-67$ expression and SUV $\max$, although this finding was not observed by others ${ }^{56,60-62}$. Contradictory findings have also been observed, regarding the (over)expression of the glucose transporters and the uptake of FDG in NSCLC $56,60,63-65$. These differences in findings might partially be explained the use of different methods, antibodies, scoring methods and cut-off levels used in the studies. Another candidate, which might play an important role in the FDG uptake, is hypoxia. Stabilization of the Hypoxia Inducible Factor-1a (HIF-1a) pathway under hypoxic conditions will lead to the upregulation of glucose transporters, the vascular endothelial growth factor (VEGF), carbonic anhydrase IX (CAIX) and other target genes ${ }^{66,67}$. Preclinical studies, both in tumour cell lines and in animal models, suggest that hypoxic conditions correspond to a higher FDG uptake $62,68,69$. In a mouse model the uptake of FDG in a Von Hippel Lindau (VHL) knockdown tumour was two-fold increased compared with controls $^{70}$. This might indicate that HIF plays a role in the uptake of FDG, since carcinomas with mutations in the VHL-gene show a high expression of HIF. We confirmed that hypoxia, as expressed by HIF-1a, in vivo is associated with an increase in uptake of FDG (Chapter 3). In addition, hypoxia is known to be an important cause of treatment failure in many tumours. Overexpression of both HIF-1a and CAIX, a pathway regulating $\mathrm{pH}$ within the cell, has been related to a poor outcome $\mathrm{e}^{71-75}$. Therefore, the correlation between a high uptake of FDG with a high expression of the hypoxia-related marker HIF-1 a, might explain the underlying mechanism of the relation between a high uptake of FDG and a poor outcome.

Although the maximal uptake of FDG within a tumour is related to outcome, one has to realize that the uptake of FDG is heterogeneous within the tumour. This variation in uptake of FDG might reflect heterogeneity of ongoing pathophysiological processes. The identification of the intra-tumour heterogeneity for different tumour characteristics by non-invasive imaging techniques, like PET, would allow specific targeting ${ }^{76}$. One of 
the possibilities is radiation surdosage of the areas with a high uptake of FDG. Therefore, it would be of great interest to further investigate the biological background of the intra-tumour heterogeneity in NSCLC for FDG uptake on PET scan, applying the 'ex-vivo' method described in Chapter 4.

\section{Repeated PET-CT scanning: before, during and after radiotherapy}

As described above, the PET(-CT) scan is increasingly used in radiation treatment planning of NSCLC. Although visual interpretation of PET-CT images is still dominating, auto-segmentation can contribute to a more standardized and improved use of PET in target volume delineation ${ }^{6,10-21}$. PET scan would be even of more clinical value, if it would enable prediction of response to radiation treatment early during the course of fractionated radiotherapy. The concept could be similar to early response measurements during chemotherapy in other tumour sites and the monitoring of neo-adjuvant therapy in NSCLC ${ }^{77-80}$. Repeated PET scanning during radiotherapy might allow for early metabolic response prediction, and would give the opportunity to individually adjust ongoing treatment, which might consists of adapting the radiation dose or adding another form of treatment. Moreover, repeated CT scanning should be used to adapt target volume in case of an enlargement of volume during radiation, to avoid geographical misses. This enlargement of volume during fractionated radiotherapy is seen in about $15 \%$ of patients, as described in Chapter 6 . Whether radiation fields should be adapted in case of a decrease in GTV is less clear. No information is available concerning the remaining (microscopic) tumour load left behind in the surrounding tissues, which were originally included in the GTV.

Furthermore, PET can be used to assess treatment outcome after radiotherapy ${ }^{81-85}$. In a study performed by Wong et al. the change in maximal SUV after radiotherapy was more accurate in predicting local control than the change in CT-based tumour size ${ }^{82}$. We confirmed that patients showing a metabolic response on the post-radiotherapy scan showed a better overall survival compared with non-responders (Chapter 7$)^{83}$.

\section{Dose escalation}

Applying radiation biology principles a higher radiation dose should result in a higher local control probability. It is estimated that a total tumour dose (TTD) of 84 Gy (2 Gy fractions, once daily) is needed to achieve about $50 \%$ tumour control probability ${ }^{86}$. Several clinical dose escalation trials have shown promising results ${ }^{87-92}$. Timmerman and colleagues performed a dose-escalation study in patients with medically inoperable, stage I disease using extra-cranial stereotactic radiotherapy ${ }^{88}$. Most other doseescalation studies have been performed in a combined group of patients with stage I to III disease and showed higher local control rates and/or a better overall survival in patients receiving the highest dose ${ }^{91,93}$. Dose escalation is limited by damage to normal tissues. The most critical organs in radiation treatment of NSCLC are the lungs, the spinal cord, but also mediastinal structures, like great vessels and main bronchi. Several dosimetric factors for normal lung tissue, such as relative $V_{20}$ (volumes of lung receiving more than $20 \mathrm{~Gy}$ ) and mean lung dose (MLD) have been shown to be useful to estimate the risk of radiation pneumonitis ${ }^{95,96}$. The use of (FDG) PET-CT scans for target volume delineation in NSCLC might further allow dose escalation, since its use decreased radiation exposure of the oesophagus and lung ${ }^{6,17}$. This decrease in 
exposure of normal tissues indeed allowed for a significant radiation dose escalation, which lead to an increase in estimated tumour control probability ${ }^{6,17}$. Omitting elective irradiation of the mediastinum, and selectively irradiate pathologic lymphnodal areas, also allows for dose-escalation. At least 2 prospective studies have shown that it is safe to selectively irradiate the FDG-PET positive mediastinal nodes ${ }^{91,100}$.

However, not only the TTD delivered, but also the overall treatment time in which the radiation treatment is given is an important determinant of outcome. In the CHART trial a hyperfractionated, accelerated scheme ( $54 \mathrm{~Gy}$ in 12 days, $1.5 \mathrm{~Gy} 3$ times per day) was compared with a conventional scheme of $60 \mathrm{~Gy}$ in 6 weeks, $2.0 \mathrm{~Gy}$ once daily ${ }^{101}$. This trial showed a 2 -year overall survival of $30 \%$ for the CHART schedule compared with $21 \%$ for the conventional scheme. Moreover, the recent CHARTWELL study confirmed the importance of overall treatment time ${ }^{102}$.

Most of the above mentioned dose escalation studies have used strict inclusion criteria, e.g. invasion in the great vessels and/or involved supraclavicular nodes were not allowed $^{91,93}$. Therefore, the results of dose escalation and accelerated schedules can only be applied to this selected subpopulation of NSCLC patients. Especially in case of a small tumour volume the TTD can be easily escalated up to higher doses, whereas the possibilities for larger tumour volumes are limited. Especially the more difficult cases, with tumours growing directly into the mediastinum or great vessels or tumours located in the direct proximity of the spinal cord are challenging. It is also important in these cases to achieve the best result possible for each individual patient. We therefore applied an individualized radiation schedule including the highest achievable TTD based on individualized normal tissue dose constraints and delivered in a short overall treatment time. A theoretical gain in total tumour control probability of $25 \%$ can probably be achieved using a personalized maximal tolerable dose scheme, given in twice daily fractions of $1.8 \mathrm{~Gy}$ instead of a classic scheme of $60 \mathrm{~Gy}$ in $2.0 \mathrm{~Gy}$ fractions once daily. The results of our in silico trial were confirmed in our prospective clinical trial (Chapter 8 and 9).

\section{General Conclusions}

Due to its high diagnostic accuracy in NSCLC, PET-CT scans have acquired their place in radiation treatment of NSCLC. The uptake of FDG on PET scan has also a prognostic value, since a high uptake is correlated with a poor survival. The amount of uptake of FDG as seen on pre-treatment scans is associated with the expression of hypoxia

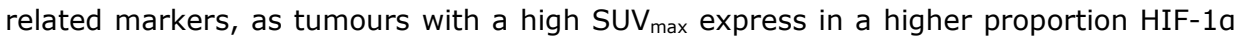
and GLUT-1 compared with tumours with a low uptake.

PET-CT scans are increasingly used for target volume delineation. Furthermore, PET can be used for automatic segmentation. The source-to-background (SBR) automatic segmentation method has been validated in NSCLC and not only reduces the delineated volume of the GTV, but also reduces interobserver variation in target volume delineation. Therefore, auto-contouring should be used to standardize target volume delineation in multi-institutional studies and can be easily applied in daily practice. 
PET-CT scans should however not only be used before start of radiation therapy, but repeated scanning during therapy is needed. This will allow adjustment of radiation fields according to changes in target volumes during radiotherapy, to avoid geographical misses. The repeated scans also provide information regarding the evolution of FDG uptake during fractionated radiotherapy, which may be prognostic for outcome.

Finally, the use of FDG-PET scans for target volume delineation shows smaller target volumes. This leads to decreased radiation exposure of normal tissues and will therefore allow for dose-escalation. Applying an individualized dose prescription, delivering the maximal tolerable dose based on individual dose constraints, will ultimately lead to more favourable results of radiation treatment in NSCLC.

\section{Future perspectives}

Although the use of PET next to CT improves target volume delineation in NSCLC, several issues implementing $\mathrm{PET}(-\mathrm{CT})$ in radiation treatment remain unresolved. Some of these are related to respiratory tumour motion. In contrast to the fast acquisition of $\mathrm{CT}$, the acquisition of a standard PET image takes 3 to 5 minutes. If a PET is performed during free breathing, it consists of an average image of multiple respiratory cycles. Therefore, it blurs the target volume and might result in an overestimation of the GTV. Methods to correct for this respiratory motion, such as respiratory-correlated PET (RC-PET) are developed to overcome this problem. The combined use of a respiration correlated CT and PET scan might further improve the estimation of the tumour volume and the exact localization of the tumour ${ }^{103,104}$. Moreover, applying RC-PET will also affect the measured level of SUV and can influence maximal uptake values in up to $30 \%$ compared with static images ${ }^{38,103}$. If both respiration correlation and phased attenuation correction are applied, the determination of maximal SUV will be more reliable and thus better suited for tumour characterization and might also further improve PET based auto-segmentation. The assessment of individual tumour movement will also enable individualisation of the margins used for PTV, and thus reduce the risk of geographical miss. In case of limited tumour movement, individual margins for PTV might be condensed, allowing for further dose escalation.

Other issues that need to be further addressed are the spatial resolution of PET and tumour edge definition. The current spatial resolution of PET ( 5 to $6 \mathrm{~mm}$ ) is large compared with the spatial resolution of CT (about $1 \mathrm{~mm}$ ). The limited spatial resolution of PET contributes to the blurring of the image and might therefore also influence the tumour edge definition. Although technical advances might improve the resolution of PET, it is likely to be limited to a resolution of 2 to $3 \mathrm{~mm}$.

${ }^{18} \mathrm{~F}$-FDG is the most widely available and used tracer for PET in oncology. Although the uptake of FDG is correlated with outcome, FDG is not a very specific tracer. FDG is likely to be related to clonogenic density and might be seen as general tracer for the identification of tumour cells within the body. False-positive findings can be caused by inflammation or physiological uptake, whereas false-negative scans can occur in slowly growing tumours. However, more specific tracers are currently being evaluated ${ }^{49,105}$. One field of interest is the imaging of hypoxia. ${ }^{18} \mathrm{~F}$-labeled Fluoromisonidazole 
$\left({ }^{18} \mathrm{~F}\right.$-MISO $)$ is the most widely examined nitroimidazole derivative, representing a noninvasive method for the quantification of the oxygenation status. Although F-MISO is feasible for imaging, the clinical use is limited due to a low tumour-to-background ratio and the slow clearance from normoxic cells ${ }^{49,106}$. A more promising PET tracer for the visualization of tumour hypoxia might be the ${ }^{18} \mathrm{~F}$-labeled nitroimidazole compound fluoroazomycin arabinoside $\left({ }^{18} \mathrm{~F}-\mathrm{FAZA}\right)$, since this tracer has a higher tumour-tobackground ratio compared with ${ }^{18} \mathrm{~F}-\mathrm{MISO}^{107,108}$. The hypoxia related tracers might also be useful in predicting response to treatment ${ }^{85,109}$. Moreover, specific tracers to image cellular proliferation and apoptosis are investigated. Another interesting field of research concerns the coupling of monoclonal antibodies to radioisotopes for $\mathrm{PET}^{110,111}$. This will enable the evaluation of the binding properties of the monoclonal antibodies and the visualization of the presence of receptors for these antibodies in vivo. Labeling of the Epidermal Growth Factor Receptor (EGFR) inhibitor Cetuximab to the positron emitter ${ }^{89}$ Zirconium, might give the opportunity to visualize the binding of Cetuximab in vivo and can therefore be used to specific target EGFR ${ }^{110}$. A similar approach might be applied to image hypoxia in vivo and to target CAIX ${ }^{112}$.

One has to be aware that tumours are not homogenous, but actually heterogeneous for several biological characteristics ${ }^{113}$. It is the ultimate aim to visualize these tumour characteristics by imaging ${ }^{76}$. Potentially PET might be used to map these different areas within the tumour with specific characteristics. Selective targeting of the most radioresistant areas within a tumour might improve local control rates and therefore survival in the radiation treatment of NSCLC. One of the most essential questions to be addressed at the outset is whether areas with a high uptake of FDG remain stable during fractionated radiotherapy. Moreover, one has to get informed about the exact localization of residual disease and/or recurrence after radiotherapy. If recurrences are predominantly detected in the pre-treatment FDG hotspots and moreover, if these areas remain stable during fractionated radiotherapy, this would allow selective subboosting of these specific areas. Therefore, we are currently investigating the stability of FDG uptake during radiotherapy and the localisation of residual disease ${ }^{114}$. One of the problems which need to be tackled is the comparison and matching of post- and pre-radiation scans correctly, since deformation is encountered for both normal tissues as well as tumour after radiation treatment.

In conclusion, (radiation) treatment for NSCLC needs to be further individualized. First of all, the treatment modality of choice for an individual patient should not only be based on TNM-classification and the general condition of the patient. More dedicated tools are needed to predict outcome. A method to achieve this is the use of the socalled computer assisted theranostics. The term theranostics refers to diagnostic tests that identify the suitability of a specific treatment for an individual patient. Treatment can be individually utilized according to test results. This concept is usually referring to targeted drugs, but might also be applicable to radiation treatment. Tools are needed to select individual patients for combined treatments, like chemo-radiation or a combination of radiotherapy and monoclonal antibodies and/or new molecules. The use of new tracers and labelled antibodies in molecular imaging might assist in this selection procedure. 
Moreover, in case of radiotherapy the target volume and margins used need to be further individualized. Improvement in auto-delineation methods, combined with the use of more dedicated imaging, including RC-PET-CT will indeed improve target volume delineation and will allow determination of individual margins.

Once treatment has started, therapy needs to be adapted according to treatment induced changes. For radiotherapy this means that radiation treatment planning should not only be based on pre-treatment scans, but individual changes in tumour volume, tumour movement and even metabolic changes during therapy should be taken into account. The shape and size of radiation fields, the total tumour dose, as well as the dose distribution within the tumour and even the combination of several treatment modalities need to be reconsidered during therapy. Treatment needs to be adapted according to the individual needs of both the patient, as well as the tumour. Further individualization will ultimately lead to an improvement of (radiation) treatment in NSCLC. 


\section{References}

1. Ferlay J, Autier $\mathrm{P}$, Boniol $\mathrm{M}$, et al. Estimates of the cancer incidence and mortality in Europe in 2006. Ann Oncol 2007;18:581-592.

2. Jemal A, Siegel R, Ward E, et al. Cancer statistics, 2007. CA Cancer J Clin 2007;57:43-66.

3. van Zandwijk N. Neoadjuvant strategies for non-small cell lung cancer. Lung Cancer 2001;34 Suppl 2:S145-150.

4. Damhuis RAM, van Dijck JAAM, Siesling S, et al. Lung cancer and mesothelioma in the Netherlands 1989-1997. In: Vereniging van Integrale Kankercentra; 2000. pp. 20-23.

5. Sant M, Aareleid T, Berrino F, et al. EUROCARE-3: survival of cancer patients diagnosed 199094--results and commentary. Ann Oncol 2003;14 Suppl 5:v61-118.

6. De Ruysscher $D$, Wanders $S$, Minken $A$, et al. Effects of radiotherapy planning with a dedicated combined PET-CT-simulator of patients with non-small cell lung cancer on dose limiting normal tissues and radiation dose-escalation: a planning study. Radiother Oncol 2005;77:5-10.

7. Mac Manus MP, Hicks RJ. PET scanning in lung cancer: current status and future directions. Semin Surg Oncol 2003;21:149-155.

8. Macmanus M, D'Costa I, Everitt S, et al. Comparison of CT and positron emission tomography/CT coregistered images in planning radical radiotherapy in patients with non-small-cell lung cancer. Australas Radiol 2007;51:386-393.

9. Munley MT, Marks LB, Scarfone C, et al. Multimodality nuclear medicine imaging in threedimensional radiation treatment planning for lung cancer: challenges and prospects. Lung Cancer 1999;23:105-114.

10. Grills IS, Yan D, Black QC, et al. Clinical implications of defining the gross tumor volume with combination of CT and 18FDG-positron emission tomography in non-small-cell lung cancer. Int J Radiat Oncol Biol Phys 2007;67:709-719.

11. Black QC, Grills IS, Kestin LL, et al. Defining a radiotherapy target with positron emission tomography. Int J Radiat Oncol Biol Phys 2004;60:1272-1282.

12. Ciernik IF, Dizendorf $E$, Baumert BG, et al. Radiation treatment planning with an integrated positron emission and computer tomography (PET/CT): a feasibility study. Int J Radiat Oncol Biol Phys 2003; 57:853-863.

13. Deniaud-Alexandre E, Touboul E, Lerouge D, et al. Impact of computed tomography and 18Fdeoxyglucose coincidence detection emission tomography image fusion for optimization of conformal radiotherapy in non-small-cell lung cancer. Int J Radiat Oncol Biol Phys 2005;63:14321441.

14. Kiffer JD, Berlangieri SU, Scott AM, et al. The contribution of 18F-fluoro-2-deoxy-glucose positron emission tomographic imaging to radiotherapy planning in lung cancer. Lung Cancer 1998;19:167-177.

15. Mah K, Caldwell CB, Ung YC, et al. The impact of (18)FDG-PET on target and critical organs in CT-based treatment planning of patients with poorly defined non-small-cell lung carcinoma: a prospective study. Int J Radiat Oncol Biol Phys 2002;52:339-350.

16. Nestle U, Walter K, Schmidt S, et al. 18F-deoxyglucose positron emission tomography (FDGPET) for the planning of radiotherapy in lung cancer: high impact in patients with atelectasis. Int J Radiat Oncol Biol Phys 1999;44:593-597.

17. van Der Wel $A$, Nijsten $S$, Hochstenbag $M$, et al. Increased therapeutic ratio by $18 F D G-P E T$ CT planning in patients with clinical CT stage N2-N3MO non-small-cell lung cancer: a modeling study. Int J Radiat Oncol Biol Phys 2005;61:649-655.

18. Ashamalla $\mathrm{H}$, Rafla $\mathrm{S}$, Parikh $\mathrm{K}$, et al. The contribution of integrated PET/CT to the evolving definition of treatment volumes in radiation treatment planning in lung cancer. Int J Radiat Oncol Biol Phys 2005;63:1016-1023.

19. Bradley J, Thorstad WL, Mutic S, et al. Impact of FDG-PET on radiation therapy volume delineation in non-small-cell lung cancer. Int J Radiat Oncol Biol Phys 2004;59:78-86.

20. Erdi $Y E$, Rosenzweig $K$, Erdi $A K$, et al. Radiotherapy treatment planning for patients with nonsmall cell lung cancer using positron emission tomography (PET). Radiother Oncol 2002;62:5160. 
21. Vanuytsel LJ, Vansteenkiste JF, Stroobants SG, et al. The impact of (18)F-fluoro-2-deoxy-Dglucose positron emission tomography (FDG-PET) lymph node staging on the radiation treatment volumes in patients with non-small cell lung cancer. Radiother Oncol 2000;55:317-324.

22. Dwamena BA, Sonnad SS, Angobaldo JO, et al. Metastases from non-small cell lung cancer: mediastinal staging in the 1990s--meta-analytic comparison of PET and CT. Radiology 1999;213:530-536.

23. Fischer BM, Mortensen J, Hojgaard L. Positron emission tomography in the diagnosis and staging of lung cancer: a systematic, quantitative review. Lancet Oncol 2001;2:659-666.

24. Kramer H, Groen $\mathrm{HJ}$. Current concepts in the mediastinal lymph node staging of nonsmall cell lung cancer. Ann Surg 2003;238:180-188.

25. Gould MK, Kuschner WG, Rydzak CE, et al. Test performance of positron emission tomography and computed tomography for mediastinal staging in patients with non-small-cell lung cancer: a meta-analysis. Ann Intern Med 2003;139:879-892.

26. Caldwell CB, Mah K, Ung YC, et al. Observer variation in contouring gross tumor volume in patients with poorly defined non-small-cell lung tumors on CT: the impact of 18FDG-hybrid PET fusion. Int J Radiat Oncol Biol Phys 2001;51:923-931.

27. Fox JL, Rengan R, O'Meara W, et al. Does registration of PET and planning CT images decrease interobserver and intraobserver variation in delineating tumor volumes for non-small-cell lung cancer? Int J Radiat Oncol Biol Phys 2005;62:70-75.

28. Steenbakkers RJ, Duppen JC, Fitton I, et al. Reduction of observer variation using matched CTPET for lung cancer delineation: a three-dimensional analysis. Int $J$ Radiat Oncol Biol Phys 2006;64:435-448.

29. Biehl KJ, Kong FM, Dehdashti F, et al. 18F-FDG PET definition of gross tumor volume for radiotherapy of non-small cell lung cancer: is a single standardized uptake value threshold approach appropriate? J Nucl Med 2006;47:1808-1812.

30. Bradley JD, Dehdashti F, Mintun MA, et al. Positron emission tomography in limited-stage small-cell lung cancer: a prospective study. J Clin Oncol 2004;22:3248-3254.

31. Ciernik IF, Huser M, Burger $C$, et al. Automated functional image-guided radiation treatment planning for rectal cancer. Int J Radiat Oncol Biol Phys 2005;62:893-900.

32. Daisne JF, Sibomana M, Bol A, et al. Tri-dimensional automatic segmentation of PET volumes based on measured source-to-background ratios: influence of reconstruction algorithms. Radiother Oncol 2003;69:247-250.

33. Erdi YE, Mawlawi O, Larson SM, et al. Segmentation of lung lesion volume by adaptive positron emission tomography image thresholding. Cancer 1997;80:2505-2509.

34. Paulino AC, Johnstone PA. FDG-PET in radiotherapy treatment planning: Pandora's box? Int $J$ Radiat Oncol Biol Phys 2004;59:4-5.

35. Thie JA. Understanding the standardized uptake value, its methods, and implications for usage. J Nucl Med 2004;45:1431-1434.

36. Huang SC. Anatomy of SUV. Standardized uptake value. Nucl Med Biol 2000;27:643-646.

37. Boellaard R, Krak NC, Hoekstra OS, et al. Effects of noise, image resolution, and ROI definition on the accuracy of standard uptake values: a simulation study. J Nucl Med 2004;45:1519-1527.

38. Erdi YE, Nehmeh SA, Pan T, et al. The CT motion quantitation of lung lesions and its impact on PET-measured SUVs. J Nucl Med 2004;45:1287-1292.

39. Hoekstra CJ, Paglianiti I, Hoekstra OS, et al. Monitoring response to therapy in cancer using (18F)-2-fluoro-2-deoxy-D-glucose and positron emission tomography: an overview of different analytical methods. Eur J Nucl Med 2000;27:731-743.

40. van Dalen JA, Hoffmann AL, Dicken V, et al. A novel iterative method for lesion delineation and volumetric quantification with FDG PET. Nucl Med Commun 2007;28:485-493.

41. Geets X, Lee JA, Bol A, et al. A gradient-based method for segmenting FDG-PET images: methodology and validation. Eur J Nucl Med Mol Imaging 2007.

42. Drever L, Roa W, McEwan A, et al. Iterative threshold segmentation for PET target volume delineation. Med Phys 2007;34:1253-1265.

43. Drever LA, Roa W, McEwan A, et al. Comparison of three image segmentation techniques for target volume delineation in positron emission tomography. J Appl Clin Med Phys 2007;8:93109. 
44. Daisne JF, Duprez T, Weynand B, et al. Tumor volume in pharyngolaryngeal squamous cell carcinoma: comparison at CT, MR imaging, and FDG PET and validation with surgical specimen. Radiology 2004;233:93-100.

45. ICRU. International Commission on Radiation Units and Measurements Report 50: Prescribing, recording, and reporting photon beam therapy; 1993.

46. Giraud $P$, Antoine M, Larrouy $A$, et al. Evaluation of microscopic tumor extension in non-smallcell lung cancer for three-dimensional conformal radiotherapy planning. Int J Radiat Oncol Biol Phys 2000;48:1015-1024.

47. Grills IS, Fitch DL, Goldstein NS, et al. Clinicopathologic Analysis of Microscopic Extension in Lung Adenocarcinoma: Defining Clinical Target Volume for Radiotherapy. Int J Radiat Oncol Biol Phys 2007.

48. Chan R, He Y, Haque A, et al. Computed tomographic-pathologic correlation of gross tumor volume and clinical target volume in non-small cell lung cancer: a pilot experience. Arch Pathol Lab Med 2001;125:1469-1472.

49. Stroom J, Blaauwgeers $H$, van Baardwijk A, et al. Feasibility of pathology-correlated lung imaging for accurate target definition of lung tumors. Int J Radiat Oncol Biol Phys 2007;69:267275.

50. Yuan S, Meng X, Yu J, et al. Determining optimal clinical target volume margins on the basis of microscopic extracapsular extension of metastatic nodes in patients with non-small-cell lung cancer. Int J Radiat Oncol Biol Phys 2007;67:727-734.

51. van Herk M. Errors and margins in radiotherapy. Semin Radiat Oncol 2004;14:52-64.

52. Bosmans G, Buijsen J, Dekker A, et al. An "in silico" clinical trial comparing free breathing, slow and respiration correlated computed tomography in lung cancer patients. Radiother Oncol 2006;81:73-80.

53. Ford EC, Mageras GS, Yorke E, et al. Respiration-correlated spiral CT: a method of measuring respiratory-induced anatomic motion for radiation treatment planning. Med Phys 2003;30:8897.

54. Mageras GS, Pevsner A, Yorke ED, et al. Measurement of lung tumor motion using respirationcorrelated CT. Int J Radiat Oncol Biol Phys 2004;60:933-941.

55. Borst GR, Belderbos JS, Boellaard R, et al. Standardised FDG uptake: a prognostic factor for inoperable non-small cell lung cancer. Eur J Cancer 2005;41:1533-1541.

56. Nguyen XC, Lee WW, Chung JH, et al. FDG uptake, glucose transporter type 1, and Ki-67 expressions in non-small-cell lung cancer: correlations and prognostic values. Eur $\mathrm{J}$ Radiol 2007;62:214-219.

57. Downey RJ, Akhurst T, Gonen M, et al. Preoperative F-18 fluorodeoxyglucose-positron emission tomography maximal standardized uptake value predicts survival after lung cancer resection. $J$ Clin Oncol 2004;22:3255-3260.

58. Eschmann SM, Friedel G, Paulsen F, et al. Is standardised (18)F-FDG uptake value an outcome predictor in patients with stage III non-small cell lung cancer? Eur J Nucl Med Mol Imaging 2005.

59. Vansteenkiste JF, Stroobants SG, Dupont PJ, et al. Prognostic importance of the standardized uptake value on (18)F-fluoro-2-deoxy-glucose-positron emission tomography scan in nonsmall-cell lung cancer: An analysis of 125 cases. Leuven Lung Cancer Group. J Clin Oncol 1999;17:3201-3206.

60. Chung JK, Lee YJ, Kim SK, et al. Comparison of (18F)fluorodeoxyglucose uptake with glucose transporter-1 expression and proliferation rate in human glioma and non-small-cell lung cancer. Nucl Med Commun 2004;25:11-17.

61. Vesselle H, Schmidt RA, Pugsley JM, et al. Lung cancer proliferation correlates with (F18)fluorodeoxyglucose uptake by positron emission tomography. Clin Cancer Res 2000;6:38373844.

62. Pugachev A, Ruan S, Carlin S, et al. Dependence of FDG uptake on tumor microenvironment. Int J Radiat Oncol Biol Phys 2005;62:545-553.

63. Higashi K, Ueda Y, Sakurai A, et al. Correlation of Glut-1 glucose transporter expression with (18F) FDG uptake in non-small cell lung cancer. Eur J Nucl Med 2000;27:1778-1785. 
64. Mamede M, Higashi T, Kitaichi M, et al. (18F)FDG uptake and PCNA, Glut-1, and Hexokinase-II expressions in cancers and inflammatory lesions of the lung. Neoplasia 2005;7:369-379.

65. Marom EM, Aloia TA, Moore MB, et al. Correlation of FDG-PET imaging with Glut-1 and Glut-3 expression in early-stage non-small cell lung cancer. Lung Cancer 2001;33:99-107.

66. Vaupel P. The role of hypoxia-induced factors in tumor progression. Oncologist 2004;9 Suppl 5:10-17.

67. Zhang JZ, Behrooz A, Ismail-Beigi F. Regulation of glucose transport by hypoxia. Am J Kidney Dis 1999;34:189-202.

68. Burgman P, Odonoghue JA, Humm JL, et al. Hypoxia-Induced increase in FDG uptake in MCF7 cells. J Nucl Med 2001;42:170-175.

69. Minn $\mathrm{H}$, Clavo AC, Wahl RL. Influence of hypoxia on tracer accumulation in squamous-cell carcinoma: in vitro evaluation for PET imaging. Nucl Med Biol 1996;23:941-946.

70. Thomas GV, Tran C, Mellinghoff IK, et al. Hypoxia-inducible factor determines sensitivity to inhibitors of mTOR in kidney cancer. Nat Med 2006;12:122-127.

71. Giatromanolaki A, Koukourakis MI, Sivridis E, et al. Expression of hypoxia-inducible carbonic anhydrase-9 relates to angiogenic pathways and independently to poor outcome in non-small cell lung cancer. Cancer Res 2001;61:7992-7998.

72. Koukourakis MI, Bentzen SM, Giatromanolaki A, et al. Endogenous Markers of Two Separate Hypoxia Response Pathways (hypoxia inducible factor 2 alpha and carbonic anhydrase 9) Are Associated With Radiotherapy Failure in Head and Neck Cancer Patients Recruited in the CHART Randomized Trial. J Clin Oncol 2006.

73. Giatromanolaki A, Koukourakis MI, Sivridis E, et al. Relation of hypoxia inducible factor 1 alpha and 2 alpha in operable non-small cell lung cancer to angiogenic/molecular profile of tumours and survival. Br J Cancer 2001;85:881-890.

74. Kim SJ, Rabbani ZN, Vollmer RT, et al. Carbonic anhydrase IX in early-stage non-small cell lung cancer. Clin Cancer Res 2004;10:7925-7933.

75. Swinson DE, Jones JL, Richardson $\mathrm{D}$, et al. Carbonic anhydrase IX expression, a novel surrogate marker of tumor hypoxia, is associated with a poor prognosis in non-small-cell lung cancer. $J$ Clin Oncol 2003;21:473-482.

76. Bentzen SM. Theragnostic imaging for radiation oncology: dose-painting by numbers. Lancet Oncol 2005;6:112-117.

77. Dose Schwarz J, Bader M, Jenicke L, et al. Early prediction of response to chemotherapy in metastatic breast cancer using sequential 18F-FDG PET. J Nucl Med 2005;46:1144-1150.

78. Eschmann SM, Friedel G, Paulsen F, et al. Repeat 18F-FDG PET for monitoring neoadjuvant chemotherapy in patients with stage III non-small cell lung cancer. Lung Cancer 2007;55:165171.

79. Gallamini A, Rigacci L, Merli F, et al. The predictive value of positron emission tomography scanning performed after two courses of standard therapy on treatment outcome in advanced stage Hodgkin's disease. Haematologica 2006;91:475-481.

80. Rahmouni A, Luciani A, Itti E. MRI and PET in monitoring response in lymphoma. Cancer Imaging 2005;5 Spec No A:S106-112.

81. Mac Manus MP, Hicks RJ, Matthews JP, et al. Positron emission tomography is superior to computed tomography scanning for response-assessment after radical radiotherapy or chemoradiotherapy in patients with non-small-cell lung cancer. J Clin Oncol 2003;21:1285-1292.

82. Wong CY, Schmidt J, Bong JS, et al. Correlating metabolic and anatomic responses of primary lung cancers to radiotherapy by combined F-18 FDG PET-CT imaging. Radiat Oncol 2007;2:18.

83. Mac Manus MP, Hicks RJ, Matthews JP, et al. Metabolic (FDG-PET) response after radical radiotherapy/chemoradiotherapy for non-small cell lung cancer correlates with patterns of failure. Lung Cancer 2005;49:95-108.

84. Pottgen C, Levegrun S, Theegarten D, et al. Value of 18F-fluoro-2-deoxy-D-glucose-positron emission tomography/computed tomography in non-small-cell lung cancer for prediction of pathologic response and times to relapse after neoadjuvant chemoradiotherapy. Clin Cancer Res 2006;12:97-106. 
85. Gagel B, Reinartz P, Demirel C, et al. (18F) fluoromisonidazole and (18F) fluorodeoxyglucose positron emission tomography in response evaluation after chemo-/radiotherapy of non-smallcell lung cancer: a feasibility study. BMC Cancer 2006;6:51.

86. Martel MK, Ten Haken RK, Hazuka MB, et al. Estimation of tumor control probability model parameters from 3-D dose distributions of non-small cell lung cancer patients. Lung Cancer $1999 ; 24: 31-37$.

87. Perez CA, Pajak TF, Rubin P, et al. Long-term observations of the patterns of failure in patients with unresectable non-oat cell carcinoma of the lung treated with definitive radiotherapy. Report by the Radiation Therapy Oncology Group. Cancer 1987;59:1874-1881.

88. Timmerman R, Papiez L, McGarry R, et al. Extracranial stereotactic radioablation: results of a phase I study in medically inoperable stage I non-small cell lung cancer. Chest 2003;124:1946-1955.

89. Marks LB, Garst J, Socinski MA, et al. Carboplatin/paclitaxel or carboplatin/vinorelbine followed by accelerated hyperfractionated conformal radiation therapy: report of a prospective phase I dose escalation trial from the Carolina Conformal Therapy Consortium. J Clin Oncol $2004 ; 22: 4329-4340$.

90. Bradley J. A review of radiation dose escalation trials for non-small cell lung cancer within the Radiation Therapy Oncology Group. Semin Oncol 2005;32:S111-113.

91. Belderbos JS, Heemsbergen WD, De Jaeger K, et al. Final results of a Phase I/II dose escalation trial in non-small-cell lung cancer using three-dimensional conformal radiotherapy. Int $J$ Radiat Oncol Biol Phys 2006;66:126-134.

92. Chen M, Hayman JA, Ten Haken RK, et al. Long-term results of high-dose conformal radiotherapy for patients with medically inoperable T1-3N0 non-small-cell lung cancer: is low incidence of regional failure due to incidental nodal irradiation? Int J Radiat Oncol Biol Phys 2005.

93. Kong FM, Ten Haken RK, Schipper MJ, et al. High-dose radiation improved local tumor control and overall survival in patients with inoperable/unresectable non-small-cell lung cancer: Longterm results of a radiation dose escalation study. Int J Radiat Oncol Biol Phys 2005;63:324333.

94. Belderbos J, Heemsbergen W, Hoogeman M, et al. Acute esophageal toxicity in non-small cell lung cancer patients after high dose conformal radiotherapy. Radiother Oncol 2005;75:157164.

95. Kwa SL, Lebesque JV, Theuws JC, et al. Radiation pneumonitis as a function of mean lung dose: an analysis of pooled data of 540 patients. Int J Radiat Oncol Biol Phys 1998;42:1-9.

96. Wang S, Liao Z, Wei X, et al. Analysis of clinical and dosimetric factors associated with treatment-related pneumonitis (TRP) in patients with non-small-cell lung cancer (NSCLC) treated with concurrent chemotherapy and three-dimensional conformal radiotherapy (3DCRT). Int J Radiat Oncol Biol Phys 2006;66:1399-1407.

97. Rosenzweig KE, Fox JL, Yorke E, et al. Results of a phase I dose-escalation study using threedimensional conformal radiotherapy in the treatment of inoperable nonsmall cell lung carcinoma. Cancer 2005;103:2118-2127.

98. Kong FM, Hayman JA, Griffith KA, et al. Final toxicity results of a radiation-dose escalation study in patients with non-small-cell lung cancer (NSCLC): predictors for radiation pneumonitis and fibrosis. Int J Radiat Oncol Biol Phys 2006;65:1075-1086.

99. Bradley J, Graham MV, Winter K, et al. Toxicity and outcome results of RTOG 9311: a phase III dose-escalation study using three-dimensional conformal radiotherapy in patients with inoperable non-small-cell lung carcinoma. Int J Radiat Oncol Biol Phys 2005;61:318-328.

100. De Ruysscher D, Wanders S, van Haren E, et al. Selective mediastinal node irradiation based on FDG-PET scan data in patients with non-small-cell lung cancer: a prospective clinical study. Int J Radiat Oncol Biol Phys 2005;62:988-994.

101. Saunders M, Dische S, Barrett A, et al. Continuous, hyperfractionated, accelerated radiotherapy (CHART) versus conventional radiotherapy in non-small cell lung cancer: mature data from the randomised multicentre trial. CHART Steering committee. Radiother Oncol 1999;52:137-148. 
102. Baumann M, Herrmann T, Koch R, et al. Continuous hyperfractionated accelerated radiotherapy - weekend less (CHARTWEL) versus conventionally fractionated (CF) radiotherapy in nonsmall-cell lung cancer (NSCLC): first results of a phase III randomised multicentre trial (ARO 971) ECCO 13, 2005. Eur J Cancer 2005;Supplements Vol 3:322.

103. Nagel CC, Bosmans G, Dekker AL, et al. Phased attenuation correction in respiration correlated computed tomography/positron emitted tomography. Med Phys 2006;33:1840-1847.

104. Nehmeh SA, Erdi YE, Pan T, et al. Quantitation of respiratory motion during 4D-PET/CT acquisition. Med Phys 2004;31:1333-1338.

105. Wood KA, Hoskin PJ, Saunders MI. Positron emission tomography in oncology: a review. Clin Oncol (R Coll Radiol) 2007;19:237-255.

106. Apisarnthanarax S, Chao KS. Current imaging paradigms in radiation oncology. Radiat Res 2005;163:1-25.

107. Piert M, Machulla HJ, Picchio M, et al. Hypoxia-specific tumor imaging with $18 \mathrm{~F}$-fluoroazomycin arabinoside. J Nucl Med 2005;46:106-113.

108. Souvatzoglou M, Grosu AL, Roper B, et al. Tumour hypoxia imaging with ((18)F)FAZA PET in head and neck cancer patients: a pilot study. Eur J Nucl Med Mol Imaging 2007.

109. Eschmann SM, Paulsen F, Reimold M, et al. Prognostic impact of hypoxia imaging with $18 \mathrm{~F}-$ misonidazole PET in non-small cell lung cancer and head and neck cancer before radiotherapy. J Nucl Med 2005;46:253-260.

110. Aerts HJ, Dubois L, Hackeng TM, et al. Development and evaluation of a cetuximab-based imaging probe to target EGFR and EGFRvIII. Radiother Oncol 2007;83:326-332.

111. Cai W, Chen K, He L, et al. Quantitative PET of EGFR expression in xenograft-bearing mice using (64)Cu-labeled cetuximab, a chimeric anti-EGFR monoclonal antibody. Eur J Nucl Med Mol Imaging 2007;34:850-858.

112. Dubois L, Douma K, Supuran CT, et al. Imaging the hypoxia surrogate marker CA IX requires expression and catalytic activity for binding fluorescent sulfonamide inhibitors. Radiother Oncol 2007;83:367-373.

113. Foo SS, Abbott DF, Lawrentschuk N, et al. Functional imaging of intratumoral hypoxia. Mol Imaging Biol 2004;6:291-305.

114. Aerts HJWL, Bosmans G, Baardwijk A, et al. 18F-Deoxyglucose uptake areas on PET-scan within the tumor remain stable during radiotherapy for NSCLC: a prospective study. Int J Radiat Oncol Biol Phys 2008; Jan 28: Epub ahead of print. 
SUMMARY 



\section{Introduction}

Chapter 1 contains a general introduction of the staging and treatment of non-small cell lung cancer (NSCLC). Moreover, the general role of the positron emission tomography (PET) scan in staging of NSCLC cancer is discussed and the objectives of this thesis are described.

The main questions addressed in this thesis are:

- What is the biological meaning of FDG-uptake on PET scan?

- Can FDG-PET-CT scan further improve the characterization and definition of the target volume?

- Can the treatment of NSCLC be improved by individualization of radiotherapy?

In Chapter 2 a review is given of the current role of PET(-CT) scan in radiation treatment of NSCLC. Until recently PET scan, mainly using ${ }^{18} \mathrm{~F}$-Fluoro-deoxyglucose (FDG) as a tracer was primarily used for staging purposes in NSCLC, because of its high accuracy compared with CT. Its use has in individual patients resulted in a change in stage and treatment. Nowadays PET is also increasingly used to define target volume in radiation treatment planning for NSCLC. Although both an increase as well as a decrease in target volumes has been described, most studies have shown a mean decrease of 20 to $25 \%$. Changes are mainly caused by a change in nodal target volume and the exclusion of atelectasis. Incorporation of PET seems to improve tumour coverage and spare normal tissues, which may lead to less toxicity or the possibility to escalate dose. Moreover, combining PET and CT reduces interobserver variability in target volume delineation compared with $\mathrm{CT}$ alone. However, more research is needed to assess the exact role of PET in radiation treatment of NSCLC.

\section{The biological meaning of FDG uptake in NSCLC}

The biological meaning of the uptake of ${ }^{18} \mathrm{~F}$-fluoro-2-deoxy-glucose (FDG) as visualized on PET scan is discussed in Chapter 3 and Chapter 4. The maximal uptake of FDG in the primary tumour was consistently shown to be an independent prognostic factor for survival. But the actual mechanisms by which a high FDG uptake leads to a worse prognosis are not well known. Chapter 3 describes the results of a retrospective study, in which the relation between the maximal standardized uptake value $\left(S U V_{\max }\right)$ and a proliferation-related marker (Ki-67), glucose transporters (GLUT-1 and GLUT-3) and hypoxia related markers (HIF-1a and CAIX) was investigated. One hundred and two patients, scheduled for complete resection, received a diagnostic PET scan and their surgical specimens were used for immunohistochemical staining. It was shown that the actuarial survival was worse for patients having a tumour with a high SUV $\mathrm{Vax}_{\text {. More- }}$ over, tumours with a high SUV $\max$ expressed in a higher proportion HIF-1a and GLUT-1

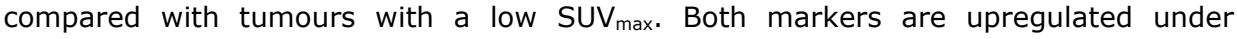
hypoxic conditions. This study provides evidence that not only in vitro, but also in vivo hypoxia is associated with an increase in FDG uptake. 
Tumours are heterogeneous for several biological characteristics; therefore it is the ultimate aim to visualize tumour characteristics by imaging. Variations in the FDG uptake within the tumour might reflect heterogeneity of ongoing pathophysiological processes. In Chapter 4 the feasibility to correlate intra-tumour heterogeneity as visualized on FDG PET with histology for NSCLC is evaluated. For this purpose we used an ex-vivo model. FDG was injected just before surgery and the surgical specimen were scanned. The procedure was feasible in all 5 patients. This method can also be applied to other tumour types and PET tracers, and can provide more insight information in the biological correlates of intra-tumour heterogeneity. Finally, this would allow for specific targeting and hence improvement of treatment outcome in NSCLC.

\section{The role of FDG-PET scan in radiotherapy of NSCLC}

Chapters 5 to 7 deal with the role of PET-CT before, during and after radiation treatment. First of all, before start of radiotherapy delineation accuracy can be improved using PET. The PET scan signal can be utilized to automatically delineate tumours. In Chapter 5 the Source-to-Background (SBR) based auto-contouring method is validated for NSCLC. The maximal tumour diameter of the SBR based auto-contour correlated strongly with the macroscopic diameter of primary tumours and was shown to be accurate for involved lymph nodes. Moreover, the influence on interobserver variability compared with manual PET-CT based gross tumour volume (GTV) delineation was investigated. The auto-contour based target volumes decreased the delineated target volumes and reduced the interobserver variability. Auto-contouring may therefore further improve the quality of target delineation in NSCLC patients.

PET-CT scan also be used to assess changes in tumour volume, tumour motion and FDG uptake during a course of fractionated radiotherapy. The results of repeated PET-CT scans and Respiration Correlated CT scans prior to treatment and in the first and second week of radiotherapy are reported in Chapter 6. A striking heterogeneity of tumour volume changes was observed at all time points. Although for the majority of patients the tumour volume only changed slightly $(<30 \%)$, in some patients $(4 / 23)$ the volume decreased $>30 \%$ and in others $(3 / 23)$ the volume increased $>30 \%$. No significant changes in average tumour motion or breathing frequencies were observed during fractionated radiotherapy. These findings underscore the need for repeated imaging during the course of radiotherapy, since an increase in volume without changing the radiation fields will lead to inadequate tumour coverage.

In Chapter 7 we investigated changes in FDG uptake during fractionated radiotherapy in relation to metabolic response after radiation treatment. Like for tumour volumes, the evolution of SUV $_{\max }$ showed a large intra-individual heterogeneity. Whereas for metabolic responders no change in $S U V_{\max }$ was observed during radiotherapy, metabolic non-responders showed an $48 \%$ increase during first week followed by a decrease in the second week. Furthermore, non-responders had a higher SUV $\max$ compared with responders at all time points investigated and showed a significantly worse overall survival compared to metabolic responders. The difference in time trends in SUV $V_{\max }$ might reflect intrinsic tumour characteristics and might finally enable adjustment of treatment. 


\section{Clinical implications: the role of individualized radiotherapy in NSCLC}

The use of PET-CT scan for target volume delineation may reduce the delineated target volume and might therefore give the possibility to escalate dose. Dose-escalation is one of the approaches to overcome the high number of local recurrences, which is one of the major problems after (chemo-)radiation in NSCLC. Therefore, for each individual patient the highest therapeutic ratio should be achieved by increasing the total tumour dose (TTD) to the limits of normal tissues; the so-called individualized maximal tolerable dose.

Chapter 8 depicts the theoretical gain in estimated tumour control probability (TCP) that can be achieved when an individualized maximal tolerable dose (MTD) prescription is used. Five treatment plans were compared; the first two employed a classic fractionation of $2 \mathrm{~Gy} /$ day (5 days/week) to a total dose of $60 \mathrm{~Gy}$ ( $\mathrm{QD}_{\text {classic }}$ ) or determined by individualized MTD dependent upon normal tissue dose constraints for lung and spinal cord $\left(\mathrm{QD}_{\text {MTD }}\right)$. The third scheme assumed a hypofractionated schedule of $2.75 \mathrm{~Gy}$ fractions ( $Q D_{\text {hypofr }}$ ), while the fourth and fifth scheme were based on hyperfrac-

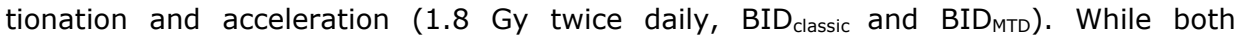
dose-escalation and hypofractionation resulted in an increase in mean estimated TCP compared with a classic fractionation to a total dose of $60 \mathrm{~Gy}$, the combination of escalation and acceleration (BID ${ }_{\text {MTD }}$ ) improved the mean estimated TCP with more than $25 \%$.

In Chapter 9 we report the first results of a prospective feasibility trial applying the MTD prescription model. All 28 patients included in this study were irradiated using an individualized prescribed total tumour dose, based on normal tissue dose constraints (mean lung dose of $19 \mathrm{~Gy}$ and maximal spinal cord dose of $54 \mathrm{~Gy}$ ) up to a maximal TTD of $79.2 \mathrm{~Gy}$ in $1.8 \mathrm{~Gy}$ fractions twice daily. The TTD was most often limited by the mean lung dose. Acute toxicity was in general mild, although 1 patient died due to pneumonitis. Concerning late toxicity, 2 patients had grade 3 toxicity. We showed that an individualized maximal tolerable dose irradiation, based on normal tissue dose constraints, is feasible and that initial results showing a median overall survival of 19.6 months are promising.

Finally, Chapter 10 contains a discussion of the results of this thesis with regard to the current standard of radiation treatment of NSCLC. Furthermore, it contains the general conclusions and future perspectives. 

SAMENVATTING 



\section{Introductie}

Hoofdstuk 1 bevat allereerst een algemene introductie met betrekking tot de stadiëring en de behandeling van longkanker. In Nederland bedraagt het aantal nieuwe patiënten met longkanker ongeveer 9000 per jaar. Longkanker is de tweede meest voorkomende kanker bij mannen en de derde bij vrouwen (www.ikcnet. $n /$ ). De prognose van longkanker is in het algemeen slecht met een 1 -jaarsoverleving van ongeveer $40 \%$ en een 5 -jaarsoverleving van $15 \%$.

Longkanker kan worden onderverdeeld in het kleincellige (20\%) en niet-kleincellige type $(80 \%)$. De behandeling van niet-kleincellige longkanker is afhankelijk van het stadium van de ziekte en de algemene conditie van de patiënt. Patiënten met een vroeg stadium van longkanker, die in een goede algemene conditie zijn, komen in aanmerking voor chirurgische resectie. Patiënten met lokaal gevorderde ziekte komen in principe in aanmerking voor een gecombineerde behandeling van radiotherapie en chemotherapie. En in het geval van metastasen kan worden gekozen voor palliatieve chemotherapie en/of ondersteunende zorg. In dit stadium kan radiotherapie een belangrijke rol spelen bij de palliatie van pijnlijke (bot)metastasen.

De positron emissie tomografie (PET) scan heeft een belangrijke rol in de diagnostiek van longkanker. De PET scan is een functionele beeldvormingstechniek, waarbij een radioactieve isotoop wordt ingebracht in de patiënt. Met behulp van een speciale camera kan de straling, uitgezonden door de radioactieve isotoop, gedetecteerd worden en kan er een afbeelding gemaakt worden van de verdeling van de isotoop in het lichaam. ${ }^{18} \mathrm{~F}$-Fluoro-deoxyglucose (FDG) is de meest gebruikte radioactieve isotoop en geeft inzicht in het glucose metabolisme.

In het eerste hoofdstuk worden eveneens de doelstellingen van dit proefschrift beschreven, waarbij een drietal vragen centraal staan:

- Wat is de biologische betekenis van opname van FDG in de longtumor op de PET scan?

- Wat is de rol van de PET scan in de radiotherapeutische behandeling van niet-kleincellige longkanker?

- Kan de behandeling van niet-kleincellige longkanker verbeterd worden door de toepassing van geïndividualiseerde radiotherapie?

In hoofdstuk 2 wordt een overzicht gegeven van de huidige rol van de PET scan in de radiotherapeutische behandeling van longkanker. De PET scan wordt veelal gebruikt voor de stadiëring van longkanker, omdat de PET scan accurater is dan de computer tomografie (CT). Op basis van de PET scan wordt in een aantal individuele gevallen een ander klinisch stadium vastgesteld, wat leidt tot een wijziging van de voorgenomen behandeling. De PET scan kan ook gebruikt worden om het doelvolume ten behoeve van de radiotherapieplanning te bepalen. Toevoeging van de PET scan informatie aan conventionele beeldvorming leidt in een aanzienlijk aantal patiënten tot een verandering van het doelvolume. Deze verandering wordt voornamelijk bepaald door een verschil in het aantal aangedane lymfeklieren op basis van de PET scan in vergelijking met de CT scan. Daarnaast biedt de PET scan de mogelijkheid om onderscheid te maken tussen tumor en atelectase (een aandoening waarbij een deel van de long geen 
lucht meer krijgt en daardoor samenvalt). Gemiddeld neemt het doelvolume door het gebruik van de PET scan 20 tot $25 \%$ af. Door het gebruik van de PET scan kan de tumor beter gevisualiseerd worden, waardoor de exacte positie van de tumor met een grotere nauwkeurigheid bepaald kan worden. Dit heeft als gevolg dat er een kleiner risico is op het geografisch missen van een deel van de tumor. Daarnaast kan de PET scan leiden tot een betere sparing van het omliggende gezonde weefsel, met als consequentie een afname van de bijwerkingen en/of de mogelijkheid tot het geven van een hogere radiotherapiedosis. Een ander voordeel van de combinatie van de PET en CT scan ten behoeve van de bepaling van het radiotherapie doelvolume, is dat de variatie van intekening tussen de verschillende artsen afneemt. Aanvullend onderzoek is echter nog nodig om de uiteindelijke rol van de PET scan in de radiotherapeutische behandeling van longkanker te bepalen.

\section{De biologische betekenis van de opname van FDG in longkanker}

De biologische betekenis van de opname van FDG, zoals deze gezien wordt op de PET scan, wordt besproken in hoofdstuk 3 en 4 . De maximale opname van FDG in de primaire tumor is een prognostische factor met betrekking tot overleving. Over het onderliggende mechanisme, waarom een hoge FDG opname leidt tot een slechte prognose, is weinig bekend. In hoofdstuk 3 worden de resultaten van een retrospectieve studie, waarin de relatie tussen de maximale opname van FDG uitgedrukt als 'standardized uptake value' $\left(S U V_{\max }\right)$ en de immuunhistochemische expressie van diverse markers beschreven. In het totaal ondergingen 102 patiënten met longkanker een PET scan voorafgaande aan een operatie. Biopten van de tumor genomen uit het longpreparaat werden gebruikt voor immunohistochemische kleuringen: een proliferatie gerelateerde marker (Ki-67), glucose transporters (GLUT-1 and GLUT-3) en hypoxie gerelateerde markers (HIF-1a and CAIX). De overleving van patiënten met een tumor met een hoge $S_{U} V_{\max }$ was beduidend slechter dan die van patiënten met een tumor met een lage $S U V_{\max }$. Bovendien toonden tumoren met een hoge $S U V_{\max }$ in grotere mate aankleuring voor de markers HIF-1a en GLUT-1 in vergelijking met tumoren met een lage $S U V_{\max }$. Beide markers komen in toenemende mate tot expressie onder hypoxische omstandigheden. Deze studie toont aan dat niet alleen in vitro, maar ook in vivo hypoxie geassocieerd is met een toename in opname van FDG.

Tumoren tonen heterogeniteit voor diverse biologische kenmerken. Het zou daarom uiterst interessant zijn als deze heterogeniteit in beeld gebracht kan worden door middel van beeldvorming. Mogelijk geeft de variatie in opname van FDG binnen een tumor, zoals deze gezien wordt op de PET scan, heterogeniteit in biologische processen binnen de tumor weer. In hoofdstuk 4 wordt een ex-vivo methode beschreven, waarin de intra-tumor heterogeniteit in FDG opname wordt gecorreleerd met onderliggende pathofysiologische processen. Hiervoor werd bij 5 patienten FDG toegediend juist voor de operatie en werd de verwijderde tumor gescand in de PET scanner. Deze methode is bruikbaar en kan eveneens toegepast worden in andere tumoren en met behulp van andere PET tracers. De ex-vivo methode kan kan meer inzicht geven in de biologische achtergrond van intra-tumor heterogeniteit zoals gevisualiseerd op de PET scan. De informatie verkregen via deze methode geeft de mogelijkheid voor specifieke, doel- 
gerichte behandeling van de verschillende subvolumina binnen een tumor met als uiteindelijke doel de verbetering van de behandelingsresultaten van niet-kleincellige longkanker.

\section{De rol van de FDG-PET scan in de radiotherapeutische behandeling van niet-kleincellige longkanker}

Hoofdstuk 5 tot en met 7 beschrijven de rol van de PET-CT scan voorafgaande aan, gedurende en na afloop van de radiotherapeutische behandeling van niet-kleincellige longkanker. Voorafgaande aan de radiotherapie kan de PET scan gemaakt worden om het doelvolume van de radiotherapie te bepalen. Het PET signaal kan gebruikt worden om de tumor automatisch in te tekenen. In hoofdstuk 5 wordt de automatische intekening van de tumor gebaseerd op de 'Source-to-Background' (SBR) methode gevalideerd voor niet-kleincellige longkanker. De maximale diameter van de primaire tumor vastgesteld door middel van de SBR methode toonde een goede correlatie met de macroscopische diameter zoals vastgesteld bij pathologisch onderzoek. Eveneens blijkt de SBR methode accuraat te zijn voor de intekening van aangedane lymfeklieren. In deze studie is ook gekeken naar de invloed van de automatische intekening op de variatie van intekening van de tumor door verschillende artsen. Als uitgangssituatie werd de intekening van 33 longtumoren door 5 artsen gebruikt. Vervolgens werd deze 5 artsen gevraagd de 33 tumoren nogmaals in te tekenen, nu met de SBR autocontour als uitgangspunt. Hierdoor nam het gemiddelde ingetekende volume af en de variatie tussen de diverse intekeningen van de artsen nam eveneens af. De SBR automatische intekenmethode verbetert de kwaliteit van de intekening bij niet-kleincellige longkanker en dient te worden gebruikt als basis voor klinische studies en in de dagelijkse praktijk.

De PET-CT scan kan eveneens gebruikt worden om veranderingen gedurende de radiotherapeutische behandelingen in beeld te brengen. In dit proefschrift worden de veranderingen in het tumor volume, de tumor beweging en de mate van opname van FDG beschreven. In hoofdstuk 6 worden de resultaten van herhaalde PET-CT scans en herhaalde ademhalingsgecorreleerde CT scans voorafgaande aan de radiotherapie en gedurende de eerste 2 weken van de radiotherapie gepresenteerd. Er werd een grote variatie in individuele tumorvolumeveranderingen gezien. Bij sommige patiënten $(4 / 23)$ neemt het tumorvolume af $(>30 \%)$, terwijl bij anderen $(3 / 23)$ het tumorvolume met meer dan $30 \%$ toeneemt. Het merendeel van de patiënten toont echter geen evidente verandering in het tumorvolume gedurende de bestralingsperiode. Deze studie toonde geen significante veranderingen in gemiddelde tumorbeweging en/of ademhalingsfrequentie gedurende de bestraling. Echter gezien het feit dat een toename in tumorvolume zonder aanpassing van de radiotherapievelden kan leiden tot een inadequate dekking van de dosis ter plaatse van de tumor, is het van wezenlijk belang om het tumorvolume gedurende de radiotherapie door middel van CT te controleren.

In hoofdstuk 7 worden de veranderingen in opname van FDG gedurende en na afloop van de radiotherapie beschreven. De veranderingen in maximale FDG opname ( $S U V_{\max }$ ) in de tumor tonen een grote variatie tussen de verschillende patiënten. De PET-CT scan 
zeventig dagen na de laastste fractie radiotherapie werd gebruikt om de mate van metabole respons te bepalen. Patiënten, die op dit tijdstip een complete of partiele respons toonde, leefden significant langer dan de zgn. metabole non-responders. Bovendien toonden deze 2 groepen patiënten verschillende patronen van SUV max $_{\text {max }}$ gedurende de bestraling. Terwijl er bij metabole responders sprake was van een stabiele SUV $\max$ gedurende de radiotherapie, toonde de groep metabole non-responders een gemiddelde toename van $48 \%$ van de $S_{U} V_{\max }$ gedurende de $1^{\mathrm{e}}$ week, gevolgd door een afname in de $2^{\mathrm{e}}$ week van de radiotherapie. Op alle onderzochte tijdstippen hadden de metabole non-responders een hogere $S_{U} V_{\max }$ als de metabole responders. Het verschil in de hoogte van de $S V_{\max }$ en het verschillende tijdsbeloop kan mogelijk veroorzaakt worden door intrinsieke tumorkarakteristieken. De veranderingen in volume dan wel opname van FDG zouden uiteindelijk gebruikt kunnen worden om de behandeling gedurende de radiotherapie aan te passen.

\section{Klinische implicaties: de rol van geïndividualiseerde radiotherapie van niet-kleincellige longkanker}

Het gebruik van de PET-CT scan voor de bepaling van het doelvolume kan leiden tot een afname van het doelvolume. Dit geeft de mogelijkheid om de radiotherapiedosis te verhogen, zonder dat daarbij een veranderd bijwerkingsprofiel van normale weefsels verwacht hoeft te worden. $\mathrm{Na}$ (chemo-)radiatie is het aantal patiënten, dat een lokaal recidief ontwikkelt echter nog erg hoog. Dosis-escalatie wordt gezien als één van de mogelijkheden om het aantal lokale recidieven te verminderen. Het is daarom van belang dat iedere individuele patiënt de hoogst mogelijke radiotherapiedosis (de zgn. totale tumor dosis, TTD) toegediend krijgt ter plaatse van de lokale tumor en de aangedane lymfeklieren. Hierbij dient de tolerantie van de gezonde weefsels wel in acht genomen te worden. Dit principe wordt de geïndividualiseerde maximaal tolerabele dosis genoemd.

In hoofdstuk 8 wordt het theoretische model van de geïndividualiseerde maximaal tolerabele dosis beschreven en de mogelijke winst in tumor controle, die hiermee behaald kan worden. In dit hoofdstuk worden 5 radiotherapieschema's met elkaar vergeleken: een conventioneel klassiek schema, een geaccelereerd radiotherapieschema (meerdere fracties per dag), een gehypofractioneerd schema (een hogere fractiedosis) en twee geïndividualiseerde schema's (een schema in éénmaal dagelijkse fracties en een geaccelereerd schema). Deze planningsstudie laat zien dat er een grote winst is te verwachten met betrekking tot de kans op tumor controle bij de toepassing van een geïndividualiseerd, geaccelereerd schema in vergelijking met alle andere radiotherapieschema's.

In hoofdstuk 9 worden de eerste resultaten beschreven van een prospectieve, klinische studie waarin het geïndividualiseerde maximaal tolerabele dosis schema werd toegepast. In totaal werden 28 patiënten geïncludeerd in de studie. De patiënten werden bestraald tot een maximaal tolerabele dosis gebaseerd op de normale weefseltolerantie, te weten een gemiddelde longdosis van $19 \mathrm{~Gy}$ en een maximale dosis op het ruggenmerg van $54 \mathrm{~Gy}$. De maximaal voorgeschreven dosis bedroeg 79,2 Gy gegeven in fracties van $1,8 \mathrm{~Gy}$ tweemaal daags. De totale bestralingsdosis werd het vaakst 
beperkt door de gemiddelde longdosis. De acute bijwerkingen waren in het algemeen mild, hoewel 1 patiënt is overleden ten gevolge van een longontsteking. Met betrekking tot de bijwerkingen op lange termijn hadden 2 patiënten een graad 3 bijwerking. Deze studie heeft aangetoond dat radiotherapie op basis van een geïndividualiseerde maximaal tolerabele dosis, waarbij rekening wordt gehouden met de normale weefseltolerantie, haalbaar is. De eerste resultaten zijn veelbelovend, en tonen een mediane overleving van 19,6 maanden en een 1 -jaars en 2 -jaars overleving van respectievelijk $57,1 \%$ en $47,1 \%$.

Ten slotte, bevat hoofdstuk 10 de discussie van de resultaten beschreven in dit proefschrift ten aanzien van de huidige standaard behandeling en de recente literatuur. In dit hoofdstuk worden eveneens de toekomstperspectieven beschreven. 

PUBLICATIONS 



\section{Publications}

J. van Loon, C. Offerman, G. Bosmans, R. Wanders, A. Dekker, J. Borger, M. Oellers, A. Dingemans, A. van Baardwijk, J. Teule, G. Snoep, M. Hochstenbag, H. Houben, P. Lambin and D. De Ruysscher. ${ }^{18}$ FDG-PET based radiation planning of mediastinal lymph nodes in limited disease small cell lung cancer changes radiotherapy fields: A planning study. Radioth Oncol (in press).

A. van Baardwijk, G. Bosmans, R.J. van Suylen, M. van Kroonenburgh, M. Hochstenbag, G. Geskes, P. Lambin and D. De Ruysscher. Correlation of intra-tumour heterogeneity on ${ }^{18}$ F-FDG PET with pathologic features in non-small cell lung cancer: a feasibility study. Radioth Oncol (in press).

M. Öllers, G. Bosmans, A. van Baardwijk, A. Dekker, P. Lambin, J. Teule, W. Thimister, A. Rhamy, D. De Ruysscher. The integration of PET-CT scans from different hospitals into radiotherapy treatment planning. Radiother Oncol 2008; Jan 18: Epub ahead of print.

A. van Baardwijk, G. Bosmans, L. Boersma, R. Wanders, A. Dekker, A.C. Dingemans, G. Bootsma, W. Geraedts, C. Pitz, J. Simons, P. Lambin and D. De Ruysscher. Individualized radical radiotherapy of non-small cell lung cancer (NSCLC) based on normal tissue dose constraints: a feasibility study. Int J Rad Oncol Biol 2008; Feb 5: Epub ahead of print.

H. Aerts, G. Bosmans, A. van Baardwijk, A. Dekker, M. Oellers, P. Lambin and D. De Ruysscher. Stability of ${ }^{18} \mathrm{~F}$-Deoxyglucose uptake areas within the tumor during radiotherapy for NSCLC: a prospective study. Int J Rad Oncol Biol 2008; Jan 28: Epub ahead of print.

A. van Baardwijk, G. Bosmans, S.M. Bentzen, L. Boersma, A. Dekker, R. Wanders, B.G. Wouters, P. Lambin and D. De Ruysscher. Radiation dose prescription for nonsmall cell lung cancer based on normal tissue dose constraints: an in silico clinical trial. Int J Rad Oncol Biol 2008; Feb 5: Epub ahead of print.

G. Bosmans, A. van Baardwijk, A. Dekker, M. Öllers, S. Wanders, L. Boersma, $P$. Lambin and D. De Ruysscher. Time trends in nodal volumes and motion during radiotherapy for patients with Stage III Non-Small-Cell Lung Cancer. Int J Radiat Oncol Biol 2007; Nov 7:Epub ahead of print.

A. van Baardwijk, D. De Ruysscher, G. Lammering, B. Wouters and P. Lambin. De toekomst van radiotherapie gezien vanuit het oogpunt van de radiobiologie. Nederlands Tijdschrift voor Oncologie 2007:4(7):292-8.

J. Orban de Xivry, G. Janssens, G. Bosmans, M. De Craene, A. Dekker, J. Buijsen, A. van Baardwijk, D. De Ruysscher, B. Macq and Ph. Lambin. Tumour delineation and cumulative dose computation in radiotherapy based on deformable registration of Respiratory Correlated CT images of lung cancer patients. Radioth Oncol 2007;85(2):232-8. 
J. Stroom, J. Blaauwgeers, A. van Baardwijk, L. Boersma, J. Lebesque, J. Theuws, R. van Suylen, H. Klomp, K. Liesker, R. van Pel, C. Siedschlag and K. Gilhuijs. The feasibility of pathology-correlated lung imaging for accureate target defenition of lung tumors. Int J Rad Oncol Phys 2007;69(1):267-75.

A. van Baardwijk, C. Dooms, R. van Suylen, E. Verbeken, M. Hochstenbag, C. DehingOberije, D. Rupa, S. Pastorekova, S. Stroobants, U. Buell, P. Lambin, J. Vansteenkiste and D. De Ruysscher. The maximum uptake of ${ }^{18} \mathrm{~F}$-deoxyglucose on PET scan correlates with survival, Hypoxia Inducible Factor-1a and GLUT-1 in Non-Small Cell Lung Cancer. Eur J Cancer 2007;43(9):1392-8.

A. van Baardwijk, G. Bosmans, L. Boersma, J. Buijsen, S. Wanders, M. Hochstenbag, R. van Suylen, A. Dekker, C. Dehing-Oberije, R. Houben, S.M. Bentzen, M. van Kroonenburgh, P. Lambin and D. De Ruysscher. PET-CT scan based auto-contouring in non-small cell lung cancer correlates with pathology and reduces interobserver variability in the delineation of the primary tumor and involved nodal volumes. Int $J$ Radiat Oncol Biol Phys 2007;68(3):771-8.

A. van Baardwijk, G. Bosmans, A. Dekker, M. van Kroonenburgh, L. Boersma, S. Wanders, M. Öllers, R. Houben, A. Minken, P. Lambin and D. De Ruysscher. Time trends in the maximal uptake of FDG on PET scan during thoracic radiotherapy in relation to metabolic response. A prospective study in locally advanced non-small cell lung cancer (NSCLC) patients. Radiother Oncol 2007;82(2):145-52.

G. Bosmans, A. van Baardwijk, A. Dekker, M. Öllers, L. Boersma, A. Minken, P. Lambin and $D$. De Ruysscher. Intra-patient variability of tumor volume and tumor motion during conventionally fractionated radiotherapy for locally advanced non-small cell lung cancer. A Prospective Clinical Study. Int J Rad Oncol Biol Phys 2006;66(3):748-53.

D. De Ruysscher, R. Bremer, F. Koppe, S. Wanders, E. van Haren, M. Hochstenbag , W. Geeraedts, C. Pitz, J. Simons, G. ten Velde, J. Dohmen, G. Snoep, L. Boersma, T. Verschueren, A. van Baardwijk, C. Dehing, M. Pijls, A. Minken and P. Lambin. Omission of elective node irradiation on basis of CT-scans in patients with limited disease small cell lung cancer: a phase II trial. Radiother Oncol 2006;80(3):307-12.

A. van Baardwijk, B. G. Baumert, G. Bosmans, M. van Kroonenburgh, S. Stroobants, V. Gregoire, P. Lambin and D. De Ruysscher. The current status of FDG-PET in tumour volume definition in radiotherapy treatment planning. Canc Treat Rev 2006;32(4):24560.

A. van Baardwijk, J. de Jong, A. Arens, P. Thimister, G. Verseput, B. Kramer and P. Lambin. False-positive FDG-PET scan due to brown tumours. Eur J Nucl Med Mol Imaging 2006;33(3):393-4.

Q. de Lussanet, W. Backes, A. Griffioen, A. Padhani, C. Baeten, A. van Baardwijk, P. Lambin, G. Beets, J. van Engelshoven and R. Beets-Tan. Dynamic contrast-enhanced magnetic resonance imaging of radiation therapy-induced microcirculation changes in rectal cancer. Int J Radiat Oncol Biol Phys 2005;63(5):1309-15. 
D. De Ruysscher, S. Wanders, A. Minken, A. Lumens, J. Schiffelers, C. Stultiens, S. Halders, L. Boersma, A. van Baardwijk, T. Verschueren, M. Hochstenbag, G. Snoep, B. Wouters, S. Nijsten, S.M. Bentzen, M. van Kroonenburgh, M. Ollers and P. Lambin. Effects of radiotherapy planning with a dedicated combined PET-CT-simulator of patients with non-small cell lung cancer on dose limiting normal tissues and radiation dose-escalation: a planning study. Radiother Oncol 2005;77(1):5-10.

D. De Ruysscher, S. Wanders, E. van Haren, M. Hochstenbag, W. Geeraedts, I. Utama, J. Simons, J. Dohmen, A. Rhami, U. Buell, P. Thimister, G. Snoep, L. Boersma, T. Verschueren, A. van Baardwijk, A. Minken, S.M. Bentzen and P. Lambin. Selective mediastinal node irradiation based on FDG-PET scan data in patients with non-smallcell lung cancer: a prospective clinical study. Int $J$ Radiat Oncol Biol Phys 2005;62(4):988-94.

A. Grubben, A. van Baardwijk, D. Broering and A. Hoofwijk. Pathophysiology and clinical significance of the abdominal compartment syndrome. Zentralbl Chir 2001;126:605-9.

A. van Baardwijk, M. Verhaegh, G. Krekels, A. Vermeulen and H. Neumann. Mohs' method of micrographic surgery as treatment for recurrent basal cell carcinoma. Ned Tijdschr Geneeskd 1997;141:524-9. 

DANKWOORD 

$\mathrm{Nu}$ het wetenschappelijke deel geschreven is, hoop ik weer meer tijd te krijgen voor mijn hobby's, waaronder koken. Al lijkt het doen van onderzoek en het schrijven van een proefschrift wel op het bereiden van een menu. Ik wil $u$ in dit dankwoord dan ook graag meenemen in de bereiding van mijn menu.

De bereiding van een menu begint met het bedenken ervan. Gelukkig werd het menu mede opgesteld door Prof. Lambin, de promotor en Dr. De Ruysscher, de copromotor. Beste Philippe, ik ben je zeer erkentelijk voor de geboden kansen en het vertrouwen: de opleiding, de mogelijkheid om 3 jaar full-time onderzoek te doen en je bijdrage aan de artikelen en presentaties. Beste Dirk, mede door jouw begeleiding, sturend en steeds kritisch, is dit proefschrift tot stand gekomen. Je grenzenloze enthousiasme, de tijd die je nam om te discussiëren en je uitgebreide ervaring in het doen van onderzoek en het schrijven van wetenschappelijke artikelen hebben mede tot dit resultaat geleid.

Daarnaast is net zoals bij koken een goede voorbereiding van essentieel belang. Het begint met een goede basis: goede omstandigheden en de juiste ingrediënten.

Het is erg fijn om in een goede keuken te mogen koken. Zo is er het fantastische longteam, waar ik deel vanuit mocht maken. Beste Rinus, bedankt voor de vele intekeningen en je klinische bijdrage. Beste Liesbeth, bedankt voor de vele leerzame discussies en je kritische beoordelingen van de manuscripten. Het is soms niet leuk om te zien dat je eigen recept wordt aangepast, maar als vervolgens het eindresultaat dusdanig verbeterd, geeft dat zeker een goed gevoel.

Geert het was fijn dat we samen in dezelfde pan konden roeren. Jouw fysische toevoegingen hebben mijn medische recepten zeker verrijkt. Onze gezamenlijke inspanningen hebben dan ook geleid tot een mooi zeven gangen menu. Hoewel Geert de grootste fysische bijdrage heeft geleverd, wil ik zeker ook een aantal andere fysici bedanken. André Minken, jammer dat je je nu toelegt op de Overijsselse keuken, maar bedankt voor je bijdrage aan de eerste PET-CT recepten. André Dekker, bedankt voor je kritische houding ten aanzien van de studieopzet, de analyse en de manuscripten. Hugo, bedankt dat je samen met mij in de 'heterogene brei' wilde roeren. Michel, onze 'PET fysicus', bedankt voor je ondersteuning en je inspanningen voor de autocontouring. Wouter, bedankt dat je de recepten in Matlab hebt willen uitwerken.

Ik heb voor het bereiden van dit menu ook gebruik mogen maken van de faciliteiten van aangrenzende keukens. De longartsen en longchirurgen van het azM, Atrium Medisch Centrum, Maasland Ziekenhuis Sittard, Laurentius Ziekenhuis Roermond en Sint Jansgasthuis Weert, wil ik bedanken voor hun medewerking aan de verschillende studies. In het bijzonder wil ik Monique Hochstenbag en Anne Marie Dingemans bedanken voor hun bijdragen. Ook de afdeling pathologie heeft een groot aandeel gehad in de bereiding van het menu. Anique Janssen bedankt voor de introductie in immunohistochemie, en de groep arts-assistenten voor het uitsnijden van de vele longpreparaten. Beste Robert-Jan, bedankt voor het beoordelen van de vele coupes. Het was altijd leerzaam en bovendien gezellig om menig recept met je te delen en over begeleidende wijnen te discussiëren. 
Lekker eten staat of valt natuurlijk ook bij het gebruik van goede, bij voorkeur verse ingrediënten. Het zal u niet zijn ontgaan dat het belangrijkste ingrediënt van dit menu de PET-CT scan is. Het gebruik van PET beelden voor de radiotherapie planning vereist een goede samenwerking tussen de afdeling Nucleaire Geneeskunde en de afdeling Radiotherapie. Ik wil dan ook de afdeling Nucleaire Geneeskunde van het azM bedanken voor hun ondersteuning. Beste Marinus, bedankt voor je bijdrage aan de diverse protocollen en je aanvullingen op de manuscripten vanuit een nucleair oogpunt.

Ingrediënten, die hier niet direct leverbaar waren werden in het buitenland ingeslagen. Beste Christophe Dooms, Johan Vansteenkiste, Erik Verbeken en Sigrid Stroobants (Leuven) bedankt voor de fijne samenwerking. Beste Søren Bentzen, bedankt voor je bijdrage aan de auto-contouring en de in silico studie.

De inkoop van de beste ingrediënten is alleen mogelijk met een goed budget. Ik wil het onderzoeksinstituut GROW (UM) en Biocare (EC FP6) dan ook bedanken voor de geboden mogelijkheden: het doen van onderzoek, maar ook de kans om de resultaten in binnen- en buitenland te presenteren.

En natuurlijk wil ik iedereen bedanken, die direct of indirect heeft bijgedragen aan een van de gangen van dit menu. Allereerst de patiënten, die bereid waren herhaalde PETCT scans te ondergaan. Daarnaast de co-auteurs en mijn directe collega's: de radiotherapeut-oncologen, de laboranten, de (secretariële) ondersteuning en niet te vergeten de groep arts-assistenten. Als onderzoekster mocht ik toch een kamer met ze delen en er was altijd een kop koffie en een luisterend oor. Cary en Ruud wil ik bedanken voor de statistische ondersteuning, Bianca voor de bewerking van de foto's en Rianne voor de secretariële ondersteuning van de promotie.

Zoals ook met koken is de receptuur van dit proefschrift in de loop van de tijd regelmatig aangepast. In de beginfase was er nog de experimentele keuken: Kim, Ludwig en Jan bedankt voor jullie hulp bij de 'in-vitro' recepten. Goede ideeën en suggesties van collega's hebben uiteindelijk geleid tot verdere verbeteringen van het menu, waardoor de gangen beter op elkaar zijn afgestemd.

Lekker eten hoort natuurlijk begeleid te worden door een goed glas wijn. De wijn moet ten eerste op dronk en in evenwicht met het eten zijn. Daarnaast heb je natuurlijk nog de persoonlijke voorkeur: de één prefereert een Rioja, terwijl de ander de voorkeur geeft aan een Bourgogne wijn. Ik heb ook geleerd dat het belangrijk is om een fles wijn soms even te laten liggen om deze verder op dronk te laten komen. En dat geldt ook voor sommige ideeën in dit proefschrift: deze hebben even op het vat moeten rijpen voordat ze tot uitvoering zijn gebracht.

Verder wil ik de beoordelingscommisie graag bedanken voor het voorproeven van dit menu: Prof. van Engelshoven, Prof. Grosu, Prof. Teule en Dr. ten Velde bedankt dat u de tijd heeft gevonden om dit menu te beoordelen en van waardevolle opmerkingen te voorzien. 
$\mathrm{Nu}$ het menu bijna klaar is, dient het te worden opgediend en hierbij hoort natuurlijk een mooie opmaak van de gerechten. Patricia, bedankt voor het ontwerp en de professionele opmaak van de omslag.

Het belangrijkste van een menu is echter het genieten ervan samen met familie en vrienden. Ik wil mijn ouders bedanken voor hun steun en hun vertrouwen in mij. Mede dankzij jullie voorbeeld om door te zetten, heb ik dit bereikt. Daarnaast wil ook alle familie en vrienden bedanken, waarmee ik regelmatig gezellig heb mogen tafelen. Tijdens deze etentjes kwam geregeld mijn onderzoek ter sprake: bedankt voor jullie interesse en enthousiaste reacties. In het bijzonder wil ik mijn paranymfen Saskia en Jeroen bedanken.

Tenslotte lieve Marcel bedankt dat je de bereiding en het opdienen van dit menu samen met mij hebt willen delen. Bedankt voor je steun, je begrip en je liefde.

Ik zou u nu graag allen willen uitnodigen om samen met mij aan tafel te gaan en te gaan genieten van dit menu. 

CURRICULUM VITAE 



\section{Curriculum Vitae}

Angela van Baardwijk werd geboren op 23 september 1971 in Waalwijk en groeide op in Drunen. In 1989 voltooide zij haar VWO diploma aan het Dr. Mollercollege in Waalwijk en aansluitend startte zij met de studie Geneeskunde aan de Universiteit Maastricht. In september 1996 behaalde zij het artsexamen en werkte ruim 2 jaar als assistent geneeskunde niet in opleiding Heelkunde in het Maasland Ziekenhuis in Sittard. In februari 1999 werd gestart met de opleiding Heelkunde in het academisch ziekenhuis Maastricht (opleiders: Prof. G. Kootstra en Prof. M. Jacobs). In april 2001 verwisselde zij op eigen initiatief de opleiding Heelkunde voor de opleiding tot radiotherapeut-oncoloog in het Radiotherapeutisch Instituut Limburg (de huidige MAASTRO clinic) met als opleiders Dr. J. Jager en Prof. P. Lambin. Vanaf 2004 werd de opleiding gedurende 3 jaar onderbroken voor het promotie onderzoek, zoals beschreven in dit proefschrift. Dit onderzoek vond plaats binnen het onderzoeksinstituut 'Growth and Development' van de Universiteit Maastricht onder directe begeleiding van Dr. D. De Ruysscher en Prof. P. Lambin als promotor. In juli 2007 hervatte zij de opleiding Radiotherapie binnen MAASTRO clinic, die zij in oktober 2008 verwacht af te ronden. 


